\title{
New ideas for handling of loop and angular integrals in D-dimensions in QCD
}

\author{
Valery E. Lyubovitskij, ${ }^{a, b, c, 1}$ Fabian Wunder $^{a}$ and Alexey S. Zhevlakov ${ }^{d, c, e}$ \\ ${ }^{a}$ Institut für Theoretische Physik, Universität Tübingen, \\ Kepler Center for Astro and Particle Physics, \\ Auf der Morgenstelle 14, D-72076 Tübingen, Germany \\ ${ }^{b}$ Departamento de Física y Centro Científico Tecnológico de Valparaíso-CCTVal, \\ Universidad Técnica Federico Santa María, \\ Casilla 110-V, Valparaíso, Chile \\ ${ }^{c}$ Department of Physics, Tomsk State University, \\ 634050 Tomsk, Russia \\ ${ }^{d}$ Bogoliubov Laboratory of Theoretical Physics, Joint Institute for Nuclear Research, \\ 141980 Dubna, Russia \\ ${ }^{e}$ Matrosov Institute for System Dynamics and Control Theory SB RAS, \\ Lermontov str. 134, 664033 Irkutsk, Russia \\ E-mail: valeri.lyubovitskij@uni-tuebingen.de, \\ fabian.wunder@student.uni-tuebingen.de, zhevlakov@theor.jinr.ru
}

ABSTRACT: We discuss new ideas for consideration of loop diagrams and angular integrals in $D$-dimensions in QCD. In case of loop diagrams, we propose the covariant formalism of expansion of tensorial loop integrals into the orthogonal basis of linear combinations of external momenta. It gives a very simple representation for the final results and is more convenient for calculations on computer algebra systems. In case of angular integrals we demonstrate how to simplify the integration of differential cross sections over polar angles. Also we derive the recursion relations, which allow to reduce all occurring angular integrals to a short set of basic scalar integrals. All order $\varepsilon$-expansion is given for all angular integrals with up to two denominators based on the expansion of the basic integrals and using recursion relations. A geometric picture for partial fractioning is developed which provides a new rotational invariant algorithm to reduce the number of denominators.

Keywords: Perturbative QCD, Gauge Symmetry, Scattering Amplitudes

ARXIV EPRINT: 2102.08943

\footnotetext{
${ }^{1}$ Corresponding author.
} 


\section{Contents}

1 Introduction 1

2 New formalism for reduction of tensor loop integrals $\quad 7$

2.1 Bubble integrals $\quad 9$

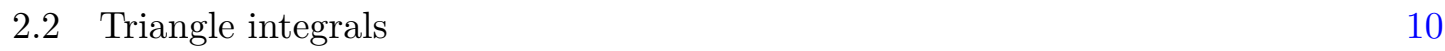

$\begin{array}{lll}2.3 & \text { Box integrals } & 14\end{array}$

3 Angular integrals in $D$ dimensions $\quad 18$

3.1 Generalized two particle phase space in $D$ dimensions 18

3.2 Analytic evaluation of angular integrals in $D$ dimension 20

$\begin{array}{lll}3.2 .1 & \text { Integral without denominator } & 21\end{array}$

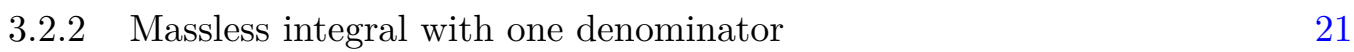

3.2.3 Massive integral with one denominator $\quad 22$

3.2.4 Massless integral with two denominators 24

$\begin{array}{ll}3.2 .5 & \text { Single massive integral with two denominators }\end{array}$

3.2.6 Double massive integral with two denominators 28

$\begin{array}{lll}3.3 & \text { New properties of angular integrals } & 29\end{array}$

3.3.1 Hypergeometric representation of angular integrals 29

$\begin{array}{lll}\text { 3.3.2 Partial differential identities } & 32\end{array}$

$\begin{array}{lll}\text { 3.3.3 Partial integration identities } & 37\end{array}$

$\begin{array}{lll}3.3 .4 & \text { Recursion relations } & 38\end{array}$

3.4 All order $\varepsilon$-expansion of angular integrals 43

3.4.1 Massive integral with one denominator 43

3.4.2 Massless integral with two denominators $\quad 45$

3.4.3 Single massive integral with two denominators 46

3.4.4 Double massive integral with two denominators 48

$\begin{array}{lll}3.5 & \text { Neerven integrals } & 49\end{array}$

3.5.1 Connection between Neerven and Somogyi parametrization 49

3.5.2 On massless integral in the Neerven parametrization $\quad 51$

3.5.3 On massive integral in the Neerven parametrization 51

3.5.4 Recursive determination of Mirkes type III integrals 52

4 Conclusions $\quad 54$

$\begin{array}{ll}\text { A Triangle integrals } & \mathbf{5 6}\end{array}$

$\begin{array}{ll}\text { A.1 Scalar and vector triangle integrals } & 56\end{array}$

A.2 Tensor triangle integrals 64 
B Box integrals $\quad 75$

B.1 Scalar box integral $\quad 75$

B.2 Vector box integrals $\quad 81$

B.3 Tensor box integrals 83

$\begin{array}{lll}\text { C Spherical integration in dimensional regularization } & 90\end{array}$

D Partial fraction decomposition of linear propagators $\quad 92$

D.1 Two-point splitting lemma $\quad 92$

D.2 Two-mass splitting and two-point partial fractioning 94

D.3 Three-point partial fractioning and general denominator reduction $\quad 95$

$\begin{array}{lll}\text { D.4 Propagators to negative integer powers } & 98\end{array}$

$\begin{array}{ll}\text { D.5 Connection to physical propagators } & 99\end{array}$

D.6 Example: Drell-Yan double real corrections kinematics 100

E Consistency of the hypergeometric representation of angular integrals 103

$\begin{array}{lll}\text { E.1 Double massive integral in four dimensions } & 104\end{array}$

$\begin{array}{lll}\text { E.2 Single massive integral } & 104\end{array}$

$\begin{array}{lll}\text { E.3 Massless integral } & 106\end{array}$

$\begin{array}{ll}\text { F Double Nielsen polylogarithms } & 107\end{array}$

$\begin{array}{lll}\text { F.1 Basic properties of double Nielsen polylogarithms } & 107\end{array}$

$\begin{array}{lll}\text { F.2 Relation of Goncharov and Nielsen polylogarithms } & 107\end{array}$

$\begin{array}{lll}\text { F.3 Double Nielsen polylogarithms in terms of multiple polylogarithms } & 108\end{array}$

G Tables of angular integrals 109

$\begin{array}{ll}\text { G.1 Massless integral with one denominator } & 109\end{array}$

$\begin{array}{lll}\text { G.2 Massive integral with one denominator } & 110\end{array}$

$\begin{array}{lll}\text { G.3 Massless integral with two denominators } & 110\end{array}$

$\begin{array}{ll}\text { G.4 Single massive integral with two denominators } & 113\end{array}$

$\begin{array}{lll}\text { G.5 Double massive integral with two denominators } & 119\end{array}$

$\begin{array}{lll}\text { G.6 } & \text { Mirkes type III integrals } & 119\end{array}$ 


\section{Introduction}

Evaluation of loop and angular integrals is one of the basic tasks in quantum field theory. During last five decades huge progress has been achieved in analytical handling of loop integrals in QCD (see, e.g., refs. [1-41]). While many different methods for evaluating loop diagrams have been developed, they mainly use the following techniques:

- Reduction of tensor structure of loop diagrams using the Passarino-Veltman (PV) method [3] based on Lorentz covariance of matrix elements and fractioning of the denominators in loop integrals. For extensions of the alternative PV methods up to six external legs see ref. [26];

- Reduction of numerators containing virtual momenta by means of derivatives acting on linear combinations of external momenta [11, 18, 19, 24, 29, 30, 33];

- Reduction of tensor to scalar integrals with shifted dimension [11], which further reduce to the loop integrals in generic dimension $[18,19]$;

- using helicity methods in evaluating loop integrals $[8,9]$;

- Method based on integration by parts (IBP) reduction is widely used for handling two and higher loops integrals [21];

- Methods based on decomposition of Lorentz vectors in a parallel and orthogonal space in the context of one-loop and multi-loop integrand reduction, and generalized unitarity $[13,14,23,27,28,31,32,39]$;

- Development of analytical and numerical methods for $\varepsilon$-expansion of loop integrals (see, e.g., refs. [34, 41]).

In particular, the PV method [3] is based on expanding integrals, which contain the loop momentum in the numerator, in terms of a set of scalar functions (form factors) multiplying a basis of tensors constructed from external momenta. The form factors can be expressed by the readily calculated scalar bubble, triangle and box one-loop integrals. The scalar form factors of tensorial loop integrals are obtained algebraically by solving a system of linear equations. This leads to the introduction of the Gram determinant composed of scalar products of external momenta. As correctly stressed in ref. [19] the usage of the PV method is best suited for diagrams with four or less external legs, otherwise the tensor structure of the diagrams with multi-legs becomes more complicated due to solving systems of many algebraic equations and due to vanishing Gram determinants at some kinematic cases.

Success of the PV method [3] stimulated further development of the idea to use the combinations of external momenta for the construction of basis for the expansion of tensorial loop integrals. In particular, the decomposition of Lorentz vectors in a parallel and orthogonal space has already been extensively used in the context of one-loop and multi-loop integrand reduction, generalized unitarity and recent developments in the decomposition of amplitudes into tensors and form factors (see, e.g., discussion in refs. [27, 31, 39]). In ref. [26] the PV method was extended using alternative techniques up to six external legs. 
In the present paper we introduce the following improvement of the PV method. Instead of expanding loop integrals in the trivial basis of occurring external momenta we perform the expansion in the basis of orthogonal linear combinations of the external momenta. It allows us to exclude the stage of algebraically solving a system of equations in order to pin down the scalar functions in which we expand tensor loop diagrams. Instead we derive these scalar functions straightforwardly using fractioning relations involving inverse denominators in loop integrals.

We consider only loop integrals without referring to specific type of the particles propagating in the loop. The proposed formalism can be adapted to full amplitudes.

Our method is very useful for a convenient analytic treatment of processes with massless particles, e.g., Drell-Yan (DY) and SIDIS processes, prompt photon and heavy flavor production, etc. However, it is important to notice that the proposed method can be generalized to arbitrary number of legs in one-loop diagrams and to massive particles.

While for many cutting-edge perturbative studies, like inclusive DY processes at $N^{3} \mathrm{LO}$ (see, e.g., refs. [90, 91]), multi-loop techniques are key, we would like to stress that studies where a refinement of one-loop techniques can provide considerable improvement are still an active area of research.

For understanding angular distributions, spin effects and for access to the parton distributions (PDFs and TMDs), improving on the existing tools in producing analytical expressions as simply and economically as possible is beneficial. Besides the importance of analytical methods for phenomenological study of QCD processes in the fixed target regime (DY, SIDIS, etc.), handy analytical expressions are also important for performing resummation of large logarithms, verifying of different identities (like Lam-Tung relation) involving hadronic structure functions, behavior of the hadronic amplitudes at different kinematical limits and their expansions in small parameters (like small transverse momentum of photon, etc.). See, e.g., discussion in refs. [42, 43].

In the second part of our paper we discuss new ideas concerning angular integration in QCD [44-50]. We extend the known closed results for massless and single massive two denominator integrals in $D$ dimensions to the double massive case. All order $\varepsilon$-expansion is given for all angular integrals with up to two denominators. A geometric picture for partial fractioning is developed which provides a new rotational invariant algorithm to reduce the number of denominators.

For the calculation of real emission corrections in higher order perturbative calculations the phase space integration (PSI) constitutes the most challenging part. When massless external particles are involved, the integrals are singular, thus numerical integration is not feasible. To regularize the divergent integrals, analytic integration methods are necessary. A prominent class of divergences are those of collinear type. They appear if two external massless particles propagate in the same direction making their scalar product vanish. These singularities appear in the angular PSI. Henceforth, analytic methods for their calculation are essential.

Since its introduction, the dimensional regularization (DR) [1] by t'Hooft and Veltman is the preferred regularization method in gauge theories. In particular, it gives the straightforward and consistent recipe to perform QCD calculations in $D$ dimensions and take the physical limit $D \rightarrow 4$ only after the cancellation of all divergences. Throughout the paper 
we choose the most common definition of $D=4-2 \varepsilon$. Note that some of the references use $D=4 \pm \varepsilon$ instead. The collinear divergences appearing in angular PSI manifest themselves as poles in the Laurent expansion about $\varepsilon=0$.

Angular integrals appear in the calculation of the two, three and four particle PSIs and thus appear in a plethora of perturbative calculations. QCD examples include processes such as Drell-Yan [49, 55-59], deep inelastic scattering (DIS) [60], semi-inclusive DIS (SIDIS) [61, 62], prompt photon production [63], hadron-hadron scattering [64], and heavy quark production $[65,66]$.

In the literature those angular integrals were mostly considered in terms of the integrals, which we call in our paper the Neerven integrals $[44,65]$ :

$$
I_{D}^{j, l}=\int \mathrm{d} \Omega_{k_{1} k_{2}} \frac{1}{\left(a+b \cos \theta_{1}\right)^{j}\left(A+B \cos \theta_{1}+C \sin \theta_{1} \cos \theta_{2}\right)^{l}},
$$

with

$$
\int \mathrm{d} \Omega_{k_{1} k_{2}}=\int_{0}^{\pi} \mathrm{d} \theta_{1} \sin ^{D-3} \theta_{1} \int_{0}^{\pi} \mathrm{d} \theta_{2} \sin ^{D-4} \theta_{2} .
$$

They are divided in four classes depending on whether the conditions $a^{2}=b^{2}$ and $A^{2}=$ $B^{2}+C^{2}$ hold. We will call $I_{D}^{(j, l)}$ massless, if both equations are satisfied and single massive, if one of them holds and else double massive. The latter are finite for $D=4$, the other two involve singularities.

We will start our discussion with an overview of how those integral were used in the older literature. From their introduction in the early 80s onwards they were an important ingredient for calculations at the NNLO level. After providing context on the development of the knowledge about angular integrals, we discuss how the use of angular integrals compares to the method of reversed unitarity, which is in frequent use today. This will come along with a review of modern application of angular integrals, which demonstrates that, thus known for four decades in QCD calculations, angular integrals are still in use today and a more systematic study of these was long overdue.

First application of DR for treatment of divergences in the angular PSIs was performed by Ellis et al. in ref. [64]. In particular, angular integrals $\int \mathrm{d} \Omega_{k_{1} k_{2}}$ as part of the phase space $\mathrm{dPS}_{3}$ were considered and the massless integrals $I_{D}^{(1,1)}$ were introduced, to which all appearing PSIs could be reduced "by suitable rotation and partial fractioning". In a similar fashion ref. [60] treated the three particle PSI. In ref. [60] partial fractioning was introduced using identities involving the Mandelstam variables in order to reduce the PSIs to the massless $I_{D}^{(1,1)}$ and $I_{D}^{(1,0)}$. Ref. [45] extended the calculation of angular integrals to the single massive $I_{D}^{(1,1)}$, while a list of the double massive integrals $I_{4}^{(j, l)}$ for $-2 \leq j, l \leq 2$ restricted to the four-dimensional case has been derived in ref. [55].

In this vein, major achievement was Neerven's analytic calculation of the massless integrals $I_{D}^{(j, l)}$ in ref. [44]. By calculating them as the discontinuity of a box graph and employing the optical theorem he heavily influenced the approach towards PSIs. For a long time this constituted the only published detailed calculation of angular integrals. Other papers were heavily based on the results of ref. [44]. The most commonly used reference 
for the angular integrals for the last three decades is [65]. Ref. [65] gave the angular integral in the form of eq. (1.1) using the set of parameters $\{a, b, A, B, C\}$ and produced a comprehensive, often cited list of angular integrals. The double massive integral $I_{4}^{(j, l)}$ was given for the case $|j|,|l| \leq 2$. The divergent integrals were expanded up to order $\varepsilon$, the massless integral was given for $j=1,-2 \leq l \leq 1$, and the single massive for $l=1$, $j=-2,-1,1,2$ and $l=2, j=0,1,2$. The single massive integrals were given in two groups without making the symmetry between $a^{2} \neq b^{2}$ and $A^{2} \neq B^{2}+C^{2}$ explicit. The list of the integrals was based on the calculation method put forward in refs. [44, 45, 55]. A similar list was compiled in ref. [46]. Some early use of those lists can be found in ref. [56]. Ref. [56] mentioned the massless and single massive integrals $I_{D}^{(j, l)}$ and stated that the single massive integral "cannot be written in as elegant a form as" the massless integral, hence "brute force" methods were used. Ref. [56] derived an early version of two-point partial fractioning discussed in more details in appendix D of the present paper. Ref. [57] considered the angular integration as part of the three-particle phase space. Again eq. (1.1) appeared for the massless case. Note that only two-point partial fractioning was mentioned in ref. [56] and there was no three-point partial fractioning (see appendix D). So seven integrals from the list of ref. [56], including those containing the massive propagator, were said to be integrated by "brute force methods". In the follow-up paper [58] the Neerven integral came with a reference to the 1989 paper [65]. The single massive integral was described as "very cumbersome". About the double massive case they write "fortunately [the double massive integral] can be avoided by choosing an appropriate frame." As a benchmark, their partial fractioning algorithm led to 217 different 3-body PSI.

Later, in ref. [49] the Neerven integral was considered as part of a four-body phase space by Mirkes. The three classes of angular integrals he lists correspond to massless and single-massive integrals. Some of the single massive integrals were expanded up to order $\varepsilon$. The recursion given for his class III integrals without reference fail for $\varepsilon \neq 0$. We correct this shortcoming in section 3.5.4. The partial fractioning was described as "very involved". In ref. [63] the Neerven integral together with "extensive use of relations between Mandelstam variables" were employed for partial fractioning to calculate the 3body phase space of prompt photon production. Additional phase space factors were accommodated for by dimensional shift $\varepsilon \rightarrow \varepsilon-1$. Such applications motivate to publish not only $\varepsilon$-expansions but also general results permitting for expansion about other values of $\varepsilon$. We consider algebraic identities to perform dimensional shifts in section 3.3.2. Further consideration of the Neerven type integrals in the context of the three particle phase space has been considered in ref. [66]. The "extensive partial fractioning" based on Mandelstam identities "though computerized [...] has the disadvantage of often yielding unnecessarily complicated expressions". It leads to massless, single-massive and double-massive Neerven integrals. The symmetry between the two classes of single-massive integrals was recognized and relations between the double-massive integrals via differentiating with respect to $a$ and $A$ were given (compare 3.5.3).

In the decade following Neervens paper, there was not much improvement on the treatment of angular integrals. In 2001 Anastasiou and Melnikov [50] proposed a new method called reverse unitarity for calculations of phase space integrals. Expressing these integrals 
in terms of loop integrals made them accessible through the sophisticated mathematical methods developed for handling of loop integrals such as integration-by-parts relations, differential equations, etc. This approach computes cut diagrams as solutions of differential equations $[53,54]$. Technically the latter are needed to be augmented with boundary conditions coming from a separate calculation. Analytic results are obtained in terms of harmonic polylogarithms order by order in $\varepsilon$. During the last twenty years these methods received an evolution $[51,52]$. The method of reverse unitarity has certain advantages. It is applicable to rather complicated PSIs since it takes full advantage of the knowledge about loop integrals. Since every delta function in the phase space translates to additional propagators, the technique is especially suited for the treatment of inclusive PSIs. Limitations are induced by the development of technical methods for calculation of loop integrals. Difficulties in these approaches grow with the inclusion of more particles in the finals state, multiple scales and massive particles.

The success of methods based on reversed unitarity provides an explanation of the scarcity of improvements regarding angular integrals. However, the direct approach towards angular integrals, rather than treating all PSIs by reversed unitarity still has its merits for certain applications. It is conceptually simple, since it makes straightforward use of their properties rather than taking a detour to loop integrals. One might compare the calculation of the massless Neerven integral in ref. [44] to ours in section 3.2.4 as an example. By considering a specific class of PSIs, in the present paper angular integrals with two denominators, analytic results can be obtained in closed form and expanded to all orders in $\varepsilon$. Furthermore, recursion relations can explicitly be solved. This yields a completely solved building block which can be plugged into calculations, without the need to run an IBP reduction and to solve differential equations order by order. The expansion presented in section 3.4 has the benefit to be compatible with commonly used methods since the result is expressed in terms of multiple polylogarithms. Of course, employing the angular integral discussed here is only applicable for observables with phase space integrals of appropriate form and is not simply generalizable for other PSIs. However, the ideas presented should also be of value for related PSIs like angular integrals with 3 and more denominators. Such integrals appear, e.g., as master integrals in the reversed unitarity method (see ref. [72]).

After introducing the angular integral in its historical context, we will look at modern developments and usage in the following. In 2011 Somogyi [48] was the first who employed the property of rotational invariance for handling angular integrals. He did the first systematic calculation of the angular integrals with a parametrization independent of the choice of coordinate axis. Furthermore, his approach is much more straightforward than Neerven's method. Ref. [48] gave the first closed result for the single-massive integral in terms of the Appell function. However, for the double massive integral ref. [48] can only give a Mellin-Barnes representation and no closed formula in terms of hypergeometric functions. Ref. [48] lists the massless integrals up to order $\varepsilon$, the single massive and double massive to finite order. For the double massive integral the order $\varepsilon$ term could not be derived using the Mellin-Barnes methods since it involved complicated triple sums. Unpublished work towards the order $\varepsilon$ by other means is cited to also "not have an analytic answer" [47]. In the present paper we will present this so far missing result in section 3.2.6. 
The closed analytic result for the single massive integral was first used in ref. [68] for the calculation of subtraction terms in QCD jet cross sections at NNLO and more recently in ref. [69] for subtraction terms in Higgs production. The single massive integral found further application in ref. [70] to pin down phase space master integrals. Also the $\mathrm{N}^{3} \mathrm{LO}$ study of Higgs production in ref. [72] employed Somogyi's new results. A brought discussion of techniques for phase-space calculations, emphasizing the benefits of a rotational invariant approach based on Somogyi's methods, is given in ref. [71]. Ref. [59] on the Drell-Yan process at $\mathrm{N}^{3} \mathrm{LO}$ involves both the Neerven integral and Somogyis findings in the determination of phase space integrals in the soft region. Furthermore angular integrals in the Somogyi parametrization make their appearance in the determination of beam and soft functions (see ref. [73]).

However, many recent investigations are still based on the rather old Neerven list. The angular integral in Neerven parametrization appears in [74] and [75] as part of the three particle phase space of single spin asymmetry in $W$-boson production. Ref. [76] employed it for similar purpose. In ref. [61] the angular integral is used in Neerven form and some additional integrals wih higher indices are provided. Ref. [77] employs normalized Neerven integrals for a study of supersymmetry. Further examples of recent uses of Neervens integral list are such diverse topics as the investigation of twist-3 single-spin asymmetry [79], heavy flavour production [80], or the study of Kaluza-Klein gluons in ref. [78]. The present paper is aimed at providing a long overdue update to the still frequently used tool of angular integration for future studies similar to those mentioned here.

The following three perturbative studies are further examples of recent practice where the presented method could have been conveniently applied. Ref. [62] considers the Neerven integral as part of the three-particle phase space of NNLO real corrections to SIDIS at high transverse momentum. The symmetry of single massive integrals is noticed by change of frame, a closed form for the massless integral is given however it is stated that "the integral no longer has a closed form" in the single massive case. They give a detailed description of their partial fractioning algorithm based on Mandelstam identities and provide an extensive list of integrals. A recent paper on $e^{+} e^{-}$-annihilation gives the double massive integral $I_{4}^{(j, l)}$ for $1 \leq j, l \leq 2$ and states "we were not able to find a closed form in $d$ dimensions" [81]. Their eq. (151)-(153) constitute two-point partial fractioning, eq. (154)-(159) is a version of three-point partial fractioning. In a recent publication on QED corrections in exclusive $\bar{B} \rightarrow \bar{K} \ell^{+} \ell^{-}$decay [67], the order $\varepsilon$ of the double massive angular integral was explicitly needed to extract collinear logarithms from soft integrals. Private communication with Somogyi is cited to obtain a result. This showcases the need to improve on the existing literature on angular integrals.

The broad range of applications of the methods for dealing with angular integrals in QCD processes motivates a fresh study of this problem to supplement the toolbox for PSI. In particular, one of the main purposes of our paper is to present the angular integrals in a systematic fashion based on the Somogyi framework using relativistically invariant variables (scalar products of four momenta/velocities) [48].

Since the appearance of the angular integrals over a small number of propagators is intimately connected to partial fractioning, we also provide an algorithm which directly 
leads to the angular integrals in the form introduced in section 3.2. This has the benefit that partial fractioning becomes manifestly invariant under rotation and leads to considerably short expressions without the need to choose suitable coordinate axis, simplifying computer implementation.

The systematic approach offers a cleaner picture on the possible types of integrals appearing. Furthermore, we naturally obtain an expression for the double massive integral in $D$ dimensions with integer coefficients $j, l$ in terms of single massive integrals, not known in the existing literature. The general Neerven integral with integer valued $j$ and $l$ is calculated in $D$ dimensions and expanded to all orders in $\varepsilon$ for the first time. The whole calculation is presented in a pedagogical form to encourage further studies and supplement the existing extensive lists of integrals with straightforward derivations.

The paper is structured as follows. In section 2 we discuss novel ideas for handling of loop tensorial integrals in $D$ dimensions using the basis of orthogonal external momenta. In section 3 we discuss new ideas concerning angular integration in $D$ dimensions. Section 4 contains our conclusions and summary of the main results. Technical details of calculations, a discussion of parial fractioning in PSIs, and tables of angular integrals are placed in appendices.

\section{New formalism for reduction of tensor loop integrals}

In this section we discuss our newly developed formalism for handling of loop tensorial integrals in $D$ dimensions using an orthogonal basis of external momenta. As we stressed in Introduction we develop analytic method for evaluation of one-loop diagrams with its further application to QCD processes at fixed target regime (DY and SIDIS processes, prompt photon and heavy flavor production, etc.). Our method can be extended to arbitrary number of legs in one-loop diagrams and for both massless and massive particles. Development of analytical methods for study of QCD processes is very important for understanding of their different aspects: angular distributions, spin effects, access to the parton distributions. It is also important for performing resummation of large logarithms, verifying of different identities (like Lam-Tung relation) involving hadronic structure functions, behavior of the hadronic amplitudes at different kinematical limits and their expansions in small parameters (like small transverse momentum of photon, etc.). See, e.g., discussion in refs. [42, 43].

In present paper we restrict to consideration of one-loop integral with 2,3 , and 4 external legs and with specific kinematic relevant for QCD processes at fixed target regime. Typical loop diagrams with 2,3 , and 4 external legs are shown in figure 1.

First, we define the 9 possible denominators occurring in these loop integrals:

$$
\begin{array}{lll}
\Delta_{0}=k^{2}, & \Delta_{1}=\left(k+p_{1}\right)^{2}, & \Delta_{2}=\left(k-p_{2}\right)^{2}, \\
\Delta_{4}=(k-q)^{2}, & \Delta_{5}=\left(k+p_{1}-k_{1}\right)^{2}, & \Delta_{6}=\left(k+p_{1}-q\right)^{2}, \\
\Delta_{7}=\left(k+p_{1}+p_{2}\right)^{2}, & \Delta_{8}=(k+q)^{2}, & \Delta_{9}=\left(k+k_{1}\right)^{2} .
\end{array}
$$




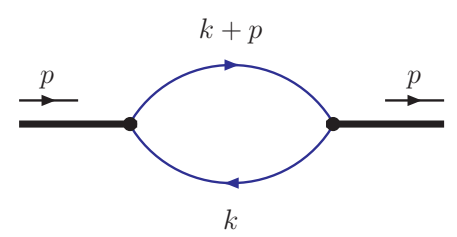

(a)

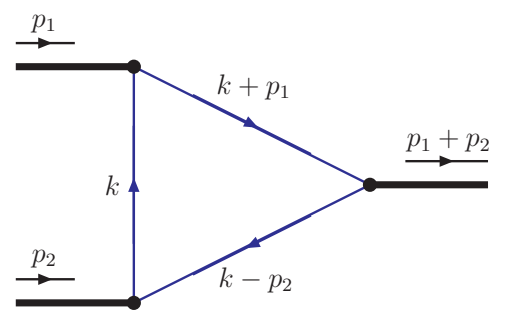

(b)

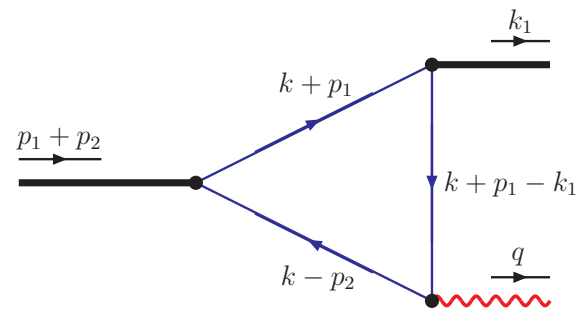

(c)

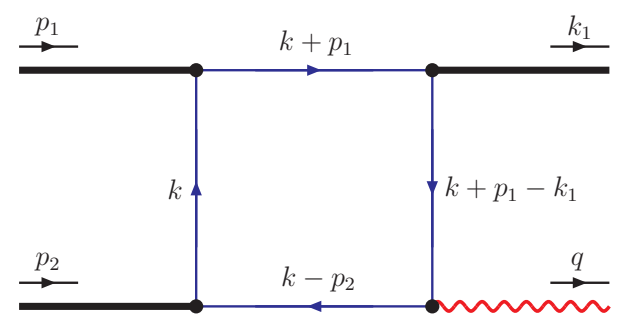

(d)

Figure 1. Loop diagrams: bubble (a), triangle (b) and (c), and box (d).

Our formalism is based on the PV technique. We improve it by expanding the tensor loop integrals in an orthogonal basis of vectors instead of plain external momenta. In case of four external particles the orthogonal basis is specified as following. Let $p_{1}$ and $p_{2}$ be the momenta of incoming massless particles with $p_{1}^{2}=p_{2}^{2}=0$ and let $k_{1}$ and $q$ be the momenta of outcoming particles with massless $k_{1}^{2}=0$ and massive/virtual $q^{2}=Q^{2}$. They obey four-momentum conservation $p_{1}+p_{2}=k_{1}+q$. The set of Mandelstam variables is specified as:

$$
\begin{aligned}
& s=\left(p_{1}+p_{2}\right)^{2}=\left(k_{1}+q\right)^{2}, \\
& t=\left(p_{1}-q\right)^{2}=\left(p_{2}-k_{1}\right)^{2}, \\
& u=\left(p_{1}-k_{1}\right)^{2}=\left(p_{2}-q\right)^{2}, \\
& s+t+u=q^{2}=Q^{2} .
\end{aligned}
$$

Now we define the basis of 3 orthogonal momenta as

$$
P^{\mu}=\left(p_{1}+p_{2}\right)^{\mu}, \quad R^{\mu}=\left(p_{1}-p_{2}\right)^{\mu}, \quad T^{\mu}=k_{1}^{\mu}-P^{\mu} \frac{P k_{1}}{P^{2}}-R^{\mu} \frac{R k_{1}}{R^{2}},
$$


where $P^{2}=-R^{2}=s, T^{2}=-\frac{u t}{s}$ and $P \cdot R=P \cdot T=R \cdot T=0$. Also we introduce the perpendicular metric tensor $g_{\perp}^{\mu \nu}$, which is manifestly orthogonal to all basis momenta:

$$
g_{\perp}^{\mu \nu}=g^{\mu \nu}-\frac{P^{\mu} P^{\nu}}{P^{2}}-\frac{R^{\mu} R^{\nu}}{R^{2}}-\frac{T^{\mu} T^{\nu}}{T^{2}},
$$

which satisfies $g_{\perp}^{\mu \nu} g_{\mu \nu}^{\perp}=D-3, g_{\perp}^{\mu \nu} P_{\mu}=g_{\perp}^{\mu \nu} R_{\mu}=g_{\perp}^{\mu \nu} T_{\mu}=0$. All above formulas can be extended to the case of a massive particle with momentum $k_{1}$. The only difference is that $T^{2}=\frac{Q^{2} k_{1}^{2}-u t}{s}$. The use of such basis considerably simplifies obtaining all scalar functions parameterizing the loop integrals.

Note, that an extension to an arbitrary number of external and massive particles is straightforward. In particular, if we consider the scattering of two particles with momenta $p_{1}$ and $p_{2}$ producing $n$ particles in the final state with momenta $k_{1}, \ldots, k_{n}$, then the orthogonal basis involving $(n+1)$ momenta $\left\{P_{1}, \ldots P_{n+1}\right\}$ is specified as

$$
\begin{aligned}
P_{1}^{\mu} & =P^{\mu}, \quad P=p_{1}+p_{2}, \\
P_{2}^{\mu} & =R^{\mu}-P^{\mu} \frac{P R}{P^{2}}, \quad R=p_{1}-p_{2}, \\
P_{3}^{\mu} & =k_{1}^{\mu}-P_{1}^{\mu} \frac{P_{1} k_{1}}{P_{1}^{2}}-P_{2}^{\mu} \frac{P_{2} k_{2}}{P_{2}^{2}}, \\
& \ldots \\
P_{n+1}^{\mu} & =k_{n-1}^{\mu}-P_{1}^{\mu} \frac{P_{1} k_{n-1}}{P_{1}^{2}}-P_{2}^{\mu} \frac{P_{2} k_{n-1}}{P_{2}^{2}}-\ldots-P_{n}^{\mu} \frac{P_{n} k_{n-1}}{P_{n}^{2}} .
\end{aligned}
$$

For the loop integrals with $n$ propagators and with a product of $m$ external momenta in numerator we use the notation:

$$
I_{i_{1} \ldots i_{n}}^{\alpha_{1} \ldots \alpha_{m}}=\left(i \pi^{2}\right)^{-1} \mu^{4-D} \int d^{D} k \frac{k^{\alpha_{1}} \ldots k^{\alpha_{m}}}{\Delta_{i_{1}} \ldots \Delta_{i_{n}}},
$$

where $\mu$ is the renormalization scale. Below we list the results for bubble, triangle, and box integrals with kinematics [see eqs. (2.2)-(2.4)] specific for the QCD processes at fixed target regime at NNLO.

\subsection{Bubble integrals}

All bubble integrals are expressed through the scalar bubble PV function $B_{0}\left(p^{2}\right)$

$$
\begin{aligned}
B_{0}\left(p^{2}\right) & =\left(i \pi^{2}\right)^{-1} \mu^{4-D} \int d^{D} k \frac{1}{k^{2}(k+p)^{2}} \\
& =\pi^{\frac{D}{2}-2} \frac{\Gamma\left(2-\frac{D}{2}\right) \Gamma^{2}\left(\frac{D}{2}-1\right)}{\Gamma(D-2)}\left(-\frac{p^{2}}{\mu^{2}}\right)^{\frac{D}{2}-2},
\end{aligned}
$$

where $\Gamma(x)$ is the gamma function. The vector bubble integrals are given by:

$$
\begin{aligned}
B_{1}^{\mu}(p) & =\left(i \pi^{2}\right)^{-1} \mu^{4-D} \int d^{D} k \frac{k^{\mu}}{k^{2}(k+p)^{2}}=-\frac{p^{\mu}}{2} B_{0}\left(p^{2}\right) \\
B_{2}^{\mu \nu}(p) & =\left(i \pi^{2}\right)^{-1} \mu^{4-D} \int d^{D} k \frac{k^{\mu} k^{\nu}}{k^{2}(k+p)^{2}}=\frac{1}{4(D-1)}\left(p^{\mu} p^{\nu} D-g^{\mu \nu} p^{2}\right) B_{0}\left(p^{2}\right) \\
& =\frac{1}{4}\left[p^{\mu} p^{\nu}-g_{\perp ; 2}^{\mu \nu} \frac{p^{2}}{D-1}\right] B_{0}\left(p^{2}\right)
\end{aligned}
$$


where $g_{\perp ; 2}^{\mu \nu}=g^{\mu \nu}-p^{\mu} p^{\nu} / p^{2}$ is the perpendicular metric tensor for the bubble diagram with the property:

$$
g_{\perp ; 2}^{\mu \nu} g_{\perp ; 2 ; \mu \nu}=D-1 .
$$

Here and in the following, we introduce three orthogonal metric tensors, one for each specific diagram — bubble, triangle, and box — which will be labeled by the indices 2, 3, and 4, respectively. Also, we will see that the following normalization condition for the metric tensors holds:

$$
g_{\perp ; n}^{\mu \nu} g_{\perp ; n ; \mu \nu}=D+1-n .
$$

For specific choices of momenta the scalar bubble integrals $I_{i j} \equiv B_{0}^{i j}$ read:

$$
\begin{aligned}
& B_{0}(0)=B_{0}^{01}=B_{0}^{02}=B_{0}^{03}=B_{0}^{09}, \\
& B_{0}(u)=B_{0}^{05}, \quad B_{0}(t)=B_{0}^{06}, \quad B_{0}(s)=B_{0}^{07}, \quad B_{0}\left(Q^{2}\right)=B_{0}^{04}=B_{0}^{08} .
\end{aligned}
$$

Tensorial bubble integrals for specific momenta follow from eqs. (2.8) and (2.9). Note that in case of bubble diagrams we work with a single transverse vector. Next, in case of triangle and box diagrams we will use two and three transverse vectors. However, these perpendicular vectors can be always expanded in a linear combination of the vectors of the basis (2.3).

\subsection{Triangle integrals}

For the triangle integrals (scalar, vector, tensor rank-2) we use the following definitions, respectively:

$$
\begin{aligned}
I_{i j k} & =\left(i \pi^{2}\right)^{-1} \mu^{4-D} \int d^{D} k \frac{1}{\Delta_{i} \Delta_{j} \Delta_{k}}, \\
I_{i j k}^{\mu} & =\left(i \pi^{2}\right)^{-1} \mu^{4-D} \int d^{D} k \frac{k^{\mu}}{\Delta_{i} \Delta_{j} \Delta_{k}}, \\
I_{i j k}^{\mu \nu} & =\left(i \pi^{2}\right)^{-1} \mu^{4-D} \int d^{D} k \frac{k^{\mu} k^{\nu}}{\Delta_{i} \Delta_{j} \Delta_{k}} .
\end{aligned}
$$

The scalar integrals $I_{i j k} \equiv C_{0}^{i j k}$ obey the following relations and are expressed through the $\mathrm{PV}$ triangle scalar integrals $C_{0}\left(s_{1}, s_{2}\right)$ as:

$$
\begin{aligned}
C_{0}(s) & =C_{0}^{012}=C_{0}^{017}, \\
C_{0}(t) & =C_{0}^{023}=C_{0}^{026}=C_{0}^{069}, \\
C_{0}(u) & =C_{0}^{015}=C_{0}^{019}, \\
C_{0}\left(s, Q^{2}\right) & =C_{0}^{038}=C_{0}^{049}=C_{0}^{078}=C_{0}^{079}, \\
C_{0}\left(t, Q^{2}\right) & =C_{0}^{016}=C_{0}^{018}=C_{0}^{046}, \\
C_{0}\left(u, Q^{2}\right) & =C_{0}^{024}=C_{0}^{025}=C_{0}^{035}=C_{0}^{058} .
\end{aligned}
$$

Here, the integral $C_{0}\left(s_{1}, s_{2}, s_{3}\right)$ is defined as

$$
C_{0}\left(s_{1}, s_{2}, s_{3}\right)=\left(i \pi^{2}\right)^{-1} \mu^{4-D} \int d^{D} k \frac{1}{k^{2}\left(k+l_{1}\right)^{2}\left(k+l_{2}\right)^{2}},
$$

where $s_{1}=l_{1}^{2}, s_{2}=l_{2}^{2}, s_{3}=l_{3}^{2}=\left(l_{1}-l_{2}\right)^{2}$, and $2 l_{1} l_{2}=s_{1}+s_{2}-s_{3}$. 
It is fully symmetric under all permutations of its arguments

$$
\begin{aligned}
C_{0}\left(s_{1}, s_{2}, s_{3}\right) & =C_{0}\left(s_{1}, s_{3}, s_{2}\right)=C_{0}\left(s_{2}, s_{3}, s_{1}\right)=C_{0}\left(s_{2}, s_{1}, s_{3}\right) \\
& =C_{0}\left(s_{3}, s_{1}, s_{2}\right)=C_{0}\left(s_{3}, s_{2}, s_{1}\right) .
\end{aligned}
$$

In specific limit, when one of the arguments is equal to zero, it is expressed through the bubble PV integral $B_{0}(s)$ as:

$$
C_{0}\left(s_{1}, s_{2}, 0\right)=\frac{2(D-3)}{4-D} \frac{B_{0}\left(s_{1}\right)-B_{0}\left(s_{2}\right)}{s_{1}-s_{2}} .
$$

Now we consider tensorial triangle integrals. In contrast to the bubble diagram case, here we introduce a "perpendicular basis" for the triangle master integral containing two vectors. These two can be related to our "orthogonal basis" containing the momenta $P, R, T$ used to parametrize all considered types of loop diagrams - bubble, triangle, box.

The master vector triangle integral reads

$$
C_{1}^{\mu}\left(s_{1}, s_{2}, s_{3}\right)=\left(i \pi^{2}\right)^{-1} \mu^{4-D} \int d^{D} k \frac{k^{\mu}}{k^{2}\left(k+l_{1}\right)^{2}\left(k+l_{2}\right)^{2}} .
$$

For handling triangle diagrams the perpendicular basis reads:

$$
\begin{aligned}
& l_{1 \perp}^{\mu}=l_{1}^{\mu}-l_{2}^{\mu} \frac{l_{1}^{2}}{l_{1} l_{2}}, \\
& l_{2 \perp}^{\mu}=l_{2}^{\mu}-l_{1}^{\mu} \frac{l_{2}^{2}}{l_{1} l_{2}},
\end{aligned}
$$

with the following properties:

$$
\begin{aligned}
l_{i \perp} \cdot l_{i} & =0, & i=1,2, \\
l_{i \perp}^{2} & =-\frac{l_{i}^{2}}{4\left(l_{1} l_{2}\right)^{2}} \lambda\left(l_{1}^{2}, l_{2}^{2}, l_{3}^{2}\right), & i=1,2, \\
l_{1 \perp} \cdot l_{2 \perp} & =\frac{\lambda\left(l_{1}^{2}, l_{2}^{2}, l_{3}^{2}\right)}{4 l_{1} l_{2}}, & \\
\left(l_{1 \perp} \cdot l_{2 \perp}\right)^{2} & =\frac{\left(l_{1} l_{2}\right)^{2}}{l_{1}^{2} l_{2}^{2}} l_{1 \perp}^{2} l_{2 \perp}^{2}, &
\end{aligned}
$$

where

$$
\lambda(x, y, z)=x^{2}+y^{2}+z^{2}-2 x y-2 y z-2 x z,
$$

is the kinematic triangle Källen function. In addition, to accompany the perpendicular basis, we introduce orthogonal metric tensor $g_{\perp ; 3}^{\mu \nu}$, which is defined as

$$
g_{\perp ; 3}^{\mu \nu}=g^{\mu \nu}-\frac{l_{1 \perp}^{\mu} l_{\perp \perp}^{\nu} l_{2}^{2}}{\left(l_{1 \perp} l_{2 \perp}\right)^{2}}-\frac{l_{2 \perp}^{\mu} l_{2 \perp}^{\nu} l_{1}^{2}}{\left(l_{1 \perp} l_{2 \perp}\right)^{2}}-\frac{\left(l_{1 \perp}^{\mu} l_{2 \perp}^{\nu}+l_{2 \perp}^{\mu} l_{1 \perp}^{\nu}\right) l_{1} l_{2}}{\left(l_{1 \perp} l_{2 \perp}\right)^{2}},
$$

and has the following properties

$$
g_{\perp ; 3}^{\mu \nu} \cdot l_{i \perp ; \mu}=g_{\perp ; 3}^{\mu \nu} \cdot l_{i \perp ; \nu}=g_{\perp ; 3}^{\mu \nu} \cdot l_{i ; \mu}=g_{\perp ; 3}^{\mu \nu} \cdot l_{i ; \nu}=0, \quad i=1,2,
$$

and

$$
g_{\perp ; 3}^{\mu \nu} \cdot g_{\perp ; 3 ; \mu \nu}=D-2 .
$$

Note, that such choice of basis is free of soft singularities occurring in the limit $l_{1}^{2}$ and/or $l_{2}^{2}=0$. In particular, in this limit $l_{i \perp} \rightarrow l_{i}$. Also it gives the straightforward results for 
the scalar functions in the expansion of tensorial triangle diagrams, i.e. without the need of solving a system of equations like in the original PV method.

The expansion of the integral $C_{1}^{\mu}\left(s_{1}, s_{2}, s_{3}\right)$ in the basis reads

$$
C_{1}^{\mu}\left(s_{1}, s_{2}, s_{3}\right)=l_{1 \perp}^{\mu} C_{1 ; l 1}+l_{2 \perp}^{\mu} C_{1 ; l 2},
$$

where the scalar functions $C_{1 ; l 1}$ and $C_{1 ; l 2}$ are fixed by contraction of our loop integral with $l_{2}$ and $l_{1}$, respectively:

$$
\begin{aligned}
& C_{1 ; l 1}\left(s_{1}, s_{2}, s_{3}\right)=\frac{1}{l_{1 \perp} l_{2 \perp}} C_{1}^{\mu}\left(s_{1}, s_{2}, s_{3}\right) l_{2 \mu} \\
& C_{1 ; l 2}\left(s_{1}, s_{2}, s_{3}\right)=\frac{1}{l_{1 \perp} l_{2 \perp}} C_{1}^{\mu}\left(s_{1}, s_{2}, s_{3}\right) l_{1 \mu} .
\end{aligned}
$$

Analytic expressions for the functions $C_{1 ; l 1}$ and $C_{1 ; l 2}$ read

$$
\begin{aligned}
& C_{1 ; l 1}\left(s_{1}, s_{2}, s_{3}\right)=C_{1}\left(s_{1}, s_{2}, s_{3}\right)=\frac{2 l_{1} l_{2}}{\lambda}\left[B_{0}\left(s_{1}\right)-B_{0}\left(s_{3}\right)-s_{2} C_{0}\left(s_{1}, s_{2}, s_{3}\right)\right], \\
& C_{1 ; l 2}\left(s_{1}, s_{2}, s_{3}\right)=C_{1}\left(s_{2}, s_{1}, s_{3}\right)=\frac{2 l_{1} l_{2}}{\lambda}\left[B_{0}\left(s_{2}\right)-B_{0}\left(s_{3}\right)-s_{1} C_{0}\left(s_{1}, s_{2}, s_{3}\right)\right] .
\end{aligned}
$$

Note, that $C_{1 ; l 1}$ and $C_{1 ; l 2}$ are related via exchange of momenta $l_{1} \leftrightarrow l_{2}$ or $s_{1} \leftrightarrow s_{2}$.

By analogy we pin down triangle tensor rank-2 integral

$$
\begin{aligned}
C_{2}^{\mu \nu}\left(s_{1}, s_{2}, s_{3}\right) & =\left(i \pi^{2}\right)^{-1} \mu^{4-D} \int d^{D} k \frac{k^{\mu} k^{\nu}}{k^{2}\left(k+l_{1}\right)^{2}\left(k+l_{2}\right)^{2}} \\
& =g_{\perp ; 3}^{\mu \nu} C_{2 ; g}+l_{1 \perp}^{\mu} l_{1 \perp}^{\nu} C_{2 ; l_{1} l_{1}}+l_{2 \perp}^{\mu} l_{2 \perp}^{\nu} C_{2 ; l_{2} l_{2}}+\left(l_{1 \perp}^{\mu} l_{2 \perp}^{\nu}+l_{2 \perp}^{\mu} l_{1 \perp}^{\nu}\right) C_{2 ; l_{1} l_{2}} .
\end{aligned}
$$

Again, we can easily and directly extract the scalar functions $C_{2 ; 11 l 1}, C_{2 ; l 2 l 2}$, and $C_{2 ; 11 l 2}$ using the formulas

$$
\begin{aligned}
C_{2 ; l_{1} l_{1}} & =\frac{1}{\left(l_{1 \perp} l_{2 \perp}\right)^{2}} C_{2}^{\mu \nu}\left(s_{1}, s_{2}, s_{3}\right) l_{2 \mu} l_{2 \nu} \\
C_{2 ; l_{2} l_{2}} & =\frac{1}{\left(l_{1 \perp} l_{2 \perp}\right)^{2}} C_{2}^{\mu \nu}\left(s_{1}, s_{2}, s_{3}\right) l_{1 \mu} l_{1 \nu} \\
C_{2 ; l_{1} l_{2}} & =\frac{1}{\left(l_{1 \perp} l_{2 \perp}\right)^{2}} C_{2}^{\mu \nu}\left(s_{1}, s_{2}, s_{3}\right) l_{1 \mu} l_{2 \nu} \\
C_{2 ; g} & =\frac{1}{D-2} C_{2}^{\mu \nu}\left(s_{1}, s_{2}, s_{3}\right) g_{\perp ; 3 ; \mu \nu}
\end{aligned}
$$

Analytic expressions for these functions read

$$
\begin{aligned}
& C_{2 ; l_{1} l_{1}}\left(s_{1}, s_{2}, s_{3}\right)=\frac{4\left(l_{1} l_{2}\right)^{2}}{\lambda^{2}} {\left[-l_{1} l_{2} B_{0}\left(s_{1}\right)+\left(s_{2}+l_{1} l_{2}\right) B_{0}\left(s_{3}\right)-\frac{\lambda s_{2}}{2 l_{1} l_{2}} C_{1 ; l_{1}}\left(s_{1}, s_{2}, s_{3}\right)\right], } \\
& C_{2 ; l_{2} l_{2}}\left(s_{1}, s_{2}, s_{3}\right)=\frac{4\left(l_{1} l_{2}\right)^{2}}{\lambda^{2}}\left[-l_{1} l_{2} B_{0}\left(s_{2}\right)+\left(s_{1}+l_{1} l_{2}\right) B_{0}\left(s_{3}\right)-\frac{\lambda s_{1}}{2 l_{1} l_{2}} C_{1 ; l_{2}}\left(s_{1}, s_{2}, s_{3}\right)\right], \\
& C_{2 ; l_{1} l_{2}}\left(s_{1}, s_{2}, s_{3}\right)=\frac{2\left(l_{1} l_{2}\right)^{2}}{\lambda^{2}} {\left[-s_{1} B_{0}\left(s_{1}\right)-s_{2} B_{0}\left(s_{2}\right)+\left(s_{1}+s_{2}+2 l_{1} l_{2}\right) B_{0}\left(s_{3}\right)\right.} \\
&\left.-\frac{\lambda}{2 l_{1} l_{2}}\left(s_{1} C_{1 ; l_{1}}\left(s_{1}, s_{2}, s_{3}\right)+s_{2} C_{1 ; l_{2}}\left(s_{1}, s_{2}, s_{3}\right)\right)\right],
\end{aligned}
$$




$$
\begin{aligned}
C_{2 ; g}\left(s_{1}, s_{2}, s_{3}\right)=\frac{1}{D-2}[ & B_{0}\left(s_{3}\right)+\frac{\lambda}{4\left(l_{1} l_{2}\right)^{2}}\left(s_{1} C_{2 ; l_{1} l_{1}}\left(s_{1}, s_{2}, s_{3}\right)+s_{2} C_{2 ; l_{2} l_{2}}\left(s_{1}, s_{2}, s_{3}\right)\right) \\
& \left.-\frac{\lambda}{2 l_{1} l_{2}} C_{2 ; l_{1} l_{2}}\left(s_{1}, s_{2}, s_{3}\right)\right] .
\end{aligned}
$$

Note, that the functions $C_{2 ; l_{1} l_{1}}\left(s_{1}, s_{2}, s_{3}\right)$ and $C_{2 ; l_{1} l_{1}}\left(s_{1}, s_{2}, s_{3}\right)$ are symmetric under exchange of first two arguments $s_{1} \leftrightarrow s_{2}$. In appendix A we present the solutions for the scalar functions $C_{2 ; g}, C_{2 ; l_{1} l_{1}}, C_{2 ; l_{2} l_{2}}$, and $C_{2 ; l_{1} l_{2}}$ for specific choice of momenta (including soft limits). Finally, we note that the perpendicular basis vector $l_{i \perp}$ can be easily expressed through the basis vectors $P, R$, and $T$.

Finally we present analytic results for triangle tensor rank-3 integral

$$
\begin{aligned}
C_{3}^{\mu \nu \alpha}\left(s_{1}, s_{2}, s_{3}\right)= & \left(i \pi^{2}\right)^{-1} \mu^{4-D} \int d^{D} k \frac{k^{\mu} k^{\nu} k^{\alpha}}{k^{2}\left(k+l_{1}\right)^{2}\left(k+l_{2}\right)^{2}} \\
= & g_{\perp ; l_{1}}^{\mu \nu \alpha} C_{3 ; g l_{1}}+g_{\perp ; l_{\perp}}^{\mu \nu \alpha} C_{3 ; g l_{2}} \\
& +\left\{l_{1 \perp} l_{1 \perp} l_{1 \perp}\right\}^{\mu \nu \alpha} C_{3 ; l_{1} l_{1} l_{1}}+\left\{l_{2 \perp} l_{2 \perp} l_{2 \perp}\right\}^{\mu \nu \alpha} C_{3 ; l_{2} l_{2} l_{2}} \\
& +\left\{l_{1 \perp} l_{1 \perp} l_{2 \perp}\right\}^{\mu \nu \alpha} C_{3 ; l_{1} l_{1} l_{2}}+\left\{l_{2 \perp} l_{2 \perp} l_{1 \perp}\right\}^{\mu \nu \alpha} C_{3 ; l_{2} l_{2} l_{1}},
\end{aligned}
$$

where

$$
\begin{aligned}
g_{\perp ; l_{i \perp}}^{\mu \nu \alpha} & =g_{\perp ; 3}^{\mu \nu} l_{i \perp}^{\alpha}+g_{\perp ; 3}^{\mu \alpha} l_{i \perp}^{\beta}+g_{\perp ; 3}^{\mu \beta} l_{i \perp}^{\alpha}, \\
\{A A A\}^{\mu \nu \alpha} & =A^{\mu} A^{\nu} A^{\alpha} \\
\{A A B\}^{\mu \nu \alpha} & =A^{\mu} A^{\nu} B^{\alpha}+A^{\nu} A^{\alpha} B^{\mu}+A^{\alpha} A^{\mu} B^{\nu} .
\end{aligned}
$$

The scalar functions $C_{3 ; g l_{1}}, C_{3 ; g l_{2}}, C_{3 ; l_{1} l_{1} l_{1}}, C_{3 ; l_{2} l_{2} l_{2}}, C_{3 ; l_{1} l_{1} l_{2}}$, and $C_{3 ; l_{2} l_{2} l_{1}}$ are given by

$$
\begin{aligned}
C_{3 ; g l_{1}}= & \frac{4 l_{1} l_{2}}{(D-2) \lambda\left(l_{1}^{2}, l_{2}^{2}, l_{3}^{2}\right)} g_{\mu \nu ; 3} l_{2 \alpha} C_{3}^{\mu \nu \alpha}\left(s_{1}, s_{2}, s_{3}\right) \\
= & -\frac{l_{1} l_{2}}{(D-2) \lambda\left(l_{1}^{2}, l_{2}^{2}, l_{3}^{2}\right)} \frac{B_{0}\left(l_{3}^{2}\right)}{2} l_{2}\left(2 l_{2}+l_{3}\right), \\
C_{3 ; l_{1} l_{1} l_{1}}= & \frac{64\left(l_{1} l_{2}\right)^{3}}{\lambda^{3}\left(l_{1}^{2}, l_{2}^{2}, l_{3}^{2}\right)} l_{2 \mu} l_{2 \nu} l_{2 \alpha} C_{3}^{\mu \nu \alpha}\left(s_{1}, s_{2}, s_{3}\right) \\
= & \frac{8\left(l_{1} l_{2}\right)^{3}}{\lambda^{3}\left(l_{1}^{2}, l_{2}^{2}, l_{3}^{2}\right)}\left[B_{0}\left(l_{2}^{2}\right) \frac{D\left(l_{1} l_{2}\right)^{2}-l_{1}^{2} l_{2}^{2}}{D-1}-B_{0}\left(l_{3}^{2}\right) \frac{D\left(l_{1} l_{3}\right)^{2}-l_{1}^{2} l_{3}^{2}}{D-1}\right. \\
C_{3 ; l_{1} l_{1} l_{2}}= & \frac{64\left(l_{1} l_{2}\right)^{3}}{\lambda^{3}\left(l_{1}^{2}, l_{2}^{2}, l_{3}^{2}\right)} l_{2 \mu} l_{2 \nu} l_{1 \alpha} C_{3}^{\mu \nu \alpha}\left(s_{1}, s_{2}, s_{3}\right) \\
= & \frac{8\left(l_{1} l_{2}\right)^{3}}{\lambda^{3}\left(l_{1}^{2}, l_{2}^{2}, l_{3}^{2}\right)}\left[B_{0}\left(l_{2}^{2}\right) l_{2}^{4}-B_{0}\left(l_{3}^{2}\right)\left(l_{2}^{2}\left(2 l_{2}+l 3\right)\right)^{2}\right. \\
& \left.\quad-C_{2 ; l_{1} l_{1}} \frac{l_{1}^{2} \lambda^{2}\left(l_{1}^{2}, l_{2}^{2}, l_{3}^{2}\right)}{4\left(l_{1} l_{2}\right)^{2}}\right] .
\end{aligned}
$$


Here functions $C_{3 ; g l_{2}}, C_{3 ; l_{2} l_{2} l_{2}}, C_{3 ; l_{2} l_{2} l_{1}}$ are obtainded from functions $C_{3 ; g l_{1}}, C_{3 ; l_{1} l_{1} l_{1}}, C_{3 ; l_{1} l_{1} l_{2}}$ under replacements $l_{1} \rightarrow l_{2}, l_{2} \rightarrow l_{1}, l_{3} \rightarrow-l_{3}$. As before, the perpendicular basis vector $l_{i \perp}$ is easily expressed through the basis vectors $P, R$, and $T$.

\subsection{Box integrals}

A typical box integral is shown in figure 1(d) [the others are obtained by permutation of external momenta in the propagators].

We start with the calculation of the scalar integral

$$
D_{0}\left(s, u, Q^{2}\right) \equiv I_{0125}=\left(i \pi^{2}\right)^{-1} \mu^{4-D} \int d^{D} k \frac{1}{\Delta_{0} \Delta_{1} \Delta_{2} \Delta_{5}} .
$$

To pin down this integral we use Feynman $\alpha$-parametrization (for calculation of fully massless integral with $Q^{2}=0$ see ref. [24]). The final results can be written in terms of the Gauss hypergeometric function ${ }_{2} F_{1}(a, b, c, z)$ [see details of calculation in appendix B.1]. The expression for the box integral reads (see also refs. [20, 22, 35]):

$$
\begin{aligned}
D_{0}\left(s, u, Q^{2}\right)= & -\frac{1}{s u}\left[\pi^{\frac{D}{2}-2} \frac{\Gamma\left(2-\frac{D}{2}\right) \Gamma^{2}\left(\frac{D}{2}-2\right)}{\Gamma(D-4)}\right] \\
& \times\left[\left(-\frac{s}{\mu^{2}}\right)^{\frac{D}{2}-2}{ }_{2} F_{1}\left(1, \frac{D}{2}-2, \frac{D}{2}-1,-\frac{t}{u}\right)\right. \\
& +\left(-\frac{u}{\mu^{2}}\right)^{\frac{D}{2}-2}{ }_{2} F_{1}\left(1, \frac{D}{2}-2, \frac{D}{2}-1,-\frac{t}{s}\right) \\
& \left.-\left(-\frac{Q^{2}}{\mu^{2}}\right)^{\frac{D}{2}-2}{ }_{2} F_{1}\left(1, \frac{D}{2}-2, \frac{D}{2}-1,-\frac{Q^{2} t}{s u}\right)\right] \\
= & -\frac{4}{s u}\left[\frac{D-3}{D-4}\right]\left[B_{0}(s){ }_{2} F_{1}\left(1, \frac{D}{2}-2, \frac{D}{2}-1,-\frac{t}{u}\right)\right. \\
& +B_{0}(u){ }_{2} F_{1}\left(1, \frac{D}{2}-2, \frac{D}{2}-1,-\frac{t}{s}\right) \\
& \left.-B_{0}\left(Q^{2}\right){ }_{2} F_{1}\left(1, \frac{D}{2}-2, \frac{D}{2}-1,-\frac{Q^{2} t}{s u}\right)\right] .
\end{aligned}
$$

Note that in the on-shell limit $Q^{2}=s+t+u=0$ we reproduce the result for the scalar box given in ref. [24]. $\varepsilon$ expansion of the scalar box diagram is discussed in detail in appendix B.1. The results for the other scalar box integrals are obtained via permutation of Mandelstam variables $(s, t, u)$. Here we display important symmetry properties of the scalar box integral:

$$
\begin{aligned}
D_{0}\left(s, u, Q^{2}\right) & =D_{0}\left(u, s, Q^{2}\right)=I_{0125}, \\
D_{0}\left(s, t, Q^{2}\right) & =D_{0}\left(t, s, Q^{2}\right)=I_{0126}, \\
D_{0}\left(u, t, Q^{2}\right) & =D_{0}\left(t, u, Q^{2}\right)=I_{0235} .
\end{aligned}
$$


Now we are in the position to derive the expressions for the tensorial box integrals (with one, two, and three loop momentum vectors in numerator) using Lorentz covariance in the orthogonal basis. The loop integrals will be expanded in terms of scalar bubble and triangle PV functions.

We start with vector integrals:

$$
\begin{aligned}
D_{1}^{\mu}\left(s, u, Q^{2}\right) & =\left(i \pi^{2}\right)^{-1} \mu^{4-D} \int d^{D} k \frac{k^{\mu}}{\Delta_{0} \Delta_{1} \Delta_{2} \Delta_{5}} \\
& =P^{\mu} D_{1 P}^{0125}+R^{\mu} D_{1 R}^{0125}+T^{\mu} D_{1 T}^{0125}, \\
D_{1}^{\mu}\left(s, t, Q^{2}\right) & =\left(i \pi^{2}\right)^{-1} \mu^{4-D} \int d^{D} k \frac{k^{\mu}}{\Delta_{0} \Delta_{1} \Delta_{2} \Delta_{6}} \\
& =P^{\mu} D_{1 P}^{0126}+R^{\mu} D_{1 R}^{0126}+T^{\mu} D_{1 T}^{0126}, \\
D_{1}^{\mu}\left(t, u, Q^{2}\right) & =\left(i \pi^{2}\right)^{-1} \mu^{4-D} \int d^{D} k \frac{k^{\mu}}{\Delta_{0} \Delta_{2} \Delta_{3} \Delta_{5}} \\
& =P^{\mu} D_{1 P}^{0235}+R^{\mu} D_{1 R}^{0235}+T^{\mu} D_{1 T}^{0235},
\end{aligned}
$$

where $D_{1 F}^{i j k l}$ are the scalar functions, which are obtained by contraction of the vector integrals $D_{1}^{\mu}\left(s_{1}, s_{2}, Q^{2}\right)$ with corresponding Lorentz structure. In contrast to the original PV method, there remains no system of equations to be solved. E.g., the function $D_{1 P}^{0125}$ is simply fixed as

$$
D_{1 P}^{0125}=\frac{1}{P^{2}} P_{\mu} \cdot D_{1}^{\mu}\left(s, u, Q^{2}\right) .
$$

Then we use the fractioning identity

$$
2 k P=\Delta_{1}-\Delta_{2},
$$

to reduce the number of factors in the denominator of $D_{1}^{\mu}\left(s, u, Q^{2}\right)$. Using eq. (2.43) we get an expression for $D_{1 P}^{0125}$ as linear combination of known scalar triangle integrals:

$$
D_{1 P}^{0125}=\frac{1}{2}\left[C_{0}^{025}-C_{0}^{015}\right]=\frac{1}{2}\left[C_{0}\left(u, Q^{2}\right)-C_{0}(u)\right] .
$$

By analogy we pin down all scalar functions occurring in eqs. (2.39)-(2.41), which are listed in appendix B.2. Also there we give the set of the fractional identities needed to simplify the box diagrams.

The tensor rank-2 box integrals read:

$$
\begin{aligned}
D_{2}^{\mu \nu}\left(s, u, Q^{2}\right)= & \left(i \pi^{2}\right)^{-1} \mu^{4-D} \int d^{D} k \frac{k^{\mu} k^{\nu}}{\Delta_{0} \Delta_{1} \Delta_{2} \Delta_{5}} \\
= & g_{\perp}^{\mu \nu} D_{2 g}^{0125}+\{P P\}^{\mu \nu} D_{2 P P}^{0125}+\{R R\}^{\mu \nu} D_{2 R R}^{0125}+\{T T\}^{\mu \nu} D_{2 T T}^{0125} \\
& +\{P R\}^{\mu \nu} D_{2 P R}^{0125}+\{P T\}^{\mu \nu} D_{2 P T}^{0125}+\{R T\}^{\mu \nu} D_{2 R T}^{0125}, \\
D_{2}^{\mu \nu}\left(s, t, Q^{2}\right)= & \left(i \pi^{2}\right)^{-1} \mu^{4-D} \int d^{D} k \frac{k^{\mu} k^{\nu}}{\Delta_{0} \Delta_{1} \Delta_{2} \Delta_{6}} \\
= & g_{\perp}^{\mu \nu} D_{2 g}^{0126}+\{P P\}^{\mu \nu} D_{2 P P}^{0126}+\{R R\}^{\mu \nu} D_{2 R R}^{0126}+\{T T\}^{\mu \nu} D_{2 T T}^{0126} \\
& +\{P R\}^{\mu \nu} D_{2 P R}^{0126}+\{P T\}^{\mu \nu} D_{2 P T}^{0126}+\{R T\}^{\mu \nu} D_{2 R T}^{0126},
\end{aligned}
$$




$$
\begin{aligned}
D_{2}^{\mu \nu}\left(t, u, Q^{2}\right)= & \left(i \pi^{2}\right)^{-1} \mu^{4-D} \int d^{D} k \frac{k^{\mu} k^{\nu}}{\Delta_{0} \Delta_{2} \Delta_{3} \Delta_{5}} \\
= & g_{\perp}^{\mu \nu} D_{2 g}^{0235}+\{P P\}^{\mu \nu} D_{2 P P}^{0235}+\{R R\}^{\mu \nu} D_{2 R R}^{0235}+\{T T\}^{\mu \nu} D_{2 T T}^{0235} \\
& +\{P R\}^{\mu \nu} D_{2 P R}^{0235}+\{P T\}^{\mu \nu} D_{2 P T}^{0235}+\{R T\}^{\mu \nu} D_{2 R T}^{0235}
\end{aligned}
$$

where $\{A A\}^{\mu \nu}=A^{\mu} A^{\nu}$ and $\{A B\}^{\mu \nu}=A^{\mu} B^{\nu}+A^{\nu} B^{\mu}$. We handle tensor rank-2 box integrals analogous to vector integrals. In particular, we fix the scalar functions $D_{2 F}^{i j k l}$ occurring in the expansions (2.45)-(2.47) by multiplying the corresponding loop integral with the corresponding Lorenz structures $g_{\perp}^{\mu \nu}, P^{\mu} P^{\nu}, R^{\mu} R^{\nu}$, and $T^{\mu} T^{\nu}$, respectively. We do not need to solve any system of equations as in the original PV method. Again, as for vectorial box integrals we use the fractioning identities (see appendix B.2). In particular, the results for the $D_{2 g}^{0125}, D_{2 P P}^{0125}, D_{2 P R}^{0125}$, and $D_{2 T T}^{0125}$ functions read:

$$
\begin{aligned}
D_{2 P P}^{0125}=\frac{1}{P^{4}} P_{\mu} P_{\nu} \cdot D_{2}^{\mu \nu}\left(s, u, Q^{2}\right)= & \frac{1}{2 s}\left[C_{1 P}^{025}-C_{1 P}^{015}\right] \\
D_{2 R R}^{0125}=\frac{1}{R^{4}} R_{\mu} R_{\nu} \cdot D_{2}^{\mu \nu}\left(s, u, Q^{2}\right)=\frac{1}{2 s}[ & \left.C_{1 R}^{025}+C_{1 R}^{015}-2 C_{1 R}^{078}+C_{0}^{025}\right], \\
D_{2 T T}^{0125}=\frac{1}{T^{4}} T_{\mu} T_{\nu} \cdot D_{2}^{\mu \nu}\left(s, u, Q^{2}\right)=-\frac{s}{2 u t}\left[u D_{1 T}^{0125}-C_{1 T}^{015} \frac{u}{s}\right. & \left.+C_{1 T}^{025} \frac{s+t}{s}+C_{1 T}^{078} \frac{u-t}{s}\right], \\
D_{2 g}^{0125}=\frac{1}{D-3} g_{\mu \nu}^{\perp} \cdot D_{2}^{\mu \nu}\left(s, u, Q^{2}\right)=\frac{1}{D-3}\left[C_{0}^{078}-s D_{2 P P}^{0125}\right. & \left.+s D_{2 R R}^{0125}+\frac{u t}{s} D_{2 T T}^{0125}\right] .
\end{aligned}
$$

The tensorial rank-3 box integrals read:

$$
\begin{aligned}
D_{3}^{\mu \nu \alpha}\left(s, u, Q^{2}\right)= & \left(i \pi^{2}\right)^{-1} \mu^{4-D} \int d^{D} k \frac{k^{\mu} k^{\nu} k^{\alpha}}{\Delta_{0} \Delta_{1} \Delta_{2} \Delta_{5}}=g_{\perp P}^{\mu \nu \alpha} D_{3 g P}^{0125}+g_{\perp R}^{\mu \nu \alpha} D_{3 g R}^{0125}+g_{\perp T}^{\mu \nu \alpha} D_{3 g T}^{0125} \\
& +\{P P P\}^{\mu \nu \alpha} D_{3 P P P}^{0125}+\{R R R\}^{\mu \nu \alpha} D_{3 R R R}^{0125}+\{T T T\}^{\mu \nu \alpha} D_{3 T T T}^{0125} \\
& +\{P P R\}^{\mu \nu \alpha} D_{3 P P R}^{0125}+\{P P T\}^{\mu \nu \alpha} D_{3 P P T}^{0125}+\{R R P\}^{\mu \nu \alpha} D_{3 R R P}^{0125} \\
& +\{R R T\}^{\mu \nu \alpha} D_{3 R R T}^{0125}+\{T T P\}^{\mu \nu \alpha} D_{3 T T P}^{0125}+\{T T R\}^{\mu \nu \alpha} D_{3 T T R}^{0125} \\
& +\{P R T\}^{\mu \nu \alpha} D_{3 P R T}^{0125}, \\
D_{3}^{\mu \nu \alpha}\left(s, t, Q^{2}\right)= & \left(i \pi^{2}\right)^{-1} \mu^{4-D} \int d^{D} k \frac{k^{\mu} k^{\nu} k^{\alpha}}{\Delta_{0} \Delta_{1} \Delta_{2} \Delta_{6}}=g_{\perp P}^{\mu \nu \alpha} D_{3 g P}^{0126}+g_{\perp R}^{\mu \nu \alpha} D_{3 g R}^{0126}+g_{\perp T}^{\mu \nu \alpha} D_{3 g T}^{0126} \\
& +\{P P P\}^{\mu \nu \alpha} D_{3 P P P}^{0126}+\{R R R\}^{\mu \nu \alpha} D_{3 R R R}^{0126}+\{T T T\}^{\mu \nu \alpha} D_{3 T T T}^{0126} \\
& +\{P P R\}^{\mu \nu \alpha} D_{3 P P R}^{0126}+\{P P T\}^{\mu \nu \alpha} D_{3 P P T}^{0126}+\{R R P\}^{\mu \nu \alpha} D_{3 R R P}^{0126} \\
& +\{R R T\}^{\mu \nu \alpha} D_{3 R R T}^{0126}+\{T T P\}^{\mu \nu \alpha} D_{3 T T P}^{0126}+\{T T R\}^{\mu \nu \alpha} D_{3 T T R}^{0126} \\
& +\{P R T\}^{\mu \nu \alpha} D_{3 P R T}^{0126},
\end{aligned}
$$




$$
\begin{aligned}
D_{3}^{\mu \nu \alpha}\left(t, u, Q^{2}\right)= & \left(i \pi^{2}\right)^{-1} \mu^{4-D} \int d^{D} k \frac{k^{\mu} k^{\nu} k^{\alpha}}{\Delta_{0} \Delta_{2} \Delta_{3} \Delta_{5}}=g_{\perp P}^{\mu \nu \alpha} D_{3 g P}^{0235}+g_{\perp R}^{\mu \nu \alpha} D_{3 g R}^{0235}+g_{\perp T}^{\mu \nu \alpha} D_{3 g T}^{0235} \\
& +\{P P P\}^{\mu \nu \alpha} D_{3 P P P}^{0235}+\{R R R\}^{\mu \nu \alpha} D_{3 R R R}^{0235}+\{T T T\}^{\mu \nu \alpha} D_{3 T T T}^{0235} \\
& +\{P P R\}^{\mu \nu \alpha} D_{3 P P R}^{0235}+\{P P T\}^{\mu \nu \alpha} D_{3 P P T}^{0235}+\{R R P\}^{\mu \nu \alpha} D_{3 R R P}^{0126} \\
& +\{R R T\}^{\mu \nu \alpha} D_{3 R R T}^{0235}+\{T T P\}^{\mu \nu \alpha} D_{3 T T P}^{0235}+\{T T R\}^{\mu \nu \alpha} D_{3 T T R}^{0235} \\
& +\{P R T\}^{\mu \nu \alpha} D_{3 P R T}^{0235}
\end{aligned}
$$

where

$$
\begin{aligned}
g_{\perp A}^{\mu \nu \alpha} & =g_{\perp}^{\mu \nu} A^{\alpha}+g_{\perp}^{\mu \alpha} A^{\nu}+g_{\perp}^{\nu \alpha} A^{\mu} \\
\{A A A\}^{\mu \nu \alpha} & =A^{\mu} A^{\nu} A^{\alpha} \\
\{A A B\}^{\mu \nu \alpha} & =A^{\mu} A^{\nu} B^{\alpha}+A^{\mu} A^{\alpha} B^{\nu}+A^{\nu} A^{\alpha} B^{\mu}, \\
\{A B C\}^{\mu \nu \alpha} & =A^{\mu} B^{\nu} C^{\alpha}+A^{\mu} B^{\alpha} C^{\nu}+A^{\nu} B^{\mu} C^{\alpha}+A^{\nu} B^{\alpha} C^{\mu}+A^{\alpha} B^{\mu} C^{\nu}+A^{\alpha} B^{\nu} C^{\mu} .
\end{aligned}
$$

As before the scalar functions defining expansion of rank-3 box integrals are simply fixed by contraction of the integral $D_{3}^{\mu \nu \alpha}$ with corresponding Lorentz structure:

$$
\begin{aligned}
D_{3 A A A} & =\frac{A_{\mu} A_{\nu} A_{\alpha}}{A^{6}} D_{3}^{\mu \nu \alpha}, \\
D_{3 A A B} & =\frac{A_{\mu} A_{\nu} B_{\alpha}}{A^{4} B^{2}} D_{3}^{\mu \nu \alpha}, \\
D_{3 A B C} & =\frac{A_{\mu} B_{\nu} C_{\alpha}}{A^{2} B^{2} C^{2}} D_{3}^{\mu \nu \alpha} \\
D_{3 g A} & =\frac{g_{\mu \nu}^{\perp} A_{\alpha}}{(D-3) A^{2}} D_{3}^{\mu \nu \alpha},
\end{aligned}
$$

where $A, B, C=P, R, T$. We list all scalar functions occurring in the expansion of tensorial rank-2 and rank-3 box integrals in appendix B.3.

Extension on tensorial box integral with higher rank is straightforward. One should just specify the Lorentz structure of corresponding integral and then fix the scalar functions on which the integral is expanded. In particular, decomposition of the rank-4 box inegral with denominators $\Delta_{0}, \Delta_{1}, \Delta_{2}$, and $\Delta_{5}$ reads:

$$
\begin{aligned}
& D_{4}^{\mu \nu \alpha \beta}\left(s, u, Q^{2}\right) \\
&=\left(i \pi^{2}\right)^{-1} \mu^{4-D} \int d^{D} k \frac{k^{\mu} k^{\nu} k^{\alpha} k^{\beta}}{\Delta_{0} \Delta_{1} \Delta_{2} \Delta_{5}} \\
&= g_{\perp}^{\mu \nu \alpha \beta} D_{4 g g}^{0125}+g_{\perp ; P P}^{\mu \nu \alpha \beta} D_{4 g ; P P}^{0125}+g_{\perp ; R R}^{\mu \nu \alpha \beta} D_{4 g ; R R}^{0125}+g_{\perp ; T T}^{\mu \nu \alpha \beta} D_{4 g ; T T}^{0125} \\
&+g_{\perp ; P R}^{\mu \nu \alpha \beta} D_{4 g ; P R}^{0125}+g_{\perp ; P T}^{\mu \nu \alpha \beta} D_{4 g ; P T}^{0125}+g_{\perp ; R T}^{\mu \nu \alpha \beta} D_{4 g ; R T}^{0125} \\
&+\{P P P P\}^{\mu \nu \alpha \beta} D_{4 P P P P}^{0125}+\{R R R R\}^{\mu \nu \alpha \beta} D_{4 R R R R}^{0125}+\{T T T T\}^{\mu \nu \alpha \beta} D_{4 T T T T}^{0125} \\
&+\{P P P R\}^{\mu \nu \alpha \beta} D_{4 P P P R}^{0125}+\{P P P T\}^{\mu \nu \alpha \beta} D_{4 P P P T}^{0125}+\{R R R P\}^{\mu \nu \alpha \beta} D_{4 R R R P}^{0125} \\
&+\{R R R T\}^{\mu \nu \alpha \beta} D_{4 R R R T}^{0125}+\{T T T P\}^{\mu \nu \alpha \beta} D_{4 T T T P}^{0125}+\{T T T R\}^{\mu \nu \alpha \beta} D_{4 T T T R}^{0125} \\
&+\{P P R R\}^{\mu \nu \alpha \beta} D_{4 P P R R}^{0125}+\{P P T T\}^{\mu \nu \alpha \beta} D_{4 P P T T}^{0125}+\{P P R T\}^{\mu \nu \alpha \beta} D_{4 P P R T}^{0125} \\
&+\{R T\}^{\mu 125} \\
&+\{R R T T\}^{\mu \nu \alpha \beta} D_{4 R R T T}^{0125}+\{R R P T\}^{\mu \nu \alpha \beta} D_{4 R R P T}^{0125}+\{T T P R\}^{\mu \nu \alpha \beta} D_{4 T T P R}^{0125},
\end{aligned}
$$


where

$$
\begin{aligned}
g_{\perp}^{\mu \nu \alpha \beta}= & g_{\perp}^{\mu \nu} g_{\perp}^{\alpha \beta}+g_{\perp}^{\mu \alpha} g_{\perp}^{\nu \beta}+g_{\perp}^{\mu \beta} g_{\perp}^{\nu \alpha} \\
g_{\perp ; A A}^{\mu \nu \alpha \beta}= & g_{\perp}^{\mu \nu}\{A A\}^{\alpha \beta}+g_{\perp}^{\mu \alpha}\{A A\}^{\nu \beta}+g_{\perp}^{\mu \beta}\{A A\}^{\nu \alpha}, \\
g_{\perp ; A B}^{\mu \nu \alpha \beta}= & g_{\perp}^{\mu \nu}\{A B\}^{\alpha \beta}+g_{\perp}^{\mu \alpha}\{A B\}^{\nu \beta}+g_{\perp}^{\mu \beta}\{A B\}^{\nu \alpha} \\
& +g_{\perp}^{\alpha \beta}\{A B\}^{\mu \nu}+g_{\perp}^{\nu \beta}\{A B\}^{\mu \alpha}+g_{\perp}^{\nu \alpha}\{A B\}^{\nu \beta} \\
\{A A A A\}^{\mu \nu \alpha \beta}= & A^{\mu} A^{\nu} A^{\alpha} A^{\beta} \\
\{A A A B\}^{\mu \nu \alpha}= & A^{\mu} A^{\nu} A^{\alpha} B^{\beta}+A^{\nu} A^{\alpha} A^{\beta} B^{\mu}+A^{\alpha} A^{\beta} A^{\mu} B^{\nu}+A^{\beta} A^{\mu} A^{\nu} B^{\alpha} \\
\{A A B C\}^{\mu \nu \alpha}= & A^{\mu} A^{\nu} B^{\alpha} C^{\beta}+A^{\mu} A^{\nu} B^{\beta} C^{\alpha}+A^{\nu} A^{\alpha} B^{\beta} C^{\mu}+A^{\nu} A^{\alpha} B^{\mu} C^{\beta} \\
& +A^{\alpha} A^{\beta} B^{\mu} C^{\nu}+A^{\alpha} A^{\beta} B^{\nu} C^{\mu} .
\end{aligned}
$$

Again, all scalar functions defining expansion of rank-4 box integrals are simply fixed by contraction of the integral $D_{4}^{\mu \nu \alpha \beta}$ with corresponding Lorentz structure:

$$
\begin{aligned}
D_{4 g g} & =\frac{1}{(D-3)(D-1)} g_{\mu \nu}^{\perp} g_{\alpha \beta}^{\perp} \cdot D_{4}^{\mu \nu \alpha \beta}, \\
D_{4 g g ; A A} & =\frac{1}{(D-3) A^{4}} g_{\mu \nu}^{\perp} A_{\alpha} A_{\beta} \cdot D_{4}^{\mu \nu \alpha \beta}, \\
D_{4 g g ; A B} & =\frac{1}{(D-3) A^{2} B^{2}} g_{\mu \nu}^{\perp} A_{\alpha} B_{\beta} \cdot D_{4}^{\mu \nu \alpha \beta}, \\
D_{4 A A A A} & =\frac{A_{\mu} A_{\nu} A_{\alpha} A^{\beta}}{A^{8}} D_{4}^{\mu \nu \alpha \beta}, \\
D_{4 A A A B} & =\frac{A_{\mu} A_{\nu} A_{\alpha} B^{\beta}}{A^{6} B^{2}} D_{4}^{\mu \nu \alpha \beta}, \\
D_{4 A A B C} & =\frac{A_{\mu} A_{\nu} B_{\alpha} C^{\beta}}{A^{4} B^{2} C^{2}} D_{4}^{\mu \nu \alpha \beta} .
\end{aligned}
$$

\section{Angular integrals in $D$ dimensions}

In this section we discuss new ideas concerning angular integration in QCD. We extend the known closed results for massless and single massive two denominator integrals in $D$ dimensions derived in refs. $[44,48,65]$ to the double massive case. Furthermore we investigate novel analytic and algebraic properties of angular integrals. An all-order $\varepsilon$ expansion is given for all angular integrals with up to two denominators. A supplementary geometric picture for partial fractioning is developed in appendix D which provides a new rotational invariant algorithm to reduce the number of denominators and also proves to be useful for the double massive integral.

\subsection{Generalized two particle phase space in $D$ dimensions}

To set the stage, we consider the Lorentz invariant phase space integral (PSI)

$$
\int \mathrm{dPS}_{2, P}=\int \frac{\mathrm{d}^{D-1} k_{1}}{(2 \pi)^{D-1} 2 k_{1}^{0}} \int \frac{\mathrm{d}^{D-1} k_{2}}{(2 \pi)^{D-1} 2 k_{2}^{0}}(2 \pi)^{D} \delta^{D}\left(P-k_{1}-k_{2}\right),
$$


where $P=p_{1}+p_{2}+\cdots+p_{n}$ is the sum of some fixed momenta. One might think of this PSI potentially as a part of some larger PSI. $P$ shall be time-like and future-pointing such that there exists a rest frame of $P$ with $P^{0}>0$. The $k_{1}$ and $k_{2}$ are assumed as momenta of massless particles, i.e. $k_{1}^{0}=\left|\mathbf{k}_{\mathbf{1}}\right|$ and $k_{2}^{0}=\left|\mathbf{k}_{\mathbf{2}}\right|$. But we should stress that, in general, final particles are not neccessary massless and we can deal with massive particles (see also ref. [49]).

Applying the identity

$$
\int \mathrm{d}^{D} k \delta\left(k^{2}\right) \theta\left(E_{k}\right) f(k)=\int \frac{\mathrm{d}^{D-1} k}{2 k^{0}} f(k),
$$

for the integral over $k_{2}$ and evaluating it using the $D$ dimensional $\delta$-function, the phase space is written as

$$
\int \mathrm{dPS}_{2, P}=\int \frac{\mathrm{d}^{D-1} k_{1}}{(2 \pi)^{D-2}\left|\mathbf{k}_{\mathbf{1}}\right|} \delta\left(\left(P_{1}-k_{1}\right)^{2}\right) \theta\left(P^{0}-k_{1}^{0}\right) .
$$

By choosing the rest frame of $P$ we have $P=\left(\sqrt{P^{2}}, \mathbf{0}\right)$ and $k_{1}=(|\mathbf{k}|, \mathbf{k})$ leading to $(P-$ $\left.k_{1}\right)^{2}=2 \sqrt{P^{2}}\left(\frac{\sqrt{P^{2}}}{2}-|\mathbf{k}|\right)$. Employing spherical coordinates in this frame and evaluating the radial integral, we get

$$
\begin{aligned}
\int \mathrm{dPS}_{2, P} & =\frac{1}{2(2 \pi)^{D-2} \sqrt{P^{2}}} \int_{0}^{\infty} \mathrm{d}|\mathbf{k}||\mathbf{k}|^{D-3} \int \mathrm{d} \Omega_{D-2} \delta\left(|\mathbf{k}|-\frac{\sqrt{P^{2}}}{2}\right) \theta\left(\sqrt{P^{2}}-|\mathbf{k}|\right) \\
& =\frac{\left(P^{2}\right)^{\frac{D-4}{2}}}{2(4 \pi)^{D-2}} \int \mathrm{d} \Omega_{D-2} .
\end{aligned}
$$

Notice that the $\theta$-function is trivially satisfied since $P^{0}>0$. If we are in a situation, where the function we want to integrate over the phase space, does only depend on two angles, the remaining $D-4$ dimensional spherical integral can be integrated out (see details of spherical integration in dimensional regularization in appendix C). Defining

$$
\mathrm{d} \Omega_{k_{1} k_{2}} \equiv \mathrm{d} \theta_{1} \sin ^{1-2 \varepsilon} \theta_{1} \mathrm{~d} \theta_{2} \sin ^{-2 \varepsilon} \theta_{2}
$$

with $D=4-2 \varepsilon$ and using the area formula for $n$ dimensional spheres

$$
\Omega_{n}=\frac{2 \pi^{\frac{n+1}{2}}}{\Gamma\left(\frac{n+1}{2}\right)}=\frac{2^{n} \pi^{\frac{n}{2}} \Gamma\left(\frac{n}{2}\right)}{\Gamma(n)}=\frac{2(4 \pi)^{\frac{n}{2}} \Gamma\left(1+\frac{n}{2}\right)}{\Gamma(1+n)}
$$

the angular integral becomes

$$
\int \mathrm{d} \Omega_{D-2}=\int \mathrm{d} \Omega_{D-4} \int \mathrm{d} \Omega_{k_{1} k_{2}} \longrightarrow \Omega_{D-4} \int \mathrm{d} \Omega_{k_{1} k_{2}}=\frac{2(4 \pi)^{-\varepsilon} \Gamma(1-\varepsilon)}{\Gamma(1-2 \varepsilon)} \int \mathrm{d} \Omega_{k_{1} k_{2}} .
$$

Therefore, we find the well known formula for the two-body phase space in $D=4-2 \varepsilon$ dimensions in the rest frame of $P$

$$
\int \mathrm{dPS}_{2, P}=\frac{\Gamma(1-\varepsilon)}{(4 \pi)^{2-\varepsilon} \Gamma(1-2 \varepsilon)}\left(P^{2}\right)^{-\varepsilon} \int \mathrm{d} \Omega_{k_{1} k_{2}} .
$$

If the integrand of the PSI is a rational function of the angular dependent Mandelstam variables, then it can be further simplified and reduced to a set of master integrals by partial fraction decomposition. In appendix D we discuss in details a novel geometric approach developed for this task. There, we considered the double real corrections in Drell-Yan process as an illustration of how the partial fractioning works (see appendix D.6). 


\subsection{Analytic evaluation of angular integrals in $D$ dimension}

We introduce a set of basis integrals to which all of the Neerven integrals can be reduced, but that are characterized by properties that are invariant under rotation rather than the parameters $a, b, A, B$, and $C$ from eq. (1.1). They will now depend on the variables $v_{i j}=v_{i} \cdot v_{j}$, which are all invariant under change of coordinates. ${ }^{1}$ We define

Zero-denominator integral:

$$
I^{(0)}(\varepsilon):=\int \mathrm{d} \Omega_{k_{1} k_{2}} .
$$

One-denominator massless integral:

$$
I_{j}^{(0)}(\varepsilon):=\int \mathrm{d} \Omega_{k_{1} k_{2}} \frac{1}{\left(v_{1} \cdot k\right)^{j}}, \quad v_{11}=0 .
$$

One-denominator massive integral:

$$
I_{j}^{(1)}\left(v_{11} ; \varepsilon\right):=\int \mathrm{d} \Omega_{k_{1} k_{2}} \frac{1}{\left(v_{1} \cdot k\right)^{j}}, \quad \quad v_{11} \neq 0 .
$$

Two-denominator massless integral:

$$
I_{j, l}^{(0)}\left(v_{12} ; \varepsilon\right):=\int \mathrm{d} \Omega_{k_{1} k_{2}} \frac{1}{\left(v_{1} \cdot k\right)^{j}\left(v_{2} \cdot k\right)^{l}}, \quad v_{11}=v_{22}=0 .
$$

Two-denominator single-massive integral:

$$
I_{j, l}^{(1)}\left(v_{12}, v_{11} ; \varepsilon\right):=\int \mathrm{d} \Omega_{k_{1} k_{2}} \frac{1}{\left(v_{1} \cdot k\right)^{j}\left(v_{2} \cdot k\right)^{l}}, \quad v_{11} \neq 0, v_{22}=0 .
$$

Two-denominator double-massive integral:

$$
I_{j, l}^{(2)}\left(v_{12}, v_{11}, v_{22} ; \varepsilon\right):=\int \mathrm{d} \Omega_{k_{1} k_{2}} \frac{1}{\left(v_{1} \cdot k\right)^{j}\left(v_{2} \cdot k\right)^{l}}, \quad v_{11} \neq 0, v_{22} \neq 0 .
$$

Here

$$
\int \mathrm{d} \Omega_{k_{1} k_{2}}=\int_{0}^{\pi} \mathrm{d} \theta_{1} \sin ^{1-2 \varepsilon} \theta_{1} \int_{0}^{\pi} \mathrm{d} \theta_{2} \sin ^{-2 \varepsilon} \theta_{2},
$$

is the integration measure in a frame with $k=\left(1, \ldots, \cos \theta_{2} \sin \theta_{1}, \cos \theta_{1}\right)$ [the dots represent irrelevant angles since $\mathbf{v}_{\mathbf{1}}$ and $\mathbf{v}_{\mathbf{2}}$ lie in the $\left(x_{D-1}, x_{D}\right)$-plane]. The upper number in parenthesis gives the number of masses involved and the number of lower indices gives the number of different denominators. All appearing $D$-vectors are normalized such that their 0 -component is equal to 1 .

We will calculate the integrals one by one which has the benefit that we gradually increase the difficulty of the calculation and can introduce the employed methods one by one.

\footnotetext{
${ }^{1}$ Note that this more natural normalization is different than that used by Somogyi [48], which is taylored to the Mellin-Barnes representations.
} 


\subsubsection{Integral without denominator}

We start with the basic integral $I^{(0)}(\varepsilon)$ without any denominator

$$
I^{(0)}(\varepsilon)=\int \mathrm{d} \Omega_{k_{1} k_{2}}=\int_{0}^{\pi} \mathrm{d} \theta_{1} \sin ^{1-2 \varepsilon} \theta_{1} \int_{0}^{\pi} \mathrm{d} \theta_{2} \sin ^{-2 \varepsilon} \theta_{2} .
$$

Changing the variables in both integrals as $\cos \theta_{i}=1-2 t_{i}$ with $d \theta_{i} \sin \theta_{i}=2 d t_{i}$ and $\sin \theta_{i}=2 \sqrt{t_{i}\left(1-t_{i}\right)}$ we rewrite the integral $I^{(0)}(\varepsilon)$ in the form

$$
I^{(0)}(\varepsilon)=2^{1-4 \varepsilon} \int_{0}^{1} \mathrm{~d} t_{1} t_{1}^{-\varepsilon}\left(1-t_{1}\right)^{-\varepsilon} \int_{0}^{1} \mathrm{~d} t_{2} t_{2}^{-\frac{1}{2}-\varepsilon}\left(1-t_{2}\right)^{-\frac{1}{2}-\varepsilon},
$$

containing the product of two integral representations of the beta function

$$
B(x, y)=\frac{\Gamma(x) \Gamma(y)}{\Gamma(x+y)}=\int_{0}^{1} \mathrm{~d} t t^{x-1}(1-t)^{y-1} .
$$

Taking into account eq. (3.18) we finally get:

$$
I^{(0)}(\varepsilon)=2^{1-4 \varepsilon} \frac{\Gamma^{2}(1-\varepsilon)}{\Gamma(2-2 \varepsilon)} \frac{\Gamma^{2}\left(\frac{1}{2}-\varepsilon\right)}{\Gamma(1-2 \varepsilon)} .
$$

Using the Legendre duplication formula

$$
\Gamma\left(\frac{1}{2}-\varepsilon\right) \Gamma(1-\varepsilon)=\frac{\sqrt{\pi}}{2^{-2 \varepsilon}} \Gamma(1-2 \varepsilon),
$$

$I^{(0)}(\varepsilon)$ is further simplified to

$$
I^{(0)}(\varepsilon)=2 \pi \frac{\Gamma(1-2 \varepsilon)}{\Gamma(2-2 \varepsilon)}=\frac{2 \pi}{1-2 \varepsilon} .
$$

The latter factor will occur as universal multiplier in all other angular integrals.

\subsubsection{Massless integral with one denominator}

In the calculations of the angular integrals we follow ideas proposed and developed in ref. [48]. In particular, for the calculation of the massless one denominator integral $I_{j}^{(0)}(\varepsilon)$ we rotate the frame such that the unit vector $\mathbf{v}_{\mathbf{1}}$ points in the $x_{D}$-direction, i.e. $v_{1}=$ $\left(1, \mathbf{0}_{D-3}, 0,1\right), k=\left(1, \mathbf{0}_{D-3}, \sin \theta_{1} \cos \theta_{2}, \cos \theta_{1}\right)$, and $v_{1} \cdot k=1-\cos \theta_{1}$. Therefore, we have

$$
\begin{aligned}
I_{j}^{(0)}(\varepsilon) & =\int \mathrm{d} \Omega_{k_{1} k_{2}} \frac{1}{\left(v_{1} \cdot k\right)^{j}} \\
& =\int_{0}^{\pi} \mathrm{d} \theta_{1} \frac{\sin ^{1-2 \varepsilon} \theta_{1}}{\left(1-\cos \theta_{1}\right)^{j}} \int_{0}^{\pi} \mathrm{d} \theta_{2} \sin ^{-2 \varepsilon} \theta_{2} .
\end{aligned}
$$

Next we proceed by analogy with the integral $I^{(0)}(\varepsilon)$ : employing the same change of variables and using the integral representation (3.18) for the beta function. We find:

$$
\begin{aligned}
I_{j}^{(0)}(\varepsilon) & =2^{1-j-4 \varepsilon} \int_{0}^{1} \mathrm{~d} t_{1} t_{1}^{-j-\varepsilon}\left(1-t_{1}\right)^{-\varepsilon} \int_{0}^{1} \mathrm{~d} t_{2} t_{2}^{-\frac{1}{2}-\varepsilon}\left(1-t_{2}\right)^{-\frac{1}{2}-\varepsilon} \\
& =2^{1-j-4 \varepsilon} \frac{\Gamma(1-j-\varepsilon) \Gamma(1-\varepsilon)}{\Gamma(2-j-2 \varepsilon)} \frac{\Gamma^{2}\left(\frac{1}{2}-\varepsilon\right)}{\Gamma(1-2 \varepsilon)} .
\end{aligned}
$$


Separating the factor $I^{(0)}(\varepsilon)$ and using the Pochhammer symbol

$$
(x)_{n}=\frac{\Gamma(x+n)}{\Gamma(x)}
$$

yields

$$
I_{j}^{(0)}(\varepsilon)=\frac{2 \pi}{1-2 \varepsilon} 2^{-j} \frac{\Gamma(1-j-\varepsilon) \Gamma(2-2 \varepsilon)}{\Gamma(1-\varepsilon) \Gamma(2-j-2 \varepsilon)}=I^{(0)}(\varepsilon) \frac{(2-j-2 \varepsilon)_{j}}{2^{j}(1-j-\varepsilon)_{j}} .
$$

In this form $I_{j}^{(0)}(\varepsilon)$ is a manifestly rational function of $\varepsilon$ if $j \in \mathbb{Z}$, such that apparently no polygamma constants will clutter the $\varepsilon$-expansion.

\subsubsection{Massive integral with one denominator}

We choose the same frame as in the massless case with $\mathbf{v}_{1}$ in $x_{D}$ direction: $v=\left(1, \mathbf{0}_{D-3}, 0, \beta\right)$ and $v_{1} \cdot k=1-\beta \cos \theta_{1}$ with $v_{11} \neq 0$ and $\beta \equiv \sqrt{1-v_{11}} \neq 1$. Therefore,

$$
\begin{aligned}
I_{j}^{(1)}\left(v_{11} ; \varepsilon\right) & =\int \mathrm{d} \Omega_{k_{1} k_{2}} \frac{1}{\left(v_{1} \cdot k\right)^{j}} \\
& =\int_{0}^{\pi} \mathrm{d} \theta_{1} \frac{\sin ^{1-2 \varepsilon} \theta_{1}}{\left(1-\beta \cos \theta_{1}\right)^{j}} \int_{0}^{\pi} \mathrm{d} \theta_{2} \sin ^{-2 \varepsilon} \theta_{2} .
\end{aligned}
$$

The $\cos \theta_{1}=1-2 t$ substitution now gives

$$
1-\beta \cos \theta_{1}=(1-\beta)\left(1+\frac{2 \beta}{1-\beta} t\right)
$$

thus

$$
I_{j}^{(1)}\left(v_{11} ; \varepsilon\right)=\frac{2^{1-4 \varepsilon}}{(1-\beta)^{j}} \int_{0}^{1} \mathrm{~d} t_{1} t_{1}^{-\varepsilon}\left(1-t_{1}\right)^{-\varepsilon}\left(1+\frac{2 \beta}{1-\beta} t\right)^{-j} \int_{0}^{1} \mathrm{~d} t_{2} t_{2}^{-\frac{1}{2}-\varepsilon}\left(1-t_{2}\right)^{-\frac{1}{2}-\varepsilon} .
$$

Next, using the integral representation for the Gauss hypergeometric function (Euler formula)

$$
{ }_{2} F_{1}(a, b, c, z)=\frac{\Gamma(c)}{\Gamma(b) \Gamma(c-b)} \int_{0}^{1} \mathrm{~d} t t^{b-1}(1-t)^{c-b-1}(1-z t)^{-a},
$$

in case of the integral over $t_{1}$ and isolating the factor $I^{(0)}(\varepsilon)$ one gets:

$$
\begin{aligned}
I_{j}^{(1)}\left(v_{11} ; \varepsilon\right) & =\frac{2^{1-4 \varepsilon}}{(1-\beta)^{j}} \frac{\Gamma^{2}(1-\varepsilon)}{\Gamma(2-2 \varepsilon)}{ }_{2} F_{1}\left(j, 1-\varepsilon, 2-2 \varepsilon,-\frac{2 \beta}{1-\beta}\right) \frac{\Gamma^{2}\left(\frac{1}{2}-\varepsilon\right)}{\Gamma(1-2 \varepsilon)} \\
& =\frac{I^{(0)}(\varepsilon)}{(1-\beta)^{j}}{ }_{2} F_{1}\left(j, 1-\varepsilon, 2-2 \varepsilon,-\frac{2 \beta}{1-\beta}\right) .
\end{aligned}
$$

Here we should make two important remarks on the Gauss function: (1) the Gauss function with integer coefficients occurring in eq. (3.30) is well suited for the $\varepsilon$-expansion; (2) the Gauss function can be defined by the power series using Pochhammer symbol as:

$$
{ }_{2} F_{1}(a, b, c, z)=\sum_{n=0}^{\infty} \frac{(a)_{n}(b)_{n}}{(c)_{n}} \frac{z^{n}}{n !},
$$

which is manifestly symmetric under interchange of the first and second arguments $a \leftrightarrow b$. 
The integral $I_{j}^{(1)}\left(v_{11} ; \varepsilon\right)$ will be a cornerstone for consideration of further integrals. For this purpose it is feasible to deduce a Mellin-Barnes representation of the integral, which has a particularly simple dependence on $v_{11}$. To this end we first employ the quadratic hypergeometric transformation

$$
{ }_{2} F_{1}(a, b, 2 b, x)=\left(1-\frac{x}{2}\right)^{-a}{ }_{2} F_{1}\left(\frac{a}{2}, \frac{a+1}{2}, b+\frac{1}{2},\left(\frac{x}{2-x}\right)^{2}\right),
$$

to convert $I_{j}^{(1)}\left(v_{11} ; \varepsilon\right)$ into

$$
I_{j}^{(1)}\left(v_{11} ; \varepsilon\right)=I^{(0)}(\varepsilon){ }_{2} F_{1}\left(\frac{j}{2}, \frac{j+1}{2}, \frac{3}{2}-\varepsilon, 1-v_{11}\right) .
$$

In a second step we use the Mellin-Barnes representation

$$
\begin{aligned}
{ }_{2} F_{1}(a, b, c, x)= & \frac{\Gamma(c)}{\Gamma(a) \Gamma(b) \Gamma(c-a) \Gamma(c-b)} \\
& \times \int_{-i \infty}^{i \infty} \frac{\mathrm{d} z}{2 \pi i} \Gamma(a+z) \Gamma(b+z) \Gamma(c-a-b-z) \Gamma(-z)(1-x)^{z} .
\end{aligned}
$$

This results in

$$
\begin{aligned}
I_{j}^{(1)}\left(v_{11} ; \varepsilon\right)= & I^{(0)}(\varepsilon) \frac{\Gamma\left(\frac{3}{2}-\varepsilon\right)}{\Gamma\left(\frac{j}{2}\right) \Gamma\left(\frac{j+1}{2}\right) \Gamma\left(\frac{3-j}{2}-\varepsilon\right) \Gamma\left(\frac{2-j}{2}-\varepsilon\right)} \\
& \times \int_{-i \infty}^{i \infty} \frac{\mathrm{d} z}{2 \pi i} \Gamma\left(\frac{j}{2}+z\right) \Gamma\left(\frac{j+1}{2}+z\right) \Gamma(1-j-\varepsilon-z) \Gamma(-z) v_{11}^{z} .
\end{aligned}
$$

Applying the Legendre duplication formula on each gamma function that contains a half integer, results in the Mellin-Barnes representation

$$
I_{j}^{(1)}\left(v_{11} ; \varepsilon\right)=\frac{I^{(0)}(\varepsilon)}{\Gamma(1-\varepsilon)} \frac{(2-j-2 \varepsilon)_{j}}{2^{j} \Gamma(j)} \int_{-i \infty}^{i \infty} \frac{\mathrm{d} z}{2 \pi i} \Gamma(j+2 z) \Gamma(1-j-\varepsilon-z) \Gamma(-z)\left(\frac{v_{11}}{4}\right)^{z} .
$$

This form will prove valuable for further calculations due to its simple dependence on $v_{11}$.

As a crosscheck of our calculations we are able to demonstrate that applying the limit $v_{11} \rightarrow 0$ we get $I_{j}^{(1)}\left(v_{11}, \varepsilon\right) \stackrel{v_{11} \rightarrow 0}{\longrightarrow} I_{j}^{(0)}(\varepsilon)$. For this purpose the form (3.33) is well suited. In particular, using the Gauss theorem

$$
{ }_{2} F_{1}(a, b, c, 1)=\frac{\Gamma(c) \Gamma(c-a-b)}{\Gamma(c-a) \Gamma(c-b)} \text { for } \operatorname{Re}(c)>\operatorname{Re}(a+b),
$$

and the Legendre duplication formula one gets

$$
\begin{aligned}
I_{j}^{(1)}(0, \varepsilon) & =I^{(0)}(\varepsilon)_{2} F_{1}\left(\frac{j}{2}, \frac{j+1}{2}, \frac{3}{2}-\varepsilon, 1\right) \\
& =I^{(0)}(\varepsilon) \frac{\Gamma\left(\frac{3}{2}-\varepsilon\right) \Gamma(1-j-\varepsilon)}{\Gamma\left(\frac{3-j}{2}-\varepsilon\right) \Gamma\left(1-\frac{j}{2}-\varepsilon\right)} \\
& =I^{(0)}(\varepsilon) \frac{(2-j-2 \varepsilon)_{j}}{2^{j}(1-j-\varepsilon)_{j}}=I_{j}^{(0)}(\varepsilon) .
\end{aligned}
$$




\subsubsection{Massless integral with two denominators}

For the two denominator case there is the issue that the $\theta_{1}$ and $\theta_{2}$ integrals do no longer factorize. Therefore, it is feasible to disregard a direct $t$-substitution and favor an approach based on Feynman parametrization to combine the denominators. For the moment we assume $j, l \notin \mathbb{Z}_{\leq 0}$. Taking advantage of the linearity of the propagator this results in

$$
\begin{aligned}
I_{j, l}^{(0)}\left(v_{12} ; \varepsilon\right)= & \int \mathrm{d} \Omega_{k_{1} k_{2}} \frac{1}{\left(v_{1} \cdot k\right)^{j}\left(v_{2} \cdot k\right)^{l}} \\
= & \frac{1}{B(j, l)} \int_{0}^{1} \mathrm{~d} x_{1} x_{1}^{j-1} \int_{0}^{1} \mathrm{~d} x_{2} x_{2}^{l-1} \delta\left(1-x_{1}-x_{2}\right) \\
& \times \int \mathrm{d} \Omega_{k_{1} k_{2}} \frac{1}{\left(\left(x_{1} v_{1}+x_{2} v_{2}\right) \cdot k\right)^{j+l}} .
\end{aligned}
$$

We introduce the vector $v \equiv x_{1} v_{1}+x_{2} v_{2}$ and rotate the coordinate system such that $\mathbf{v}$ points in the $x_{D}$-direction. In this frame it holds $v=\left(1, \mathbf{0}_{D-3}, 0, \sqrt{1-2 x_{1} x_{2} v_{12}}\right)$, since in the massless case we have $v_{11}=v_{22}=0$ such that $v^{2}=\left(x_{1} v_{1}+x_{2} v_{2}\right)^{2}=2 x_{1} x_{2} v_{12}$. Thus, we can write

$$
I_{j, l}^{(0)}\left(v_{12} ; \varepsilon\right)=\frac{\Gamma(j+l)}{\Gamma(j) \Gamma(l)} \int_{0}^{1} \mathrm{~d} x_{1} x_{1}^{j-1} \int_{0}^{1} \mathrm{~d} x_{2} x_{2}^{l-1} \delta\left(1-x_{1}-x_{2}\right) I_{j+l}^{(1)}\left(2 x_{1} x_{2} v_{12}\right) .
$$

Employing the Mellin-Barnes representation of $I_{j}^{(1)}\left(v_{11} ; \varepsilon\right)$ from eq. (3.36) brings this into the form

$$
\begin{aligned}
I_{j, l}^{(0)}\left(v_{12} ; \varepsilon\right)= & \frac{I^{(0)}(\varepsilon)}{\Gamma(1-\varepsilon)} \frac{(2-j-l-2 \varepsilon)_{j+l}}{2^{j+l} \Gamma(j) \Gamma(l)} \int_{0}^{1} \mathrm{~d} x_{1} x_{1}^{j-1} \int_{0}^{1} \mathrm{~d} x_{2} x_{2}^{l-1} \delta\left(1-x_{1}-x_{2}\right) \\
& \times \int_{-i \infty}^{i \infty} \frac{\mathrm{d} z}{2 \pi i} \Gamma(j+l+2 z) \Gamma(1-j-l-\varepsilon-z) \Gamma(-z)\left(\frac{x_{1} x_{2} v_{12}}{2}\right)^{z} .
\end{aligned}
$$

Now the Feynman parameter integrals are factorized and can be evaluated in terms of beta function as

$$
\int_{0}^{1} \mathrm{~d} x_{1} x_{1}^{j+z-1} \int_{0}^{1} \mathrm{~d} x_{2} x_{2}^{l+z-1} \delta\left(1-x_{1}-x_{2}\right)=B(j+z, l+z) .
$$

Using the Mellin-Barnes representation for the Gauss hypergeometric function (3.34) and eq. (3.42) we get

$$
\begin{aligned}
I_{j, l}^{(0)}\left(v_{12} ; \varepsilon\right)= & \frac{I^{(0)}(\varepsilon)}{\Gamma(1-\varepsilon)} \frac{(2-j-l-2 \varepsilon)_{j+l}}{2^{j+l} \Gamma(j) \Gamma(l)} \\
& \times \int_{-i \infty}^{i \infty} \frac{\mathrm{d} z}{2 \pi i} \Gamma(j+z) \Gamma(l+z) \Gamma(1-j-l-\varepsilon-z) \Gamma(-z)\left(\frac{v_{12}}{2}\right)^{z} \\
= & I^{(0)}(\varepsilon) \frac{(2-j-l-2 \varepsilon)_{j+l}}{2^{j+l}} \frac{\Gamma(1-j-\varepsilon) \Gamma(1-l-\varepsilon)}{\Gamma^{2}(1-\varepsilon)}{ }_{2} F_{1}\left(j, l, 1-\varepsilon, 1-\frac{v_{12}}{2}\right) \\
= & I^{(0)}(\varepsilon) \frac{(2-j-l-2 \varepsilon)_{j+l}}{2^{j+l}(1-j-\varepsilon)_{j}(1-l-\varepsilon)_{l}}{ }_{2} F_{1}\left(j, l, 1-\varepsilon, 1-\frac{v_{12}}{2}\right),
\end{aligned}
$$


or alternatively by factorizing out the integral $I_{j+l}^{(0)}(\varepsilon)$ :

$$
I_{j, l}^{(0)}\left(v_{12} ; \varepsilon\right)=I_{j+l}^{(0)}(\varepsilon) \frac{(1-j-l-\varepsilon)_{j}}{(1-j-\varepsilon)_{j}}{ }_{2} F_{1}\left(j, l, 1-\varepsilon, 1-\frac{v_{12}}{2}\right) .
$$

This result also holds for the previously excluded cases $j, l \in \mathbb{Z}_{\leq 0}$. As for the integral with one massive denominator we obtain a hypergeometric function to be expanded in $\varepsilon$ about integer parameters.

Using the Gauss theorem (3.37) we obtain in the limit $v_{12} \rightarrow 0$

$$
\begin{aligned}
I_{j, l}^{(0)}(0 ; \varepsilon) & =I_{j+l}^{(0)}(\varepsilon) \frac{(1-j-l-\varepsilon)_{j}}{(1-j-\varepsilon)_{j}}{ }_{2} F_{1}(j, l, 1-\varepsilon, 1) \\
& =I_{j+l}^{(0)}(\varepsilon) \frac{(1-j-l-\varepsilon)_{j}}{(1-j-\varepsilon)_{j}} \frac{\Gamma(1-\varepsilon) \Gamma(1-j-l-\varepsilon)}{\Gamma(1-j-\varepsilon) \Gamma(1-l-\varepsilon)}=I_{j+l}^{(0)}(\varepsilon) .
\end{aligned}
$$

One can see that the expressions (3.43) and (3.44) are manifestly symmetric under interchange $j \leftrightarrow l$, hence $I_{j, l}^{(0)}\left(v_{12} ; \varepsilon\right)=I_{l, j}^{(0)}\left(v_{12} ; \varepsilon\right)$. From eq. (3.44) it is evident that $I_{j, 0}^{(0)}\left(v_{12} ; \varepsilon\right)=I_{j}^{(0)}(\varepsilon)$.

\subsubsection{Single massive integral with two denominators}

Now we consider the single massive case

$$
I_{j, l}^{(1)}\left(v_{12}, v_{11} ; \varepsilon\right)=\int \mathrm{d} \Omega_{k_{1} k_{2}} \frac{1}{\left(v_{1} \cdot k\right)^{j}\left(v_{2} \cdot k\right)^{l}}, \quad v_{11} \neq 0, v_{22}=0 .
$$

We can start our consideration after Feynman parametrization of the two denominator integral. The vector $v$ has now norm $v^{2}=x_{1}^{2} v_{11}+2 x_{1} x_{2} v_{12}$. Employing again the MellinBarnes representation of $I_{j+l}^{(1)}\left(v^{2}\right)$ gives [compare with eq. (3.41)]

$$
\begin{aligned}
I_{j, l}^{(1)}\left(v_{12}, v_{11} ; \varepsilon\right)= & \frac{I^{(0)}(\varepsilon)}{\Gamma(1-\varepsilon)} \frac{(2-j-l-2 \varepsilon)_{j+l}}{2^{j+l} \Gamma(j) \Gamma(l)} \int_{0}^{1} \mathrm{~d} x_{1} x_{1}^{j-1} \int_{0}^{1} \mathrm{~d} x_{2} x_{2}^{l-1} \delta\left(1-x_{1}-x_{2}\right) \\
& \times \int_{-i \infty}^{i \infty} \frac{\mathrm{d} z}{2 \pi i} \Gamma(j+l+2 z) \Gamma(1-j-l-\varepsilon-z) \Gamma(-z)\left(\frac{x_{1}^{2} v_{11}}{4}+\frac{x_{1} x_{2} v_{12}}{2}\right)^{z} .
\end{aligned}
$$

In order to evaluate the Feynman parameter integral following our strategy in section 3.2.4, a factorization of $\left(\frac{x_{1}^{2} v_{11}}{4}+\frac{x_{1} x_{2} v_{12}}{2}\right)^{z}$ is required. Applying the Binomi-Mellin-Newton integral representation ${ }^{2}$

$$
(a+b)^{z_{1}}=\frac{1}{\Gamma\left(-z_{1}\right)} \int_{-i \infty}^{i \infty} \frac{\mathrm{d} z_{2}}{2 \pi i} a^{z_{1}-z_{2}} b^{z_{2}} \Gamma\left(-z_{2}\right) \Gamma\left(-z_{1}+z_{2}\right)
$$

\footnotetext{
${ }^{2}$ This integral representation does not have a name in the literature (see, e.g., eq. (5.1) in ref. [25], where it is just referred to as a simple formula, however due to its frequent occurrence and usefulness, it deserves, at least in the authors' opinion, a name analogous to the well-established Cahen-Mellin integral. As (B.31) is the Mellin integral version of Newton's Binomial Theorem this name suggests itself.
} 
on this term, results in

$$
\begin{aligned}
I_{j, l}^{(1)}\left(v_{12}, v_{11} ; \varepsilon\right)= & \frac{I^{(0)}(\varepsilon)}{\Gamma(1-\varepsilon)} \frac{(2-j-l-2 \varepsilon)_{j+l}}{2^{j+l} \Gamma(j) \Gamma(l)} \int_{-i \infty}^{i \infty} \frac{\mathrm{d} z_{1}}{2 \pi i} \Gamma\left(j+l+2 z_{1}\right) \Gamma\left(1-j-l-\varepsilon-z_{1}\right) \\
& \times \int_{-i \infty}^{i \infty} \frac{\mathrm{d} z_{2}}{2 \pi i}\left(\frac{v_{11}}{4}\right)^{z_{1}-z_{2}}\left(\frac{v_{12}}{2}\right)^{z_{2}} \Gamma\left(-z_{2}\right) \Gamma\left(-z_{1}+z_{2}\right) \\
& \times \int_{0}^{1} \mathrm{~d} x_{1} x_{1}^{j+2 z_{1}-z_{2}-1} \int_{0}^{1} \mathrm{~d} x_{2} x_{2}^{l+z_{2}-1} \delta\left(1-x_{1}-x_{2}\right) .
\end{aligned}
$$

Evaluating the Feynman parameter integral again cancels the $\Gamma\left(j+l+2 z_{1}\right)$ and yields

$$
\begin{aligned}
I_{j, l}^{(1)}\left(v_{12}, v_{11} ; \varepsilon\right)= & \frac{I^{(0)}(\varepsilon)}{\Gamma(1-\varepsilon)} \frac{(2-j-l-2 \varepsilon)_{j+l}}{2^{j+l} \Gamma(j) \Gamma(l)} \int_{-i \infty}^{i \infty} \frac{\mathrm{d} z_{1}}{2 \pi i} \int_{-i \infty}^{i \infty} \frac{\mathrm{d} z_{2}}{2 \pi i}\left(\frac{v_{11}}{4}\right)^{z_{1}}\left(\frac{2 v_{12}}{v_{11}}\right)^{z_{2}} \\
& \times \Gamma\left(1-j-l-\varepsilon-z_{1}\right) \Gamma\left(-z_{1}+z_{2}\right) \Gamma\left(j+2 z_{1}-z_{2}\right) \Gamma\left(-z_{2}\right) \Gamma\left(l+z_{2}\right) .
\end{aligned}
$$

Performing the substitutions $z_{1} \rightarrow 1-j-l-\varepsilon+z_{1}$ and $z_{2} \rightarrow 1-j-l-\varepsilon+z_{1}-z_{2}$ results in the two-fold Mellin-Barnes representation

$$
\begin{aligned}
I_{j, l}^{(1)}\left(v_{12}, v_{11} ; \varepsilon\right)= & \frac{I^{(0)}(\varepsilon)}{\Gamma(1-\varepsilon)} \frac{(2-j-l-2 \varepsilon)_{j+l}}{2^{j+l} \Gamma(j) \Gamma(l)} \int_{-i \infty}^{i \infty} \frac{\mathrm{d} z_{1}}{2 \pi i} \int_{-i \infty}^{i \infty} \frac{\mathrm{d} z_{2}}{2 \pi i}\left(\frac{v_{11}}{4}\right)^{1-j-l-\varepsilon+z_{1}} \\
& \times\left(\frac{2 v_{12}}{v_{11}}\right)^{1-j-l-\varepsilon+z_{1}-z_{2}} \Gamma\left(-z_{1}\right) \Gamma\left(-z_{2}\right) \Gamma\left(1-l-\varepsilon+z_{1}+z_{2}\right) \\
& \times \Gamma\left(-1+j+l+\varepsilon-z_{1}+z_{2}\right) \Gamma\left(1-j-\varepsilon+z_{1}-z_{2}\right) .
\end{aligned}
$$

Replacing the product of the last two gamma functions in eq. (3.51) by the corresponding beta function integral representation

$$
\begin{array}{r}
\Gamma\left(-1+j+l+\varepsilon-z_{1}+z_{2}\right) \Gamma\left(1-j-\varepsilon+z_{1}-z_{2}\right) \\
\quad=\Gamma(l) \int_{0}^{1} \mathrm{~d} t t^{-2+j+l+\varepsilon-z_{1}+z_{2}}(1-t)^{-j-\varepsilon+z_{1}-z_{2}},
\end{array}
$$

leads to

$$
\begin{aligned}
I_{j, l}^{(1)}\left(v_{12}, v_{11} ; \varepsilon\right)= & \frac{I^{(0)}(\varepsilon)}{\Gamma(1-\varepsilon)} \frac{(2-j-l-2 \varepsilon)_{j+l}}{2^{j+l} \Gamma(j)}\left(\frac{v_{12}}{2}\right)^{1-j-l-\varepsilon} \int_{0}^{1} \mathrm{~d} t t^{-2+j+l+\varepsilon}(1-t)^{-j-\varepsilon} \\
& \times \int_{-i \infty}^{i \infty} \frac{\mathrm{d} z_{1}}{2 \pi i} \int_{-i \infty}^{i \infty} \frac{\mathrm{d} z_{2}}{2 \pi i}\left(\frac{1-t}{t} \frac{v_{12}}{2}\right)^{z_{1}}\left(\frac{t}{1-t} \frac{v_{11}}{2 v_{12}}\right)^{z_{2}} \\
& \times \Gamma\left(-z_{1}\right) \Gamma\left(-z_{2}\right) \Gamma\left(1-l-\varepsilon+z_{1}+z_{2}\right) .
\end{aligned}
$$

The appearing two-fold Mellin-Barnes integral evaluates by the Binomi-Mellin-Newton integral to

$$
\begin{aligned}
& \int_{-i \infty}^{i \infty} \frac{\mathrm{d} z_{1}}{2 \pi i} \int_{-i \infty}^{i \infty} \frac{\mathrm{d} z_{2}}{2 \pi i}\left(\frac{1-t}{t} \frac{v_{12}}{2}\right)^{z_{1}}\left(\frac{t}{1-t} \frac{v_{11}}{2 v_{12}}\right)^{z_{2}} \Gamma\left(-z_{1}\right) \Gamma\left(-z_{2}\right) \Gamma\left(1-l-\varepsilon+z_{1}+z_{2}\right) \\
& \quad=\Gamma(1-l-\varepsilon)\left(1+\frac{1-t}{t} \frac{v_{12}}{2}+\frac{t}{1-t} \frac{v_{11}}{2 v_{12}}\right)^{l-1+\varepsilon} \\
& \quad=\Gamma(1-l-\varepsilon)\left(\frac{v_{12}}{2 t(1-t)}\right)^{l-1+\varepsilon}\left[1-t\left(2-\frac{2}{v_{12}}\right)+t^{2}\left(1-\frac{2}{v_{12}}+\frac{v_{11}}{v_{12}^{2}}\right)\right]^{l-1+\varepsilon} \\
& \quad=\Gamma(1-l-\varepsilon)\left(\frac{v_{12}}{2 t(1-t)}\right)^{l-1+\varepsilon}\left(1-\tau_{+} t\right)^{l-1+\varepsilon}\left(1-\tau_{-} t\right)^{l-1+\varepsilon}
\end{aligned}
$$


where we introduced the notation $\tau_{ \pm}=1-\left(1 \pm \sqrt{1-v_{11}}\right) / v_{12}$. Plugging this into integral (3.53) gives

$$
\begin{aligned}
I_{j, l}^{(1)}\left(v_{12}, v_{11} ; \varepsilon\right)= & \frac{I^{(0)}(\varepsilon)}{2^{l} v_{12}^{j}} \frac{(2-j-l-2 \varepsilon)_{j+l}}{(1-l-\varepsilon)_{l} \Gamma(j)} \\
& \times \int_{0}^{1} \mathrm{~d} t t^{j-1}(1-t)^{1-j-l-2 \varepsilon}\left(1-\tau_{+} t\right)^{l-1+\varepsilon}\left(1-\tau_{-} t\right)^{l-1+\varepsilon} .
\end{aligned}
$$

The $t$-integral is the Euler integral representation of the Appell function $F_{1}(a, b, c, d, x, y)$ :

$$
F_{1}(a, b, c, d, x, y)=\frac{\Gamma(c)}{\Gamma(a) \Gamma(d-a)} \int_{0}^{1} \mathrm{~d} t t^{a-1}(1-t)^{d-a-1}(1-x t)^{-b}(1-y t)^{-c} .
$$

Here for completeness we also present the definition of the Appell function in terms of the Pochhammer symbol

$$
F_{1}(a, b, c, d, x, y)=\sum_{m, n=0}^{\infty} \frac{(a)_{m+n}(b)_{m}(c)_{n}}{(d)_{m+n}} \frac{x^{m} y^{n}}{m ! n !} .
$$

Hence, we obtain

$$
\begin{aligned}
I_{j, l}^{(1)}\left(v_{12}, v_{11} ; \varepsilon\right)= & \frac{I^{(0)}(\varepsilon)}{2^{l} v_{12}^{j}} \frac{(2-j-l-2 \varepsilon)_{j+l}}{(1-l-\varepsilon)_{l}} \frac{\Gamma(2-j-l-2 \varepsilon)}{\Gamma(2-l-2 \varepsilon)} \\
& \times F_{1}\left(j, 1-l-\varepsilon, 1-l-\varepsilon, 2-l-2 \varepsilon, \tau_{+}, \tau_{-}\right) . \\
= & \frac{I^{(0)}(\varepsilon)}{2^{l} v_{12}^{j}} \frac{(2-l-2 \varepsilon)_{l}}{(1-l-\varepsilon)_{l}} F_{1}\left(j, 1-l-\varepsilon, 1-l-\varepsilon, 2-l-2 \varepsilon, \tau_{+}, \tau_{-}\right) \\
= & \frac{I_{l}^{(0)}(\varepsilon)}{v_{12}^{j}} F_{1}\left(j, 1-l-\varepsilon, 1-l-\varepsilon, 2-l-2 \varepsilon, \tau_{+}, \tau_{-}\right) .
\end{aligned}
$$

As in section 3.2.4 this result is valid for all $j$ and $l$ even though the derivation assumed that neither of them is a non-positive integer. Employing the transformation

$$
F_{1}\left(a, b_{1}, b_{2}, c, x, y\right)=(1-x)^{-b_{1}}(1-y)^{-b_{2}} F_{1}\left(c-a, b_{1}, b_{2}, c, \frac{x}{x-1}, \frac{y}{y-1}\right),
$$

gives the alternative representation

$$
\begin{aligned}
I_{j, l}^{(1)}\left(v_{12}, v_{11} ; \varepsilon\right)= & \frac{I_{l}^{(0)}(\varepsilon)}{v_{12}^{j}}\left(\frac{v_{11}}{v_{12}^{2}}\right)^{l-1+\varepsilon} \\
& \times F_{1}\left(2-j-l-2 \varepsilon, 1-l-\varepsilon, 1-l-\varepsilon, 2-l-2 \varepsilon, \frac{\tau_{+}}{\tau_{+}-1}, \frac{\tau_{-}}{\tau_{-}-1}\right),
\end{aligned}
$$

which is a suitable starting point for the $\varepsilon$-expansion in the case of positive integer $j$ and $l$.

For $j \in \mathbb{Z}_{\leq 0}$ the hypergeometric series terminates at $m+n=-j$, since $(j)_{m+n}=0$ otherwise. Therefore,

$$
\begin{aligned}
& F_{1}(j, 1-l-\varepsilon, 1-l-\varepsilon, 2-l-2 \varepsilon, x, y)=\sum_{m, n=0}^{\infty} \frac{(j)_{m+n}(1-l-\varepsilon)_{m}(1-l-\varepsilon)_{n}}{(2-l-2 \varepsilon)_{m+n}} \frac{x^{m}}{m !} \frac{y^{n}}{n !} \\
& \stackrel{j \in \mathbb{Z}_{\leq}}{=} \sum_{m, n \leq-j} \frac{(j)_{m+n}(1-l-\varepsilon)_{m}(1-l-\varepsilon)_{n}}{(2-l-2 \varepsilon)_{m+n}} \frac{x^{m}}{m !} \frac{y^{n}}{n !},
\end{aligned}
$$

which makes the $\varepsilon$-expansion of $I_{j, l}^{(1)}\left(v_{12}, v_{11} ; \varepsilon\right)$ trivial. 
For $l \in \mathbb{Z}_{\leq 0}$ the Appell function reduces to a sum of hypergeometric functions. It holds

$$
\begin{aligned}
& F_{1}(j, 1-l-\varepsilon, 1-l-\varepsilon, 2-l-2 \varepsilon, x, y) \\
& \stackrel{l \in \mathbb{Z}_{\leq 0}}{=} \frac{(2-2 l-2 \varepsilon)_{l}}{(1-y)^{j}} \sum_{n=0}^{-l}\left(\begin{array}{c}
-l \\
n
\end{array}\right)(-1)^{l-n} \frac{(j)_{n 2} F_{1}\left(j+n, 1-l-\varepsilon, 2-2 l-2 \varepsilon, \frac{x-y}{1-y}\right)}{(1-y)^{n}(j+l-1+2 \varepsilon)_{l+n}} .
\end{aligned}
$$

This form allows for a much simpler $\varepsilon$-expansion since we reduced the two-variable Appell function to a finite sum of genuinely one-variable functions.

\subsubsection{Double massive integral with two denominators}

An approach similar to the massless and single-massive case yields a complicated three-fold Mellin-Barnes integral (see ref. [48]). Even though this might be a good starting point for numerically establishing $\varepsilon$-expansions, there has been no successful attempt to obtain a closed analytic expression similar to the single-massive case [48]. We will close this gap in section 3.3 .1

For the purpose of $\varepsilon$-expansion, we propose the following promising new method. By restricting ourselves to $j, l \in \mathbb{N}$ we obtain a closed expression for the double massive integral for arbitrary $D$ by employing the generalized two-mass splitting formula (D.17). This reduces the double massive integral to a sum of $j+l$ single-massive integrals. For $j, l \in \mathbb{Z}_{\leq 0}$ we use eq. (D.35) for reduction to single massive integrals.

Using the generalized two-point splitting lemma [see details in appendix D] we get

$$
\begin{aligned}
& I_{j, l}^{(2)}\left(v_{12}, v_{11}, v_{22} ; \varepsilon\right)=\int \mathrm{d} \Omega_{k_{1} k_{2}} \frac{1}{\left(v_{1} \cdot k\right)^{j}\left(v_{2} \cdot k\right)^{l}}=\int \mathrm{d} \Omega_{k_{1} k_{2}} \Delta_{\mathbf{k}}^{j}\left(\mathbf{v}_{\mathbf{1}}\right) \Delta_{\mathbf{k}}^{l}\left(\mathbf{v}_{\mathbf{2}}\right) \\
& =\int \mathrm{d} \Omega_{k_{1} k_{2}}\left[\sum_{n=0}^{j-1}\left(\begin{array}{c}
l-1+n \\
l-1
\end{array}\right) \lambda_{ \pm}^{l}\left(1-\lambda_{ \pm}\right)^{n} \Delta_{\mathbf{k}}^{j-n}\left(\mathbf{v}_{\mathbf{1}}\right) \Delta_{\mathbf{k}}^{l+n}\left(\mathbf{v}_{\mathbf{3}}^{ \pm}\right)\right. \\
& \left.+\sum_{n=0}^{l-1}\left(\begin{array}{c}
j-1+n \\
j-1
\end{array}\right) \lambda_{ \pm}^{n}\left(1-\lambda_{ \pm}\right)^{j} \Delta_{\mathbf{k}}^{l-n}\left(\mathbf{v}_{\mathbf{2}}\right) \Delta_{\mathbf{k}}^{j+n}\left(\mathbf{v}_{\mathbf{3}}^{ \pm}\right)\right] \\
& =\sum_{n=0}^{j-1}\left(\begin{array}{c}
l-1+n \\
l-1
\end{array}\right) \lambda_{ \pm}^{l}\left(1-\lambda_{ \pm}\right)^{n} \int \mathrm{d} \Omega_{k_{1} k_{2}} \Delta_{\mathbf{k}}^{j-n}\left(\mathbf{v}_{\mathbf{1}}\right) \Delta_{\mathbf{k}}^{l+n}\left(\mathbf{v}_{\mathbf{3}}^{ \pm}\right) \\
& +\sum_{n=0}^{l-1}\left(\begin{array}{c}
j-1+n \\
j-1
\end{array}\right) \lambda_{ \pm}^{n}\left(1-\lambda_{ \pm}\right)^{j} \int \mathrm{d} \Omega_{k_{1} k_{2}} \Delta_{\mathbf{k}}^{l-n}\left(\mathbf{v}_{\mathbf{2}}\right) \Delta_{\mathbf{k}}^{j+n}\left(\mathbf{v}_{\mathbf{3}}^{ \pm}\right)
\end{aligned}
$$

where $\left(v_{3}^{ \pm}\right)^{2}=0$, therefore the double massive integral with $j, l \in \mathbb{N}$ can be evaluated for general $\varepsilon$ as

$$
\begin{aligned}
I_{j, l}^{(2)}\left(v_{12}, v_{11}, v_{22} ; \varepsilon\right)= & \sum_{n=0}^{j-1}\left(\begin{array}{c}
l-1+n \\
l-1
\end{array}\right) \lambda_{ \pm}^{l}\left(1-\lambda_{ \pm}\right)^{n} I_{j-n, l+n}^{(1)}\left(v_{13}^{ \pm}, v_{11} ; \varepsilon\right) \\
& +\sum_{n=0}^{l-1}\left(\begin{array}{c}
j-1+n \\
j-1
\end{array}\right) \lambda_{ \pm}^{n}\left(1-\lambda_{ \pm}\right)^{j} I_{l-n, j+n}^{(1)}\left(v_{23}^{ \pm}, v_{22} ; \varepsilon\right)
\end{aligned}
$$


where

$$
\begin{aligned}
& \lambda_{ \pm}=\frac{v_{12}-v_{11} \pm \sqrt{v_{12}^{2}-v_{11} v_{22}}}{2 v_{12}-v_{11}-v_{22}}, \\
& v_{13}^{ \pm}=\left(1-\lambda_{ \pm}\right) v_{11}+\lambda_{ \pm} v_{12}=\frac{v_{11}\left(v_{22} \pm \sqrt{v_{12}^{2}-v_{11} v_{22}}\right)-v_{12}\left(v_{12} \pm \sqrt{v_{12}^{2}-v_{11} v_{22}}\right)}{v_{11}+v_{22}-2 v_{12}}, \\
& v_{23}^{ \pm}=\left(1-\lambda_{ \pm}\right) v_{12}+\lambda_{ \pm} v_{22}=\frac{v_{22}\left(v_{11} \mp \sqrt{v_{12}^{2}-v_{11} v_{22}}\right)-v_{12}\left(v_{12} \mp \sqrt{v_{12}^{2}-v_{11} v_{22}}\right)}{v_{11}+v_{22}-2 v_{12}} .
\end{aligned}
$$

Formula (3.64) holds for both sign choices.

When the power of one of the massive propagators is negative one can derive a simpler expression for the corresponding angular integral. In particular, using eqs. (D.36) and (D.37), it holds

$$
\begin{aligned}
I_{j, l}^{(2)}\left(v_{12}, v_{11}, v_{22} ; \varepsilon\right)= & \sum_{n=0}^{-j}\left(\begin{array}{c}
-j \\
n
\end{array}\right)\left(1-\sqrt{1-v_{11}}\right)^{-j-n}\left(1-v_{11}\right)^{n / 2} \\
& \times I_{l,-n}^{(1)}\left(1-\frac{1-v_{12}}{\sqrt{1-v_{11}}}, v_{22} ; \varepsilon\right)
\end{aligned}
$$

for $j \in \mathbb{Z}_{\leq 0}$ and equivalently

$$
\begin{aligned}
I_{j, l}^{(2)}\left(v_{12}, v_{11}, v_{22} ; \varepsilon\right)= & \sum_{n=0}^{-l}\left(\begin{array}{c}
-l \\
n
\end{array}\right)\left(1-\sqrt{1-v_{22}}\right)^{-l-n}\left(1-v_{22}\right)^{n / 2} \\
& \times I_{j,-n}^{(1)}\left(1-\frac{1-v_{12}}{\sqrt{1-v_{22}}}, v_{11} ; \varepsilon\right),
\end{aligned}
$$

for $l \in \mathbb{Z}_{\leq 0}$.

\subsection{New properties of angular integrals}

In this section we review new properties of angular integrals proposed and developed in present paper for the first time. In particular, we derive: (1) hypergeometric representation of the general two denominator angular integral in $D$ dimensions; (2) differential, partial integration, and recursion relations for angular integrals. The main advantage of our findings is that it allow to reduce all known angular integrals to a small set of basis integrals.

\subsubsection{Hypergeometric representation of angular integrals}

It is known (see, e.g., detailed discussion in ref. [48]) that many angular integrals are represented in terms of hypergeometric (Gauss, Appell) functions. In this section we demonstrate how to derive the hypergeometric representation of the general angular integral having two denominators in terms of the Lauricella function $F_{B}^{(3)}$. The latter being a three-variable generalization of the Gauss and Appell function. 
For this purpose we consider generic angular integral in $D=4-2 \varepsilon$ dimensions

$$
I_{j, l}\left(v_{12}, v_{11}, v_{22} ; \varepsilon\right)=\int \mathrm{d} \Omega_{k_{1} k_{2}} \frac{1}{\left(v_{1} \cdot k\right)^{j}\left(v_{2} \cdot k\right)^{l}},
$$

where as before $\mathrm{d} \Omega_{k_{1} k_{2}}=\mathrm{d} \theta_{1} \sin ^{1-2 \varepsilon} \theta_{1} \mathrm{~d} \theta_{2} \sin ^{-2 \varepsilon} \theta_{2}$ and $v_{i j}=v_{i} \cdot v_{j}$.

Using Feynman parametrization one gets

$$
I_{j, l}\left(v_{12}, v_{11}, v_{22} ; \varepsilon\right)=B(j, l) \int_{0}^{1} \mathrm{~d} x_{1} x_{1}^{j-1} \int_{0}^{1} \mathrm{~d} x_{2} x_{2}^{l-1} \delta\left(1-x_{1}-x_{2}\right) I_{j+l}^{(1)}\left(w_{12} ; \varepsilon\right),
$$

where $w_{12}=\left(x_{1} v_{1}+x_{2} v_{2}\right)^{2}$ is the Feynman "mass parameter".

The one denominator massive integral $I_{j+l}^{(1)}(v, \varepsilon)$ is given by

$$
I_{j+l}^{(1)}(v ; \varepsilon)=I^{(0)}(\varepsilon){ }_{2} F_{1}\left(\frac{j+l}{2}, \frac{j+l+1}{2}, \frac{3}{2}-\varepsilon, 1-v\right) .
$$

Due to the $\delta$-function it follows $2 x_{1} x_{2}=1-x_{1}^{2}-x_{2}^{2}$, and therefore $w_{12}$ can be presented in the form, where the variables $x_{1}$ and $x_{2}$ are separated,

$$
w_{12}=x_{1}^{2} v_{11}+2 x_{1} x_{2} v_{12}+x_{2}^{2} v_{22}=v_{12}-x_{1}^{2}\left(v_{12}-v_{11}\right)-x_{2}^{2}\left(v_{12}-v_{22}\right) .
$$

Representation (3.71) is very useful since it leads to a Mellin-Barnes integral, which is considerably simpler than that derived in ref. [48]. In addition, it can be expressed in terms of the known hypergeometric function $F_{B}^{(3)}$. Using eq. (3.71), the Mellin-Barnes representation of the Gauss hypergeometric function

$$
{ }_{2} F_{1}(a, b, c, x)=\int_{-i \infty}^{i \infty} \frac{\mathrm{d} z}{2 \pi i} \frac{(a)_{z}(b)_{z}}{(c)_{z}} \Gamma(-z)(-x)^{z},
$$

and the Newton-Binomi-Mellin integral

$$
(a+b+c)^{z}=\frac{1}{\Gamma(-z)} \int_{-i \infty}^{i \infty} \frac{\mathrm{d} z_{1}}{2 \pi i} \int_{-i \infty}^{i \infty} \frac{\mathrm{d} z_{2}}{2 \pi i} a^{z-z_{1}-z_{2}} b^{z_{1}} c^{z_{2}} \Gamma\left(-z_{1}\right) \Gamma\left(-z_{2}\right) \Gamma\left(-z+z_{1}+z_{2}\right)
$$

we obtain

$$
\begin{aligned}
I_{j+l}^{(1)}\left(w_{12} ; \varepsilon\right)= & I^{(0)}(\varepsilon) \int_{-i \infty}^{i \infty} \frac{\mathrm{d} z}{2 \pi i} \Gamma(-z) \\
& \times\left(-\left(1-v_{12}\right)-x_{1}^{2}\left(v_{12}-v_{11}\right)-x_{2}^{2}\left(v_{12}-v_{22}\right)\right)^{z} \frac{\left(\frac{j+l}{2}\right)_{z}\left(\frac{j+l+1}{2}\right)_{z}}{\left(\frac{3}{2}-\varepsilon\right)_{z}} \\
= & I^{(0)}(\varepsilon) \int_{-i \infty}^{i \infty} \frac{\mathrm{d} z}{2 \pi i} \int_{-i \infty}^{i \infty} \frac{\mathrm{d} z_{1}}{2 \pi i} \int_{-i \infty}^{i \infty} \frac{\mathrm{d} z_{2}}{2 \pi i} \Gamma\left(-z_{1}\right) \Gamma\left(-z_{2}\right) \Gamma\left(-z+z_{1}+z_{2}\right) \\
& \times\left(v_{11}-v_{12}\right)^{z_{1}}\left(v_{22}-v_{12}\right)^{z_{2}}\left(v_{12}-1\right)^{z-z_{1}-z_{2}} \frac{\left(\frac{j+l}{2}\right)_{z}\left(\frac{j+l+1}{2}\right)_{z}}{\left(\frac{3}{2}-\varepsilon\right)_{z}} x_{1}^{2 z_{1}} x_{2}^{2 z_{2}} \\
= & I^{(0)}(\varepsilon) \int_{-i \infty}^{i \infty} \frac{\mathrm{d} z_{1}}{2 \pi i} \int_{-i \infty}^{i \infty} \frac{\mathrm{d} z_{2}}{2 \pi i} \int_{-i \infty}^{i \infty} \frac{\mathrm{d} z_{3}}{2 \pi i} \Gamma\left(-z_{1}\right) \Gamma\left(-z_{2}\right) \Gamma\left(-z_{3}\right) \\
& \times\left(v_{11}-v_{12}\right)^{z_{1}}\left(v_{22}-v_{12}\right)^{z_{2}}\left(v_{12}-1\right)^{z_{3}} \frac{\left(\frac{j+l}{2}\right)_{z_{1}+z_{2}+z_{3}}\left(\frac{j+l+1}{2}\right)_{z_{1}+z_{2}+z_{3}}}{\left(\frac{3}{2}-\varepsilon\right)_{1}^{2 z_{1}} x_{2}^{2 z_{2}} .} .
\end{aligned}
$$


In the last step of derivation of eq. (3.74) we changed variable $z \rightarrow z_{3}=z-z_{1}-z_{2}$. The combination of the Pochhammer symbols can be presented in the following form

$$
\begin{aligned}
\frac{\left(\frac{j+l}{2}\right)_{z_{1}+z_{2}+z_{3}}\left(\frac{j+l+1}{2}\right)_{z_{1}+z_{2}+z_{3}}}{\left(\frac{3}{2}-\varepsilon\right)_{z_{1}+z_{2}+z_{3}}}= & \frac{4^{-z_{1}-z_{2}}(j+l)_{2 z_{1}+2 z_{2}}}{\left(\frac{3}{2}-\varepsilon\right)_{z_{1}+z_{2}}} \\
& \times \frac{\left(\frac{j+l}{2}+z_{1}+z_{2}\right)_{z_{3}}\left(\frac{j+l+1}{2}+z_{1}+z_{2}\right)_{z_{3}}}{\left(\frac{3}{2}-\varepsilon+z_{1}+z_{2}\right)_{z_{3}}},
\end{aligned}
$$

whereby the $z_{3}$ integral becomes a Gauss hypergeometric function,

$$
\begin{aligned}
I_{j+l}^{(1)}\left(w_{12} ; \varepsilon\right)= & I^{(0)}(\varepsilon) \int_{-i \infty}^{i \infty} \frac{\mathrm{d} z_{1}}{2 \pi i} \int_{-i \infty}^{i \infty} \frac{\mathrm{d} z_{2}}{2 \pi i}\left(\frac{v_{11}-v_{12}}{4}\right)^{z_{1}}\left(\frac{v_{22}-v_{12}}{4}\right)^{z_{2}} \\
& \times \Gamma\left(-z_{1}\right) \Gamma\left(-z_{2}\right) \frac{(j+l)_{2 z_{1}+2 z_{2}}}{\left(\frac{3}{2}-\varepsilon\right)_{z_{1}+z_{2}}^{2 z_{1}} x_{2}^{2 z_{2}}} \\
& \times{ }_{2} F_{1}\left(\frac{j+l}{2}+z_{1}+z_{2}, \frac{j+l+1}{2}+z_{1}+z_{2}, \frac{3}{2}-\varepsilon+z_{1}+z_{2}, 1-v_{12}\right) .
\end{aligned}
$$

Next to reduce the number of occurrences of the integration variables $z_{1}$ and $z_{2}$ in the arguments of the Gauss hypergeometric function we perform the Euler transformation

$$
\begin{aligned}
& { }_{2} F_{1}\left(\frac{j+l}{2}+z_{1}+z_{2}, \frac{j+l+1}{2}+z_{1}+z_{2}, \frac{3}{2}-\varepsilon+z_{1}+z_{2}, 1-v_{12}\right) \\
& =v_{12}^{1-j-l-\varepsilon-z_{1}-z_{2}}{ }_{2} F_{1}\left(\frac{3-j-l-2 \varepsilon}{2}, \frac{2-j-l-2 \varepsilon}{2}, \frac{3}{2}-\varepsilon+z_{1}+z_{2}, 1-v_{12}\right) .
\end{aligned}
$$

Introducing again the Mellin-Barnes representation of the Gauss hypergeometric function (3.72) and combining the Pochhammer symbols leads to

$$
\begin{aligned}
I_{j+l}^{(1)}\left(\left(x_{1} v_{1}+x_{2} v_{2}\right)^{2} ; \varepsilon\right)= & I^{(0)}(\varepsilon) v_{12}^{1-j-l-\varepsilon} \int_{-i \infty}^{i \infty} \frac{\mathrm{d} z_{1}}{2 \pi i} \int_{-i \infty}^{i \infty} \frac{\mathrm{d} z_{2}}{2 \pi i} \int_{-i \infty}^{i \infty} \frac{\mathrm{d} z_{3}}{2 \pi i} \\
& \times \Gamma\left(-z_{1}\right) \Gamma\left(-z_{2}\right) \Gamma\left(-z_{3}\right)\left(\frac{v_{11}-v_{12}}{4 v_{12}}\right)^{z_{1}}\left(\frac{v_{22}-v_{12}}{4 v_{12}}\right)^{z_{2}}\left(\frac{v_{12}-1}{4}\right)^{z_{3}} \\
& \times x_{1}^{2 z_{1}} x_{2}^{2 z_{2}} \frac{(j+l)_{2 z_{1}+2 z_{2}}(2-j-l-2 \varepsilon)_{2 z_{3}}}{\left(\frac{3}{2}-\varepsilon\right)_{z_{1}+z_{2}+z_{3}}}
\end{aligned}
$$

Plugging this into expression (3.69) for $I_{j, l}$ and evaluating the Feynman parameter integral as

$$
\frac{1}{B(j, l)} \int_{0}^{1} \mathrm{~d} x_{1} x_{1}^{j-1} \int_{0}^{1} \mathrm{~d} x_{2} x_{2}^{l-1} \delta\left(1-x_{1}-x_{2}\right) x_{1}^{2 z_{1}} x_{2}^{2 z_{2}}=\frac{(j)_{2 z_{1}}(l)_{2 z_{2}}}{(j+l)_{2 z_{1}+2 z_{2}}},
$$

yields the three-fold Mellin-Barnes representation

$$
\begin{aligned}
I_{j, l}\left(v_{12}, v_{11}, v_{22} ; \varepsilon\right)= & I^{(0)}(\varepsilon) v_{12}^{1-j-l-\varepsilon} \int_{-i \infty}^{i \infty} \frac{\mathrm{d} z_{1}}{2 \pi i} \int_{-i \infty}^{i \infty} \frac{\mathrm{d} z_{2}}{2 \pi i} \int_{-i \infty}^{i \infty} \frac{\mathrm{d} z_{3}}{2 \pi i} \Gamma\left(-z_{1}\right) \Gamma\left(-z_{2}\right) \Gamma\left(-z_{3}\right) \\
& \times\left(\frac{v_{11}-v_{12}}{4 v_{12}}\right)^{z_{1}}\left(\frac{v_{22}-v_{12}}{4 v_{12}}\right)^{z_{2}}\left(\frac{v_{12}-1}{4}\right)^{z_{3}} \frac{(j)_{2 z_{1}}(l)_{2 z_{2}}(2-j-l-2 \varepsilon)_{2 z_{3}}}{\left(\frac{3}{2}-\varepsilon\right)_{z_{1}+z_{2}+z_{3}}} .
\end{aligned}
$$


Upon employing the duplication identity $(2 x)_{2 n}=2^{2 n}(x)_{n}\left(x+\frac{1}{2}\right)_{n}$ on each Pochhammer symbol in the numerator we obtain the hypergeometric representation of $I_{j, l}$ in term of the Lauricella function $F_{B}^{(3)}$ :

$$
I_{j, l}\left(v_{12}, v_{11}, v_{22} ; \varepsilon\right)=\frac{2 \pi}{1-2 \varepsilon} v_{12}^{1-j-l-\varepsilon} F_{B}^{(3)}\left(a_{1}, a_{2}, a_{3}, b_{1}, b_{2}, b_{3}, c ; x_{1}, x_{2}, x_{3}\right),
$$

where

$$
\begin{aligned}
& a_{1}=\frac{j}{2}, \quad a_{2}=\frac{l}{2}, \quad a_{3}=\frac{3}{2}-a_{1}-a_{2}-\varepsilon, \quad b_{1}=a_{1}+\frac{1}{2}, \quad b_{2}=a_{2}+\frac{1}{2}, \quad b_{3}=a_{3}-\frac{1}{2}, \\
& c=a_{1}+a_{2}+a_{3}, \quad x_{1}=1-\frac{v_{11}}{v_{12}}, \quad x_{2}=1-\frac{v_{22}}{v_{12}}, \quad x_{3}=1-v_{12} .
\end{aligned}
$$

Here the Lauricella function $F_{B}^{(3)}$ is equivalently defined as the Mellin-Barnes integral

$$
\begin{aligned}
F_{B}^{(3)}\left(a_{1}, a_{2}, a_{3}, b_{1}, b_{2}, b_{3}, c, x_{1}, x_{2}, x_{3}\right)= & \int_{-i \infty}^{i \infty} \frac{\mathrm{d} z_{1}}{2 \pi i} \int_{-i \infty}^{i \infty} \frac{\mathrm{d} z_{2}}{2 \pi i} \int_{-i \infty}^{i \infty} \frac{\mathrm{d} z_{3}}{2 \pi i} \Gamma\left(-z_{1}\right) \Gamma\left(-z_{2}\right) \Gamma\left(-z_{3}\right) \\
& \times\left(-x_{1}\right)^{z_{1}}\left(-x_{2}\right)^{z_{2}}\left(-x_{3}\right)^{z_{3}} \\
& \times \frac{\left(a_{1}\right)_{z_{1}}\left(a_{2}\right)_{z_{2}}\left(a_{3}\right)_{z_{3}}\left(b_{1}\right)_{z_{1}}\left(b_{2}\right)_{z_{2}}\left(b_{3}\right)_{z_{3}}}{(c)_{z_{1}+z_{2}+z_{3}}}
\end{aligned}
$$

or as the sum in terms of the Pochhammer symbols

$$
\begin{aligned}
F_{B}^{(3)}\left(a_{1}, a_{2}, a_{3}, b_{1}, b_{2}, b_{3}, c, x_{1}, x_{2}, x_{3}\right)= & \sum_{m, n, p=0}^{\infty} \frac{\left(a_{1}\right)_{m}\left(a_{2}\right)_{n}\left(a_{3}\right)_{p}\left(b_{1}\right)_{m}\left(b_{2}\right)_{n}\left(b_{3}\right)_{p}}{(c)_{m+n+p}} \\
& \times \frac{x_{1}^{m}}{m !} \frac{x_{2}^{n}}{n !} \frac{x_{3}^{p}}{p !} .
\end{aligned}
$$

Eqs. (3.81)-(3.84) represent the main result of this section - Hypergeometric representation for generic angular integral with two denominators without referring to their massshell properties (i.e. they are massless or massive). In appendix E we explicitly demonstrate the consistency with known special cases.

\subsubsection{Partial differential identities}

In this section we derive differential identities involving angular integrals, which help to establish recurrence relations between the latter. Note, there were before some efforts to obtain differential relations between Neerven-type integrals in literature (see, e.g., ref. [66]). Here we perform a study of differential properties of angular integrals in a systematic way. For that purpose we consider the generic angular integral with two denominators

$$
I_{j, l}\left(v_{12}, v_{11}, v_{22} ; \varepsilon\right)=\int \mathrm{d} \Omega_{k_{1} k_{2}} \frac{1}{\left(v_{1} \cdot k\right)^{j}\left(v_{2} \cdot k\right)^{l}}=\int \mathrm{d} \Omega_{k_{1} k_{2}} \Delta_{\mathbf{k}}^{j}\left(\mathbf{v}_{\mathbf{1}}\right) \Delta_{\mathbf{k}}^{l}\left(\mathbf{v}_{\mathbf{2}}\right) .
$$

It is convenient to choose coordinates such that

$$
\begin{aligned}
& \Delta_{\mathbf{k}}\left(\mathbf{v}_{\mathbf{1}}\right)=\frac{1}{1-\mathbf{v}_{\mathbf{1}} \cdot \mathbf{k}}=\frac{1}{1-\beta_{1} \cos \theta_{1}}, \\
& \Delta_{\mathbf{k}}\left(\mathbf{v}_{\mathbf{2}}\right)=\frac{1}{1-\mathbf{v}_{\mathbf{2}} \cdot \mathbf{k}}=\frac{1}{1-\beta_{2} \cos \vartheta \cos \theta_{1}-\beta_{2} \sin \vartheta \sin \theta_{1} \cos \theta_{2}},
\end{aligned}
$$


with

$$
\begin{aligned}
\beta_{1} & =\sqrt{1-v_{11}}, \quad \beta_{2}=\sqrt{1-v_{22}}, \\
\cos \vartheta & =\frac{1-v_{12}}{\sqrt{1-v_{11}} \sqrt{1-v_{22}}}, \\
\Delta_{12} & =\left(\beta_{1} \beta_{2} \sin \vartheta\right)^{2}=\left(1-v_{11}\right)\left(1-v_{22}\right)-\left(1-v_{12}\right)^{2} .
\end{aligned}
$$

Taking partial derivatives of the propagators gives

$$
\begin{aligned}
\frac{\partial}{\partial \beta_{1}} \Delta_{\mathbf{k}}\left(\mathbf{v}_{\mathbf{1}}\right) & =\cos \theta_{1} \Delta_{\mathbf{k}}^{2}\left(\mathbf{v}_{\mathbf{1}}\right) \\
\frac{\partial}{\partial \beta_{2}} \Delta_{\mathbf{k}}\left(\mathbf{v}_{\mathbf{2}}\right) & =\left(\cos \vartheta \cos \theta_{1}+\sin \vartheta \sin \theta_{1} \cos \theta_{2}\right) \Delta_{\mathbf{k}}^{2}\left(\mathbf{v}_{\mathbf{2}}\right), \\
\frac{\partial}{\partial \vartheta} \Delta_{\mathbf{k}}\left(\mathbf{v}_{\mathbf{2}}\right) & =\beta_{2}\left(-\sin \vartheta \cos \theta_{1}+\cos \vartheta \sin \theta_{1} \cos \theta_{2}\right) \Delta_{\mathbf{k}}^{2}\left(\mathbf{v}_{\mathbf{2}}\right)
\end{aligned}
$$

Expressing appearing angular prefactors through the propagators yields

$$
\begin{aligned}
\frac{\partial}{\partial \beta_{i}} \Delta_{\mathbf{k}}\left(\mathbf{v}_{\mathbf{i}}\right) & =\frac{1}{\beta_{i}} L_{\mathbf{k}}\left(\mathbf{v}_{\mathbf{i}}\right) \Delta_{\mathbf{k}}^{2}\left(\mathbf{v}_{\mathbf{i}}\right) \\
\frac{\partial}{\partial \vartheta} \Delta_{\mathbf{k}}\left(\mathbf{v}_{\mathbf{2}}\right) & =\cot \vartheta\left[L_{\mathbf{k}}\left(\mathbf{v}_{\mathbf{2}}\right)-\frac{\beta_{2}}{\beta_{1} \cos \vartheta} L_{\mathbf{k}}\left(\mathbf{v}_{\mathbf{1}}\right)\right] \Delta_{\mathbf{k}}^{2}\left(\mathbf{v}_{\mathbf{2}}\right)
\end{aligned}
$$

where $L_{\mathbf{k}}(\mathbf{v})=1-\Delta_{\mathbf{k}}^{-1}(\mathbf{v})$. Using these differential identities we can calculate the derivatives of $I_{j, l}$ with respect to $\beta_{1}, \beta_{2}$, and $\vartheta$. We have

$$
\begin{aligned}
\frac{\partial}{\partial \beta_{1}} I_{j, l} & =\int \mathrm{d} \Omega_{k_{1} k_{2}} \frac{\partial}{\partial \beta_{1}} \Delta_{\mathbf{k}}^{j}\left(\mathbf{v}_{\mathbf{1}}\right) \Delta_{\mathbf{k}}^{l}\left(\mathbf{v}_{\mathbf{2}}\right) \\
& =\frac{j}{\beta_{1}} \int \mathrm{d} \Omega_{k_{1} k_{2}} L_{\mathbf{k}}\left(\mathbf{v}_{\mathbf{1}}\right) \Delta_{\mathbf{k}}^{j-1}\left(\mathbf{v}_{\mathbf{1}}\right) \Delta_{\mathbf{k}}^{l}\left(\mathbf{v}_{\mathbf{2}}\right) \Delta_{\mathbf{k}}^{2}\left(\mathbf{v}_{\mathbf{1}}\right) \\
& =\frac{j}{\beta_{1}}\left(I_{j+1, l}-I_{j, l}\right),
\end{aligned}
$$

and by analogy

$$
\begin{aligned}
\frac{\partial}{\partial \beta_{2}} I_{j, l} & =\frac{l}{\beta_{2}}\left(I_{j, l+1}-I_{j, l}\right), \\
\frac{\partial}{\partial \vartheta} I_{j, l} & =l \cot \vartheta\left(\left(1-\frac{\beta_{2}}{\beta_{1} \cos \vartheta}\right) I_{j, l+1}-I_{j, l}+\frac{\beta_{2}}{\beta_{1} \cos \vartheta} I_{j-1, l+1}\right) .
\end{aligned}
$$

Due to rotational invariance the angular integral $I_{j, l}$ does not change under replacement of orientation of vectors $v_{1}$ and $v_{2}$ with respect to integration momentum $k$. This helps to get the identity equivalent to eq. (3.96):

$$
\frac{\partial}{\partial \vartheta} I_{j, l}=j \cot \vartheta\left(\left(1-\frac{\beta_{1}}{\beta_{2} \cos \vartheta}\right) I_{j+1, l}-I_{j, l}+\frac{\beta_{1}}{\beta_{2} \cos \vartheta} I_{j+1, l-1}\right) .
$$


Next, we derive the differential identities for angular integrals with respect to the set of variables $\left(v_{11}, v_{22}, v_{12}\right)$ using relations (3.88) and

$$
\begin{aligned}
\frac{\partial}{\partial v_{12}} & =\frac{1}{\beta_{1} \beta_{2} \sin \vartheta} \frac{\partial}{\partial \vartheta}, \\
\frac{\partial}{\partial v_{11}} & =-\frac{1}{2 \beta_{1}} \frac{\partial}{\partial \beta_{1}}-\frac{\cot \vartheta}{2 \beta_{1}^{2}} \frac{\partial}{\partial \vartheta}, \\
\frac{\partial}{\partial v_{22}} & =-\frac{1}{2 \beta_{2}} \frac{\partial}{\partial \beta_{2}}-\frac{\cot \vartheta}{2 \beta_{2}^{2}} \frac{\partial}{\partial \vartheta} .
\end{aligned}
$$

One gets:

$$
\begin{aligned}
\frac{\partial}{\partial v_{12}} I_{j, l} & =\frac{l}{\Delta_{12}}\left[\left(v_{22}-v_{12}\right) I_{j, l+1}-\left(1-v_{12}\right) I_{j, l}+\left(1-v_{22}\right) I_{j-1, l+1}\right] \\
& =\frac{j}{\Delta_{12}}\left[\left(v_{11}-v_{12}\right) I_{j+1, l}-\left(1-v_{12}\right) I_{j, l}+\left(1-v_{11}\right) I_{j+1, l-1}\right], \\
\frac{\partial}{\partial v_{11}} I_{j, l} & =\frac{j}{2 \Delta_{12}}\left[\left(v_{22}-v_{12}\right) I_{j+1, l}+\left(1-v_{22}\right) I_{j, l}-\left(1-v_{12}\right) I_{j+1, l-1}\right], \\
\frac{\partial}{\partial v_{22}} I_{j, l} & =\frac{l}{2 \Delta_{12}}\left[\left(v_{11}-v_{12}\right) I_{j, l+1}+\left(1-v_{11}\right) I_{j, l}-\left(1-v_{12}\right) I_{j-1, l+1}\right] .
\end{aligned}
$$

Two independent ways of calculating $\frac{\partial}{\partial v_{12}} I_{j, l}$ [see eqs. (3.101) and (3.102)] lead to the consistency relation

$$
\begin{aligned}
& l\left(v_{22}-v_{12}\right) I_{j, l+1}-l\left(1-v_{12}\right) I_{j, l}+l\left(1-v_{22}\right) I_{j-1, l+1} \\
& \quad=j\left(v_{11}-v_{12}\right) I_{j+1, l}-j\left(1-v_{12}\right) I_{j, l}+j\left(1-v_{11}\right) I_{j+1, l-1} .
\end{aligned}
$$

This is a first algebraic (recurrence) relation between $I_{j, l}$ with different indices.

From eqs. (3.101)-(3.104) we deduce the following identities

$$
\begin{aligned}
2 l \frac{\partial}{\partial v_{11}} I_{j, l+1} & =j \frac{\partial}{\partial v_{12}} I_{j+1, l}, \\
2 j \frac{\partial}{\partial v_{22}} I_{j+1, l} & =l \frac{\partial}{\partial v_{12}} I_{j, l+1} .
\end{aligned}
$$

Combining equations (3.106) and (3.107), we find that $I_{j, l}\left(v_{12}, v_{11}, v_{22} ; \varepsilon\right)$ obeys the second order partial differential equation (PDE)

$$
\left(\frac{\partial^{2}}{\partial v_{12}^{2}}-4 \frac{\partial^{2}}{\partial v_{11} \partial v_{22}}\right) I_{j, l}\left(v_{12}, v_{11}, v_{22} ; \varepsilon\right)=0 .
$$

Introducing the light-cone coordinates $v_{ \pm}=\frac{1}{2}\left(v_{11} \pm v_{22}\right)$ this PDE can be written in the standard form

$$
\left(\frac{\partial^{2}}{\partial v_{+}^{2}}-\frac{\partial^{2}}{\partial v_{-}^{2}}-\frac{\partial^{2}}{\partial v_{12}^{2}}\right) I_{j, l}\left(v_{12}, v_{+}+v_{-}, v_{+}-v_{-} ; \varepsilon\right)=0
$$

We see that $I_{j, l}\left(v_{12}, v_{+}+v_{-}, v_{+}-v_{-} ; \varepsilon\right)$ satisfies a two-dimensional homogenous wave equation with "time" $v_{+}$and "speed of light" $c=1$. The "light-cone" is given by $0=$ 
$v_{+}^{2}-v_{-}^{2}-v_{12}^{2}=v_{11} v_{22}-v_{12}^{2}$. It is the surface of vanishing symmetric rank-2 Gram determinant $G=-X=v_{11} v_{22}-v_{12}^{2}$. Since the PDE is independent of $\varepsilon$, it must be fulfilled independently at all orders in the $\varepsilon$-expansion. Therefore, it could serve as a consistency check for individual terms in the $\varepsilon$-expansion.

Up until now we only considered the dependence on the indices $j$ and $l$. However, we will see that the dependence of $I_{j, l}$ on $\varepsilon$ is structurally quite similar. A good starting point is the representation

$$
\begin{aligned}
I_{j, l}= & \frac{1}{B(j, l)} I^{(0)}(\varepsilon) \int_{0}^{1} \mathrm{~d} x_{1} x_{1}^{j-1} \int_{0}^{1} \mathrm{~d} x_{2} x_{2}^{l-1} \delta\left(1-x_{1}-x_{2}\right) \\
& \times{ }_{2} F_{1}\left(\frac{j+l}{2}, \frac{j+l+1}{2}, \frac{3}{2}-\varepsilon, 1-x_{1}^{2} v_{11}-x_{2}^{2} v_{22}-2 x_{1} x_{2} v_{12}\right) .
\end{aligned}
$$

Calculating the derivative with respect to $v_{12}$ using the identity

$$
\frac{\mathrm{d}}{\mathrm{d} x}{ }_{2} F_{1}(a, b, c, x)=\frac{a b}{c}{ }_{2} F_{1}(a+1, b+1, c+1, x),
$$

yields

$$
\frac{\partial}{\partial v_{12}} I_{j, l}(\varepsilon)=-\frac{j l}{1-2 \varepsilon} I_{j+1, l+1}(\varepsilon-1) .
$$

Using either eq. (3.101) or eq. (3.102) to express $\partial I_{j, l} / \partial v_{12}$ results in an algebraic equation for $I_{j, l}(\varepsilon-1)$ in terms of $I_{j^{\prime}, l^{\prime}}(\varepsilon)$. Another way to obtain a formula of this kind is to start from the original angular integral and to express the additional factor $\sin ^{2} \theta_{1} \sin ^{2} \theta_{2}$ in terms of propagators:

$$
\begin{aligned}
\sin ^{2} \theta_{1} \sin ^{2} \theta_{2}= & 1-\frac{1}{\Delta_{12}}\left[\beta_{1}^{2} L_{\mathbf{k}}^{2}\left(\mathbf{v}_{\mathbf{2}}\right)+\beta_{2}^{2} L_{\mathbf{k}}^{2}\left(\mathbf{v}_{\mathbf{1}}\right)-2 \beta_{1} \beta_{2} \cos \vartheta L_{\mathbf{k}}\left(\mathbf{v}_{\mathbf{1}}\right) L_{\mathbf{k}}\left(\mathbf{v}_{\mathbf{2}}\right)\right] \\
= & \frac{1}{\Delta_{12}}\left[v_{11} v_{22}-v_{12}^{2}-\left(1-v_{11}\right) \Delta_{\mathbf{k}}^{-2}\left(\mathbf{v}_{\mathbf{2}}\right)-\left(1-v_{22}\right) \Delta_{\mathbf{k}}^{-2}\left(\mathbf{v}_{\mathbf{1}}\right)\right. \\
& +2\left(v_{12}-v_{11}\right) \Delta_{\mathbf{k}}^{-1}\left(\mathbf{v}_{\mathbf{2}}\right)+2\left(v_{12}-v_{22}\right) \Delta_{\mathbf{k}}^{-1}\left(\mathbf{v}_{\mathbf{1}}\right) \\
& \left.+2\left(1-v_{12}\right) \Delta_{\mathbf{k}}^{-1}\left(\mathbf{v}_{\mathbf{1}}\right) \Delta_{\mathbf{k}}^{-1}\left(\mathbf{v}_{\mathbf{2}}\right)\right]
\end{aligned}
$$

Next, we use eq. (3.113) to obtain the dimensional recurrence identity for angular integral $I_{j, l}(\varepsilon)$ :

$$
\begin{aligned}
I_{j, l}(\varepsilon-1)= & \int \mathrm{d} \Omega_{k_{1} k_{2}} \sin ^{2} \theta_{1} \sin ^{2} \theta_{2} \Delta_{\mathbf{k}}^{j}\left(\mathbf{v}_{\mathbf{1}}\right) \Delta_{\mathbf{k}}^{l}\left(\mathbf{v}_{\mathbf{2}}\right) \\
= & \frac{1}{\Delta_{12}}\left[\left(v_{11} v_{22}-v_{12}^{2}\right) I_{j, l}(\varepsilon)-\left(1-v_{11}\right) I_{j, l-2}(\varepsilon)-\left(1-v_{22}\right) I_{j-2, l}(\varepsilon)\right. \\
& \left.+2\left(v_{12}-v_{11}\right) I_{j, l-1}(\varepsilon)+2\left(v_{12}-v_{22}\right) I_{j-1, l}(\varepsilon)+2\left(1-v_{12}\right) I_{j-1, l-1}(\varepsilon)\right] .
\end{aligned}
$$

Here we take into account that

$$
\mathrm{d} \Omega_{k_{1} k_{2}}(\varepsilon) \sin ^{2} \theta_{1} \sin ^{2} \theta_{2} \equiv \mathrm{d} \Omega_{k_{1} k_{2}}(\varepsilon-1) .
$$


Now we are in the position to derive the second dimensional recurrence identity, which complements the identity (3.114). Doing this we start again with eq. (3.110) and shift dimension $\varepsilon \rightarrow \varepsilon+1$. Next, using the contiguos neighbours relation of the Gauss hypergeometric function

$$
{ }_{2} F_{1}(a, b, c-1, x)=\frac{1}{c-1}\left[a_{2} F_{1}(a+1, b, c, x)-(a-c+1){ }_{2} F_{1}(a, b, c, x)\right],
$$

we expand the angular integral $I_{j, l}(\varepsilon+1)$ into two terms:

$$
\begin{aligned}
I_{j, l}\left(v_{12}, v_{11}, v_{22} ; \varepsilon+1\right)= & \frac{1}{B(j, l)} I^{(0)}(\varepsilon+1) \int_{0}^{1} \mathrm{~d} x_{1} x_{1}^{j-1} \int_{0}^{1} \mathrm{~d} x_{2} x_{2}^{l-1} \delta\left(1-x_{1}-x_{2}\right) \\
& \times{ }_{2} F_{1}\left(\frac{j+l}{2}, \frac{j+l+1}{2}, \frac{1}{2}-\varepsilon, 1-w_{12}\right) \\
= & \frac{1}{B(j, l)} I^{(0)}(\varepsilon+1) \int_{0}^{1} \mathrm{~d} x_{1} x_{1}^{j-1} \int_{0}^{1} \mathrm{~d} x_{2} x_{2}^{l-1} \delta\left(1-x_{1}-x_{2}\right) \\
& \times \frac{1}{1-2 \varepsilon}\left[(j+l)_{2} F_{1}\left(\frac{j+l+1}{2}, \frac{j+l+2}{2}, \frac{3}{2}-\varepsilon, w_{12}\right)\right. \\
& \left.-(j+l-1+2 \varepsilon)_{2} F_{1}\left(\frac{j+l}{2}, \frac{j+l+1}{2}, \frac{3}{2}-\varepsilon, w_{12}\right)\right] .
\end{aligned}
$$

The second term in eq. (3.117) directly converts into integral $I_{j, l}(\varepsilon)$. In case of the first term we apply the identity for the integrand

$$
\delta\left(1-x_{1}-x_{2}\right) x_{1}^{m} x_{2}^{n}=\delta\left(1-x_{1}-x_{2}\right)\left(x_{1}^{m+1} x_{2}^{n}+x_{1}^{m} x_{2}^{n+1}\right),
$$

to obtain a sum of two angular integrals $I_{j+1, l}$ and $I_{j, l+1}$. Finally, using

$$
I^{(0)}(\varepsilon+1)=\frac{2 \pi}{1-2(\varepsilon+1)}=-\frac{1-2 \varepsilon}{1+2 \varepsilon} I^{(0)}(\varepsilon)
$$

we get

$$
I_{j, l}(\varepsilon+1)=\frac{j+l-1+2 \varepsilon}{1+2 \varepsilon} I_{j, l}(\varepsilon)-\frac{j}{1+2 \varepsilon} I_{j+1, l}(\varepsilon)-\frac{l}{1+2 \varepsilon} I_{j, l+1}(\varepsilon) .
$$

The latter identity is the second dimensional recurrence identity, which is more compact than the first one (3.114).

We finalize this section with the derivation of the differential equations for index and dimension rising operators. In particular, using the set of identities (3.101)-(3.104) we introduce the index $j / l$ rising differential operators at fixed dimension:

$$
\begin{aligned}
& \hat{D}_{1, j}=-2\left(1-v_{11}\right) \frac{\partial}{\partial v_{11}}-\left(1-v_{12}\right) \frac{\partial}{\partial v_{12}}+j, \\
& \hat{D}_{2, l}=-2\left(1-v_{22}\right) \frac{\partial}{\partial v_{22}}-\left(1-v_{12}\right) \frac{\partial}{\partial v_{12}}+l,
\end{aligned}
$$

which obey the following index rising differential equations :

$$
\begin{aligned}
\hat{D}_{1, j} I_{j, l}(\varepsilon) & =j I_{j+1, l}(\varepsilon), \\
\hat{D}_{2, l} I_{j, l}(\varepsilon) & =l I_{j, l+1}(\varepsilon) .
\end{aligned}
$$


Next, we can sum up eqs. (3.123) and (3.124), where r.h.s. is further simplified using identity (3.120):

$$
j I_{j+1, l}(\varepsilon)+l I_{j, l+1}(\varepsilon)=(j+l-1+2 \varepsilon) I_{j, l}(\varepsilon)-(1+2 \varepsilon) I_{j, l}(\varepsilon+1) .
$$

Now it is convenient to define the dimension rising differential operator at fixed indices:

$$
\hat{D}_{\varepsilon}=\sum_{v=v_{11}, v_{12}, v_{22}} 2(1-v) \frac{\partial}{\partial v}-1+2 \varepsilon,
$$

which obeys the following dimension rising differential equation:

$$
\hat{D}_{\varepsilon} I_{j, l}(\varepsilon)=(1+2 \varepsilon) I_{j, l}(\varepsilon+1) .
$$

\subsubsection{Partial integration identities}

Partial integration identities between angular integrals are another and complementary possibility to differential identities derived in previous section. We will perform the partial integration with respect to $\theta_{1}$. We will need

$$
\begin{aligned}
& \frac{\partial}{\partial \theta_{1}} \Delta_{\mathbf{k}}\left(\mathbf{v}_{\mathbf{1}}\right)=-\beta_{1} \sin \theta_{1} \Delta_{\mathbf{k}}^{2}\left(\mathbf{v}_{\mathbf{1}}\right) \\
& \frac{\partial}{\partial \theta_{1}} \Delta_{\mathbf{k}}\left(\mathbf{v}_{\mathbf{2}}\right)=-\beta_{2}\left(\cos \vartheta \sin \theta_{1}-\sin \vartheta \cos \theta_{1} \cos \theta_{2}\right) \Delta_{\mathbf{k}}^{2}\left(\mathbf{v}_{\mathbf{2}}\right) .
\end{aligned}
$$

Using

$$
\frac{\mathrm{d}}{\mathrm{d} \theta_{1}} \frac{\sin ^{2-2 \varepsilon} \theta_{1}}{2-2 \varepsilon}=\sin ^{1-2 \varepsilon} \theta_{1} \cos \theta_{1}
$$

and

$$
1 \equiv\left(1-\beta_{1} \cos \theta_{1}\right) \Delta_{\mathbf{k}}\left(\mathbf{v}_{\mathbf{1}}\right)
$$

it holds

$$
\begin{aligned}
I_{j, l} & =\int \mathrm{d} \Omega_{k_{1} k_{2}} \Delta_{\mathbf{k}}^{j}\left(\mathbf{v}_{\mathbf{1}}\right) \Delta_{\mathbf{k}}^{l}\left(\mathbf{v}_{\mathbf{2}}\right) \\
& =I_{j+1, l}+\frac{\beta_{1}}{2-2 \varepsilon} \int_{0}^{\pi} \mathrm{d} \theta_{2} \sin ^{-2 \varepsilon} \theta_{2} \int_{0}^{\pi} \mathrm{d} \theta_{1} \sin ^{2-2 \varepsilon} \theta_{1} \frac{\partial}{\partial \theta_{1}}\left[\Delta_{\mathbf{k}}^{j+1}\left(\mathbf{v}_{\mathbf{1}}\right) \Delta_{\mathbf{k}}^{l}\left(\mathbf{v}_{\mathbf{2}}\right)\right] .
\end{aligned}
$$

Making the partial derivative

$$
\begin{aligned}
\beta_{1} \sin \theta_{1} \frac{\partial}{\partial \theta_{1}}\left[\Delta_{\mathbf{k}}^{j+1}\left(\mathbf{v}_{\mathbf{1}}\right) \Delta_{\mathbf{k}}^{l}\left(\mathbf{v}_{\mathbf{2}}\right)\right]= & l \Delta_{\mathbf{k}}^{j+1}\left(\mathbf{v}_{\mathbf{1}}\right) \Delta_{\mathbf{k}}^{l+1}\left(\mathbf{v}_{\mathbf{2}}\right)\left[L_{\mathbf{k}}\left(\mathbf{v}_{\mathbf{1}}\right) L_{\mathbf{k}}\left(\mathbf{v}_{\mathbf{2}}\right)-\beta_{1} \beta_{2} \cos \vartheta\right] \\
& +(j+1) \Delta_{\mathbf{k}}^{j+2}\left(\mathbf{v}_{\mathbf{1}}\right) \Delta_{\mathbf{k}}^{l}\left(\mathbf{v}_{\mathbf{2}}\right)\left[L_{\mathbf{k}}^{2}\left(\mathbf{v}_{\mathbf{1}}\right)-\beta_{1}^{2}\right]
\end{aligned}
$$

and plugging it into eq. (3.132) yields

$$
\begin{aligned}
I_{j, l}=I_{j+1, l}+\frac{1}{2-2 \varepsilon} & {\left[(j+1)\left(\left(1-\beta_{1}^{2}\right) I_{j+2, l}-2 I_{j+1, l}+I_{j, l}\right)\right.} \\
& \left.+l\left(\left(1-\beta_{1} \beta_{2} \cos \vartheta\right) I_{j+1, l+1}-I_{j, l+1}-I_{j+1, l}+I_{j, l}\right)\right] .
\end{aligned}
$$


Collecting $I_{j, l}$ with same indices and using $1-\beta_{1}^{2}=v_{11}$ and $1-\beta_{1} \beta_{2} \cos \vartheta=v_{12}$ results in the partial integration identity

$$
\begin{aligned}
0= & (j+l-1+2 \varepsilon) I_{j, l}-(2 j+l+2 \varepsilon) I_{j+1, l}+v_{11}(j+1) I_{j+2, l} \\
& -l I_{j, l+1}+l v_{12} I_{j+1, l+1} .
\end{aligned}
$$

Interchanging $\mathbf{v}_{\mathbf{1}}$ and $\mathbf{v}_{\mathbf{2}}$ yields the second complementary relation

$$
\begin{aligned}
0= & (j+l-1+2 \varepsilon) I_{j, l}-(2 l+j+2 \varepsilon) I_{j, l+1}+v_{22}(l+1) I_{j, l+2} \\
& -j I_{j+1, l}+j v_{12} I_{j+1, l+1} .
\end{aligned}
$$

\subsubsection{Recursion relations}

In section 3.3.2 and 3.3.3 we derived three important relations (3.105)-(3.136) based on differential and integration properties of angular integrals:

$$
\begin{aligned}
0= & (j-l)\left(1-v_{12}\right) I_{j, l}-j\left(1-v_{11}\right) I_{j+1, l-1}+l\left(1-v_{22}\right) I_{j-1, l+1} \\
& +j\left(v_{12}-v_{11}\right) I_{j+1, l}-l\left(v_{12}-v_{22}\right) I_{j, l+1}, \\
0= & (j+l-1+2 \varepsilon) I_{j, l}-(2 j+l+2 \varepsilon) I_{j+1, l}+v_{11}(j+1) I_{j+2, l} \\
& -l I_{j, l+1}+l v_{12} I_{j+1, l+1}, \\
0= & (j+l-1+2 \varepsilon) I_{j, l}-(2 l+j+2 \varepsilon) I_{j, l+1}+v_{22}(l+1) I_{j, l+2} \\
& -j I_{j+1, l}+j v_{12} I_{j+1, l+1} .
\end{aligned}
$$

The first relation is symmetric under exchange $\left(j, v_{11}\right) \leftrightarrow\left(l, v_{22}\right)$, while the second and the third are equivalent under such exchange. Using these relations one can establish recursion relations to deduce all angular integrals $I_{j, l}$ with $j, l \in \mathbb{Z}$. As starting point of the recursion procedure we use the set of three basic integrals: with no denominators $I_{0,0}=I^{(0)}=2 \pi /(1-2 \varepsilon)$, with one massive denominator $I_{1,0}=I_{1,0}^{(1)}\left(v_{11} ; \varepsilon\right)$, and with two massive denominators $I_{1,1}=I_{1,1}^{(2)}\left(v_{12}, v_{11}, v_{22}\right)$.

We are now in the position to provide the complete set of recursive relations involving angular integrals $I_{j, l}$ for all $j, l \in \mathbb{Z}$. We consider five cases depending on signs of the indices $j$ and $l$.

First case: $\boldsymbol{l}=\mathbf{0}$ and $\boldsymbol{j}>\mathbf{0}$ (or $\boldsymbol{j}=\mathbf{0}$ and $\boldsymbol{l}>\mathbf{0}$ ). We start with trivial case, when one of the indices is zero and the other is positive. In case of $l=0$ and $j>0$ we take the identity (3.138) put $l=0$ and shift index $j$ as $j \rightarrow j-2$. We get the required recursion relation

$$
I_{j, 0}=\frac{1}{v_{11}(j-1)}\left[2(j-2+\varepsilon) I_{j-1,0}-(j-3+2 \varepsilon) I_{j-2,0}\right],
$$

valid for $j \geq 2$. The starting input for the recursion are two basic (parent) integrals $I_{0,0}$ and $I_{1,0}$ corresponding to $j=0$ and $j=1$, respectively. 
Next three integrals for this case are determined by the relations:

$$
\begin{aligned}
I_{2,0} & =\frac{1}{v_{11}}\left[2 \varepsilon I_{1,0}+(1-2 \varepsilon) I_{0,0}\right], \\
I_{3,0} & =\frac{1}{v_{11}}\left[(1+\varepsilon) I_{2,0}-\varepsilon I_{1,0}\right], \\
I_{4,0} & =\frac{1}{3 v_{11}}\left[2(2+\varepsilon) I_{3,0}-(1+2 \varepsilon) I_{2,0}\right] .
\end{aligned}
$$

The recursion procedure for $j=0, l>0$ is completely analogous with $\left(j, v_{11}\right)$ and $\left(l, v_{22}\right)$ interchanged.

For massless case $v_{11}=0$ at $l=0$ and $j>0$ the recursion is again derived from identity (3.138) by shifting index $j$ as $j \rightarrow j-1$ :

$$
I_{j, 0}=\frac{j-2+2 \varepsilon}{2 j-2+2 \varepsilon} I_{j-1,0},
$$

valid for $j \geq 1$ and is starting with basic integral $I_{0,0}$.

In this case the next four integrals are fixed from:

$$
\begin{aligned}
& I_{1,0}=I_{0,0} \frac{-1+2 \varepsilon}{2 \varepsilon}, \\
& I_{2,0}=I_{1,0} \frac{\varepsilon}{1+\varepsilon}, \\
& I_{3,0}=I_{2,0} \frac{1+2 \varepsilon}{4+2 \varepsilon}, \\
& I_{4,0}=I_{3,0} \frac{1+\varepsilon}{3+\varepsilon} .
\end{aligned}
$$

The recursion for $j=0, l>0$ is completely analogous with $j$ and $l$ interchanged.

In appendix $\mathrm{G}$ we present explicit results for the angular integrals and perform the $\varepsilon$ expansion.

Second case: $\boldsymbol{l}=\mathbf{0}$ and $\boldsymbol{j}<\mathbf{0}$ (or $\boldsymbol{j}=\mathbf{0}$ and $\boldsymbol{l}<0$ ). In case $l=0$ and $j<0$ we again use the identity (3.138) and resort the terms there to get the following recursion relation:

$$
I_{j, 0}=\frac{1}{1-j-2 \varepsilon}\left[-2(j+\varepsilon) I_{j+1,0}+v_{11}(j+1) I_{j+2,0}\right],
$$

starting with $I_{0,0}$ and valid for $j \leq-1$. In particular, the next four integrals are:

$$
\begin{aligned}
& I_{-1,0} \equiv I_{0,0}, \\
& I_{-2,0}=I_{0,0} \frac{4-v_{11}-2 \varepsilon}{3-2 \varepsilon}, \\
& I_{-3,0}=\frac{1}{2-\varepsilon}\left[-v_{11} I_{0,0}+(3-\varepsilon) I_{-2,0}\right], \\
& I_{-4,0}=\frac{1}{5-2 \varepsilon}\left[-3 v_{11} I_{-2,0}+2(4-\varepsilon) I_{-3,0}\right] .
\end{aligned}
$$

The recursion for $j=0, l<0$ is completely analogous with $\left(j, v_{11}\right)$ and $\left(l, v_{22}\right)$ interchanged. 
In the massless case $\left(j<0, l=0, v_{11}=0\right)$ the recursion relation is further simplified to

$$
I_{j, 0}=-\frac{2(j+\varepsilon)}{1-j-2 \varepsilon} I_{j+1,0}
$$

In particular, the results for the next three integrals read

$$
\begin{aligned}
& I_{-2,0}=I_{-1,0} \frac{4-2 \varepsilon}{3-2 \varepsilon}, \\
& I_{-3,0}=I_{-2,0} \frac{3-\varepsilon}{2-\varepsilon}, \\
& I_{-4,0}=I_{-3,0} \frac{8-2 \varepsilon}{5-2 \varepsilon} .
\end{aligned}
$$

The case $\left(j=0, l<0, v_{22}=0\right)$ is completely analogous with $j$ and $l$ interchanged.

Third case: $\boldsymbol{j}<\mathbf{0}$ and $\boldsymbol{l}<\mathbf{0}$. In the case of no actual denominators $(j<0$ and $l<0)$ we can give a closed polynomial formula for $I_{j, l}$. Using eqs. (3.31), (3.43), (D.36), and (D.37) we get

$$
\begin{aligned}
I_{j, l}\left(v_{12}, v_{11}, v_{22} ; \varepsilon\right) \stackrel{j, l \leq 0}{=} & \pi \sum_{m=0}^{-j}\left(\begin{array}{c}
-j \\
m
\end{array}\right)\left(1-\sqrt{1-v_{11}}\right)^{-j-m}\left(1-v_{11}\right)^{m / 2} \\
& \times \sum_{n=0}^{-l}\left(\begin{array}{c}
-l \\
n
\end{array}\right)\left(1-\sqrt{1-v_{22}}\right)^{-l-n}\left(1-v_{22}\right)^{n / 2} \\
& \times 2^{m+n+1} \frac{(1-\varepsilon)_{m}(1-\varepsilon)_{n}}{(1-2 \varepsilon)_{m+n+1}} F_{1}\left(-m,-n, 1-\varepsilon, 1-\frac{\hat{v}_{12}}{2}\right) \\
= & \pi \sum_{m=0}^{-j} \sum_{n=0}^{-l} \sum_{p=0}^{\min (m, n)}\left(\begin{array}{c}
-j \\
m
\end{array}\right)\left(\begin{array}{c}
-l \\
n
\end{array}\right)\left(1-\sqrt{1-v_{11}}\right)^{-j-m}\left(1-v_{11}\right)^{m / 2} \\
& \times\left(1-\sqrt{1-v_{22}}\right)^{-l-n}\left(1-v_{22}\right)^{n / 2} 2^{m+n+1} \frac{(1-\varepsilon)_{m}(1-\varepsilon)_{n}}{(1-2 \varepsilon)_{m+n+1}} \\
& \times \frac{(-m)_{p}(-n)_{p}}{(1-\varepsilon)_{p} p !}\left(1-\frac{\hat{v}_{12}}{2}\right)^{p},
\end{aligned}
$$

where

$$
\hat{v}_{12}=\frac{1}{2}\left(1+\frac{1-v_{12}}{\sqrt{1-v_{11}} \sqrt{1-v_{22}}}\right) .
$$

Here the sum over $p$ terminates at $\min (m, n)$, because $(-m)_{p}(-n)_{p}=0$ at $p>\min (m, n)$.

To complement the exact result for both negative indices we derive the recursion relation. It is derived summing two basic formulas (3.138) and (3.139). One gets:

$$
\begin{aligned}
I_{j, l}= & \frac{1}{2(j+l-1+2 \varepsilon)}\left[(3 l+j+2 \varepsilon) I_{j, l+1}+(3 j+l+2 \varepsilon) I_{j+1, l}\right. \\
& \left.-v_{11}(j+1) I_{j+2, l}-v_{22}(l+1) I_{j, l+2}-v_{12}(j+l) I_{j+1, l+1}\right] .
\end{aligned}
$$


This recursion relation is valid for all $j, l \leq-1$ and manifestly symmetric under interchange of two sets $\left(j, v_{11}\right)$ and $\left(l, v_{22}\right)$. E.g., the expressions for determination of a few first integrals read:

$$
\begin{aligned}
I_{-1,-1} & =\frac{1}{3-2 \varepsilon}\left[(2-\varepsilon)\left(I_{-1,0}+I_{0,-1}\right)-v_{12} I_{0,0}\right]=I_{0,0} \frac{4-2 \varepsilon-v_{12}}{3-2 \varepsilon}, \\
I_{-1,-2} & =\frac{1}{4(2-\varepsilon)}\left[(7-2 \varepsilon) I_{-1,-1}+(5-2 \varepsilon) I_{0,-2}-v_{22} I_{-1,0}-3 v_{12} I_{0,-1}\right] \\
& =I_{0,0} \frac{6-2 \varepsilon-2 v_{12}-v_{22}}{3-2 \varepsilon}, \\
I_{-2,-2} & =\frac{1}{5-2 \varepsilon}\left[(4-\varepsilon)\left(I_{-2,-1}+I_{-1,-2}\right)-\frac{v_{11}}{2} I_{0,-2}-\frac{v_{22}}{2} I_{-2,0}-2 v_{12} I_{-1,-1}\right] \\
& =\frac{I_{0,0}}{(3-2 \varepsilon)(5-2 \varepsilon)}\left[(3-\varepsilon)\left(4-\varepsilon-\frac{v_{11}+v_{22}+4 v_{22}}{2}\right)+v_{11} v_{22}+2 v_{12}^{2}\right] .
\end{aligned}
$$

In appendix $\mathrm{G}$ we display the integrals for $-4 \leq j, l \leq-1$ and perform their $\varepsilon$ expansion up to order $\varepsilon$. Note, that the limit of massless particles $v_{11}=0$ or/and $v_{22}=0$ is straightforward in this case.

Fourth case: $j>0$ and $l<0$ (or $l>0, j<0$ ). For the case, that the indices of the angular integral have opposite signs $j>0$ and $l<0$ (or $l>0, j<0$ ) we involve all three master identities (3.137)-(3.139). In particular, in case $j>0$ and $l<0$ we do the following: (1) first, we shift indices $j \rightarrow j-1$ in (3.138) and $l \rightarrow l-1$ in (3.139); (2) sum three equations multiplying each of them with $-1,-\left(1-v_{12}\right)$, and $\left(1-v_{11}\right)$, respectively. After shifting index $l \rightarrow l+1$ in the resulting expression we get the recursive relation

$$
\begin{aligned}
I_{j, l}= & \frac{1-v_{12}}{1-v_{11}} I_{j-1, l+1}+\frac{1}{\left(1-v_{11}\right)(j+l-1+2 \varepsilon)}\left[I_{j-1, l+2}(l+1)\left(v_{12}-v_{22}\right)\right. \\
& \left.-I_{j, l+2}(l+1) X+I_{j, l+1}(j+2 l+2 \varepsilon)\left(v_{12}-v_{11}\right)\right]
\end{aligned}
$$

where $X=v_{12}^{2}-v_{11} v_{22}$. Starting integrals for this recursion, which are known to us, are $I_{0,0}, I_{1,0}$, and $I_{0,1}$. The integral $I_{1,1}$ does not appear in this recursion since $(l+1) I_{j, l+2}=0$ at $l=-1$.

We present a few examples of the expressions for the angular integrals with $j>0$ and $l<0$ :

$$
\begin{aligned}
I_{1,-1}= & \frac{1-v_{12}}{1-v_{11}} I_{0,0}+\frac{v_{12}-v_{11}}{1-v_{11}} I_{1,0}, \\
I_{1,-2}= & \frac{1-v_{12}}{1-v_{11}} I_{0,-1}+\frac{1}{2\left(1-v_{11}\right)(1-\varepsilon)}\left[\left(v_{12}-v_{22}\right) I_{0,0}-X I_{1,0}\right. \\
& \left.+\left(v_{12}-v_{11}\right)(3-2 \varepsilon) I_{1,-1}\right], \\
I_{2,-2}= & \frac{1-v_{12}}{1-v_{11}} I_{1,-1}+\frac{1}{\left(1-v_{11}\right)(1-2 \varepsilon)}\left[\left(v_{12}-v_{22}\right) I_{1,0}-X I_{2,0}\right. \\
& \left.+2\left(v_{12}-v_{11}\right)(1-\varepsilon) I_{2,-1}\right] .
\end{aligned}
$$


By analogy we can derive the recursive relation for the case with $j<0$ and $l>0$ making interchange of two sets $\left(j, v_{11}\right)$ and $\left(l, v_{22}\right)$ :

$$
\begin{aligned}
I_{j, l}= & \frac{1-v_{12}}{1-v_{22}} I_{j+1, l-1}+\frac{1}{\left(1-v_{22}\right)(j+l-1+2 \varepsilon)}\left[I_{j+2, l-1}(j+1)\left(v_{12}-v_{11}\right)\right. \\
& \left.-I_{j+2, l}(j+1) X+I_{j+1, l}(2 j+l+2 \varepsilon)\left(v_{12}-v_{22}\right)\right] .
\end{aligned}
$$

Fifth case: $\boldsymbol{j}>\mathbf{0}$ and $\boldsymbol{l}>\mathbf{0}$. To derive the recursive relations for the $j>0$ and $l>0$ case we start with eqs. (3.152), (3.154) and shift the indices there as $l \rightarrow l-2$ in eq. (3.152) and $j \rightarrow j-2$ in eq. (3.154). We get two recursive relations

$$
\begin{aligned}
I_{j, l}= & \frac{1}{X(j-1)}\left[I_{j-1, l}\left(v_{12}-v_{22}\right)(2 j+l-4+2 \varepsilon)-I_{j-2, l}\left(1-v_{22}\right)(j+l-3+2 \varepsilon)\right. \\
& \left.+I_{j, l-1}(j-1)\left(v_{12}-v_{11}\right)+I_{j-1, l-1}(j+l-3+2 \varepsilon)\left(1-v_{12}\right)\right] \\
I_{j, l}= & \frac{1}{X(l-1)}\left[I_{j, l-1}\left(v_{12}-v_{11}\right)(2 l+j-4+2 \varepsilon)-I_{j, l-2}\left(1-v_{11}\right)(j+l-3+2 \varepsilon)\right. \\
& \left.+I_{j-1, l}(l-1)\left(v_{12}-v_{22}\right)+I_{j-1, l-1}(j+l-3+2 \varepsilon)\left(1-v_{12}\right)\right]
\end{aligned}
$$

valid for $j \geq 2, l \geq 1$ and $j \geq 1, l \geq 2$ and starting with basic integral $I_{1,1}$. One can see that eq. (3.155) is not valid for $j=1, l=2$, while eq. (3.156) is not valid for $j=2, l=1$ because of a pole. To avoid the pole and to get the recursive relation, which is manifestly symmetric under interchange of $\left(j, v_{11}\right)$ and $\left(l, v_{22}\right)$ we simply multiply eq. (3.155) with factor $X(j-1)$ and eq. (3.156) with factor $X(l-1)$ and sum them up. As a result we get the recursive relation, which is more suitable for our purposes

$$
\begin{aligned}
I_{j, l}= & \frac{j+l-3+2 \varepsilon}{X(j+l-2)}\left[2\left(1-v_{12}\right) I_{j-1, l-1}-\left(1-v_{11}\right) I_{j, l-2}-\left(1-v_{22}\right) I_{j-2, l}\right] \\
& +\frac{2 j+2 l-5+2 \varepsilon}{X(j+l-2)}\left[\left(v_{12}-v_{11}\right) I_{j, l-1}+\left(v_{12}-v_{22}\right) I_{j-1, l}\right]
\end{aligned}
$$

valid for all $j, l \geq 1$ and $j+l \neq 2$. Note, that $j+l \neq 2$ means that $j=l=1$ and this choice corresponds to the master integral $I_{1,1}$.

Below we list few examples of integrals with positive $j$ and $l$ :

$$
\begin{aligned}
I_{1,2}= & \frac{1}{X}\left[\left(v_{12}-v_{11}\right)(1+2 \varepsilon) I_{1,1}-2\left(1-v_{11}\right) \varepsilon I_{1,0}\right. \\
& \left.+2\left(1-v_{12}\right) \varepsilon I_{0,1}+\left(v_{12}-v_{22}\right) I_{0,2}\right], \\
I_{2,1}= & \frac{1}{X}\left[\left(v_{12}-v_{22}\right)(1+2 \varepsilon) I_{1,1}-2\left(1-v_{22}\right) \varepsilon I_{0,1}\right. \\
& \left.+2\left(1-v_{12}\right) \varepsilon I_{1,0}+\left(v_{12}-v_{11}\right) I_{2,0}\right], \\
I_{2,2}= & \frac{1+2 \varepsilon}{2 X}\left[2\left(1-v_{12}\right) I_{1,1}-\left(1-v_{11}\right) I_{2,0}-\left(1-v_{22}\right) I_{0,2}\right] \\
& +\frac{3+2 \varepsilon}{2 X}\left[\left(v_{12}-v_{11}\right) I_{2,1}+\left(v_{12}-v_{22}\right) I_{1,2}\right] .
\end{aligned}
$$


Finally, we stress that the main result of this section is the derivation of the set of recursive relations [see eqs. (3.140), (3.142), (3.144), (3.146), (3.150), (3.152), (3.154)(3.157)], which together with three master integrals $I_{0,0}, I_{1,0}$, and $I_{1,1}$ can deduce any angular $I_{j, l}$ with arbitrary indices $j$ and $l$. Therefore, the $\varepsilon$-expansion of any $I_{j, l}$ follows from expansion of master integrals $I_{0,0}, I_{1,0}$, and $I_{1,1}$. The recursion relations have been implemented together with the all-order expansions of the basis integrals in Mathematica. This allows for fast calculation of any $I_{j, l}$ with $j, l \in \mathbb{Z}$ to arbitrary order in $\varepsilon$.

\subsection{All order $\varepsilon$-expansion of angular integrals}

The hypergeometric representations of the angular integrals derived in section 3.2 allow for a systematic $\varepsilon$-expansion up to desired order. In the cases where the angular integrals are expressible in terms of the Gauss hypergeometric function there are publicly available algorithms for the $\varepsilon$-expansion up to considerably high order. A well-suited Mathematicabased package is HypExp [82], which provides analytic expansions in terms of harmonic polylogarithms.

In this section we derive the analytical formalism allowing for the all order $\varepsilon$-expansions for the integrals up to two denominators (i.e. the basic angular integrals) in terms of the single and double Nielsen polylogarithms $[83,84]$. As it was shown in section 3.2.6 for arbitrary dimension $D$ the double massive angular integral can be transformed to a finite sum of single-massive integrals based on the reduction formalism proposed by us. Therefore, $\varepsilon$-expansion of the double massive integral for any $j, l \in \mathbb{Z}$ can be generated by use of the $\varepsilon$-expansion of the single massive integral. Using two mass splitting (see section 3.2.6) and recursion relations (see section 3.3.4) any angular integral $I_{j, l}\left(v_{12}, v_{11}, v_{22} ; \varepsilon\right)$ with $j, l \in \mathbb{Z}$ can be expressed in terms of the basic angular integrals mentioned above. Note, that these results have never been discussed or derived before in literature. Only expansion up to a few first orders in $\varepsilon$ were known before (see, e.g. results in refs. [44, 48, 65]). Therefore, we think that our findings will be useful as input for the calculation of QCD processes.

\subsubsection{Massive integral with one denominator}

We start with the hypergeometric representation for the massive integral with one denominator (3.30)

$$
\begin{aligned}
I_{1}^{(1)}\left(v_{11} ; \varepsilon\right) & =\frac{I^{(0)}(\varepsilon)}{(1-\beta)}{ }_{2} F_{1}\left(1,1-\varepsilon, 2-2 \varepsilon,-\frac{2 \beta}{1-\beta}\right) \\
& =\frac{2 \pi}{1-2 \varepsilon} \frac{1}{1-\sqrt{1-v_{11}}}{ }_{2} F_{1}\left(1,1-\varepsilon, 2-2 \varepsilon, 1-\frac{1+\sqrt{1-v_{11}}}{1-\sqrt{1-v_{11}}}\right) .
\end{aligned}
$$

The $\varepsilon$-expansion of the ${ }_{2} F_{1}(1,1-\varepsilon, 2-2 \varepsilon, x)$ is established the following way. First, we perform the Euler transformation and employ the Euler integral representation for the Gauss hypergeometric function ${ }_{2} F_{1}$ :

$$
\begin{aligned}
{ }_{2} F_{1}(1,1-\varepsilon, 2-2 \varepsilon, x) & =(1-x)^{-\varepsilon} F_{2} F_{1}(1-\varepsilon, 1-2 \varepsilon, 2-2 \varepsilon, x) \\
& =(1-x)^{-\varepsilon}(1-2 \varepsilon) \int_{0}^{1} \mathrm{~d} t t^{-2 \varepsilon}(1-x t)^{-1+\varepsilon} .
\end{aligned}
$$


Next using $\frac{\partial}{\partial x}(1-x t)^{\varepsilon}=-\varepsilon t(1-x t)^{-1+\varepsilon}$ we get

$$
{ }_{2} F_{1}(1,1-\varepsilon, 2-2 \varepsilon, x)=-\frac{1-2 \varepsilon}{\varepsilon}(1-x)^{-\varepsilon} \frac{\partial}{\partial x} \int_{0}^{1} \frac{\mathrm{d} t}{t} t^{-2 \varepsilon}(1-x t)^{\varepsilon} .
$$

To separate the pole at $t=0$ in eq. (3.160) we use the decomposition

$$
(1-x t)^{\varepsilon}=1+\left[(1-x t)^{\varepsilon}-1\right]
$$

and drop the first constant term, since it vanishes when differentiated w.r.t. $x$. Then we perform the $\varepsilon$ expansion of the remaining $(1-x t)^{\varepsilon}-1$. It yields

$$
\begin{aligned}
{ }_{2} F_{1}(1,1-\varepsilon, 2-2 \varepsilon, x)= & -\frac{1-2 \varepsilon}{\varepsilon}(1-x)^{-\varepsilon} \sum_{n=0}^{\infty} \sum_{m=1}^{\infty} \frac{(-2)^{n}}{n ! m !} \varepsilon^{m+n} \\
& \times \frac{\partial}{\partial x} \int_{0}^{1} \frac{\mathrm{d} t}{t} \log ^{n} t \log ^{m}(1-x t) .
\end{aligned}
$$

The latter expansion can be written in a more compact form using the familiar Nielsen polylogarithms [83, 84]

$$
S_{n, p}(x) \equiv \frac{(-1)^{n+p-1}}{(n-1) ! p !} \int_{0}^{1} \frac{\mathrm{d} t}{t} \log ^{n-1} t \log ^{p}(1-x t),
$$

which satisfies the differential equation

$$
\frac{\partial}{\partial x} S_{n, p}(x)=\frac{1}{x} S_{n-1, p}(x) .
$$

Hence, we have

$$
\begin{aligned}
{ }_{2} F_{1}(1,1-\varepsilon, 2-2 \varepsilon, x) & =-\frac{1-2 \varepsilon}{\varepsilon}(1-x)^{-\varepsilon} \sum_{n=0}^{\infty} \sum_{m=1}^{\infty} 2^{n}(-1)^{m} \varepsilon^{m+n} \frac{\partial}{\partial x} S_{n+1, m}(x) \\
& =-\frac{1-2 \varepsilon}{\varepsilon} \frac{(1-x)^{-\varepsilon}}{x} \sum_{n=0}^{\infty} \sum_{m=1}^{\infty} 2^{n}(-1)^{m} \varepsilon^{m+n} S_{n, m}(x) .
\end{aligned}
$$

Next, rearranging the summation to make it over $N=m+n-1$ and shifting $m \rightarrow m+1$ yields

$$
{ }_{2} F_{1}(1,1-\varepsilon, 2-2 \varepsilon, x)=(1-2 \varepsilon) \frac{(1-x)^{-\varepsilon}}{x} \sum_{N=0}^{\infty} \sum_{m=0}^{N} 2^{N-m}(-1)^{m} S_{N-m, m+1}(x) \varepsilon^{N} .
$$

Therefore, we have established the all order $\varepsilon$-expansion of the massive integral with one denominator (3.30)

$$
\begin{aligned}
I_{1}^{(1)}\left(v_{11} ; \varepsilon\right)= & \frac{\pi}{\sqrt{1-v_{11}}}\left(\frac{1+\sqrt{1-v_{11}}}{1-\sqrt{1-v_{11}}}\right)^{-\varepsilon} \\
& \times \sum_{N=0}^{\infty} \sum_{m=0}^{N} 2^{N-m}(-1)^{m+1} S_{N-m, m+1}\left(1-\frac{1+\sqrt{1-v_{11}}}{1-\sqrt{1-v_{11}}}\right) \varepsilon^{N}
\end{aligned}
$$


Using specific Nielsen polylogarithms $S_{0, p}(x)=(-1)^{p} \log ^{p}(1-x) / p$ ! and $S_{n, 1}(x)=\operatorname{Li}_{n+1}(x)$ this explicitly gives up to order $\varepsilon$

$$
\begin{aligned}
I_{1}^{(1)}\left(v_{11} ; \varepsilon\right)= & \frac{\pi}{\sqrt{1-v_{11}}}\left(\frac{1+\sqrt{1-v_{11}}}{1-\sqrt{1-v_{11}}}\right)^{-\varepsilon}\left[\log \left(\frac{1+\sqrt{1-v_{11}}}{1-\sqrt{1-v_{11}}}\right)\right. \\
& \left.+\frac{\varepsilon}{2} \log ^{2}\left(\frac{1+\sqrt{1-v_{11}}}{1-\sqrt{1-v_{11}}}\right)-2 \varepsilon \operatorname{Li}_{2}\left(1-\frac{1+\sqrt{1-v_{11}}}{1-\sqrt{1-v_{11}}}\right)+\mathcal{O}\left(\varepsilon^{2}\right)\right] .
\end{aligned}
$$

The latter formula is exactly the same expansion as obtained in ref. [48].

Eq. (3.168) together with the recursion relation (3.140) completes all order $\varepsilon$-expansion of the angular integrals $I_{j}^{(1)}\left(v_{11} ; \varepsilon\right)$ with one massive denominator. In particular, starting from $I_{0}^{(1)}\left(v_{11} ; \varepsilon\right) \equiv I^{(0)}(\varepsilon)=2 \pi /(1-2 \varepsilon)$ and $I_{1}^{(1)}\left(v_{11} ; \varepsilon\right)$ given by eq. (3.168) we can derive analytical results for any power of massive denominator $j$. For example, the results for $j=2,3,4$ are given by the recursion identities:

$$
\begin{aligned}
& I_{2}^{(1)}\left(v_{11} ; \varepsilon\right)=\frac{1}{v_{11}}\left[2 \varepsilon I_{1}^{(1)}\left(v_{11} ; \varepsilon\right)+(1-2 \varepsilon) I^{(0)}(\varepsilon)\right], \\
& I_{3}^{(1)}\left(v_{11} ; \varepsilon\right)=\frac{1}{2 v_{11}}\left[2(1+\varepsilon) I_{2}^{(1)}\left(v_{11} ; \varepsilon\right)-2 \varepsilon I_{1}^{(1)}\left(v_{11} ; \varepsilon\right)\right], \\
& I_{4}^{(1)}\left(v_{11} ; \varepsilon\right)=\frac{1}{3 v_{11}}\left[2(2+\varepsilon) I_{3}^{(1)}\left(v_{11} ; \varepsilon\right)-(1+2 \varepsilon) I_{2}^{(2)}\left(v_{11} ; \varepsilon\right)\right] .
\end{aligned}
$$

\subsubsection{Massless integral with two denominators}

For the two denominator massless integral we start with the hypergeometric representation $(3.43)$

$$
\begin{aligned}
I_{1,1}^{(0)}\left(v_{12} ; \varepsilon\right) & =I^{(0)}(\varepsilon) \frac{(-2 \varepsilon)_{2}}{2^{2}(-\varepsilon)_{1}(-\varepsilon)_{1}}{ }_{2} F_{1}\left(1,1,1-\varepsilon, 1-\frac{v_{12}}{2}\right) \\
& =-\frac{\pi}{\varepsilon}{ }_{2} F_{1}\left(1,1,1-\varepsilon, 1-\frac{v_{12}}{2}\right) .
\end{aligned}
$$

The $\varepsilon$-expansion of the ${ }_{2} F_{1}(1,1,1-\varepsilon, x)$ runs analogous to the one denominator one mass case considered above. First, we perform the Euler transformation and employ the Euler integral representation of the ${ }_{2} F_{1}$ :

$$
\begin{aligned}
{ }_{2} F_{1}(1,1,1-\varepsilon, x) & =(1-x)^{-1-\varepsilon}{ }_{2} F_{1}(-\varepsilon,-\varepsilon, 1-\varepsilon, x) \\
& =(1-x)^{-1-\varepsilon}(-\varepsilon) \int_{0}^{1} \mathrm{~d} t t^{-\varepsilon-1}(1-x t)^{\varepsilon} .
\end{aligned}
$$

The pole at $t=0$ is taken care of by subtracting the numerator at the pole. Afterwards we can safely expand in $\varepsilon$.

$$
\begin{aligned}
{ }_{2} F_{1}(1,1,1-\varepsilon, x) & =-\varepsilon(1-x)^{-1-\varepsilon}\left(\int_{0}^{1} \frac{\mathrm{d} t}{t} t^{-\varepsilon}\left[(1-x t)^{\varepsilon}-1\right]+\int_{0}^{1} \mathrm{~d} t t^{-\varepsilon-1}\right) \\
& =-\varepsilon(1-x)^{-1-\varepsilon}\left(\int_{0}^{1} \frac{\mathrm{d} t}{t} \sum_{n=0}^{\infty} \frac{(-\varepsilon)^{n}}{n !} \log ^{n} t \sum_{m=1}^{\infty} \frac{\varepsilon^{m}}{m !} \log ^{m}(1-x t)-\frac{1}{\varepsilon}\right) .
\end{aligned}
$$


Rearranging the terms and evaluating the logarithmic integrals in terms of the Nielsen polylogarithms yields

$$
\begin{aligned}
{ }_{2} F_{1}(1,1,1-\varepsilon, x) & =\varepsilon(1-x)^{-1-\varepsilon}\left[1-\varepsilon \sum_{n=0}^{\infty} \sum_{m=1}^{\infty} \frac{(-1)^{n}}{n ! m !} \varepsilon^{m+n} \int_{0}^{1} \frac{\mathrm{d} t}{t} \log ^{n} t \log ^{m}(1-x t)\right] \\
& =\varepsilon(1-x)^{-1-\varepsilon}\left[1-\varepsilon \sum_{n=0}^{\infty} \sum_{m=1}^{\infty}(-1)^{m} \varepsilon^{m+n} S_{n+1, m}(x)\right] .
\end{aligned}
$$

Performing the summation over $N=m+n+1$ and $m$ gives

$$
{ }_{2} F_{1}(1,1,1-\varepsilon, x)=(1-x)^{-1-\varepsilon}\left[1-\sum_{N=2}^{\infty} \sum_{m=1}^{N-1}(-1)^{m} S_{N-m, m}(x) \varepsilon^{N}\right] .
$$

Therefore we have established the all order expansion

$$
I_{1,1}^{(0)}\left(v_{12}, \varepsilon\right)=\pi\left(\frac{v_{12}}{2}\right)^{-1-\varepsilon}\left[-\frac{1}{\varepsilon}+\sum_{N=1}^{\infty} \sum_{m=1}^{N}(-1)^{m} S_{N-m+1, m}\left(1-\frac{v_{12}}{2}\right) \varepsilon^{N}\right] .
$$

For checking purposes we explicitly write out the terms up to order $\varepsilon$ and confirm that it exactly coincides with result derived before in refs. [44, 48, 65]:

$$
I_{1,1}^{(0)}\left(v_{12}, \varepsilon\right)=\pi\left(\frac{v_{12}}{2}\right)^{-1-\varepsilon}\left[-\frac{1}{\varepsilon}-\varepsilon \operatorname{Li}_{2}\left(1-\frac{v_{12}}{2}\right)+\mathcal{O}\left(\varepsilon^{2}\right)\right] .
$$

\subsubsection{Single massive integral with two denominators}

For the two denominator single massive integral we start with the hypergeometric representation (3.60)

$$
\begin{aligned}
I_{1,1}^{(1)}\left(v_{12}, v_{11} ; \varepsilon\right) & =\frac{I_{1}^{(0)}(\varepsilon)}{v_{12}}\left(\frac{v_{11}}{v_{12}^{2}}\right)^{\varepsilon} F_{1}\left(-2 \varepsilon,-\varepsilon,-\varepsilon, 1-2 \varepsilon, \omega_{+}, \omega_{-}\right) \\
\omega_{ \pm} & =\frac{\tau_{ \pm}}{1-\tau_{ \pm}}=1-\frac{v_{12}}{1 \pm \sqrt{1-v_{11}}},
\end{aligned}
$$

where $I_{1}^{(0)}(\varepsilon)=-\pi / \varepsilon$. The $\varepsilon$-expansion of the Appell function $F_{1}(-2 \varepsilon,-\varepsilon,-\varepsilon, 1-2 \varepsilon, x, y)$ is established similarly to the previous cases. Employing the Euler integral representation and separating the pole gives

$$
\begin{aligned}
F_{1}(-2 \varepsilon,-\varepsilon,-\varepsilon, 1-2 \varepsilon, x, y) & =-2 \varepsilon \int_{0}^{1} \mathrm{~d} t t^{-1-2 \varepsilon}(1-x t)^{\varepsilon}(1-y t)^{\varepsilon} \\
& =-2 \varepsilon\left(\int_{0}^{1} \frac{\mathrm{d} t}{t} t^{-2 \varepsilon}\left[(1-x t)^{\varepsilon}(1-y t)^{\varepsilon}-1\right]+\int_{0}^{1} \mathrm{~d} t t^{-1-2 \varepsilon}\right) .
\end{aligned}
$$

Now we can safely expand in $\varepsilon$ under the integral to obtain

$$
\begin{aligned}
F_{1}(\ldots) & =-2 \varepsilon\left(\int_{0}^{1} \frac{\mathrm{d} t}{t} \sum_{n=0}^{\infty} \frac{(-2 \varepsilon)^{n}}{n !} \log ^{n} t \sum_{m=1}^{\infty} \frac{\varepsilon^{m}}{m !} \log ^{m}((1-x t)(1-y t))-\frac{1}{2 \varepsilon}\right) \\
& =1-2 \varepsilon \sum_{n=0}^{\infty} \sum_{m=1}^{\infty} \frac{(-2)^{n}}{n ! m !} \varepsilon^{m+n} \int_{0}^{1} \frac{\mathrm{d} t}{t} \log ^{n} t \log ^{m}((1-x t)(1-y t)) .
\end{aligned}
$$


By binomial expansion the $\log ^{m}$ term can be written in the form

$$
\begin{aligned}
\log ^{m}((1-x t)(1-y t)) & =(\log (1-x t)+\log (1-y t))^{m} \\
& =\sum_{k=0}^{m}\left(\begin{array}{c}
m \\
k
\end{array}\right) \log ^{m-k}(1-x t) \log ^{k}(1-y t) .
\end{aligned}
$$

Hence we can write

$$
F_{1}(\ldots)=1-2 \varepsilon \sum_{n=0}^{\infty} \sum_{m=1}^{\infty} \frac{(-2)^{n}}{n ! m !} \varepsilon^{m+n} \sum_{k=0}^{m}\left(\begin{array}{c}
m \\
k
\end{array}\right) \int_{0}^{1} \frac{\mathrm{d} t}{t} \log ^{n} t \log ^{m-k}(1-x t) \log ^{k}(1-y t)
$$

The logarithmic integral is the natural two-variable generalization of the Nielsen polylogarithm. Defining the double Nielsen polylogarithm [see discussion of their properties in appendix F]

$$
S_{n, p_{1}, p_{2}}(x, y) \equiv \frac{(-1)^{n+p_{1}+p_{2}-1}}{(n-1) ! p_{1} ! p_{2} !} \int_{0}^{1} \frac{\mathrm{d} t}{t} \log ^{n-1} t \log ^{p_{1}}(1-x t) \log ^{p_{2}}(1-y t)
$$

we can evaluate the integrals to

$$
F_{1}(\ldots)=1-2 \varepsilon \sum_{n=0}^{\infty} \sum_{m=1}^{\infty}(-1)^{m} 2^{n} \varepsilon^{m+n} \sum_{k=0}^{m} S_{n+1, m-k, k}(x, y) .
$$

Changing summation to run over $N=m+n+1$ and $m$ yields the result

$$
F_{1}(-2 \varepsilon,-\varepsilon,-\varepsilon, 1-2 \varepsilon, x, y)=1-\sum_{N=2}^{\infty} \sum_{m=1}^{N-1}(-1)^{m} 2^{N-m} \sum_{k=0}^{m} S_{N-m, m-k, k}(x, y) \varepsilon^{N} .
$$

This establishes the all-order expansion of the single massive two denominator integral in the form

$$
\begin{aligned}
I_{1,1}^{(1)}\left(v_{12}, v_{11}, \varepsilon\right)= & \frac{\pi}{v_{12}}\left(\frac{v_{11}}{v_{12}^{2}}\right)^{\varepsilon}\left[-\frac{1}{\varepsilon}\right. \\
& \left.+\sum_{N=1}^{\infty} \sum_{m=1}^{N}(-1)^{m} 2^{N-m+1} \sum_{k=0}^{m} S_{N-m+1, m-k, k}\left(\tau_{+}, \tau_{-}\right) \varepsilon^{N}\right] .
\end{aligned}
$$

Up to order $\varepsilon$ this expression is

$$
\begin{aligned}
I_{1,1}^{(1)}\left(v_{12}, v_{11}, \varepsilon\right)= & \frac{\pi}{v_{12}}\left(\frac{v_{11}}{v_{12}^{2}}\right)^{\varepsilon}\left[-\frac{1}{\varepsilon}-2 \varepsilon\left(\operatorname{Li}_{2}\left(1-\frac{v_{12}}{1+\sqrt{1-v_{11}}}\right)\right.\right. \\
& \left.\left.+\operatorname{Li}_{2}\left(1-\frac{v_{12}}{1-\sqrt{1-v_{11}}}\right)\right)+\mathcal{O}\left(\varepsilon^{2}\right)\right] .
\end{aligned}
$$

Genuine double Nielsen polylogarithms start appearing at order $\varepsilon^{2}$. 


\subsubsection{Double massive integral with two denominators}

As we showed in section 3.2.6 the double massive integral reduces to a sum of single massive integrals using two mass splitting [see details in eqs. (3.64) and (3.64)]. In particular, there are two fully equivalent decompositions of the double mass integral governed by the mixing parameter $\lambda$. Due to rotational invariance both solutions for the $\lambda$ parameter and correspondingly for the scalar products of the velocities $v_{13}$ and $v_{23}$ are equivalent to each other. Hence, we restrict to one of them, e.g. to

$$
\begin{aligned}
\lambda & =\frac{v_{11}-v_{12}-\sqrt{X}}{v_{11}+v_{22}-2 v_{12}}, \\
v_{13} & =\frac{v_{11}\left(v_{22}+\sqrt{X}\right)-v_{12}\left(v_{12}+\sqrt{X}\right)}{v_{11}+v_{22}-2 v_{12}}, \\
v_{23} & =\frac{v_{22}\left(v_{11}-\sqrt{X}\right)-v_{12}\left(v_{12}-\sqrt{X}\right)}{v_{11}+v_{22}-2 v_{12}},
\end{aligned}
$$

where $X=v_{12}^{2}-v_{11} v_{22}$.

In this case the expression for the double massive integral $I_{1,1}^{(2)}\left(v_{12}, v_{11}, v_{22} ; \varepsilon\right)$ reads:

$$
I_{1,1}^{(2)}\left(v_{12}, v_{11}, v_{22} ; \varepsilon\right)=\frac{1}{\sqrt{X}}\left[v_{13} I_{1,1}^{(1)}\left(v_{13}, v_{11} ; \varepsilon\right)-v_{23} I_{1,1}^{(1)}\left(v_{23}, v_{22} ; \varepsilon\right)\right] .
$$

Here we used the relations $\lambda=v_{13} / \sqrt{X}$ and $1-\lambda=-v_{23} / \sqrt{X}$. After cancelling the pole and some rearrangement one gets

$$
\begin{aligned}
I_{1,1}^{(2)}\left(v_{12}, v_{11}, v_{22} ; \varepsilon\right)= & \frac{\pi}{\sqrt{X}}\left[\log \left(\frac{v_{12}+\sqrt{X}}{v_{12}-\sqrt{X}}\right)-\sum_{n=1}^{\infty} \frac{\varepsilon^{n}}{(n+1) !}\left(\log ^{n+1} \frac{v_{11}}{v_{13}^{2}}-\log ^{n+1} \frac{v_{22}}{v_{23}^{2}}\right)\right. \\
& +\left(\frac{v_{11}}{v_{13}^{2}}\right)^{\varepsilon} \sum_{N=1}^{\infty} \sum_{m=1}^{N}(-1)^{m} 2^{N-m+1} \sum_{k=0}^{m} S_{N-m+1, m-k, k}\left(\tau_{+}^{13}, \tau_{-}^{13}\right) \varepsilon^{N} \\
& \left.-\left(\frac{v_{22}}{v_{23}^{2}}\right)^{\varepsilon} \sum_{N=1}^{\infty} \sum_{m=1}^{N}(-1)^{m} 2^{N-m+1} \sum_{k=0}^{m} S_{N-m+1, m-k, k}\left(\tau_{+}^{23}, \tau_{-}^{23}\right) \varepsilon^{N}\right],
\end{aligned}
$$

with $\tau_{ \pm}^{13}=1-v_{13} /\left(1 \pm \sqrt{1-v_{11}}\right)$ and $\tau_{ \pm}^{23}=1-v_{23} /\left(1 \pm \sqrt{1-v_{11}}\right)$. In derivation of eq. (3.192) we use the identity:

$$
\log \frac{v_{22}}{v_{23}^{2}}-\log \frac{v_{11}}{v_{13}^{2}}=\log \left(\frac{v_{12}+\sqrt{X}}{v_{12}-\sqrt{X}}\right) .
$$

Explicitly, the $\varepsilon$ expansion up to second order reads:

$$
\begin{aligned}
I_{1,1}^{(2)}\left(v_{12}, v_{11}, v_{22} ; \varepsilon\right)=\frac{1}{\sqrt{X}} & {\left[v_{13} I_{1,1}^{(1)}\left(v_{13}, v_{11} ; \varepsilon\right)-v_{23} I_{1,1}^{(1)}\left(v_{23}, v_{22} ; \varepsilon\right)\right] } \\
=\frac{\pi}{\sqrt{X}} & {\left[\log \left(\frac{v_{12}+\sqrt{X}}{v_{12}-\sqrt{X}}\right)-\varepsilon\left(\frac{1}{2} \log ^{2} \frac{v_{11}}{v_{13}^{2}}-\frac{1}{2} \log ^{2} \frac{v_{22}}{v_{23}^{2}}\right.\right.} \\
& +2 \operatorname{Li}_{2}\left(1-\frac{v_{13}}{1-\sqrt{1-v_{11}}}\right)+2 \operatorname{Li}_{2}\left(1-\frac{v_{13}}{1+\sqrt{1-v_{11}}}\right) \\
& \left.\left.-2 \operatorname{Li}_{2}\left(1-\frac{v_{23}}{1-\sqrt{1-v_{22}}}\right)-2 \operatorname{Li}_{2}\left(1-\frac{v_{23}}{1+\sqrt{1-v_{22}}}\right)\right)\right] \\
+ & \mathcal{O}\left(\varepsilon^{2}\right) .
\end{aligned}
$$




\subsection{Neerven integrals}

In this section we would like to discuss some aspects of the Neerven angular integrals:

1. connection between the Neerven $[44,65]$ and Somogyi [48] parametrization of angular integrals;

2. some important remarks on massless and double massive Neerven integrals;

3. comment on Mirkes's recursive formula [49] for a special class of single massive Neerven integrals.

\subsubsection{Connection between Neerven and Somogyi parametrization}

Discussion of angular integrals in the literature revolves for the most part around results for integrals of the form

$$
I_{D}^{(j, l)}(a, b, A, B, C)=\int \mathrm{d} \Omega_{k_{1} k_{2}} \frac{1}{\left(a+b \cos \theta_{1}\right)^{j}\left(A+B \cos \theta_{1}+C \sin \theta_{1} \cos \theta_{2}\right)^{l}} .
$$

In this section we will review how these angular integrals presented in the Neerven form can be translated to those in the Somogyi form

$$
\int \mathrm{d} \Omega_{k_{1} k_{2}} \frac{1}{\left(v_{1} \cdot k\right)^{j}\left(v_{2} \cdot k\right)^{l}},
$$

introduced in section 3.2. Defining

$$
v_{1}=\left(1, \mathbf{0}_{D-3}, 0,-\frac{b}{a}\right), \quad v_{2}=\left(1, \mathbf{0}_{D-3},-\frac{C}{A},-\frac{B}{A}\right),
$$

we have

$$
v_{11}=1-\frac{b^{2}}{a^{2}}, \quad v_{22}=1-\frac{B^{2}+C^{2}}{A^{2}}, \quad v_{12}=1-\frac{b B}{a A} .
$$

With $k=\left(1, \ldots, \sin \theta_{1} \cos \theta_{2}, \cos \theta_{1}\right)$ we can write the generic Neerven integral as

$$
I_{D}^{(j, l)}(a, b, A, B, C)=\frac{1}{a^{j} A^{l}} \int \mathrm{d} \Omega_{k_{1} k_{2}} \frac{1}{\left(v_{1} \cdot k\right)^{j}\left(v_{2} \cdot k\right)^{l}} .
$$

For the four classes of the Neerven integrals this implies

Massless case: $a^{2}=b^{2}$ and $A^{2}=B^{2}+C^{2}$.

$$
I_{4-2 \varepsilon}^{(j, l)}(a, b, A, B, C)=\frac{1}{a^{j} A^{l}} I_{j, l}^{(0)}\left(1-\frac{b B}{a A} ; \varepsilon\right) \text {. }
$$

Single massive case: $a^{2}=b^{2}$.

$$
I_{4-2 \varepsilon}^{(j, l)}(a, b, A, B, C)=\frac{1}{a^{j} A^{l}} I_{j, l}^{(1)}\left(1-\frac{b B}{a A}, 1-\frac{B^{2}+C^{2}}{A^{2}} ; \varepsilon\right) .
$$

Single massive case: $A^{2}=B^{2}+C^{2}$.

$$
I_{4-2 \varepsilon}^{(j, l)}(a, b, A, B, C)=\frac{1}{a^{j} A^{l}} I_{j, l}^{(1)}\left(1-\frac{b B}{a A}, 1-\frac{b^{2}}{a^{2}} ; \varepsilon\right) .
$$


Double massive case: $a^{2} \neq b^{2}$ and $A^{2} \neq B^{2}+C^{2}$.

$$
I_{4-2 \varepsilon}^{(j, l)}(a, b, A, B, C)=\frac{1}{a^{j} A^{l}} I_{j, l}^{(2)}\left(1-\frac{b B}{a A}, 1-\frac{b^{2}}{a^{2}}, 1-\frac{B^{2}+C^{2}}{A^{2}} ; \varepsilon\right) .
$$

Note, that there are the following limits:

1. $I_{j, l}^{(2)}$ reduces to $I_{j, l}^{(1)}$ at $v_{11}=0$ or $v_{22}=0$,

2. $I_{j, l}^{(1)}$ reduces to $I_{j, l}^{(0)}$ at $v_{11}=0$.

Therefore, the integral $I_{j, l}^{(2)}$ can be regarded as the basic Neerven integral (1.1) encoding information about the others. Applying the result from section 3.3.1 we can write down the general Neerven integral in hypergeometric form in terms of the Lauricella function $F_{B}^{(3)}$ :

$$
\begin{aligned}
I_{4-2 \varepsilon}^{(j, l)}(a, b, A, B, C)= & \frac{1}{a^{j} A^{l}} \frac{2 \pi}{1-2 \varepsilon}\left(1-\frac{b B}{a A}\right)^{1-j-l-\varepsilon} \\
& \times F_{B}^{(3)}\left(\frac{j}{2}, \frac{l}{2}, \frac{3-j-l-2 \varepsilon}{2}, \frac{j+1}{2}, \frac{l+1}{2}, \frac{2-j-l-2 \varepsilon}{2}, \frac{3}{2}-\varepsilon ; x, y, z\right),
\end{aligned}
$$

with the abbreviations $x=\frac{b(A b-a B)}{a(a A-b B)}, y=\frac{a\left(B^{2}+C^{2}\right)-A b B}{A(a A-b B)}$, and $z=\frac{b B}{a A}$.

For $j, l \in \mathbb{N}_{>0}$ we also derive the closed formula for the angular integral using results from section 3.2 in terms of the Appell function $F_{1}$

$$
\begin{aligned}
& I_{4-2 \varepsilon}^{(j, l)}(a, b, A, B, C)=\frac{1}{a^{j} A^{l}} \frac{2 \pi}{1-2 \varepsilon} \\
& \quad \times\left[\sum_{n=0}^{j-1}\left(\begin{array}{c}
l-1+n \\
l-1
\end{array}\right) \frac{\lambda_{ \pm}^{l}\left(1-\lambda_{ \pm}\right)^{n}(2-l-n-2 \varepsilon)_{l+n}}{\left(v_{13}^{ \pm}\right)^{j-n} 2^{l+n}(1-l-n-\varepsilon)_{l+n}}\left(\frac{v_{11}}{v_{13}^{ \pm 2}}\right)^{l+n-1+\varepsilon}\right. \\
& \quad \times F_{1}\left(2-j-l-2 \varepsilon, 1-l-n-\varepsilon, 1-l-n-\varepsilon, 2-l-n-2 \varepsilon, x_{13}^{ \pm}, y_{13}^{ \pm}\right) \\
& \quad+\sum_{n=0}^{l-1}\left(\begin{array}{c}
j-1+n \\
j-1
\end{array}\right) \frac{\lambda_{ \pm}^{n}\left(1-\lambda_{ \pm}\right)^{j}(2-l-n-2 \varepsilon)_{j+n}}{\left(v_{23}^{ \pm}\right)^{l-n} 2^{j+n}(1-j-n-\varepsilon)_{j+n}}\left(\frac{v_{22}}{v_{23}^{ \pm 2}}\right)^{j+n-1+\varepsilon} \\
& \left.\quad \times F_{1}\left(2-j-l-2 \varepsilon, 1-j-n-\varepsilon, 1-j-n-\varepsilon, 2-j-n-2 \varepsilon, x_{23}^{ \pm}, y_{23}^{ \pm}\right)\right],
\end{aligned}
$$

where the following notations are used:

$$
\begin{aligned}
& v_{11}=1-\frac{b^{2}}{a^{2}}, \quad v_{22}=1-\frac{B^{2}+C^{2}}{A^{2}}, \quad v_{12}=1-\frac{b B}{a A}, \\
& \lambda_{ \pm}=\frac{A\left(a-a b B+A b^{2} \pm \sqrt{(A b-a B)^{2}+C^{2}(a-b)(a+b)}\right)}{a^{2} C^{2}+(A b-a B)^{2}}, \\
& v_{13}^{ \pm}=\left(1-\lambda_{ \pm}\right) v_{11}+\lambda_{ \pm} v_{12}, \quad v_{23}^{ \pm}=\left(1-\lambda_{ \pm}\right) v_{12}+\lambda_{ \pm} v_{22}, \\
& x_{13}^{ \pm}=1-\frac{v_{13}^{ \pm}}{1+\sqrt{1-v_{11}}}, \quad y_{13}^{ \pm}=1-\frac{v_{13}^{ \pm}}{1-\sqrt{1-v_{11}}}, \\
& x_{23}^{ \pm}=1-\frac{v_{23}^{ \pm}}{1+\sqrt{1-v_{22}}}, \quad y_{23}^{ \pm}=1-\frac{v_{23}^{ \pm}}{1-\sqrt{1-v_{22}}} \text {. }
\end{aligned}
$$




\subsubsection{On massless integral in the Neerven parametrization}

For a check of consistency, we want to derive the massless Neerven integral in its most commonly used form. This concerns the integral (compare with ref. [44])

$$
\int \mathrm{d} \Omega_{k_{1} k_{2}} \frac{1}{\left(1-\cos \theta_{1}\right)^{j}\left(1-\cos \vartheta \cos \theta_{1}-\sin \vartheta \sin \theta_{1} \cos \theta_{2}\right)^{l}} .
$$

In the notation we have established, we write eq. (3.207) as

$$
I_{4-2 \varepsilon}^{(j, l)}(1,-1,1,-\cos \vartheta,-\sin \vartheta)=I_{j, l}^{(0)}(1-\cos \vartheta ; \varepsilon),
$$

employing the result (3.44) and rewriting the cosine with the half-angle formula we obtain

$$
I_{4-2 \varepsilon}^{(j, l)}(1,-1,1,-\cos \vartheta,-\sin \vartheta)=I_{j+l}^{(0)}(\varepsilon) \frac{(1-j-l-\varepsilon)_{j}}{(1-j-\varepsilon)_{j}}{ }_{2} F_{1}\left(j l, 1-\varepsilon, \cos ^{2} \frac{\vartheta}{2}\right) .
$$

Writing down $I_{j+l}^{(0)}(\varepsilon)$ and the Pochhammer symbols in terms of gamma functions we arrive at the well known result (see, e.g., ref. [63])

$$
\begin{aligned}
& \int \mathrm{d} \Omega_{k_{1} k_{2}} \frac{1}{\left(1-\cos \theta_{1}\right)^{j}\left(1-\cos \vartheta \cos \theta_{1}-\sin \vartheta \sin \theta_{1} \cos \theta_{2}\right)^{l}} \\
& \quad=2^{1-j-l} \pi \frac{\Gamma(1-2 \varepsilon)}{\Gamma^{2}(1-\varepsilon)} B(1-j-\varepsilon, 1-l-\varepsilon){ }_{2} F_{1}\left(j, l, 1-\varepsilon, \cos ^{2} \frac{\vartheta}{2}\right) .
\end{aligned}
$$

\subsubsection{On massive integral in the Neerven parametrization}

To derive the four dimensional result for the double massive integral the proposed method via two-mass splitting is of course unnecessarily involved. One can instead employ Weierstrass substitution $\xi=\tan (\theta / 2)$ on both integrals in eq. (1.1) and obtain, by taking advantage of the trigonometric identities

$$
\cos x=\frac{1-\tan ^{2} \frac{x}{2}}{1+\tan ^{2} \frac{x}{2}}, \quad \sin x=\frac{2 \tan \frac{x}{2}}{1+\tan ^{2} \frac{x}{2}},
$$

the integral representation

$$
\begin{aligned}
I_{4-2 \varepsilon}^{(j, l)}(a, b, A, B, C)= & 2^{3-4 \varepsilon} \int_{0}^{\infty} \mathrm{d} \xi_{1} \int_{0}^{\infty} \mathrm{d} \xi_{2} \\
& \times \frac{\left(1+\xi_{1}^{2}\right)^{j+l-2+2 \varepsilon}\left(1+\xi_{2}^{2}\right)^{l-1+2 \varepsilon} \xi_{1}^{1-2 \varepsilon} \xi_{2}^{-2 \varepsilon}}{\Delta^{j}\left(a, b, \xi_{1}\right) \Delta^{l}\left(A, B, C, \xi_{1}, \xi_{2}\right)},
\end{aligned}
$$

where

$$
\begin{aligned}
\Delta\left(a, b, \xi_{1}\right) & =a+b+(a-b) \xi_{1}^{2}, \\
\Delta\left(A, B, C, \xi_{1}, \xi_{2}\right) & =\left(A+B+(A-B) \xi_{1}^{2}\right)\left(1+\xi_{2}^{2}\right)+2 C \xi_{1}^{2}\left(1-\xi_{2}^{2}\right) .
\end{aligned}
$$

For $\varepsilon=0$ and $j=l=1$ this vastly simplifies and can be elementary integrated to yield (compare, e.g., with ref. [55])

$$
I_{4}^{(1,1)}(a, b, A, B, C)=\frac{\pi}{\sqrt{X}} \log \left(\frac{a A-b B+\sqrt{X}}{a A-b B-\sqrt{X}}\right),
$$


with $X=(a A-b B)^{2}-\left(A^{2}-B^{2}-C^{2}\right)\left(a^{2}-b^{2}\right)$. Any higher values $j, l \in \mathbb{N}$ can be reached by the differential identities (see also ref. [66])

$$
I_{D}^{(j+1, l)}=-\frac{1}{j} \frac{\partial}{\partial a} I_{D}^{(j, l)}, \quad I_{D}^{(j, l+1)}=-\frac{1}{l} \frac{\partial}{\partial A} I_{D}^{(j, l+1)},
$$

the first requiring $a^{2} \neq b^{2}$ the second $A^{2} \neq B^{2}+C^{2}$.

\subsubsection{Recursive determination of Mirkes type III integrals}

In section 3.3 we developed the methods for the derivation of relations between angular integrals. One should stress that there have been attempts in this direction in the literature before. For example, in ref. [49] Mirkes made an effort to derive the recursive formula for a special kind of single-massive Neerven integrals. We find that his formula is correct only in four dimensions and here we discuss how to extend it to arbitrary dimension. The subject of study is the single massive angular integral

$$
I_{D}^{j, l}(A, B, C)=\int \mathrm{d} \Omega_{k_{1} k_{2}} \frac{\left(-\cos \theta_{1}\right)^{l}}{\left(A+B \cos \theta_{1}+C \sin \theta_{1} \cos \theta_{2}\right)^{j}}, \quad l \in \mathbb{N} .
$$

In order to derive the recursive relation for the integral (3.216) we write it down in the form of a double massive integral with two denominators and express it in terms, which occurred before in our calculations (see section 3.2.6):

$$
\begin{aligned}
I_{D}^{j, l}(A, B, C) & =\lim _{\beta \rightarrow \infty} I_{D}^{j, l}(A, B, C ; \beta), \text { with } \\
I_{D}^{j, l}(A, B, C ; \beta) & =\int \mathrm{d} \Omega_{k_{1} k_{2}} \frac{\left(\frac{1}{\beta}-\cos \theta_{1}\right)^{l}}{\left(A+B \cos \theta_{1}+C \sin \theta_{1} \cos \theta_{2}\right)^{j}} \\
& =\frac{1}{A^{j} \beta^{l}} \int \mathrm{d} \Omega_{k_{1} k_{2}} \frac{\left(1-\beta \cos \theta_{1}\right)^{l}}{\left(1+\frac{B}{A} \cos \theta_{1}+\frac{C}{A} \sin \theta_{1} \cos \theta_{2}\right)^{j}},
\end{aligned}
$$

where the parameter $\beta$ should go to infinity after deriving the recursive relations. Here, the velocities of massive particles are chosen as

$$
v_{1}=\left(1, \mathbf{0}_{D-3},-\frac{C}{A},-\frac{B}{A}\right), \quad v_{2}=\left(1, \mathbf{0}_{D-2}, \beta\right)
$$

The scalar products of the velocities read

$$
\begin{aligned}
& v_{11}=v_{1}^{2}=1-\frac{B^{2}+C^{2}}{A^{2}}, \quad v_{22}=v_{2}^{2}=1-\beta^{2}, \quad v_{12}=v_{1} \cdot v_{2}=1+\beta \frac{B}{A}, \\
& X=v_{12}^{2}-v_{11} v_{22}=\frac{(A \beta+B)^{2}+C^{2}\left(1-\beta^{2}\right)}{A^{2}}
\end{aligned}
$$

Using notations from (3.219) the integral $I_{D}^{j, l}(A, B, C ; \beta)$ can be classified as a double massive integral with two denominators (with positive $j$ and negative $-l$ powers):

$$
I_{D}^{j, l}(A, B, C ; \beta)=\frac{1}{A^{j} \beta^{l}} I_{j,-l}^{(2)}\left(v_{12}, v_{11}, v_{22}\right)
$$


Next we obtain the recursion relation for $I_{D}^{j, l}(A, B, C ; \beta)$ using the 4 th type recursion relation (3.152) derived for the integral $I_{j, l}^{(2)}\left(v_{12}, v_{11}, v_{22}\right)$ at $j>0$ and $-l<0$. We have:

$$
\begin{aligned}
I_{D}^{j, l}(A, B, C ; \beta)= & \frac{1}{B^{2}+C^{2}}\left[-B I_{D}^{j-1, l-1}(A, B, C ; \beta)\right. \\
& +\frac{1-l}{j-l-1+2 \varepsilon}\left(A+\frac{B}{\beta}\right) I_{D}^{j-1, l-2}(A, B, C ; \beta) \\
& -\frac{1-l}{j-l-1+2 \varepsilon}\left(A^{2}-C^{2}+\frac{2 A B}{\beta}+\frac{B^{2}+C^{2}}{\beta^{2}}\right) I_{D}^{j, l-2}(A, B, C ; \beta) \\
& \left.+\frac{j-2 l+2 \varepsilon}{j-l-1+2 \varepsilon}\left(A B-\frac{A^{4}}{\beta\left(B^{2}+C^{2}\right)}\right) I_{D}^{j, l-1}(A, B, C ; \beta)\right]
\end{aligned}
$$

Finally taking the limit $\beta \rightarrow \infty$ we derive the recursion relation for type III integrals for arbitrary dimension and valid for $l \geq 1$ and $j>0$ :

$$
\begin{aligned}
I_{D}^{j, l}(A, B, C)= & \frac{1}{B^{2}+C^{2}}\left[-B I_{D}^{j-1, l-1}(A, B, C)\right. \\
& +\frac{1-l}{j-l-1+2 \varepsilon}\left(A I_{D}^{j-1, l-2}(A, B, C)-\left(A^{2}-C^{2}\right) I_{D}^{j, l-2}(A, B, C)\right) \\
& \left.+\frac{j-2 l+2 \varepsilon}{j-l-1+2 \varepsilon} A B I_{D}^{j, l-1}(A, B, C)\right] .
\end{aligned}
$$

This is the main result of this section. Note, that in specific cases $j=1$ and $j=2$ the recursions relation read

$$
\begin{aligned}
I_{D}^{1, l}(A, B, C)= & \frac{1}{l\left(B^{2}+C^{2}\right)}\left[\frac{1+(-1)^{l}}{2} \frac{l}{l-2 \varepsilon} \frac{\left(\frac{3}{2}\right)_{\frac{l}{2}-1}}{\left(\frac{3}{2}-\varepsilon\right)_{\frac{l}{2}-1}} A I^{(0)}\right. \\
& -\frac{1-(-1)^{l}}{2} \frac{\left(\frac{3}{2}\right)_{\frac{l-1}{2}}}{\left(\frac{3}{2}-\varepsilon\right)_{\frac{l-1}{2}}} B I^{(0)}+\frac{l(1-l)}{l-2 \varepsilon}\left(A^{2}-C^{2}\right) I_{D}^{1, l-2}(A, B, C) \\
& \left.-\frac{l(1-2 l+2 \varepsilon)}{l-2 \varepsilon} A B I_{D}^{1, l-1}(A, B, C)\right] \\
I_{D}^{2, l}(A, B, C)= & \frac{\partial}{\partial A} I_{D}^{1, l}(A, B, C)=-\frac{1}{l\left(B^{2}+C^{2}\right)}\left[\frac{1+(-1)^{l}}{2} \frac{l}{l-2 \varepsilon} \frac{\left(\frac{3}{2}\right)_{\frac{l}{2}-1}}{\left(\frac{3}{2}-\varepsilon\right)_{\frac{l}{2}-1}} I^{(0)}\right. \\
& +\frac{l(1-l)}{l-2 \varepsilon}\left(2 A I_{D}^{1, l-2}(A, B, C)-\left(A^{2}-C^{2}\right) I_{D}^{2, l-2}(A, B, C)\right)_{(3.22)} \\
& -\frac{l(1-2 l+2 \varepsilon)}{l-2 \varepsilon} B\left(I_{D}^{1, l-1}(A, B, C ; \beta)-A I_{D}^{2, l-1}(A, B, C ; \beta)\right)
\end{aligned}
$$


To derive eqs. (3.223) and (3.224), we used

$$
I_{D}^{0, l}=\int \mathrm{d} \Omega_{k_{1} k_{2}}\left(-\cos \theta_{1}\right)^{l}=\frac{1+(-1)^{l}}{2(1+l)} \frac{\left(\frac{3}{2}\right)_{\frac{l}{2}}}{\left(\frac{3}{2}-\varepsilon\right)_{\frac{l}{2}}} I^{(0)} .
$$

In the limit $\varepsilon=0$ (i.e. $D=4$ ) we exactly reproduce the recursion relations (D.23) and (D.24) in ref. [49]. The latter are valid only at $\varepsilon=0$ and fail for $\varepsilon \neq 0$.

In case of $l=0$ and $j \geq 2$ we use the recursion relation (3.140), which is in notations of type III integrals reads:

$$
\begin{aligned}
I_{D}^{j, 0}(A, B, C)= & \frac{1}{A v_{11}(j-1)}\left[2(j-2+\varepsilon) I_{D}^{j-1,0}(A, B, C)\right. \\
& \left.-\frac{1}{A}(j-3+2 \varepsilon) I_{D}^{j-2,0}(A, B, C)\right],
\end{aligned}
$$

where $v_{11}$ is defined in eq. (3.219). As before, the recursion (3.226) starts with master integrals $I_{D}^{0,0}(A, B, C)=I^{(0)}$ and $I_{D}^{1,0}(A, B, C)=I_{1}^{(1)} / A$. In appendix G.6 using eqs. (3.223)(3.224) we present analytical results for the integrals for $j=1,2$ and $l=0, \ldots, 4$.

By analogy with integrals (3.227) one can pin down the similar integrals

$$
J_{D}^{j, l}(A, B, C)=\int \mathrm{d} \Omega_{k_{1} k_{2}} \frac{\left(-\sin \theta_{1} \cos \theta_{2}\right)^{l}}{\left(A+B \cos \theta_{1}+C \sin \theta_{1} \cos \theta_{2}\right)^{j}} .
$$

It is clear that in this case we can do the same trick - introducing the massive propagator $\left(1 / \beta-\sin \theta_{1} \cos \theta_{2}\right)^{l}$ with $\beta \rightarrow \infty$ and choose the second velocity $v_{2}$ and therefore the scalar product $v_{12}$ as $v_{2}=\left(1, \mathbf{0}_{D-3}, \beta, 0\right)$ and $v_{12}=1+\beta \frac{C}{A}$. One can see that the two similar types of integrals $I_{D}^{j, l}(A, B, C)$ and $J_{D}^{j, l}(A, B, C)$ are related upon interchange of two arguments $B \leftrightarrow C$ as

$$
J_{D}^{j, l}(A, B, C) \equiv I_{D}^{j, l}(A, C, B) .
$$

\section{Conclusions}

In present paper we discussed and developed new ideas and methods for calculation of loop and angular integrals in $D=4-2 \varepsilon$ dimensions in QCD, which serve as input for the study of perturbative matrix elements relevant for different processes such as Drell-Yan, SIDIS, etc. In particular, our paper contains two main topics - handling of loop and angular integrals. In the first case we further develop the Pasarino-Veltman (PV) technique [3] for dealing with tensorial integrals. We proposed a covariant formalism for consideration of loop integrals with rich Lorentz structure (so-called tensorial loop integrals) arising from decomposition of Feynman matrix elements. The method is based on the use of the orthogonal basis [see eq. (2.3)], which is formed by specific linear combinations of external momenta, which is quite simple and convenient for making analytical and numerical calculations. Note that our choice of the orthogonal basis is by construction free of soft singularities occurring in the limit of vanishing momenta. Also it gives the straightforward results for the scalar 
functions in the expansion of tensor loop integrals, i.e. without solving a system of equations like in the original PV method. All scalar functions are manifestly Lorentz covariant and depend on the set of Mandelstam variables. In present paper we restricted ourselves to the consideration of diagrams up to four external legs, but an extension of our method to more complicated diagrams (e.g., with 5 or 6 legs) is straightforward and we commented on it. All scalar functions are related via recurrence relations and finally presented in terms of PV functions or in terms of hypergeometric functions.

In the second part of our paper we presented a detailed and systematic analysis of angular integrals, which play an important role in the study of angular structure of QCD observables at next-to-leading order in the expansion in strong coupling constant. In particular, it is important to have complete and analytical set of these integrals for massive and massless particles and perform their $\varepsilon$ expansion up to desired order. Based on methods developed before in refs. [44-49] we implement new ideas for consistent and analytical treatment of angular integral with arbitrary number of propagators and independent of their power and kinematical properties. We explicitly discuss our method for splitting of the product of arbitrary number of propagators allowing to reduce it to the product of one or two propagators in appendix D. Also we studied in detail the integro-differential properties of angular integrals in order to derive partial differential and integral identities and the recursion relations. For the first time in literature we derive a complete set of recursion relations, which allow to deduce any angular integral with two denominators in arbitrary power and with arbitrary on-shell properties. We consider all particular cases:

1. the powers of the propagators are of the same sign and positive;

2. the powers of the propagators are of the same sign and negative;

3. the powers of the propagators have opposite signs;

4. the power of one denominator is simply zero.

Next important issue we considered is the systematic $\varepsilon$ expansion of angular integrals up to desired order. We demonstrated that this task can be solved for all types of integrals (i.e. for any powers of denominators and for any kinematical situations). For some integrals, e.g., with negative powers for both propagators we derived a closed polynomial formula for the angular integral. For other cases, based on the recursion relations it is enough to perform the $\varepsilon$-expansion of the three master integrals $I_{0,0}, I_{1,0}$, and $I_{1,1}$. We developed a Mathematica package to reduce angular integrals to master integrals and to perform the symbolic all-order $\varepsilon$ expansion. This allows for fast calculation of any $I_{j, l}$ with arbitrary $j, l \in \mathbb{Z}$ and up to arbitrary order in $\varepsilon$ expansion. All technical details of our consideration of loop and angular integrals are placed in the appendices, including tabulated integrals with specific on-shell properties and powers of the denominators.

Finally, we summarize our main results:

1. We developed new formalism for reduction of tensor loop integrals in $D$ dimensions based on the PV technique [3]. Our formalism allows to expand matrix elements 
through a set of Lorentz structures induced by the basis of orthogonal linear combinations of external momenta.

2. We discovered new properties of angular integrals: partial fraction decomposition (reduction of a number of denominators in the integrand); integro-differential identities leading to derivation of recursion relations between angular integrals with arbitrary indices; hypergeometric representation for all two-denominators angular integrals including double-massive case was derived.

3. We formulated approach for all order $\varepsilon$-expansion of angular integrals, which is based on expansion of the set of three parent integrals, while the expansion of the others is performed with the use of recursion relations. The recursion starts with the parent integrals.

\section{Acknowledgments}

We thank Werner Vogelsang for stimulating and useful discussions. This work was funded by BMBF "Verbundprojekt 05P2018 - Ausbau von ALICE am LHC: Jets und partonische Struktur von Kernen" (Förderkennzeichen: 05P18VTCA1), by ANID PIA/APOYO AFB180002 (Chile) and by FONDECYT (Chile) under Grant No. 1191103. This study was supported by Deutsche Forschungsgemeinschaft (DFG) through the Research Unit FOR 2926 "Next Generation pQCD for Hadron Structure: Preparing for the EIC" (Project number 40824754).

\section{A Triangle integrals}

Here, we present analytical results for the scalar functions occurring in the expansion of the triangle vector and tensorial (rank-2) loop integrals.

\section{A.1 Scalar and vector triangle integrals}

First, we discuss calculation of scalar triangle integral $C_{0}\left(s_{1}, s_{2}\right)$ with two massive external legs. Here, we follow derivation done in ref. [24]. We start with the integral

$$
C_{0}\left(s_{1}, s_{2}\right)=\left(i \pi^{2}\right)^{-1} \mu^{4-D} \int d^{D} k \frac{1}{k^{2}\left(k+l_{1}\right)^{2}\left(k+l_{2}\right)^{2}},
$$

where $s_{1}=l_{1}^{2}, s_{2}=l_{2}^{2}, s_{3}=\left(l_{1}-l_{2}\right)^{2}=0$. Note that for $s_{3} \neq 0$ the $C_{0}\left(s_{1}, s_{2}, s_{3}\right)$ is fully symmetric under permutation of the arguments $s_{1}, s_{2}$, and $s_{3}$. Therefore, result, which will be obtained for $s_{3}=0$ and $s_{1}, s_{2} \neq 0$ is trivially extended to other two cases of one vanishing mass: $s_{1}=0, s_{2}, s_{3} \neq 0$, and $s_{2}=0, s_{1}, s_{3} \neq 0$.

After $\alpha$ parametization and integration over loop momentum, the integral $C_{0}\left(s_{1}, s_{2}\right)$ takes the form

$$
C_{0}\left(s_{1}, s_{2}\right)=-\left(\pi \mu^{-2}\right)^{\frac{D}{2}-2} \Gamma\left(3-\frac{D}{2}\right) \int_{0}^{\infty} d \alpha_{1} \ldots \int_{0}^{\infty} d \alpha_{3} \frac{\delta\left(1-\sum_{i=1}^{3} \alpha_{i}\right)\left(\sum_{i=1}^{3} \alpha_{i}\right)^{3-D}}{\left(-s_{1} \alpha_{1} \alpha_{3}-s_{2} \alpha_{2} \alpha_{3}\right)^{3-\frac{D}{2}}} .
$$


Then we apply the change of variables ( $\alpha$ parameters) using the trick from [24]:

$$
\alpha_{1}=\eta_{1} \xi, \quad \alpha_{2}=\eta_{1}(1-\xi), \quad \alpha_{3}=\eta_{2} .
$$

Subsequently we take into account that the Jacobian of such the change of variables is $J=\eta_{1}$ and integrate over $\eta_{2}$ using $\delta$ function.

One gets:

$$
C_{0}\left(s_{1}, s_{2}\right)=-\left(\pi \mu^{-2}\right)^{\frac{D}{2}-2} \Gamma\left(3-\frac{D}{2}\right) \int_{0}^{1} d \xi \int_{0}^{1} d \eta \frac{\eta^{\frac{D}{2}-2}(1-\eta)^{\frac{D}{2}-3}}{\left(-s_{1} \xi-s_{2}(1-\xi)\right)^{3-\frac{D}{2}}} .
$$

Next we integrate over $\eta$ using the integral representation for the beta function

$$
B(m, n)=\frac{\Gamma(m) \Gamma(n)}{\Gamma(m+n)}=\int_{0}^{1} d \eta \eta^{m-1}(1-\eta)^{n-1},
$$

where $\Gamma(m)$ is the gamma function. Integration over $\xi$ proceeds using simple table integral

$$
\int_{0}^{1} \frac{d \xi}{(\xi+A)^{n+1}}=\frac{A^{-n}-(1+A)^{-n}}{n} .
$$

After these tricks the scalar triangle integral takes the form

$$
C_{0}\left(s_{1}, s_{2}\right)=\pi^{\frac{D}{2}-2} \frac{2 \Gamma\left(2-\frac{D}{2}\right) \Gamma^{2}\left(\frac{D}{2}-1\right)}{(4-D) \Gamma(D-4)} \frac{\left(-\frac{s_{1}}{\mu^{2}}\right)^{\frac{D}{2}-2}-\left(-\frac{s_{2}}{\mu^{2}}\right)^{\frac{D}{2}-2}}{s_{1}-s_{2}} .
$$

Next using the definition of the scalar bubble function $B_{0}(s)(2.7)$ we express $C_{0}\left(s_{1}, s_{2}\right)$ in terms of $B_{0}(s)$ functions as:

$$
C_{0}\left(s_{1}, s_{2}\right)=\frac{2(D-3)}{4-D} \frac{B_{0}\left(s_{1}\right)-B_{0}\left(s_{2}\right)}{s_{1}-s_{2}},
$$

which is manifestly symmetric under change of variables $s_{1}$ and $s_{2}$. For the $C_{0}$ function depending on one argument we introduce the following notation

$$
C_{0}(s)=C_{0}(s, 0)=C_{0}(0, s) .
$$

Now we show results for the vector integrals:

$$
C_{1}^{\mu ; 0 i j}=\left(i \pi^{2}\right)^{-1} \mu^{4-D} \int d^{D} k \frac{k^{\mu}}{\Delta_{0} \Delta_{i} \Delta_{j}}=P^{\mu} C_{1 P}^{0 i j}+R^{\mu} C_{1 R}^{0 i j}+T^{\mu} C_{1 T}^{0 i j} .
$$

All scalar functions $C_{1 P}^{0 i j}, C_{1 R}^{0 i j}$, and $C_{1 T}^{0 i j}$ are expressed in terms of scalar function (2.27) manifestly symmetric under $s_{1} \leftrightarrow s_{2}$ permutation:

$$
C_{1}\left(s_{1}, s_{2}, s_{3}\right)=\frac{s_{1}+s_{2}-s_{3}}{\lambda\left(s_{1}, s_{2}, s_{3}\right)}\left[B_{0}\left(s_{1}\right)-B_{0}\left(s_{3}\right)-s_{2} C_{0}\left(s_{1}, s_{2}, s_{3}\right)\right] .
$$


For specific limits it reduces to:

Limit I $\left(s_{3}=0\right)$

$$
\begin{aligned}
C_{1}\left(s_{1}, s_{2}, 0\right) & =\frac{s_{1}+s_{2}}{\left(s_{1}-s_{2}\right)^{2}}\left[B_{0}\left(s_{1}\right)-B_{0}(0)-s_{2} C_{0}\left(s_{1}, s_{2}\right)\right] \\
& =\frac{s_{1}+s_{2}}{\left(s_{1}-s_{2}\right)^{2}}\left[\frac{4-D}{2(D-3)} s_{1} C_{0}\left(s_{1}\right)-s_{2} C_{0}\left(s_{1}, s_{2}\right)\right] .
\end{aligned}
$$

Limit II $\left(s_{1}=0\right)$

$$
\begin{aligned}
C_{1}\left(0, s_{2}, s_{3}\right) & =\frac{1}{s_{2}-s_{3}}\left[B_{0}(0)-B_{0}\left(s_{3}\right)-s_{2} C_{0}\left(s_{2}, s_{3}\right)\right] \\
& =-\frac{1}{s_{2}-s_{3}}\left[\frac{4-D}{2(D-3)} s_{3} C_{0}\left(s_{3}\right)+s_{2} C_{0}\left(s_{2}, s_{3}\right)\right] .
\end{aligned}
$$

Limit III $\left(s_{2}=0\right)$

$$
\begin{aligned}
C_{1}\left(s_{1}, 0, s_{3}\right) & =\frac{1}{s_{1}-s_{3}}\left[B_{0}\left(s_{1}\right)-B_{0}\left(s_{3}\right)\right] \\
& =\frac{4-D}{2(D-3)} C_{0}\left(s_{1}, s_{3}\right) .
\end{aligned}
$$

Limit IV $\left(s_{1}=s_{3}=0\right)$

$$
C_{1}\left(0, s_{2}, 0\right) \equiv C_{1}\left(0, s_{2}\right)=-C_{0}\left(s_{2}\right) .
$$

Limit $\mathrm{V}\left(s_{2}=s_{3}=0\right)$

$$
\begin{aligned}
C_{1}\left(s_{1}, 0,0\right) \equiv C_{1}\left(s_{1}, 0\right) & =\frac{B_{0}\left(s_{1}\right)-B_{0}(0)}{s_{1}} \\
& =\frac{4-D}{2(D-3)} C_{0}\left(s_{1}\right) .
\end{aligned}
$$

Limit VI $\left(s_{1}=s_{2}=0\right)$

$$
\begin{aligned}
C_{1}\left(0,0, s_{3}\right) \equiv C_{1}\left(s_{3}, 0\right) & =\frac{B_{0}\left(s_{3}\right)-B_{0}(0)}{s_{3}} \\
& =\frac{4-D}{2(D-3)} C_{0}\left(s_{3}\right) .
\end{aligned}
$$

One can see that Limits V and VI are specified by the same function $C_{1}(s, 0)$.

In the following it is convenient to use the notations:

$$
\begin{aligned}
& F\left(s_{1}, s_{2}\right)=\frac{B_{0}\left(s_{1}\right)-B_{0}\left(s_{2}\right)}{s_{1}-s_{2}}=\frac{4-D}{2(D-3)} C_{0}\left(s_{1}, s_{2}\right), \\
& G\left(s_{1}, s_{2}\right)=\frac{B_{0}\left(s_{1}\right)+B_{0}\left(s_{2}\right)}{s_{1}-s_{2}} \\
& H\left(s_{1}, s_{2}\right)=G\left(s_{1}, s_{2}\right)+\frac{s_{1}+s_{2}}{s_{1}-s_{2}} F\left(s_{1}, s_{2}\right) .
\end{aligned}
$$


Functions $F\left(s_{1}, s_{2}\right)$ and $G\left(s_{1}, s_{2}\right)$ are manifestly symmetric resp. antisymmetric under exchange of variables $s_{1} \leftrightarrow s_{2}$. Function $F\left(s_{1}, s_{2}\right)$ obeys the useful identity:

$$
F\left(s_{1}, s_{2}\right)=\frac{s_{1} F\left(s_{1}, 0\right)-s_{2} F\left(s_{2}, 0\right)}{s_{1}-s_{2}} .
$$

Function $H\left(s_{1}, s_{2}\right)$ has the following property::

$$
H(s, 0)=-H(0, s)=\frac{2 B_{0}(s)}{s} .
$$

Furthermore we use the following relations:

$$
\begin{aligned}
& C_{0}(s, 0)=C_{0}(0, s)=-C_{1}(0, s)=2 F(s, 0) \frac{D-3}{4-D}, \\
& C_{1}(s, 0)=F(s, 0) .
\end{aligned}
$$

Also we introduce the functions $\alpha_{ \pm}\left(s_{1}, s_{2}, s_{3}\right), \beta_{ \pm}\left(s_{1}, s_{2}, s_{3}\right), \gamma_{ \pm}\left(s_{1}, s_{2}, s_{3}\right)$, and $\sigma_{ \pm}\left(s_{1}, s_{2}, s_{3}\right)$ depending on three different Mandelstam variables with $s_{1} \neq s_{2} \neq s_{3}$ and $s_{1}+s_{2}+s_{3}=Q^{2}$ :

$$
\begin{aligned}
& \alpha_{ \pm}\left(s_{1}, s_{2}, s_{3}\right)=\frac{\left(Q^{2}-s_{1}\right)^{2}-\left(Q^{2} s_{2}-s_{1} s_{3}\right)}{s\left(Q^{2} \pm s_{1}\right)}, \\
& \beta_{ \pm}\left(s_{1}, s_{2}, s_{3}\right)=\frac{\left(Q^{2}-s_{1}\right)^{2}+\left(Q^{2} s_{2}-s_{1} s_{3}\right)}{s\left(Q^{2} \pm s_{1}\right)} \\
& \gamma_{ \pm}\left(s_{1}, s_{2}, s_{3}\right)=\frac{\left(Q^{2}-s_{1}\right)^{2}+s_{1}\left(s_{2}-s_{3}\right)}{s\left(Q^{2} \pm s_{1}\right)} \\
& \sigma_{ \pm}\left(s_{1}, s_{2}, s_{3}\right)=\frac{\left(Q^{2}-s_{1}\right)^{4}+s_{1}\left(s_{2}-s_{3}\right)\left(Q^{2} s_{3}-s_{1} s_{2}\right)}{s^{2}\left(Q^{2} \pm s_{1}\right)^{2}} .
\end{aligned}
$$

It is important to stress that the numerator of functions $\alpha_{ \pm}\left(s_{1}, s_{2}, s_{3}\right)$ is manifestly symmetric under exchange of variables $s_{1} \leftrightarrow s_{2}$ and obeys the identities

$$
\begin{aligned}
& \left(Q^{2}-s_{1}\right)\left(Q^{2}-s_{2}\right)-2 s_{1} s_{2} \\
& =Q^{2} s_{3}-s_{1} s_{2} \\
& =\left(Q^{2}-s_{1}\right)^{2}-Q^{2} s_{2}+s_{1} s_{3} \\
& =\left(Q^{2}-s_{2}\right)^{2}-Q^{2} s_{1}+s_{2} s_{3} .
\end{aligned}
$$

Another interesting identity reads:

$$
\gamma_{ \pm}\left(s_{1}, s_{2}, s_{3}\right) \gamma_{ \pm}\left(s_{1}, s_{3}, s_{2}\right)=\frac{\left(s_{2}+s_{3}\right)^{4}-s_{1}^{2}\left(s_{2}-s_{3}\right)^{2}}{s^{2}\left(Q^{2} \pm s_{1}\right)^{2}} .
$$

In above formulas the set of variables $\left(s_{1}, s_{2}, s_{3}\right)$ is not fixed to a specific ordering of $(s, t, u)$ variables, i.e. all possible 6 sets of arguments due to permutations of $s_{1}, s_{2}$, and $s_{3}$ could occur.

Now we are in the position to list the scalar functions, which occur in the expansion of vectors triangle integrals. In doing this we group the scalar functions into pairs taking into account symmetric relations between them. Most of the relations between the scalar 
functions are derived using the change of the external momenta: $p_{1} \rightarrow-p_{2}, p_{2} \rightarrow-p_{1}$, $k_{1} \rightarrow-k_{1}, q \rightarrow-q$ leading to the following change of Mandelstam variables and the momenta from orthogonal basis: $s \rightarrow s, u \rightarrow t, t \rightarrow u, P \rightarrow-P, R \rightarrow R, T \rightarrow-T$. It concerns the pairs of the integrals $\left(C_{1 J}^{015}, C_{1 J}^{026}\right),\left(C_{1 J}^{016}, C_{1 J}^{025}\right),\left(C_{1 J}^{018}, C_{1 J}^{024}\right),\left(C_{1 J}^{019}, C_{1 J}^{023}\right)$, $\left(C_{1 J}^{035}, C_{1 J}^{069}\right),\left(C_{1 J}^{046}, C_{1 J}^{058}\right)$. The relations between the integrals $C_{1 J}^{038}$ and $C_{1 K}^{069}$ are obtained by inverse of the loop momentum $k \rightarrow-k$. The relations between the integrals $C_{1 J}^{012}$ and $C_{1 J}^{017}$ are obtained by change of the loop momentum $k \rightarrow-\left(k+p_{1}\right)$, while in the case of $C_{1 J}^{078}$ and $C_{1 J}^{079}$ integrals we apply the following change of the loop momentum $k \rightarrow-(k+P)$.

We proceed using the following strategy:

1. indicate the pairs of loop integrals obeying specific symmetry relations;

2. display the sets of corresponding scalar functions and relations between them;

3. write down explicit results for the scalar functions.

Loop integrals $C^{\mu ; 012}$ and $C^{\mu ; 017}$. Sets of the scalar functions:

$$
\begin{aligned}
& C_{1 P}^{012}(s), C_{1 R}^{017}(s), C_{1 T}^{012}(s), \\
& C_{1 P}^{017}(s), C_{1 R}^{017}(s), C_{1 T}^{017}(s) .
\end{aligned}
$$

Relations:

$$
\left\{\begin{array}{l}
C_{1 P}^{012}(s)=-C_{1 P}^{017}(s)-\frac{1}{2} C_{0}(s, 0), \\
C_{1 R}^{012}(s)=-C_{1 R}^{017}(s)-\frac{1}{2} C_{0}(s, 0), \\
C_{1 T}^{012}(s)=-C_{1 T}^{017}(s)
\end{array}\right.
$$

Explicit results:

$$
C_{1 P}^{012}(s)=C_{1 T}^{012}(s)=0, \quad C_{1 R}^{012}(s)=C_{1}(s, 0)=F(s, 0) .
$$

Loop integrals $C^{\mu ; 015}$ and $C^{\mu ; 026}$. Sets of the scalar functions:

$$
\begin{aligned}
& C_{1 P}^{015}\left(s, u, Q^{2}\right), C_{1 R}^{015}\left(s, u, Q^{2}\right), C_{1 T}^{015}(u), \\
& C_{1 P}^{026}\left(s, t, Q^{2}\right), C_{1 R}^{026}\left(s, t, Q^{2}\right), C_{1 T}^{026}(t) .
\end{aligned}
$$

Relations:

$$
\left\{\begin{array}{l}
C_{1 P}^{015}\left(s, u, Q^{2}\right)=-C_{1 P}^{026}\left(s, u, Q^{2}\right) \\
C_{1 R}^{015}\left(s, u, Q^{2}\right)=+C_{1 R}^{026}\left(s, u, Q^{2}\right) \\
C_{1 T}^{015}(u)=-C_{1 T}^{026}(u)
\end{array}\right.
$$

Explicit results:

$$
\begin{aligned}
C_{1 P}^{015}\left(s, u, Q^{2}\right) & =\frac{C_{1}(0, u)}{2}+\frac{C_{1}(u, 0)}{2} \frac{Q^{2}-2 s}{s}=F(u, 0)\left[\frac{1}{D-4}+\frac{Q^{2}}{2 s}\right], \\
C_{1 R}^{015}\left(s, u, Q^{2}\right) & =\frac{C_{1}(0, u)}{2}-\frac{C_{1}(u, 0)}{2} \frac{Q^{2}-2 t}{s}=C_{1 P}^{015}\left(s, u, Q^{2}\right)-F(u, 0) \frac{u}{s}, \\
C_{1 T}^{015}(u) & =-C_{1}(u, 0)=-F(u, 0) .
\end{aligned}
$$


Loop integrals $C^{\mu ; 016}$ and $C^{\mu ; 025}$. Sets of the scalar functions:

$$
\begin{aligned}
& C_{1 P}^{016}\left(s, t, Q^{2}\right), C_{1 R}^{016}\left(s, t, Q^{2}\right), C_{1 T}^{016}\left(t, Q^{2}\right), \\
& C_{1 P}^{025}\left(s, u, Q^{2}\right), C_{1 R}^{025}\left(s, u, Q^{2}\right), C_{1 T}^{025}\left(u, Q^{2}\right) .
\end{aligned}
$$

Relations:

$$
\left\{\begin{array}{l}
C_{1 P}^{016}\left(s, t, Q^{2}\right)=-C_{1 P}^{025}\left(s, t, Q^{2}\right), \\
C_{1 R}^{016}\left(s, t, Q^{2}\right)=+C_{1 R}^{025}\left(s, t, Q^{2}\right), \\
C_{1 T}^{016}\left(t, Q^{2}\right)=-C_{1 T}^{025}\left(t, Q^{2}\right) .
\end{array}\right.
$$

Explicit results:

$$
\begin{aligned}
C_{1 P}^{016}\left(s, t, Q^{2}\right) & =\frac{C_{1}\left(0, t, Q^{2}\right)}{2}-\frac{C_{1}\left(t, 0, Q^{2}\right)}{2} \gamma_{-}(t, u, s) \\
& =-F\left(Q^{2}, t\right)\left[\frac{t}{\left(Q^{2}-t\right)(D-4)}+\frac{Q^{2}}{2 s}\right]+F\left(Q^{2}, 0\right) \frac{Q^{2}}{2\left(Q^{2}-t\right)} \\
C_{1 R}^{016}\left(s, t, Q^{2}\right) & =\frac{C_{1}\left(0, t, Q^{2}\right)}{2}+\frac{C_{1}\left(t, 0, Q^{2}\right)}{2} \gamma_{-}(t, s, u) \\
& =C_{1 P}^{016}\left(s, t, Q^{2}\right)+F\left(Q^{2}, t\right) \frac{Q^{2}-t}{s} \\
C_{1 T}^{016}\left(t, Q^{2}\right) & =C_{1}\left(t, 0, Q^{2}\right)=F\left(Q^{2}, t\right) .
\end{aligned}
$$

Loop integrals $C^{\mu ; 018}$ and $C^{\mu ; 024}$. Sets of the scalar functions:

$$
\begin{aligned}
& C_{1 P}^{018}\left(s, t, Q^{2}\right), C_{1 R}^{018}\left(s, t, Q^{2}\right), C_{1 T}^{018}\left(t, Q^{2}\right) \\
& C_{1 P}^{024}\left(s, u, Q^{2}\right), C_{1 R}^{024}\left(s, u, Q^{2}\right), C_{1 T}^{024}\left(u, Q^{2}\right) .
\end{aligned}
$$

Relations:

$$
\left\{\begin{array}{l}
C_{1 P}^{018}\left(s, t, Q^{2}\right)=-C_{1 P}^{024}\left(s, t, Q^{2}\right), \\
C_{1 R}^{018}\left(s, t, Q^{2}\right)=+C_{1 R}^{024}\left(s, t, Q^{2}\right), \\
C_{1 T}^{018}\left(t, Q^{2}\right)=-C_{1 T}^{024}\left(t, Q^{2}\right) .
\end{array}\right.
$$

Explicit results:

$$
\begin{aligned}
C_{1 P}^{018}\left(s, t, Q^{2}\right) & =\frac{C_{1}\left(0, Q^{2}, t\right)}{2}+\frac{C_{1}\left(Q^{2}, 0, t\right)}{2} \alpha_{-}(t, s, u) \\
& =F\left(Q^{2}, t\right)\left[\frac{Q^{2}}{\left(Q^{2}-t\right)(D-4)}+\frac{Q^{2}+2 s}{2 s}\right]-F\left(Q^{2}, 0\right) \frac{Q^{2}}{2\left(Q^{2}-t\right)} \\
C_{1 R}^{018}\left(s, t, Q^{2}\right) & =\frac{C_{1}\left(0, Q^{2}, t\right)}{2}-\frac{C_{1}\left(Q^{2}, 0, t\right)}{2} \beta_{-}(t, s, u) \\
& =C_{1 P}^{018}\left(s, t, Q^{2}\right)-F\left(Q^{2}, t\right) \frac{Q^{2}-t}{s} \\
C_{1 T}^{018}\left(t, Q^{2}\right) & =-C_{1}\left(Q^{2}, 0, t\right)=-F\left(Q^{2}, t\right)
\end{aligned}
$$


Loop integrals $C^{\mu ; 019}$ and $C^{\mu ; 023}$. Sets of the scalar functions:

$$
\begin{aligned}
& C_{1 P}^{019}\left(s, u, Q^{2}\right), C_{1 R}^{019}\left(s, u, Q^{2}\right), C_{1 T}^{019}(u), \\
& C_{1 P}^{023}\left(s, t, Q^{2}\right), C_{1 R}^{023}\left(s, t, Q^{2}\right), C_{1 T}^{023}(t) .
\end{aligned}
$$

Relations:

$$
\left\{\begin{array}{l}
C_{1 P}^{019}\left(s, u, Q^{2}\right)=-C_{1 P}^{023}\left(s, u, Q^{2}\right) \\
C_{1 R}^{019}\left(s, u, Q^{2}\right)=+C_{1 R}^{023}\left(s, u, Q^{2}\right) \\
C_{1 T}^{019}(u)=-C_{1 T}^{023}(u)
\end{array}\right.
$$

Explicit results:

$$
\begin{aligned}
C_{1 P}^{019}\left(s, u, Q^{2}\right) & =-\frac{C_{1}(u, 0)}{2 s}\left(Q^{2}-2 s\right)=-F(u, 0) \frac{Q^{2}-2 s}{2 s}, \\
C_{1 R}^{019}\left(s, u, Q^{2}\right) & =-\frac{C_{1}(u, 0)}{2 s}\left(Q^{2}-2 t\right)=-F(u, 0) \frac{Q^{2}-2 t}{2 s}, \\
C_{1 T}^{019}(u) & =C_{1}(u, 0)=F(u, 0) .
\end{aligned}
$$

Loop integrals $C^{\mu ; 035}$ and $C^{\mu ; 069}$. Sets of the scalar functions:

$$
\begin{aligned}
& C_{1 P}^{035}\left(s, u, Q^{2}\right), C_{1 R}^{035}\left(s, u, Q^{2}\right), C_{1 T}^{035}(u), \\
& C_{1 P}^{069}\left(s, t, Q^{2}\right), C_{1 R}^{069}\left(s, t, Q^{2}\right), C_{1 T}^{069}(t) .
\end{aligned}
$$

Relations:

$$
\left\{\begin{array}{l}
C_{1 P}^{035}\left(s, u, Q^{2}\right)=-C_{1 P}^{069}\left(s, u, Q^{2}\right) \\
C_{1 R}^{035}\left(s, u, Q^{2}\right)=+C_{1 R}^{069}\left(s, u, Q^{2}\right) \\
C_{1 T}^{035}(u)=-C_{1 T}^{069}(u)
\end{array}\right.
$$

Explicit results:

$$
\begin{aligned}
C_{1 P}^{035}\left(s, u, Q^{2}\right) & =\frac{C_{1}(0, u)}{2} \frac{Q^{2}-s}{s}-\frac{C_{1}(u, 0)}{2} \frac{Q^{2}-2 s}{s}=F(u, 0)\left[\frac{Q^{2}-s}{s(D-4)}+\frac{Q^{2}}{2 s}\right] \\
C_{1 R}^{035}\left(s, u, Q^{2}\right) & =\frac{C_{1}(0, u)}{2} \frac{t-u}{s}+\frac{C_{1}(u, 0)}{2} \frac{Q^{2}-2 t}{s}=C_{1 P}^{035}\left(s, u, Q^{2}\right)-\frac{F(u, 0) u}{s} \frac{D-2}{D-4}, \\
C_{1 T}^{035}(u) & =-C_{1}(0, u)+C_{1}(u, 0)=-F(u, 0) \frac{D-2}{D-4} .
\end{aligned}
$$

Loop integrals $C^{\mu ; 038}$ and $C^{\mu ; 049}$. Sets of the scalar functions:

$$
\begin{aligned}
& C_{1 P}^{038}\left(s, Q^{2}\right), C_{1 R}^{038}\left(s, u, Q^{2}\right), C_{1 T}^{038}\left(s, Q^{2}\right), \\
& C_{1 P}^{049}\left(s, Q^{2}\right), C_{1 R}^{049}\left(s, u, Q^{2}\right), C_{1 T}^{049}\left(s, Q^{2}\right) .
\end{aligned}
$$

Relations:

$$
\left\{\begin{array}{l}
C_{1 P}^{038}\left(s, Q^{2}\right)=-C_{1 P}^{049}\left(s, Q^{2}\right) \\
C_{1 R}^{038}\left(s, u, Q^{2}\right)=-C_{1 R}^{049}\left(s, u, Q^{2}\right) \\
C_{1 T}^{038}\left(s, Q^{2}\right)=-C_{1 T}^{049}\left(s, Q^{2}\right)
\end{array}\right.
$$


Explicit results:

$$
\begin{aligned}
C_{1 P}^{038}\left(s, Q^{2}\right) & =\frac{Q^{2}-s}{2 s}\left[C_{1}\left(0, Q^{2}, s\right)-C_{1}\left(Q^{2}, 0, s\right)\right] \\
& =\frac{Q^{2}}{s}\left[F\left(Q^{2}, s\right) \frac{D-3}{D-4}-\frac{F\left(Q^{2}, 0\right)}{2}\right], \\
C_{1 R}^{038}\left(s, u, Q^{2}\right) & =\frac{u-t}{2 s} C_{1 T}^{038}\left(s, Q^{2}\right), \\
C_{1 T}^{038}\left(s, Q^{2}\right) & =-\left[C_{1}\left(0, Q^{2}, s\right)-C_{1}\left(Q^{2}, 0, s\right) \frac{Q^{2}+s}{Q^{2}-s}\right] \\
& =-\frac{2 s}{Q^{2}-s}\left[C_{1 P}^{038}\left(s, Q^{2}\right)-F\left(Q^{2}, s\right)\right] .
\end{aligned}
$$

Loop integrals $C^{\mu ; 046}$ and $C^{\mu ; 058}$. Sets of the scalar functions:

$$
\begin{aligned}
& C_{1 P}^{046}\left(s, t, Q^{2}\right), C_{1 R}^{046}\left(s, t, Q^{2}\right), C_{1 T}^{046}\left(t, Q^{2}\right), \\
& C_{1 P}^{058}\left(s, u, Q^{2}\right), C_{1 R}^{058}\left(s, u, Q^{2}\right), C_{1 T}^{058}\left(u, Q^{2}\right) .
\end{aligned}
$$

Relations:

$$
\left\{\begin{array}{l}
C_{1 P}^{046}\left(s, t, Q^{2}\right)=-C_{1 P}^{058}\left(s, t, Q^{2}\right) \\
C_{1 R}^{046}\left(s, t, Q^{2}\right)=+C_{1 R}^{058}\left(s, t, Q^{2}\right) \\
C_{1 T}^{046}\left(t, Q^{2}\right)=-C_{1 T}^{058}\left(t, Q^{2}\right)
\end{array}\right.
$$

Explicit results:

$$
\begin{aligned}
C_{1 P}^{046}\left(s, t, Q^{2}\right) & =\frac{C_{1}\left(Q^{2}, t, 0\right)}{2} \alpha_{+}(t, s, u)-\frac{C_{1}\left(t, Q^{2}, 0\right)}{2} \gamma_{+}(t, u, s) \\
& =-F\left(Q^{2}, t\right)\left[\frac{\left(Q^{2}-t\right)^{2}+u t}{\left(Q^{2}-t\right) s(D-4)}+\frac{Q^{2}}{2 s}\right]-F\left(Q^{2}, 0\right) \frac{Q^{2}}{2\left(Q^{2}-t\right)}, \\
C_{1 R}^{046}\left(s, t, Q^{2}\right) & =-\frac{C_{1}\left(Q^{2}, t, 0\right)}{2} \beta_{+}(t, s, u)+\frac{C_{1}\left(t, Q^{2}, 0\right)}{2} \gamma_{+}(t, s, u) \\
& =C_{1 P}^{046}\left(s, t, Q^{2}\right)+F\left(Q^{2}, t\right) \frac{Q^{2}-t}{s} \frac{D-2}{D-4} \\
C_{1 T}^{046}\left(t, Q^{2}\right) & =-\frac{Q^{2}-t}{Q^{2}+t}\left[C_{1}\left(Q^{2}, t, 0\right)-C_{1}\left(t, Q^{2}, 0\right)\right] \\
& =F\left(Q^{2}, t\right) \frac{D-2}{D-4} .
\end{aligned}
$$

Loop integrals $C^{\mu ; 078}$ and $C^{\mu ; 079}$. Sets of the scalar functions:

$$
\begin{aligned}
& C_{1 P}^{078}\left(s, Q^{2}\right), C_{1 R}^{046}\left(s, u, Q^{2}\right), C_{1 T}^{046}\left(s, Q^{2}\right), \\
& C_{1 P}^{079}\left(s, Q^{2}\right), C_{1 R}^{069}\left(s, t, Q^{2}\right), C_{1 T}^{058}\left(s, Q^{2}\right) .
\end{aligned}
$$

Relations:

$$
\left\{\begin{array}{l}
C_{1 P}^{078}\left(s, Q^{2}\right)=-C_{1 P}^{079}\left(s, Q^{2}\right)-C_{0}\left(Q^{2}, s\right) \\
C_{1 R}^{078}\left(s, u, Q^{2}\right)=-C_{1 R}^{079}\left(s, u, Q^{2}\right) \\
C_{1 T}^{078}\left(s, Q^{2}\right)=-C_{1 T}^{079}\left(s, Q^{2}\right)
\end{array}\right.
$$


Explicit results:

$$
\begin{aligned}
C_{1 P}^{078}\left(s, Q^{2}\right) & =\frac{C_{1}\left(Q^{2}, s, 0\right)}{2} \frac{\left(Q^{2}-s\right)^{2}}{s\left(Q^{2}+s\right)}=F\left(Q^{2}, s\right) \frac{D-3}{D-4}+F\left(Q^{2}, 0\right) \frac{Q^{2}}{2 s}, \\
C_{1 R}^{078}\left(s, u, Q^{2}\right) & =\frac{u-t}{2 s} C_{1 T}^{078}\left(s, Q^{2}\right), \\
C_{1 T}^{078}\left(s, Q^{2}\right) & =C_{1}\left(s, Q^{2}, 0\right) \frac{2 s}{Q^{2}+s}-C_{1}\left(Q^{2}, s, 0\right) \\
& =\frac{2 s}{Q^{2}-s}\left[\frac{F\left(Q^{2}, s\right)}{D-4}-F\left(Q^{2}, 0\right) \frac{Q^{2}}{2 s}\right] .
\end{aligned}
$$

\section{A.2 Tensor triangle integrals}

In this section we show results for the triangle tensor rank-2 integrals:

$$
\begin{aligned}
C_{2}^{\mu \nu ; 0 i j}= & \left(i \pi^{2}\right)^{-1} \mu^{4-D} \int d^{D} k \frac{k^{\mu}}{\Delta_{0} \Delta_{i} \Delta_{j}}=g_{\perp}^{\mu \nu ; 3} C_{2 g}^{0 i j}+P^{\mu} P^{\nu} C_{2 P P}^{0 i j}+R^{\mu} R^{\nu} C_{2 R R}^{0 i j} \\
& +T^{\mu} T^{\nu} C_{2 T T}^{0 i j}+\{P R\}^{\mu \nu} C_{2 P R}^{0 i j}+\{P T\}^{\mu \nu} C_{2 P T}^{0 i j}+\{R T\}^{\mu \nu} C_{2 R T}^{0 i j},
\end{aligned}
$$

where $\{A B\}^{\mu \nu}=A^{\mu} B^{\nu}+A^{\nu} B^{\mu}$.

All scalar functions are expressed in terms of three scalar functions derived in eq. (2.30):

$$
\begin{aligned}
C_{21}\left(s_{1}, s_{2}, s_{3}\right)= & C_{2 ; g}\left(s_{1}, s_{2}, s_{3}\right) \\
= & \frac{1}{D-2}\left[B_{0}\left(s_{3}\right)+\lambda \frac{s_{1} C_{22}\left(s_{1}, s_{2}, s_{3}\right)+s_{2} C_{22}\left(s_{2}, s_{1}, s_{3}\right)}{\left(s_{1}+s_{2}-s_{3}\right)^{2}}\right. \\
& \left.-\frac{\lambda C_{23}\left(s_{1}, s_{2}, s_{3}\right)}{s_{1}+s_{2}-s_{3}}\right], \\
C_{22}\left(s_{1}, s_{2}, s_{3}\right)= & C_{2 ; l_{1} l_{1}}\left(s_{1}, s_{2}, s_{3}\right)=C_{2 ; l_{2} l_{2}}\left(s_{2}, s_{1}, s_{3}\right) \\
= & \frac{\left(s_{1}+s_{2}-s_{3}\right)^{2}}{2 \lambda^{2}}\left[-\left(s_{1}+s_{2}-s_{3}\right) B_{0}\left(s_{1}\right)+\left(s_{1}+3 s_{2}-s_{3}\right) B_{0}\left(s_{3}\right)\right. \\
& \left.-\frac{2 s_{2} \lambda}{s_{1}+s_{2}-s_{3}} C_{1}\left(s_{1}, s_{2}, s_{3}\right)\right], \\
C_{23}\left(s_{1}, s_{2}, s_{3}\right)= & C_{2 ; l_{1} l_{2}\left(s_{1}, s_{2}, s_{3}\right)} \\
= & \frac{\left(s_{1}+s_{2}-s_{3}\right)^{2}}{2 \lambda^{2}}\left[-s_{1} B_{0}\left(s_{1}\right)-s_{2} B_{0}\left(s_{2}\right)+\left(2 s_{1}+2 s_{2}-s_{3}\right) B_{0}\left(s_{3}\right)\right. \\
& \left.-\frac{\lambda}{s_{1}+s_{2}-s_{3}}\left(s_{1} C_{1}\left(s_{1}, s_{2}, s_{3}\right)+s_{2} C_{1}\left(s_{2}, s_{1}, s_{3}\right)\right)\right] .
\end{aligned}
$$

Now we display triangle tensor functions.

Loop integrals $C^{\mu \nu ; 012}$ and $C^{\mu \nu ; 017}$. Sets of the scalar functions:

$$
\begin{aligned}
& C_{2 g}^{012}(s), C_{2 P P}^{012}(s), C_{2 R R}^{012}(s), C_{2 T T}^{012}(s), C_{2 P R}^{012}(s), C_{2 P T}^{012}(s), C_{2 R T}^{012}(s), \\
& C_{2 g}^{017}(s), C_{2 P P}^{017}(s), C_{2 R R}^{017}(s), C_{2 T T}^{017}(s), C_{2 P R}^{017}(s), C_{2 P T}^{017}(s), C_{2 R T}^{017}(s) .
\end{aligned}
$$


Relations:

$$
\left\{\begin{array}{l}
C_{2 g}^{012}(s)=+C_{2 g}^{017}(s) \\
C_{2 P P}^{012}(s)=+C_{2 P P}^{017}(s)-\frac{1}{4} C_{0}(s, 0) \\
C_{2 R R}^{012}(s)=+C_{2 R R}^{017}(s)-F(s, 0)-\frac{1}{4} C_{0}(s, 0) \\
C_{2 T T}^{012}(s)=+C_{2 T T}^{017}(s) \\
C_{2 P R}^{012}(s)=+C_{2 P R}^{017}(s)-\frac{1}{2} F(s, 0)-\frac{1}{4} C_{0}(s, 0), \\
C_{2 P T}^{012}(s)=+C_{2 P T}^{017}(s) \\
C_{2 R T}^{012}(s)=+C_{2 R T}^{017}(s) .
\end{array}\right.
$$

Explicit results:

$$
\begin{aligned}
& C_{2 g}^{012}(s)=C_{21}(0,0, s)=\frac{F(s, 0)+G(s, 0)}{D-2} \frac{s}{4} \\
& C_{2 P P}^{012}(s)=\frac{C_{22}(0,0, s)-C_{23}(0,0, s)}{2}=\frac{G(s, 0)-F(s, 0)}{8} \\
& C_{2 R R}^{012}(s)=\frac{C_{22}(0,0, s)+C_{23}(0,0, s)}{2}=-\frac{G(s, 0)+3 F(s, 0)}{8} \\
& C_{2 T T}^{012}(s)=-\frac{s}{u t} C_{2 g}^{012}(s) \\
& C_{2 P R}^{012}(s)=C_{2 P T}^{012}(s)=C_{2 R T}^{012}(s)=0
\end{aligned}
$$

Loop integrals $C^{\mu \nu ; 015}$ and $C^{\mu \nu ; 026}$. Sets of the scalar functions:

$$
\begin{aligned}
& C_{2 g}^{015}\left(s, u, Q^{2}\right), C_{2 P P}^{015}\left(s, u, Q^{2}\right), C_{2 R R}^{015}\left(s, u, Q^{2}\right), C_{2 T T}^{015}\left(s, u, Q^{2}\right), \\
& C_{2 P R}^{015}\left(s, u, Q^{2}\right), C_{2 P T}^{015}\left(s, u, Q^{2}\right), C_{2 R T}^{015}\left(s, u, Q^{2}\right) \\
& C_{2 g}^{026}\left(s, t, Q^{2}\right), C_{2 P P}^{026}\left(s, t, Q^{2}\right), C_{2 R R}^{026}\left(s, t, Q^{2}\right), C_{2 T T}^{026}\left(s, t, Q^{2}\right) \\
& C_{2 P R}^{026}\left(s, t, Q^{2}\right), C_{2 P T}^{026}\left(s, t, Q^{2}\right), C_{2 R T}^{026}\left(s, t, Q^{2}\right) .
\end{aligned}
$$

Relations:

$$
\left\{\begin{array}{l}
C_{2 g}^{015}\left(s, u, Q^{2}\right)=+C_{2 g}^{026}\left(s, u, Q^{2}\right), \\
C_{2 P P}^{015}\left(s, u, Q^{2}\right)=+C_{2 P P}^{026}\left(s, u, Q^{2}\right), \\
C_{2 R R}^{015}\left(s, u, Q^{2}\right)=+C_{2 R R}^{026}\left(s, u, Q^{2}\right), \\
C_{2 T T}^{015}\left(s, u, Q^{2}\right)=+C_{2 T T}^{026}\left(s, u, Q^{2}\right), \\
C_{2 P R}^{015}\left(s, u, Q^{2}\right)=-C_{2 P R}^{026}\left(s, u, Q^{2}\right), \\
C_{2 P T}^{015}\left(s, u, Q^{2}\right)=+C_{2 P T}^{026}\left(s, u, Q^{2}\right), \\
C_{2 R T}^{015}\left(s, u, Q^{2}\right)=-C_{2 R T}^{026}\left(s, u, Q^{2}\right) .
\end{array}\right.
$$

Explicit results:

$$
\begin{aligned}
C_{2 g}^{015}\left(s, u, Q^{2}\right)= & C_{21}(0, u, 0)=\frac{F(u, 0)+G(u, 0)}{D-2} \frac{u}{4} \\
C_{2 P P}^{015}\left(s, u, Q^{2}\right)= & -\frac{t}{s u} C_{21}(0, u, 0)+\frac{C_{22}(0, u, 0)}{4}+\frac{\left(Q^{2}-2 s\right)^{2}}{4 s^{2}} C_{22}(u, 0,0) \\
& +\frac{Q^{2}-2 s}{2 s} C_{23}(0, u, 0)=-\frac{F(u, 0)}{2}\left[\frac{1}{D-4}+\frac{Q^{4}}{4 s^{2}}\right]-\frac{F(u, 0)+G(u, 0)}{D-2} \frac{t}{4 s} \\
& +[G(u, 0)-F(u, 0)] \frac{Q^{2}-s}{8 s}
\end{aligned}
$$




$$
\begin{aligned}
& C_{2 R R}^{015}\left(s, u, Q^{2}\right)=-\frac{t}{s u} C_{21}(0, u, 0)+\frac{C_{22}(0, u, 0)}{4}+\frac{\left(Q^{2}-2 t\right)^{2}}{4 s^{2}} C_{22}(u, 0,0) \\
& -\frac{Q^{2}-2 t}{2 s} C_{23}(0, u, 0) \\
& =C_{2 P P}^{015}\left(s, u, Q^{2}\right)+\frac{u}{4 s}\left[2 F(u, 0) \frac{Q^{2}-u}{s}+F(u, 0)-G(u, 0)\right], \\
& C_{2 T T}^{015}\left(s, u, Q^{2}\right)=-\frac{s}{t u} C_{21}(0, u, 0)+C_{22}(u, 0,0)=-\frac{F(u, 0)+G(u, 0)}{D-2} \frac{s}{4 t}-\frac{F(u, 0)}{2}, \\
& C_{2 P R}^{015}\left(s, u, Q^{2}\right)=-\frac{t}{s u} C_{21}(0, u, 0)+\frac{C_{22}(0, u, 0)}{4}-\frac{\left(Q^{2}-2 s\right)\left(Q^{2}-2 t\right)}{4 s^{2}} C_{22}(u, 0,0) \\
& +\frac{t-s}{2 s} C_{23}(0, u, 0)=C_{2 P P}^{015}\left(s, u, Q^{2}\right) \\
& +\frac{u}{8 s}\left[2 F(u, 0) \frac{Q^{2}}{s}+F(u, 0)-G(u, 0)\right] \\
& C_{2 P T}^{015}\left(s, u, Q^{2}\right)=\frac{C_{21}(0, u, 0)}{u}-\frac{Q^{2}-2 s}{2 s} C_{22}(u, 0,0)-\frac{C_{23}(0, u, 0)}{2} \\
& =\frac{F(u, 0)+G(u, 0)}{4(D-2)}+F(u, 0) \frac{Q^{2}}{4 s}+\frac{F(u, 0)-G(u, 0)}{8}, \\
& C_{2 R T}^{015}\left(s, u, Q^{2}\right)=\frac{C_{21}(0, u, 0)}{u}+\frac{Q^{2}-2 t}{2 s} C_{22}(u, 0,0)-\frac{C_{23}(0, u, 0)}{2} \\
& =C_{2 P T}^{015}\left(s, u, Q^{2}\right)-F(u, 0) \frac{u}{2 s} .
\end{aligned}
$$

Loop integrals $C^{\mu \nu ; 016}$ and $C^{\mu \nu ; 025}$. Sets of the scalar functions:

$$
\begin{aligned}
& C_{2 g}^{016}\left(s, t, Q^{2}\right), C_{2 P P}^{016}\left(s, t, Q^{2}\right), C_{2 R R}^{016}\left(s, t, Q^{2}\right), C_{2 T T}^{016}\left(s, t, Q^{2}\right) \\
& C_{2 P R}^{016}\left(s, t, Q^{2}\right), C_{2 P T}^{016}\left(s, t, Q^{2}\right), C_{2 R T}^{016}\left(s, t, Q^{2}\right) \\
& C_{2 g}^{025}\left(s, u, Q^{2}\right), C_{2 P P}^{025}\left(s, u, Q^{2}\right), C_{2 R R}^{025}\left(s, u, Q^{2}\right), C_{2 T T}^{025}\left(s, u, Q^{2}\right), \\
& C_{2 P R}^{025}\left(s, u, Q^{2}\right), C_{2 P T}^{025}\left(s, u, Q^{2}\right), C_{2 R T}^{025}\left(s, u, Q^{2}\right) .
\end{aligned}
$$

Relations:

Explicit results:

$$
\left\{\begin{array}{l}
C_{2 g}^{016}\left(s, t, Q^{2}\right)=+C_{2 g}^{025}\left(s, t, Q^{2}\right) \\
C_{2 P P}^{016}\left(s, t, Q^{2}\right)=+C_{2 P P}^{025}\left(s, t, Q^{2}\right), \\
C_{2 R R}^{016}\left(s, t, Q^{2}\right)=+C_{2 R R}^{025}\left(s, t, Q^{2}\right) \\
C_{2 T T}^{016}\left(s, t, Q^{2}\right)=+C_{2 T T}^{025}\left(s, t, Q^{2}\right) \\
C_{2 P R}^{016}\left(s, t, Q^{2}\right)=-C_{2 P R}^{025}\left(s, t, Q^{2}\right) \\
C_{2 P T}^{016}\left(s, t, Q^{2}\right)=+C_{2 P T}^{025}\left(s, t, Q^{2}\right) \\
C_{2 R T}^{016}\left(s, t, Q^{2}\right)=-C_{2 R T}^{025}\left(s, t, Q^{2}\right) .
\end{array}\right.
$$

$$
\begin{aligned}
C_{2 g}^{016}\left(s, t, Q^{2}\right)= & C_{21}\left(0, t, Q^{2}\right)=\frac{H\left(Q^{2}, t\right)}{D-2} \frac{Q^{2}-t}{4} \\
C_{2 P P}^{016}\left(s, t, Q^{2}\right)= & -\frac{u t C_{21}\left(0, t, Q^{2}\right)}{s\left(Q^{2}-t\right)^{2}}+\frac{C_{22}\left(0, t, Q^{2}\right)}{4}+\frac{\gamma_{-}(t, u, s)^{2}}{4} C_{22}\left(t, 0, Q^{2}\right) \\
& -\frac{\gamma_{-}(t, u, s)}{2} C_{23}\left(0, t, Q^{2}\right)
\end{aligned}
$$




$$
\begin{aligned}
&=-\frac{F\left(Q^{2}, t\right)}{2}\left[\frac{t^{2}}{\left(Q^{2}-t\right)^{2}} \frac{1}{D-4}+\frac{Q^{4}}{4 s^{2}}\right]-\frac{H\left(Q^{2}, t\right)}{D-2} \frac{u t}{4 s\left(Q^{2}-t\right)} \\
&+\frac{Q^{2}}{8 s}\left[G\left(Q^{2}, 0\right) \frac{\left(Q^{2}-t\right)^{2}+u t}{\left(Q^{2}-t\right)^{2}}+F\left(Q^{2}, 0\right) \frac{\left(Q^{2}-s\right)\left(Q^{2}-t\right)+s t}{\left(Q^{2}-t\right)^{2}}\right], \\
& C_{2 R R}^{016}\left(s, t, Q^{2}\right)=-\frac{u t C_{21}\left(0, t, Q^{2}\right)}{s\left(Q^{2}-t\right)^{2}}+\frac{C_{22}\left(0, t, Q^{2}\right)}{4}+\frac{\gamma_{-}(t, s, u)^{2}}{4} C_{22}\left(t, 0, Q^{2}\right) \\
&+\frac{\gamma_{-}(t, s, u)}{2} C_{23}\left(0, t, Q^{2}\right) \\
&= C_{2 P P}^{016}\left(s, t, Q^{2}\right)-\left[F\left(Q^{2}, t\right)(s-2 t)+G\left(Q^{2}, t\right) s\right] \frac{Q^{2}-t}{4 s^{2}}, \\
& C_{2 T T}^{016}\left(s, t, Q^{2}\right)=-\frac{s}{u t} C_{21}\left(0, t, Q^{2}\right)+C_{22}\left(t, 0, Q^{2}\right)=-\frac{F\left(Q^{2}, t\right)}{2}-\frac{H\left(Q^{2}, t\right)}{D-2} \frac{s\left(Q^{2}-t\right)}{4 u t}, \\
& C_{2 P R}^{016}\left(s, t, Q^{2}\right)=-\frac{u t}{s\left(Q^{2}-t\right)^{2}} C_{21}\left(0, t, Q^{2}\right)+\frac{C_{22}\left(0, t, Q^{2}\right)}{4} \\
&-\frac{\gamma_{-}(t, s, u) \gamma_{-}(t, u, s)}{4} C_{22}\left(t, 0, Q^{2}\right)+\frac{t(s-u)}{2 s\left(Q^{2}-t\right)} C_{23}\left(0, t, Q^{2}\right) \\
&= C_{2 P P}^{016}\left(s, t, Q^{2}\right)+\left[F\left(Q^{2}, t\right)\left(2 Q^{2}-s\right)-G\left(Q^{2}, t\right) s\right] \frac{Q^{2}-t}{8 s^{2}}, \\
& C_{2 P T}^{016}\left(s, t, Q^{2}\right)= \frac{C_{21}\left(0, t, Q^{2}\right)}{Q^{2}-t}-\frac{\gamma_{-}(t, u, s)}{2} C_{22}\left(t, 0, Q^{2}\right)+\frac{C_{23}\left(0, t, Q^{2}\right)}{2} \\
& \frac{H\left(Q^{2}, t\right)}{4(D-2)}+F\left(Q^{2}, t\right)\left[\frac{Q^{2}}{4 s}-\frac{1}{8}\right]-\frac{G\left(Q^{2}, t\right)}{8}, \\
& C_{2 R T}^{016}\left(s, t, Q^{2}\right)= \frac{C_{21}\left(0, t, Q^{2}\right)}{Q^{2}-t}+\frac{\gamma_{-}(t, s, u)}{2} C_{22}\left(t, 0, Q^{2}\right)+\frac{C_{23}\left(0, t, Q^{2}\right)}{2} \\
&= C_{2 P T}^{016}\left(s, t, Q^{2}\right)-F\left(Q^{2}, t\right) \frac{Q^{2}-t}{2 s} . \\
&(\mathrm{A} .55) \\
&=
\end{aligned}
$$

Loop integrals $C^{\mu \nu ; 018}$ and $C^{\mu \nu ; 024}$. Sets of the scalar functions:

$$
\begin{aligned}
& C_{2 g}^{018}\left(s, t, Q^{2}\right), C_{2 P P}^{018}\left(s, t, Q^{2}\right), C_{2 R R}^{018}\left(s, t, Q^{2}\right), C_{2 T T}^{018}\left(s, t, Q^{2}\right) \\
& C_{2 P R}^{018}\left(s, t, Q^{2}\right), C_{2 P T}^{018}\left(s, t, Q^{2}\right), C_{2 R T}^{018}\left(s, t, Q^{2}\right) \\
& C_{2 g}^{024}\left(s, u, Q^{2}\right), C_{2 P P}^{024}\left(s, u, Q^{2}\right), C_{2 R R}^{024}\left(s, u, Q^{2}\right), C_{2 T T}^{024}\left(s, u, Q^{2}\right) \\
& C_{2 P R}^{024}\left(s, u, Q^{2}\right), C_{2 P T}^{024}\left(s, u, Q^{2}\right), C_{2 R T}^{024}\left(s, u, Q^{2}\right)
\end{aligned}
$$

Relations:

$$
\left\{\begin{array}{l}
C_{2 g}^{018}\left(s, t, Q^{2}\right)=+C_{2 g}^{024}\left(s, t, Q^{2}\right), \\
C_{2 P P}^{018}\left(s, t, Q^{2}\right)=+C_{2 P P}^{024}\left(s, t, Q^{2}\right), \\
C_{2 R R}^{018}\left(s, t, Q^{2}\right)=+C_{2 R R}^{024}\left(s, t, Q^{2}\right), \\
C_{2 T T}^{018}\left(s, t, Q^{2}\right)=+C_{2 T T}^{024}\left(s, t, Q^{2}\right), \\
C_{2 P R}^{018}\left(s, t, Q^{2}\right)=-C_{2 P R}^{024}\left(s, t, Q^{2}\right), \\
C_{2 P T}^{018}\left(s, t, Q^{2}\right)=+C_{2 P T}^{024}\left(s, t, Q^{2}\right), \\
C_{2 R T}^{018}\left(s, t, Q^{2}\right)=-C_{2 R T}^{024}\left(s, t, Q^{2}\right) .
\end{array}\right.
$$


Explicit results:

$$
\begin{aligned}
C_{2 g}^{018}\left(s, t, Q^{2}\right)= & C_{21}\left(0, Q^{2}, t\right)=\frac{H\left(Q^{2}, t\right)}{D-2} \frac{Q^{2}-t}{4}, \\
C_{2 P P}^{018}\left(s, t, Q^{2}\right)= & -\frac{u t}{s\left(Q^{2}-t\right)^{2}} C_{21}\left(0, Q^{2}, t\right)+\frac{C_{22}\left(0, Q^{2}, t\right)}{4}+\frac{\alpha_{-}^{2}(t, s, u)}{4} C_{22}\left(Q^{2}, 0, t\right) \\
& +\frac{\alpha_{-}(t, s, u)}{2} C_{23}\left(0, Q^{2}, t\right)=-\frac{F\left(Q^{2}, t\right)}{2}\left[\frac{Q^{4}}{\left(Q^{2}-t\right)^{2}(D-4)}+\frac{\left(Q^{2}+2 s\right)^{2}}{4 s^{2}}\right] \\
& -\frac{H\left(Q^{2}, t\right)}{D-2} \frac{u t}{4 s\left(Q^{2}-t\right)}+F\left(Q^{2}, 0\right) \frac{Q^{2}\left(3 Q^{2}-t\right)}{8\left(Q^{2}-t\right)^{2}} \\
& +\frac{F\left(Q^{2}, 0\right)+G\left(Q^{2}, 0\right)}{8} \frac{Q^{2}\left(\left(Q^{2}-t\right)^{2}+u t\right)}{s\left(Q^{2}-t\right)^{2}}, \\
C_{2 R R}^{018}\left(s, t, Q^{2}\right)= & -\frac{u t}{s\left(Q^{2}-t\right)^{2}} C_{21}\left(0, Q^{2}, t\right)+\frac{C_{22}\left(0, Q^{2}, t\right)}{4}+\frac{\beta_{-}^{2}(t, s, u)}{4} C_{22}\left(Q^{2}, 0, t\right) \\
& -\frac{\beta_{-}(t, s, u)}{2} C_{23}\left(0, Q^{2}, t\right) \\
= & C_{2 P P}^{018}\left(s, t, Q^{2}\right)+\left[F\left(Q^{2}, t\right)\left(2\left(Q^{2}-u\right)+s\right)-G\left(Q^{2}, t\right) s\right] \frac{Q^{2}-t}{4 s^{2}}, \\
C_{2 T T}^{018}\left(s, t, Q^{2}\right)= & -\frac{s}{u t} C_{21}\left(0, Q^{2}, t\right)+C_{22}\left(Q^{2}, 0, t\right)=-\frac{H\left(Q^{2}, t\right)}{D-2} \frac{s\left(Q^{2}-t\right)}{4 u t}-\frac{F\left(Q^{2}, t\right)}{2}, \\
C_{2 P R}^{018}\left(s, t, Q^{2}\right)= & -\frac{u t}{s\left(Q^{2}-t\right)^{2}} C_{21}\left(0, Q^{2}, t\right)+\frac{C_{22}\left(0, Q^{2}, t\right)}{4} \\
& -\frac{\alpha_{-}(t, s, u) \beta-(t, s, u)}{4} C_{22}\left(Q^{2}, 0, t\right)-\frac{\alpha-(t, u, s)}{2} C_{23}\left(0, Q^{2}, t\right) \\
C_{2 R T}^{018}\left(s, t, Q^{2}\right)= & \frac{C_{21}\left(0, Q^{2}, t\right)}{Q^{2}-t}+\frac{\beta_{-}(t, s, u)}{2} C_{22}\left(Q^{2}, 0, t\right)-\frac{C_{23}\left(0, Q^{2}, t\right)}{2} \\
= & C_{2 P T}^{018}\left(s, t, Q^{2}\right)-F\left(Q^{2}, t\right) \frac{Q^{2}-t}{2 s} \cdot\left(\mathrm{A}, t, Q^{2}\right)+\left[F\left(Q^{2}, t\right)\left(2 Q^{2}+3 s\right)-G\left(Q^{2}, t\right) s\right] \frac{Q^{2}-t}{8 s^{2}}, \\
C_{2 P T}^{018}\left(s, t, Q^{2}\right)= & \frac{C_{21}\left(0, Q^{2}, t\right)}{Q^{2}-t}-\frac{\alpha_{-}(t, s, u)}{2} C_{22}\left(Q^{2}, 0, t\right)-\frac{C_{23}\left(0, Q^{2}, t\right)}{2} \\
& H\left(Q^{2}, t\right) \\
\hline & F\left(Q^{2}, t\right)\left(2 Q^{2}+3 s\right)-G\left(Q^{2}, t\right) s \\
&
\end{aligned}
$$

Loop integrals $C^{\mu \nu ; 019}$ and $C^{\mu \nu ; 023}$. Sets of the scalar functions:

$$
\begin{aligned}
& C_{2 g}^{019}\left(s, u, Q^{2}\right), C_{2 P P}^{019}\left(s, u, Q^{2}\right), C_{2 R R}^{019}\left(s, u, Q^{2}\right), C_{2 T T}^{019}\left(s, u, Q^{2}\right) \\
& C_{2 P R}^{019}\left(s, u, Q^{2}\right), C_{2 P T}^{019}\left(s, u, Q^{2}\right), C_{2 R T}^{019}\left(s, u, Q^{2}\right) \\
& C_{2 g}^{023}\left(s, t, Q^{2}\right), C_{2 P P}^{023}\left(s, t, Q^{2}\right), C_{2 R R}^{023}\left(s, t, Q^{2}\right), C_{2 T T}^{023}\left(s, t, Q^{2}\right) \\
& C_{2 P R}^{023}\left(s, t, Q^{2}\right), C_{2 P T}^{023}\left(s, t, Q^{2}\right), C_{2 R T}^{023}\left(s, t, Q^{2}\right)
\end{aligned}
$$


Relations:

$$
\left\{\begin{array}{l}
C_{2 g}^{019}\left(s, u, Q^{2}\right)=+C_{2 g}^{023}\left(s, u, Q^{2}\right), \\
C_{2 P P}^{019}\left(s, u, Q^{2}\right)=+C_{2 P P}^{023}\left(s, u, Q^{2}\right), \\
C_{2 R R}^{019}\left(s, u, Q^{2}\right)=+C_{2 R R}^{023}\left(s, u, Q^{2}\right), \\
C_{2 T T}^{019}\left(s, u, Q^{2}\right)=+C_{2 T T}^{023}\left(s, u, Q^{2}\right), \\
C_{2 P R}^{019}\left(s, u, Q^{2}\right)=-C_{2 P R}^{023}\left(s, u, Q^{2}\right), \\
C_{2 P T}^{019}\left(s, u, Q^{2}\right)=+C_{2 P T}^{023}\left(s, u, Q^{2}\right), \\
C_{2 R T}^{019}\left(s, u, Q^{2}\right)=-C_{2 R T}^{023}\left(s, u, Q^{2}\right) .
\end{array}\right.
$$

Explicit results:

$$
\begin{aligned}
C_{2 g}^{019}\left(s, u, Q^{2}\right)= & C_{21}(0,0, u)=\frac{F(u, 0)+G_{0}(u, 0)}{D-2} \frac{u}{4} \\
C_{2 P P}^{019}\left(s, u, Q^{2}\right)= & -\frac{t}{s u} C_{21}(0,0, u)+\frac{s^{2}+\left(Q^{2}-s\right)^{2}}{4 s^{2}} C_{22}(0,0, u)-\frac{Q^{2}-s}{2 s} C_{23}(0,0, u) \\
= & -\frac{F(u, 0)+G(u, 0)}{D-2} \frac{t}{4 s}-F(u, 0) \frac{\left(Q^{2}-2 s\right)^{2}}{8 s^{2}} \\
& +[G(u, 0)-F(u, 0)] \frac{Q^{2}-s}{8 s}, \\
C_{2 R R}^{019}\left(s, u, Q^{2}\right)= & -\frac{t}{s u} C_{21}(0,0, u)+\frac{s^{2}+(u-t)^{2}}{4 s^{2}} C_{22}(0,0, u)+\frac{u-t}{2 s} C_{23}(0,0, u) \\
= & C_{2 P P}^{019}\left(s, u, Q^{2}\right)-[F(u, 0)+G(u, 0)] \frac{u}{4 s}+F(u, 0) \frac{u t}{2 s^{2}}, \\
C_{2 T T}^{019}\left(s, u, Q^{2}\right)= & -\frac{s}{u t} C_{21}(0,0, u)+C_{22}(0,0, u)=-\frac{F(u, 0)+G(u, 0)}{D} \frac{s}{4 t}-\frac{F(u, 0)}{2} \\
C_{2 P R}^{019}\left(s, u, Q^{2}\right)= & -\frac{t}{s u} C_{21}(0,0, u)+\frac{s^{2}+t^{2}-u^{2}}{4 s u} C_{22}(0,0, u)-\frac{t}{2 s} C_{23}(0,0, u) \\
= & C_{2 P P}^{019}\left(s, u, Q^{2}\right)-[F(u, 0)+G(u, 0)] \frac{u}{8 s} \\
& -F(u, 0)\left[\frac{\left(Q^{2}-s\right)^{2}+s^{2}}{8 s u}-\frac{Q^{4}}{8 s^{2}}\right] \\
C_{2 R T}^{019}\left(s, u, Q^{2}\right)= & \frac{1}{u} C_{21}(0,0, u)+\frac{u-t}{2 s} C_{22}(0,0, u)+\frac{1}{2} C_{23}(0,0, u) \\
= & C_{2 P T}^{019}\left(s, u, Q^{2}\right)-F(u, 0) \frac{u}{2 s} \cdot \\
C_{2 P T}^{019}\left(s, u, Q^{2}\right)= & \frac{1}{u} C_{21}(0,0, u)-\frac{Q^{2}-s}{2 s} C_{22}(0,0, u)+\frac{1}{2} C_{23}(0,0, u) \\
= & \frac{G(u, 0)+F(u, 0)}{4-D} \frac{4-2}{8}+F(u, 0) \frac{Q^{2}-s}{4 s}, \\
& (\mathrm{~A} .59)
\end{aligned}
$$

Loop integrals $C^{\mu \nu ; 035}$ and $C^{\mu \nu ; 069}$. Sets of the scalar functions:

$$
\begin{aligned}
& C_{2 g}^{035}\left(s, u, Q^{2}\right), C_{2 P P}^{035}\left(s, u, Q^{2}\right), C_{2 R R}^{035}\left(s, u, Q^{2}\right), C_{2 T T}^{035}\left(s, u, Q^{2}\right), \\
& C_{2 P R}^{035}\left(s, u, Q^{2}\right), C_{2 P T}^{035}\left(s, u, Q^{2}\right), C_{2 R T}^{035}\left(s, u, Q^{2}\right), \\
& C_{2 g}^{069}\left(s, t, Q^{2}\right), C_{2 P P}^{069}\left(s, t, Q^{2}\right), C_{2 R R}^{069}\left(s, t, Q^{2}\right), C_{2 T T}^{069}\left(s, t, Q^{2}\right), \\
& C_{2 P R}^{069}\left(s, t, Q^{2}\right), C_{2 P T}^{069}\left(s, t, Q^{2}\right), C_{2 R T}^{069}\left(s, t, Q^{2}\right) .
\end{aligned}
$$


Relations:

$$
\left\{\begin{array}{l}
C_{2 g}^{035}\left(s, u, Q^{2}\right)=+C_{2 g}^{069}\left(s, u, Q^{2}\right), \\
C_{2 P P}^{035}\left(s, u, Q^{2}\right)=+C_{2 P P}^{069}\left(s, u, Q^{2}\right), \\
C_{2 R R}^{035}\left(s, u, Q^{2}\right)=+C_{2 R R}^{069}\left(s, u, Q^{2}\right), \\
C_{2 T T}^{035}\left(s, u, Q^{2}\right)=+C_{2 T T}^{069}\left(s, u, Q^{2}\right), \\
C_{2 P R}^{035}\left(s, u, Q^{2}\right)=-C_{2 P R}^{069}\left(s, u, Q^{2}\right), \\
C_{2 P T}^{035}\left(s, u, Q^{2}\right)=+C_{2 P T}^{069}\left(s, u, Q^{2}\right), \\
C_{2 R T}^{035}\left(s, u, Q^{2}\right)=-C_{2 R T}^{069}\left(s, u, Q^{2}\right) .
\end{array}\right.
$$

Explicit results:

$$
\begin{aligned}
& C_{2 g}^{035}\left(s, u, Q^{2}\right)=C_{21}(0, u, 0)=\frac{F(u, 0)+G(u, 0)}{D-2} \frac{u}{4}, \\
& C_{2 P P}^{035}\left(s, u, Q^{2}\right)=-\frac{t}{s u} C_{21}(0, u, 0)+\frac{\left(Q^{2}-s\right)^{2}}{4 s^{2}} C_{22}(0, u, 0)+\frac{\left(Q^{2}-2 s\right)^{2}}{4 s^{2}} C_{22}(u, 0,0) \\
& -\frac{\left(Q^{2}-s\right)\left(Q^{2}-2 s\right)}{2 s^{2}} C_{23}(0, u, 0) \\
& =-\frac{F(u, 0)}{2 s^{2}}\left[\frac{\left(Q^{2}-s\right)^{2}}{D-4}+\frac{Q^{4}}{4}\right]-\frac{F(u, 0)+G(u, 0)}{D-2} \frac{t}{4 s} \\
& +[G(u, 0)-F(u, 0)] \frac{Q^{2}-s}{8 s}, \\
& C_{2 R R}^{035}\left(s, u, Q^{2}\right)=-\frac{t}{s u} C_{21}(0, u, 0)+\frac{(u-t)^{2}}{4 s^{2}} C_{22}(0, u, 0)+\frac{\left(Q^{2}-2 t\right)^{2}}{4 s^{2}} C_{22}(u, 0,0) \\
& -\frac{\left(Q^{2}-2 t\right)(u-z)}{2 s^{2}} C_{23}(0, u, 0) \\
& =C_{2 P P}^{035}\left(s, u, Q^{2}\right)+F(u, 0) \frac{2 u t}{s^{2}}\left[\frac{1}{D-4}+\frac{Q^{2}-u}{4 t}\right] \\
& +[F(u, 0)-G(u, 0)] \frac{u}{4 s}, \\
& C_{2 T T}^{035}\left(s, u, Q^{2}\right)=-\frac{s}{u t} C_{21}(0, u, 0)+C_{22}(0, u, 0)+C_{22}(u, 0,0)-2 C_{23}(0, u, 0) \\
& =-F(u, 0) \frac{D}{2(D-4)}-\frac{F(u, 0)+G(u, 0)}{D-2} \frac{s}{4 t}, \\
& C_{2 P R}^{035}\left(s, u, Q^{2}\right)=-\frac{t}{s u} C_{21}(0, u, 0)-\frac{(u-t)\left(Q^{2}-s\right)}{4 s^{2}} C_{22}(0, u, 0) \\
& -\frac{\left(Q^{2}-2 s\right)\left(Q^{2}-2 t\right)}{4 s^{2}} C_{22}(u, 0,0)+\frac{(s-t) t+u^{2}}{2 s^{2}} C_{23}(0, u, 0) \\
& =C_{2 P P}^{035}\left(s, u, Q^{2}\right)+F(u, 0) \frac{u}{s^{2}}\left[\frac{Q^{2}-s}{D-4}+\frac{Q^{2}}{4}\right] \\
& +[F(u, 0)-G(u, 0)] \frac{u}{8 s}, \\
& C_{2 P T}^{035}\left(s, u, Q^{2}\right)=\frac{1}{u} C_{21}(0, u, 0)-\frac{Q^{2}-s}{2 s} C_{22}(0, u, 0)-\frac{Q^{2}-2 s}{2 s} C_{22}(u, 0,0) \\
& +\frac{2 Q^{2}-3 s}{2 s} C_{23}(0, u, 0) \\
& =F(u, 0) \frac{1}{s}\left[\frac{Q^{2}-s}{D-4}+\frac{Q^{2}+s}{4}\right]+\frac{F(u, 0)+G(u, 0)}{8} \frac{4-D}{D-2},
\end{aligned}
$$




$$
\begin{aligned}
C_{2 R T}^{035}\left(s, u, Q^{2}\right)= & \frac{1}{u} C_{21}(0, u, 0)+\frac{u-t}{2 s} C_{22}(0, u, 0)+\frac{Q^{2}-2 t}{2 s} C_{22}(u, 0,0) \\
& -\frac{2(u-t)+s}{2 s} C_{23}(0, u, 0) \\
= & C_{2 P T}^{035}\left(s, u, Q^{2}\right)-F(u, 0) \frac{u}{2 s} \frac{D}{D-4} .
\end{aligned}
$$

Loop integrals $C^{\mu \nu ; 038}$ and $C^{\mu \nu ; 049}$. Sets of the scalar functions:

$$
\begin{aligned}
& C_{2 g}^{038}\left(s, u, Q^{2}\right), C_{2 P P}^{038}\left(s, u, Q^{2}\right), C_{2 R R}^{038}\left(s, u, Q^{2}\right), C_{2 T T}^{038}\left(s, u, Q^{2}\right), \\
& C_{2 P R}^{038}\left(s, u, Q^{2}\right), C_{2 P T}^{038}\left(s, u, Q^{2}\right), C_{2 R T}^{038}\left(s, u, Q^{2}\right), \\
& C_{2 g}^{049}\left(s, t, Q^{2}\right), C_{2 P P}^{049}\left(s, t, Q^{2}\right), C_{2 R R}^{049}\left(s, t, Q^{2}\right), C_{2 T T}^{049}\left(s, t, Q^{2}\right), \\
& C_{2 P R}^{049}\left(s, t, Q^{2}\right), C_{2 P T}^{049}\left(s, t, Q^{2}\right), C_{2 R T}^{049}\left(s, t, Q^{2}\right) .
\end{aligned}
$$

Relations:

$$
\left\{\begin{array}{l}
C_{2 g}^{038}\left(s, u, Q^{2}\right)=+C_{2 g}^{049}\left(s, u, Q^{2}\right), \\
C_{2 P P}^{039}\left(s, u, Q^{2}\right)=+C_{2 P P}^{049}\left(s, u, Q^{2}\right), \\
C_{2 R R}^{038}\left(s, u, Q^{2}\right)=+C_{2 R R}^{049}\left(s, u, Q^{2}\right), \\
C_{2 T T}^{038}\left(s, u, Q^{2}\right)=+C_{2 T T}^{049}\left(s, u, Q^{2}\right), \\
C_{2 P R}^{038}\left(s, u, Q^{2}\right)=+C_{2 P R}^{049}\left(s, u, Q^{2}\right), \\
C_{2 P T}^{038}\left(s, u, Q^{2}\right)=+C_{2 P T}^{049}\left(s, u, Q^{2}\right), \\
C_{2 R T}^{038}\left(s, u, Q^{2}\right)=+C_{2 R T}^{049}\left(s, u, Q^{2}\right) .
\end{array}\right.
$$

Explicit results:

$$
\begin{aligned}
C_{2 g}^{038}\left(s, u, Q^{2}\right)= & C_{21}\left(0, Q^{2}, s\right)\left(s, u, Q^{2}\right)=\frac{H\left(Q^{2}, s\right)}{D-2} \frac{Q^{2}-s}{4}, \\
C_{2 P P}^{038}\left(s, u, Q^{2}\right)= & \frac{\left(Q^{2}-s\right)^{2}}{4 s^{2}}\left[C_{22}\left(0, Q^{2}, s\right)+C_{22}\left(Q^{2}, 0, s\right)-2 C_{23}\left(0, Q^{2}, s\right)\right] \\
= & -F\left(Q^{2}, s\right) \frac{Q^{4}}{2 s^{2}} \frac{D-3}{D-4}+\left[G\left(Q^{2}, 0\right) s+3 F\left(Q^{2}, 0\right) Q^{2}\right] \frac{Q^{2}}{8 s^{2}}, \\
C_{2 R R}^{038}\left(s, u, Q^{2}\right)= & -\frac{4 t u}{s\left(Q^{2}-s\right)^{2}} C_{21}\left(0, Q^{2}, s\right)+\frac{(t-u)^{2}}{4 s^{2}} C_{22}\left(0, Q^{2}, s\right) \\
& +\frac{(t-u)^{2}\left(Q^{2}+s\right)^{2}}{4 s^{2}\left(Q^{2}-s\right)^{2}} C_{22}\left(Q^{2}, 0, s\right)-\frac{(t-u)^{2}\left(Q^{2}+s\right)}{2 s^{2}\left(Q^{2}-s\right)} C_{23}\left(0, Q^{2}, s\right) \\
= & -F\left(Q^{2}, s\right) \frac{(u-t)^{2}}{2 s^{2}\left(Q^{2}-s\right)^{2}}\left[\frac{Q^{4}}{D-4}+\left(Q^{2}-s\right)^{2}\right]-\frac{H\left(Q^{2}, s\right)}{D-2} \frac{u t}{s\left(Q^{2}-s\right)} \\
& -\left[G\left(Q^{2}, 0\right) s+F\left(Q^{2}, 0\right)\left(2 s-3 Q^{2}\right)\right] \frac{(u-t)^{2} Q^{2}}{8 s^{2}\left(Q^{2}-s\right)^{2}}, \\
C_{2 T T}^{038}\left(s, u, Q^{2}\right)= & -\frac{s(u-t)^{2}}{u t\left(Q^{2}-s\right)^{2}} C_{21}\left(0, Q^{2}, s\right)+C_{22}\left(0, Q^{2}, s\right)+\frac{\left(Q^{2}+s\right)^{2}}{\left(Q^{2}-s\right)^{2}} C_{22}\left(Q^{2}, 0, s\right) \\
& -2 \frac{Q^{2}+s}{Q^{2}-s} C_{23}\left(0, Q^{2}, s\right)=\frac{4 s^{2}}{(u-t)^{2}}\left[C_{2 R R}^{038}+\frac{4 u t-(u-t)^{2}}{4 s t u} C_{2 g}^{038}\right], \\
& \frac{t-u}{4 s^{2}}\left[\left(Q^{2}-s\right) C_{22}\left(0, Q^{2}, s\right)+\left(Q^{2}+s\right) C_{22}\left(Q^{2}, 0, s\right)-2 Q^{2} C_{23}\left(0, Q^{2}, s\right)\right] \\
C_{2 P R}^{038}\left(s, u, Q^{2}\right) & \frac{t-u}{Q^{2}-s}\left[C_{2 P P}^{038}\left(s, u, Q^{2}\right)-\frac{\left(3 Q^{2}+s\right) F\left(Q^{2}, s\right)-\left(Q^{2}-s\right) G\left(Q^{2}, s\right)}{8 s}\right],
\end{aligned}
$$




$$
\begin{aligned}
C_{2 P T}^{038}\left(s, u, Q^{2}\right)= & \frac{2 s}{u-t} C_{2 P R}^{038}\left(s, u, Q^{2}\right) \\
C_{2 R T}^{038}\left(s, u, Q^{2}\right)= & \frac{u-t}{2 s}\left[\frac{4 s}{\left(Q^{2}-s\right)^{2}} C_{21}\left(0, Q^{2}, s\right)+C_{22}\left(0, Q^{2}, s\right)\right. \\
& \left.+\frac{\left(Q^{2}+s\right)^{2}}{\left(Q^{2}-s\right)^{2}} C_{22}\left(Q^{2}, 0, s\right)-\frac{2\left(Q^{2}+s\right)}{Q^{2}-s} C_{23}\left(0, Q^{2}, s\right)\right] \\
= & \frac{2 s}{u-t}\left[C_{2 P R}^{038}\left(s, u, Q^{2}\right)+\frac{1}{s} C_{2 g}^{038}\right] .
\end{aligned}
$$

Loop integrals $C^{\mu \nu ; 046}$ and $C^{\mu \nu ; 058}$. Sets of the scalar functions:

$$
\begin{aligned}
& C_{2 g}^{046}\left(s, t, Q^{2}\right), C_{2 P P}^{046}\left(s, t, Q^{2}\right), C_{2 R R}^{046}\left(s, t, Q^{2}\right), C_{2 T T}^{046}\left(s, t, Q^{2}\right) \\
& C_{2 P R}^{046}\left(s, t, Q^{2}\right), C_{2 P T}^{046}\left(s, t, Q^{2}\right), C_{2 R T}^{046}\left(s, t, Q^{2}\right) \\
& C_{2 g}^{058}\left(s, u, Q^{2}\right), C_{2 P P}^{058}\left(s, u, Q^{2}\right), C_{2 R R}^{058}\left(s, u, Q^{2}\right), C_{2 T T}^{058}\left(s, u, Q^{2}\right), \\
& C_{2 P R}^{058}\left(s, u, Q^{2}\right), C_{2 P T}^{058}\left(s, u, Q^{2}\right), C_{2 R T}^{058}\left(s, u, Q^{2}\right) .
\end{aligned}
$$

Relations:

$$
\left\{\begin{array}{l}
C_{2 g}^{046}\left(s, t, Q^{2}\right)=+C_{2 g}^{058}\left(s, t, Q^{2}\right), \\
C_{2 P P}^{046}\left(s, t, Q^{2}\right)=+C_{2 P P}^{058}\left(s, t, Q^{2}\right), \\
C_{2 R R}^{046}\left(s, t, Q^{2}\right)=+C_{2 R R}^{058}\left(s, t, Q^{2}\right), \\
C_{2 T T}^{046}\left(s, t, Q^{2}\right)=+C_{2 T T}^{058}\left(s, t, Q^{2}\right), \\
C_{2 P R}^{046}\left(s, t, Q^{2}\right)=-C_{2 P R}^{058}\left(s, t, Q^{2}\right), \\
C_{2 P T}^{046}\left(s, t, Q^{2}\right)=+C_{2 P T}^{058}\left(s, t, Q^{2}\right), \\
C_{2 R T}^{046}\left(s, t, Q^{2}\right)=-C_{2 R T}^{058}\left(s, t, Q^{2}\right) .
\end{array}\right.
$$

Explicit results:

$$
\begin{aligned}
C_{2 g}^{046}\left(s, t, Q^{2}\right)= & C_{21}\left(Q^{2}, t, 0\right)=\frac{H\left(Q^{2}, t\right)}{D-2} \frac{Q^{2}-t}{4} \\
C_{2 P P}^{046}\left(s, t, Q^{2}\right)= & -\frac{u t}{s\left(Q^{2}-t\right)^{2}} C_{21}\left(Q^{2}, t, 0\right)+\frac{\alpha_{+}^{2}(t, s, u)}{4} C_{22}\left(Q^{2}, t, 0\right) \\
& +\frac{\gamma_{+}^{2}(t, u, s)}{4} C_{22}\left(t, Q^{2}, 0\right)-\frac{\alpha_{+}(t, s, u) \gamma_{+}(t, u, s)}{2} C_{23}\left(Q^{2}, t, 0\right) \\
= & -\frac{F\left(Q^{2}, t\right)}{2 s^{2}}\left[\frac{\left(\left(Q^{2}-t\right)^{2}+u t\right)^{2}}{\left(Q^{2}-t\right)^{2}(D-4)}+\frac{Q^{4}}{4}\right]-\frac{H\left(Q^{2}, t\right)}{D-2} \frac{u t}{4 s\left(Q^{2}-t\right)} \\
& -F\left(Q^{2}, t\right) \frac{Q^{2}\left(2 Q^{2}+s\right)}{8 s\left(Q^{2}-t\right)}+\left[G\left(Q^{2}, 0\right)-F\left(Q^{2}, 0\right)\right] \frac{Q^{2}\left(\left(Q^{2}-t\right)^{2}+u t\right)}{8 s\left(Q^{2}-t\right)^{2}}, \\
C_{2 R R}^{046}\left(s, t, Q^{2}\right)= & -\frac{u t}{s\left(Q^{2}-t\right)^{2}} C_{21}\left(Q^{2}, t, 0\right)+\frac{\beta_{+}^{2}(t, s, u)}{4} C_{22}\left(Q^{2}, t, 0\right) \\
& +\frac{\gamma_{+}^{2}(t, s, u)}{4} C_{22}\left(t, Q^{2}, 0\right)-\frac{\beta_{+}(t, s, u) \gamma_{+}(t, s, u)}{2} C_{23}\left(Q^{2}, t, 0\right) \\
= & C_{2 P P}^{046}\left(s, t, Q^{2}\right)+2 F\left(Q^{2}, t\right) \frac{u t}{s^{2}}\left[\frac{1}{D-4}+\frac{Q^{2}-t}{4 u}\right] \\
& +\frac{Q^{2}}{4 s}\left[3 F\left(Q^{2}, 0\right)-G\left(Q^{2}, 0\right)\right]
\end{aligned}
$$




$$
\begin{aligned}
& C_{2 T T}^{046}\left(s, t, Q^{2}\right)=-\frac{s}{u t} C_{21}\left(Q^{2}, t, 0\right)+\frac{\left(Q^{2}-t\right)^{2}}{\left(Q^{2}+t\right)^{2}}\left[C_{22}\left(Q^{2}, t, 0\right)+C_{22}\left(t, Q^{2}, 0\right)\right. \\
& \left.-2 C_{23}\left(Q^{2}, t, 0\right)\right]=-F\left(Q^{2}, t\right) \frac{D}{2(D-4)}-\frac{H\left(Q^{2}, t\right)}{D-2} \frac{s\left(Q^{2}-t\right)}{4 u t}, \\
& C_{2 P R}^{046}\left(s, t, Q^{2}\right)=-\frac{u t}{s\left(Q^{2}-t\right)^{2}} C_{21}\left(Q^{2}, t, 0\right)-\frac{\alpha_{+}(t, s, u) \beta_{+}(t, s, u)}{4} C_{22}\left(Q^{2}, t, 0\right) \\
& -\frac{\gamma_{+}(t, s, u) \gamma_{+}(t, u, s)}{2} C_{22}\left(t, Q^{2}, 0\right)+\frac{\sigma_{+}(t, u, s)}{2} C_{23}\left(Q^{2}, t, 0\right) \\
& =C_{2 P P}^{046}\left(s, t, Q^{2}\right)+\frac{F\left(Q^{2}, t\right)}{s^{2}}\left[\frac{\left(Q^{2}-t\right)^{2}+u t}{\left(Q^{2}-t\right)^{2}(D-4)}+\frac{Q^{2}\left(Q^{2}-2 t\right)}{4}\right] \\
& +-\frac{H\left(Q^{2}, t\right)}{D-2} \frac{u t}{4 s\left(Q^{2}-t\right)}+\left[3 F\left(Q^{2}, 0\right)-G\left(Q^{2}, 0\right)\right] \frac{Q^{2}}{8 s}, \\
& C_{2 P T}^{046}\left(s, t, Q^{2}\right)=\frac{1}{Q^{2}-t} C_{21}\left(Q^{2}, t, 0\right) \\
& -\frac{Q^{2}-t}{Q^{2}+t}\left[\frac{\alpha_{+}(t, s, u)}{2}\left(C_{22}\left(Q^{2}, t, 0\right)-C_{23}\left(Q^{2}, t, 0\right)\right)\right. \\
& \left.+\frac{\gamma_{+}(t, u, s)}{2}\left(C_{22}\left(t, Q^{2}, 0\right)-C_{23}\left(Q^{2}, t, 0\right)\right)\right] \\
& =F\left(Q^{2}, t\right) \frac{Q^{2}}{s}\left[\frac{\left(Q^{2}-t\right)^{2}+u t}{Q^{2}\left(Q^{2}-t\right)(D-4)}+\frac{1}{4}\right] \\
& +\frac{H\left(Q^{2}, t\right)}{4(D-2)}+\left[3 F\left(Q^{2}, 0\right)-G\left(Q^{2}, 0\right)\right] \frac{Q^{2}}{8\left(Q^{2}-t\right)}, \\
& C_{2 R T}^{046}\left(s, t, Q^{2}\right)=\frac{1}{Q^{2}-t} C_{21}\left(Q^{2}, t, 0\right) \\
& +\frac{Q^{2}-t}{Q^{2}+t}\left[\frac{\beta_{+}(t, s, u)}{2}\left(C_{22}\left(Q^{2}, t, 0\right)-C_{23}\left(Q^{2}, t, 0\right)\right)\right. \\
& \left.+\frac{\gamma_{+}(t, s, u)}{2}\left(C_{22}\left(t, Q^{2}, 0\right)-C_{23}\left(Q^{2}, t, 0\right)\right)\right] \\
& =C_{2 P T}^{046}\left(s, t, Q^{2}\right)-F\left(Q^{2}, t\right) \frac{Q^{2}-t}{2 s} \frac{D}{D-4} \text {. }
\end{aligned}
$$

Loop integrals $C^{\mu \nu ; 078}$ and $C^{\mu \nu ; 079}$. Sets of the scalar functions:

$$
\begin{aligned}
& C_{2 g}^{078}\left(s, u, Q^{2}\right), C_{2 P P}^{068}\left(s, u, Q^{2}\right), C_{2 R R}^{078}\left(s, u, Q^{2}\right), C_{2 T T}^{078}\left(s, u, Q^{2}\right) \\
& C_{2 P R}^{078}\left(s, u, Q^{2}\right), C_{2 P T}^{078}\left(s, u,{ }^{2}\right), C_{2 R T}^{078}\left(s, u, Q^{2}\right) \\
& C_{2 g}^{079}\left(s, u, Q^{2}\right), C_{2 P P}^{079}\left(s, u, Q^{2}\right), C_{2 R R}^{079}\left(s, u, Q^{2}\right), C_{2 T T}^{079}\left(s, u, Q^{2}\right) \\
& C_{2 P R}^{079}\left(s, u, Q^{2}\right), C_{2 P T}^{079}\left(s, u, Q^{2}\right), C_{2 R T}^{079}\left(s, u, Q^{2}\right)
\end{aligned}
$$


Relations:

$$
\begin{cases}C_{2 K}^{078}\left(s, u, Q^{2}\right) & =C_{2 K}^{079}\left(s, u, Q^{2}\right), \quad K=g, R R, T T, R T \\ C_{2 P P}^{078}\left(s, u, Q^{2}\right)+C_{1 P}^{078}\left(s, u, Q^{2}\right) & =C_{2 P P}^{078}\left(s, u, Q^{2}\right)+C_{1 P}^{079}\left(s, u, Q^{2}\right) \\ C_{2 P R}^{078}\left(s, u, Q^{2}\right)+\frac{1}{2} C_{1 R}^{078}\left(s, u, Q^{2}\right) & =C_{2 P R}^{079}\left(s, u, Q^{2}\right)+\frac{1}{2} C_{1 R}^{079}\left(s, u, Q^{2}\right) \\ C_{2 P T}^{078}\left(s, u, Q^{2}\right)+\frac{1}{2} C_{1 T}^{078}\left(s, u, Q^{2}\right) & =C_{2 P T}^{079}\left(s, u, Q^{2}\right)+\frac{1}{2} C_{1 T}^{079}\left(s, u, Q^{2}\right)\end{cases}
$$

Explicit results:

$$
\begin{aligned}
C_{2 g}^{078}\left(s, u, Q^{2}\right)= & C_{21}\left(s, Q^{2}, 0\right)=\frac{H\left(Q^{2}, s\right)}{D-2} \frac{\left(Q^{2}-s\right)}{4}, \\
C_{2 P P}^{078}\left(s, u, Q^{2}\right)= & \frac{\left(Q^{2}-s\right)^{4}}{4 s^{2}\left(Q^{2}+s\right)^{2}} C_{22}\left(Q^{2}, s, 0\right) \\
= & -\frac{F\left(Q^{2}, s\right)}{2} \frac{D-3}{D-4}+\frac{Q^{2}}{8 s}\left[G\left(Q^{2}, 0\right)-\frac{Q^{2}+4 s}{s} F\left(Q^{2}, 0\right)\right], \\
C_{2 R R}^{078}\left(s, u, Q^{2}\right)= & \frac{(u-t)^{2}}{4 s^{2}}\left[-\frac{16 s u t}{\left(Q^{2}-s\right)^{2}(u-t)^{2}} C_{21}\left(s, Q^{2}, 0\right)\right. \\
& \left.+\frac{4 s^{2}}{\left(Q^{2}+s\right)^{2}} C_{22}\left(s, Q^{2}, 0\right)+C_{22}\left(Q^{2}, s, 0\right)-\frac{4 s}{Q^{2}+s} C_{23}\left(s, Q^{2}, 0\right)\right] \\
= & -\frac{F\left(Q^{2}, s\right)}{D-4} \frac{(u-t)^{2}}{2\left(Q^{2}-s\right)^{2}}-\frac{H\left(Q^{2}, s\right)}{D-2} \frac{u t}{s\left(Q^{2}-s\right)} \\
& -\frac{Q^{2}(u-t)^{2}}{8 s^{2}\left(Q^{2}-s\right)^{2}}\left[G\left(Q^{2}, 0\right) s+F\left(Q^{2}, 0\right)\left(Q^{2}-2 s\right)\right], \\
C_{2 T T}^{078}\left(s, u, Q^{2}\right)= & -\frac{s(u-t)^{2}}{u t\left(Q^{2}-s\right)^{2}} C_{21}\left(s, Q^{2}, 0\right)+\frac{4 s^{2}}{\left(Q^{2}+s\right)^{2}} C_{22}\left(s, Q^{2}, 0\right) \\
& +C_{22}\left(Q^{2}, s, 0\right)-\frac{4 s}{Q^{2}+s} C_{23}\left(s, Q^{2}, 0\right) \\
C_{2 R T}^{078}\left(s, u, Q^{2}\right)= & \frac{u-t}{2 s}\left[\frac{4 s}{\left(Q^{2}-s\right)^{2}} C_{21}\left(s, Q^{2}, 0\right)+\frac{4 s^{2}}{\left(Q^{2}+s\right)^{2}} C_{22}\left(s, Q^{2}, 0\right)\right. \\
C_{2 P T}^{078}\left(s, u, Q^{2}\right)= & \frac{2 s 8}{u-t} C_{2 P R}^{078}\left(s, u, Q^{2}\right)+\frac{4 u t-(u-t)^{2}}{4 s t u} C_{2 g}^{078}\left(s, u, Q^{2}\right], \\
C_{2 P R}^{078}\left(s, u, Q^{2}\right)= & \frac{(t-u)\left(Q^{2}-s\right)^{2}}{4 s^{2}\left(Q^{2}+s\right)^{2}}\left[\left(Q^{2}+s\right) C_{22}\left(Q^{2}, s, 0\right)-2 s C_{23}\left(s, Q^{2}, 0\right)\right] \\
= & \frac{F\left(Q^{2}, s\right)}{D-4} \frac{u-t}{2\left(Q^{2}-s\right)}+\frac{Q^{2}\left(Q^{2}+s\right)(u-t)}{8\left(Q^{2}-s\right) s^{2}} F\left(Q^{2}, 0\right), \\
& \left.C_{23}\left(s, Q^{2}, 0\right)\right] \\
& \\
&
\end{aligned}
$$




\section{B Box integrals}

\section{B.1 Scalar box integral}

First, we discuss calculation of the scalar box integral $D_{0}\left(s, u, Q^{2}\right)$. After $\alpha$ parametrization and integration over loop momentum the integral $D_{0}\left(s, u, Q^{2}\right)$ takes the form

$$
\begin{aligned}
D_{0}\left(s, u, Q^{2}\right)= & \left(\pi \mu^{-2}\right)^{\frac{D}{2}-2} \Gamma\left(4-\frac{D}{2}\right) \int_{0}^{\infty} d \alpha_{1} \ldots \int_{0}^{\infty} d \alpha_{4} \\
& \times \frac{\delta\left(1-\sum_{i=1}^{4} \alpha_{i}\right)\left(\sum_{i=1}^{4} \alpha_{i}\right)^{4-D}}{\left(-s \alpha_{3}\left(\alpha_{2}+\alpha_{4}\right)-u \alpha_{4}\left(\alpha_{1}+\alpha_{3}\right)-t \alpha_{3} \alpha_{4}\right)^{4-\frac{D}{2}}} .
\end{aligned}
$$

Next we apply the change of variables ( $\alpha$ parameters) using the trick from [24]:

$$
\alpha_{1}=\eta_{1}\left(1-\xi_{1}\right), \quad \alpha_{2}=\eta_{2}\left(1-\xi_{2}\right), \quad \alpha_{3}=\eta_{1} \xi_{1}, \quad \alpha_{4}=\eta_{2} \xi_{2} .
$$

Next we take into account that the Jacobian of such the change of variables is $J=\eta_{1} \eta_{2}$, integrate over $\eta_{2}$ using $\delta$ function, and integrate over $\eta_{1}$ using the integral representation for beta function (A.5).

After these tricks the box integral takes the form

$$
D_{0}\left(s, u, Q^{2}\right)=\left(\pi \mu^{-2}\right)^{\frac{D}{2}-2} \frac{\Gamma\left(4-\frac{D}{2}\right) \Gamma^{2}\left(\frac{D}{2}-2\right)}{\Gamma(D-4)} \int_{0}^{1} \int_{0}^{1} \frac{d \xi_{1} d \xi_{2}}{\left(-s \xi_{1}-u \xi_{2}-t \xi_{1} \xi_{2}\right)^{4-\frac{D}{2}}}
$$

Integration over $\xi_{2}$ gives

$$
\begin{aligned}
D_{0}\left(s, u, Q^{2}\right)= & \frac{\left(\pi \mu^{-2}\right)^{\frac{D}{2}-2}}{u} \frac{\Gamma\left(3-\frac{D}{2}\right) \Gamma^{2}\left(\frac{D}{2}-2\right)}{\Gamma(D-4)} \int_{0}^{1} d \xi_{1}\left(1+\frac{t}{u} \xi_{1}\right)^{-1} \\
& \times\left[(-u)^{\frac{D}{2}-3}\left(1+\frac{s+t}{u} \xi_{1}\right)^{\frac{D}{2}-3}-(-s)^{\frac{D}{2}-3} \xi_{1}^{\frac{D}{2}-3}\right] .
\end{aligned}
$$

Next step is to identify the box integral as a combination of Gauss hypergeometric functions ${ }_{2} F_{1}$. We can proceed in two equivalent ways.

First, using $\alpha$ parametrization for the first term in eq. (B.4)

$$
\frac{1}{A B^{n}}=n \int_{0}^{1} d \alpha \frac{\alpha^{n-1}}{[A+(B-A) \alpha]^{n+1}}
$$


and integrating over $\xi_{1}$ we write down the integral as combination of three terms

$$
\begin{aligned}
D_{0}\left(s, u, Q^{2}\right)= & \left(\pi \mu^{-2}\right)^{\frac{D}{2}-2} \frac{\Gamma\left(3-\frac{D}{2}\right) \Gamma^{2}\left(\frac{D}{2}-2\right)}{\Gamma(D-4)} \int_{0}^{1} d \alpha \\
& \times\left[\frac{1}{t}\left(-\frac{(s+t) u}{t}\right)^{\frac{D}{2}-3} \alpha^{2-\frac{D}{2}}\left(1-\alpha \frac{s}{s+t}\right)^{\frac{D}{2}-3}\right. \\
& -\frac{1}{t}\left(-\frac{(s+t)(u+t)}{t}\right)^{\frac{D}{2}-3} \alpha^{2-\frac{D}{2}}\left(1-\alpha \frac{s u}{(s+t)(u+t)}\right)^{\frac{D}{2}-3} \\
& \left.+\frac{(-s)^{\frac{D}{2}-2}}{s u} \alpha^{\frac{D}{2}-3}\left(1+\alpha \frac{t}{u}\right)^{-1}\right] .
\end{aligned}
$$

Next using integral representation for the Gauss hypergeometric function

$$
{ }_{2} F_{1}(a, b, c, z)=\frac{\Gamma(c)}{\Gamma(b) \Gamma(c-b)} \int_{0}^{1} d \alpha \alpha^{b-1}(1-\alpha)^{c-b-1}(1-z \alpha)^{-a},
$$

we get

$$
\begin{aligned}
D_{0}\left(s, u, Q^{2}\right)= & -\left(\pi \mu^{-2}\right)^{\frac{D}{2}-2} \frac{\Gamma\left(2-\frac{D}{2}\right) \Gamma^{2}\left(\frac{D}{2}-2\right)}{\Gamma(D-4)}\left[\frac{(-s)^{\frac{D}{2}-2}}{s u}{ }_{2} F_{1}\left(1, \frac{D}{2}-2, \frac{D}{2}-1,-\frac{t}{u}\right)\right. \\
& -\frac{2-\frac{D}{2}}{3-\frac{D}{2}} \frac{1}{t}\left(-\frac{(s+t) u}{t}\right)^{\frac{D}{2}-3}{ }_{2} F_{1}\left(3-\frac{D}{2}, 3-\frac{D}{2}, 4-\frac{D}{2}, \frac{s}{s+t}\right) \\
& \left.+\frac{2-\frac{D}{2}}{3-\frac{D}{2}} \frac{1}{t}\left(-\frac{(s+t)(u+t)}{t}\right)^{\frac{D}{2}-3}{ }_{2} F_{1}\left(3-\frac{D}{2}, 3-\frac{D}{2}, 4-\frac{D}{2}, \frac{s u}{(s+t)(u+t)}\right)\right] .
\end{aligned}
$$

Further applying the identity

$$
{ }_{2} F_{1}(a, b, c, z)=(1-z)_{2}^{-a} F_{1}\left(c-a, b, c, \frac{z}{z-1}\right),
$$

for the second and third hypergeometric function we get:

$$
\begin{aligned}
D_{0}\left(s, u, Q^{2}\right)= & -\left(\pi \mu^{-2}\right)^{\frac{D}{2}-2} \frac{\Gamma\left(2-\frac{D}{2}\right) \Gamma^{2}\left(\frac{D}{2}-2\right)}{\Gamma(D-4)}\left[\frac{(-s)^{\frac{D}{2}-2}}{s u}{ }_{2} F_{1}\left(1, \frac{D}{2}-2, \frac{D}{2}-1,-\frac{t}{u}\right)\right. \\
& -\frac{2-\frac{D}{2}}{3-\frac{D}{2}} \frac{(-u)^{\frac{D}{2}-3}}{t}{ }_{2} F_{1}\left(1,3-\frac{D}{2}, 4-\frac{D}{2},-\frac{s}{t}\right) \\
& \left.+\frac{2-\frac{D}{2}}{3-\frac{D}{2}} \frac{(-s-t-u)^{\frac{D}{2}-3}}{t}{ }_{2} F_{1}\left(1,3-\frac{D}{2}, 4-\frac{D}{2},-\frac{s u}{(s+t)(u+t)}\right)\right] .
\end{aligned}
$$

Note this representation can also be obtained from eq. (B.4) identifying there the first term with the Appell function using integral representation

$$
F_{1}(a, b, c, d, m, n)=\frac{\Gamma(d)}{\Gamma(a) \Gamma(d-a)} \int_{0}^{1} d \alpha \alpha^{a-1}(1-\alpha)^{d-a-1}(1-m \alpha)^{-b}(1-n \alpha)^{-c},
$$


and further using the identity transforming the Appell function into a combination of two Gauss functions (see proof in the end of this section):

$$
\begin{aligned}
F_{1}(1, b, c, 2, m, n)= & \frac{1}{(1-b-c) m}\left[{ }_{2} F_{1}\left(1, c, b+c, 1-\frac{n}{m}\right)\right. \\
& \left.-(1-m)^{1-b}(1-n)^{-c}{ }_{2} F_{1}\left(1, c, b+c, \frac{m-n}{m(1-n)}\right)\right] .
\end{aligned}
$$

Now we are in the position to derive the final expression for the box integral applying the analytic continuation formula for the Gauss functions in the second and third terms of eq. (B.10):

$$
\begin{aligned}
{ }_{2} F_{1}(a, b, c, z)= & \frac{\Gamma(c) \Gamma(b-a)}{\Gamma(b) \Gamma(c-a)}(-z)_{2}^{-a}{ }_{2} F_{1}\left(a, 1-c+a, 1-b+a, \frac{1}{z}\right) \\
& +\frac{\Gamma(c) \Gamma(a-b)}{\Gamma(a) \Gamma(c-b)}(-z)^{-b}{ }_{2} F_{1}\left(b, 1-c+b, 1-a+b, \frac{1}{z}\right) .
\end{aligned}
$$

Note that only the first term in the identity (B.13) contributes to the expression for the scalar box integral, while the second term vanishes since it generates the equal and opposite in sign contributions in the transformations of the second and third term in eq. (B.10).

Final expression for the box integral is (see also ref. [20]);

$$
\begin{aligned}
D_{0}\left(s, u, Q^{2}\right)= & -\frac{1}{s u}\left[\pi^{\frac{D}{2}-2} \frac{\Gamma\left(2-\frac{D}{2}\right) \Gamma^{2}\left(\frac{D}{2}-2\right)}{\Gamma(D-4)}\right] \\
& \times\left[\left(-\frac{s}{\mu^{2}}\right)^{\frac{D}{2}-2}{ }_{2} F_{1}\left(1, \frac{D}{2}-2, \frac{D}{2}-1,-\frac{t}{u}\right)\right. \\
& +\left(-\frac{u}{\mu^{2}}\right)^{\frac{D}{2}-2}{ }_{2} F_{1}\left(1, \frac{D}{2}-2, \frac{D}{2}-1,-\frac{t}{s}\right) \\
& \left.-\left(-\frac{Q^{2}}{\mu^{2}}\right)_{2}^{\frac{D}{2}-2} F_{1}\left(1, \frac{D}{2}-2, \frac{D}{2}-1,-\frac{Q^{2} t}{s u}\right)\right] .
\end{aligned}
$$

Note that in the on-shell limit $Q^{2}=s+t+u=0$ we reproduce the result for the scalar box given in ref. [24].

The formula for the scalar box integral can be nicely written in terms of the scalar bubble PV function $B_{0}\left(p^{2}\right)(2.7)$ as:

$$
\begin{aligned}
D_{0}\left(s, u, Q^{2}\right)= & -\frac{4}{s u} \frac{D-3}{D-4} I_{0}\left(s, u, Q^{2}\right), \\
I_{0}\left(s, u, Q^{2}\right)= & B_{0}(s){ }_{2} F_{1}\left(1, \frac{D}{2}-2, \frac{D}{2}-1,-\frac{t}{u}\right)+B_{0}(u){ }_{2} F_{1}\left(1, \frac{D}{2}-2, \frac{D}{2}-1,-\frac{t}{s}\right) \\
& -B_{0}\left(Q^{2}\right){ }_{2} F_{1}\left(1, \frac{D}{2}-2, \frac{D}{2}-1,-\frac{Q^{2} t}{s u}\right) .
\end{aligned}
$$


Now we make the $\varepsilon$ expansion of the scalar box diagram. The $\varepsilon$ expansion of the Gauss function reads:

$$
\begin{aligned}
{ }_{2} F_{1}(1,-\varepsilon, 1-\varepsilon, z) & =\frac{1}{1-z}-\sum_{n=0}^{\infty} \varepsilon^{n} \operatorname{Li}_{n}(z) \\
& =1-\sum_{n=1}^{\infty} \varepsilon^{n} \operatorname{Li}_{n}(z)=1+\varepsilon \log (1-z)-\sum_{n=2}^{\infty} \varepsilon^{n} \operatorname{Li}_{n}(z)
\end{aligned}
$$

The proof of eq. (B.16) is given below in this section. Note that the $\mathcal{O}(\varepsilon)$ term in the expansion of the Gauss function does not contribute to the $\varepsilon$ expansion of scalar box due to compensation between the three Gauss functions in eq. (B.15)

$$
\begin{aligned}
& \log \left(1+\frac{t}{s}\right)+\log \left(1+\frac{t}{u}\right)-\log \left(1+\frac{Q^{2} t}{s u}\right) \\
& =\log \left(\frac{s+t}{s}\right)+\log \left(\frac{u+t}{u}\right)-\log \left(\frac{(s+t)(u+t)}{s u}\right) \equiv 0 .
\end{aligned}
$$

Therefore, the $\varepsilon^{2}$ contribution of the Gauss functions reads:

$$
\begin{aligned}
\beta\left(s, u, Q^{2}\right)= & \operatorname{Li}_{2}\left(-\frac{Q^{2} t}{s u}\right)-\operatorname{Li}_{2}\left(-\frac{t}{s}\right)-\operatorname{Li}_{2}\left(-\frac{t}{u}\right) \\
& -\log \left(\frac{-s}{\mu^{2}}\right) \log \left(\frac{u+t}{u}\right)-\log \left(\frac{-u}{\mu^{2}}\right) \log \left(\frac{s+t}{s}\right) \\
& +\log \left(\frac{-Q^{2}}{\mu^{2}}\right) \log \left(\frac{(s+t)(u+t)}{s u}\right) .
\end{aligned}
$$

The expression for $\beta\left(s, u, Q^{2}\right)$ can be simplified using the reflection identity for the dilogarithm:

$$
\mathrm{Li}_{2}(z)+\mathrm{Li}_{2}(1-z)=\frac{\pi^{2}}{6}-\log (z) \log (1-z)
$$

One gets [20]:

$$
\beta\left(s, u, Q^{2}\right)=\operatorname{Li}_{2}\left(1+\frac{t}{s}\right)+\operatorname{Li}_{2}\left(1+\frac{t}{u}\right)-\operatorname{Li}_{2}\left(1+\frac{Q^{2} t}{s u}\right)-\frac{\pi^{2}}{6} .
$$

Next using well-known identity (Abel formula) relating 5 dilogarithms [22, 35]

$$
\begin{aligned}
\frac{1}{2} \log ^{2} \frac{s}{u} & +\operatorname{Li}_{2}\left(1+\frac{t}{s}\right)+\operatorname{Li}_{2}\left(1+\frac{t}{u}\right) \\
& -\operatorname{Li}_{2}\left(1+\frac{Q^{2} t}{s u}\right)+\operatorname{Li}_{2}\left(1-\frac{Q^{2}}{s}\right)+\operatorname{Li}_{2}\left(1-\frac{Q^{2}}{u}\right)=0
\end{aligned}
$$

one can represent the $\beta\left(s, u, Q^{2}\right)$ in terms of two dilogarithms [35]:

$$
\beta\left(s, u, Q^{2}\right)=-\operatorname{Li}_{2}\left(1-\frac{Q^{2}}{s}\right)-\operatorname{Li}_{2}\left(1-\frac{Q^{2}}{u}\right)-\frac{1}{2} \log ^{2} \frac{s}{u}-\frac{\pi^{2}}{6} .
$$

Here for convenience we do not expand the $B_{0}$ function and expand only the Gauss hypergeometric functions. 
Now we derive the $\varepsilon$ expansion of the Gauss hypergeometric function ${ }_{2} F_{1}(1,-\epsilon, 1-\epsilon, z)$ stated in eq. (B.16). We start with the Euler integral representation for this function:

$$
\begin{aligned}
{ }_{2} F_{1}(1,-\varepsilon, 1-\varepsilon, x) & =\frac{\Gamma(1-\varepsilon)}{\Gamma(-\varepsilon)} \int_{0}^{1} \mathrm{~d} t t^{-1-\varepsilon}(1-x t)^{-1} \\
& =-\varepsilon \int_{0}^{1} \mathrm{~d} t \frac{t^{-1-\varepsilon}}{1-x t} .
\end{aligned}
$$

To get rid of the $\varepsilon$-pole in the above integral we perform a partial integration. ${ }^{3}$ Afterwards, we can safely expand the integrand in the $\varepsilon$ series:

$$
\begin{aligned}
{ }_{2} F_{1}(1,-\varepsilon, 1-\varepsilon, x) & =\left.\frac{t^{-\varepsilon}}{1-x t}\right|_{t=0} ^{1}-x \int_{0}^{1} \mathrm{~d} t \frac{t^{-\varepsilon}}{(1-x t)^{2}} \\
& =\frac{1}{1-x}-x \sum_{n=0}^{\infty} \frac{(-\varepsilon)^{n}}{n !} \int_{0}^{1} \mathrm{~d} t \frac{\log ^{n} t}{(1-x t)^{2}} .
\end{aligned}
$$

Now we use the identity

$$
x \frac{(-1)^{n}}{n !} \int_{0}^{1} \mathrm{~d} t \frac{\log ^{n} t}{(1-x t)^{2}}=\operatorname{Li}_{n}(x)
$$

which can be established by induction for all $n \geq 0$ utilizing the recursive definition of the polylogarithms via

$$
\operatorname{Li}_{n+1}(x)=\int_{0}^{x} \frac{\mathrm{d} t}{t} \operatorname{Li}_{n}(t) .
$$

Plugging (B.25) into (B.24) gives

$$
{ }_{2} F_{1}(1,-\varepsilon, 1-\varepsilon, x)=\frac{1}{1-x}-\sum_{n=0}^{\infty} \varepsilon^{n} \operatorname{Li}_{n}(x) .
$$

With $\operatorname{Li}_{0}(x)=\frac{x}{1-x}$ and $\operatorname{Li}_{1}(x)=-\log (1-x)$ we arrive at the all order expansion

$$
{ }_{2} F_{1}(1,-\varepsilon, 1-\varepsilon, x)=1+\varepsilon \log (1-x)-\sum_{n=2}^{\infty} \varepsilon^{n} \operatorname{Li}_{n}(x) .
$$

The gamma functions in eq. (B.14) generates a $\frac{1}{\varepsilon^{2}}$-pole, as

$$
\frac{\Gamma(\varepsilon) \Gamma^{2}(-\varepsilon)}{\Gamma(-2 \varepsilon)}=-\mathrm{e}^{-\gamma_{\mathrm{E}} \varepsilon}\left(\frac{2}{\varepsilon^{2}}-\frac{\pi^{2}}{6}+\mathcal{O}(\varepsilon)\right)
$$

To conclude this section, we prove the identity (B.12), which to our knowledge occurs for the first time in literature. Our proof is based on the use of the Mellin-Barnes integral technique.

We start with the integral representation

$$
F_{1}\left(1, b_{1}, b_{2}, 2, x, y\right)=\int_{0}^{1} \mathrm{~d} t(1-x t)^{-b_{1}}(1-y t)^{-b_{2}} .
$$

\footnotetext{
${ }^{3}$ Subtracting the pole like in section 3.4 would work equally well.
} 
We factorize both multipliers in the integrand applying the Binomi-Mellin-Newton integral

$$
(a+b)^{w}=\frac{1}{\Gamma(-w)} \int_{-i \infty}^{i \infty} \frac{\mathrm{d} z}{2 \pi i} a^{w-z} b^{z} \Gamma(-z) \Gamma(-w+z) .
$$

It yields

$$
\begin{aligned}
F_{1}\left(1, b_{1}, b_{2}, 2, x, y\right)=\frac{1}{\Gamma\left(b_{1}\right) \Gamma\left(b_{2}\right)} & \int_{-i \infty}^{i \infty} \frac{\mathrm{d} z_{1}}{2 \pi i} \int_{-i \infty}^{i \infty} \frac{\mathrm{d} z_{2}}{2 \pi i}(-x)^{z_{1}}(-y)^{z_{2}} \\
& \times \Gamma\left(-z_{1}\right) \Gamma\left(b_{1}+z_{1}\right) \Gamma\left(-z_{2}\right) \Gamma\left(b_{2}+z_{2}\right) \int_{0}^{1} \mathrm{~d} t t^{z_{1}+z_{2}} .
\end{aligned}
$$

Evaluating the integral over $t$ and substituting $z_{1}$ by $z=z_{1}+z_{2}$ lead to

$$
\begin{aligned}
F_{1}\left(1, b_{1}, b_{2}, 2, x, y\right)=\frac{1}{\Gamma\left(b_{1}\right) \Gamma\left(b_{2}\right)} & \int_{-i \infty}^{i \infty} \frac{\mathrm{d} z}{2 \pi i} \frac{(-x)^{z}}{z+1} \int_{-i \infty}^{i \infty} \frac{\mathrm{d} z_{2}}{2 \pi i}\left(-\frac{y}{x}\right)^{z_{2}} \\
& \times \Gamma\left(-z+z_{2}\right) \Gamma\left(b_{2}+z_{2}\right) \Gamma\left(b_{1}+z-z_{2}\right) \Gamma\left(-z_{2}\right) .
\end{aligned}
$$

The integral over the $z_{2}$ variable now matches the Mellin-Barnes representation of a hypergeometric function. It can be subsequently expressed through its real integral representation to obtain

$$
\begin{aligned}
F_{1}\left(1, b_{1}, b_{2}, 2, x, y\right)= & \frac{1}{\Gamma\left(b_{1}\right) \Gamma\left(b_{2}\right)} \int_{-i \infty}^{i \infty} \frac{\mathrm{d} z}{2 \pi i} \frac{(-x)^{z}}{z+1} \frac{\Gamma(-z) \Gamma\left(b_{2}\right) \Gamma\left(b_{1}\right) \Gamma\left(b_{1}+b_{2}+z\right)}{\Gamma\left(b_{1}+b_{2}\right)} \\
& \times{ }_{2} F_{1}\left(-z, b_{2}, b_{1}+b_{2}, 1-\frac{y}{x}\right) \\
= & \frac{1}{\Gamma\left(b_{1}+b_{2}\right)} \int_{-i \infty}^{i \infty} \frac{\mathrm{d} z}{2 \pi i} \frac{(-x)^{z}}{z+1} \Gamma(-z) \Gamma\left(b_{1}+b_{2}+z\right) \\
& \times \frac{\Gamma\left(b_{1}+b_{2}\right)}{\Gamma\left(b_{1}\right) \Gamma\left(b_{2}\right)} \int_{0}^{1} \mathrm{~d} t t^{b_{2}-1}(1-t)^{b_{1}-1}\left(1-t\left(1-\frac{y}{x}\right)\right)^{z} .
\end{aligned}
$$

Interchanging the order of integration and rearranging the terms leads to

$$
\begin{aligned}
F_{1}\left(1, b_{1}, b_{2}, 2, x, y\right)= & \frac{1}{\Gamma\left(b_{1}\right) \Gamma\left(b_{2}\right)} \int_{0}^{1} \mathrm{~d} t t^{b_{2}-1}(1-t)^{b_{1}-1} \\
& \times \int_{-i \infty}^{i \infty} \frac{\mathrm{d} z}{2 \pi i} \frac{\Gamma(z+1) \Gamma\left(b_{1}+b_{2}+z\right) \Gamma(-z)}{\Gamma(z+2)}(-(1-t) x-t y)^{z} .
\end{aligned}
$$

We recognize in the latter formula the $z$ integral as another Mellin-Barnes representation of the Gauss function ${ }_{2} F_{1}$, thus

$$
F_{1}\left(1, b_{1}, b_{2}, 2, x, y\right)=\frac{\Gamma\left(b_{1}+b_{2}\right)}{\Gamma\left(b_{1}\right) \Gamma\left(b_{2}\right)} \int_{0}^{1} \mathrm{~d} t t^{b_{2}-1}(1-t)^{b_{1}-1}{ }_{2} F_{1}\left(1, b_{1}+b_{2}, 2,(1-t) x+t y\right) .
$$

The Gauss function occurring in eq. (B.36) can be further simplified using symmetry under interchange of the first and second argument:

$$
{ }_{2} F_{1}(1, b, 2, x)={ }_{2} F_{1}(b, 1,2, x)=\int_{0}^{1} \mathrm{~d} t(1-x t)^{-b}=\frac{1-(1-x)^{1-b}}{(1-b) x} .
$$


Therefore

$$
\begin{aligned}
F_{1}\left(1, b_{1}, b_{2}, 2, x, y\right)= & \frac{\Gamma\left(b_{1}+b_{2}\right)}{\Gamma\left(b_{1}\right) \Gamma\left(b_{2}\right)} \int_{0}^{1} \mathrm{~d} t t^{b_{2}-1}(1-t)^{b_{1}-1} \frac{1-(1-(1-t) x-t y))^{1-b_{1}-b_{2}}}{\left(1-b_{1}-b_{2}\right)[(1-t) x+t y]} \\
= & \frac{\Gamma\left(b_{1}+b_{2}\right)}{\left(1-b_{1}-b_{2}\right) \Gamma\left(b_{1}\right) \Gamma\left(b_{2}\right)}\left[\frac{1}{x} \int_{0}^{1} \mathrm{~d} t t^{b_{2}-1}(1-t)^{b_{1}-1}\left(1-\frac{x-y}{x} t\right)^{-1}\right. \\
& -\frac{(1-x)^{1-b_{1}-b_{2}}}{x} \int_{0}^{1} \mathrm{~d} t t^{b_{2}-1}(1-t)^{b_{1}-1}\left(1-\frac{x-y}{x} t\right)^{-1} \\
& \left.\times\left(1+\frac{x-y}{1-x} t\right)^{1-b_{1}-b_{2}}\right]=\frac{1}{\left(1-b_{1}-b_{2}\right) x}\left[{ }_{2} F_{1}\left(1, b_{2}, b_{1}+b_{2}, \frac{x-y}{x}\right)\right. \\
& \left.-(1-x)^{1-b_{1}-b_{2}} F_{1}\left(b_{2}, 1, b_{1}+b_{2}-1, b_{1}+b_{2}, \frac{x-y}{x}, \frac{y-x}{1-x}\right)\right],
\end{aligned}
$$

where in the last line we recognized again the integral representations of Gauss and Appell hypergeometric functions.

The Appell function in (B.38) matches the reduction formula (see eq. (1) in section 5.10 in ref. [85]);

$$
F_{1}\left(a, b_{1}, b_{2}, b_{1}+b_{2}, x, y\right)=(1-y)^{-a} F_{1}\left(a, b_{1}, b_{1}+b_{2}, \frac{x-y}{1-y}\right) .
$$

Using (B.39) in (B.38) yields our desired result

$$
\begin{aligned}
F_{1}\left(1, b_{1}, b_{2}, 2, x, y\right)= & \frac{1}{\left(1-b_{1}-b_{2}\right) x}\left[{ }_{2} F_{1}\left(1, b_{2}, b_{1}+b_{2}, \frac{x-y}{x}\right)\right. \\
& \left.-(1-x)^{1-b_{1}-b_{2}}\left(1-\frac{y-x}{1-x}\right)^{-b_{2}}{ }_{2} F_{1}\left(b_{2}, 1, b_{1}+b_{2}, \frac{\frac{x-y}{x}-\frac{y-x}{1-x}}{1-\frac{y-x}{1-x}}\right)\right] \\
= & \frac{1}{\left(1-b_{1}-b_{2}\right) x}\left[{ }_{2} F_{1}\left(1, b_{2}, b_{1}+b_{2}, \frac{x-y}{x}\right)\right. \\
& \left.-(1-x)^{1-b_{1}}(1-y)^{-b_{2}}{ }_{2} F_{1}\left(1, b_{2}, b_{1}+b_{2}, \frac{x-y}{x(1-y)}\right)\right],
\end{aligned}
$$

which we used in the main text (compare eq. (B.12)).

\section{B.2 Vector box integrals}

Here we list the scalar functions occurring in the expansion of the vector box integrals in the orthogonal basis $(P, R, T)$. First, we specify fractioning identities needed to reduce tensorial box diagrams:

Set of identities useful for simplifying the $D_{1}\left(s, u, Q^{2}\right)$ integral

$$
\begin{aligned}
& 2 k P=\Delta_{1}-\Delta_{2}, \\
& 2 k R=-2 \Delta_{0}+\Delta_{1}+\Delta_{2}, \\
& 2 k T=\Delta_{1}-\Delta_{5}+u+k P \frac{u+t}{s}+k R \frac{t-u}{s} .
\end{aligned}
$$


Set of identities useful for simplifying the $D_{1}\left(s, t, Q^{2}\right)$ integral

$$
\begin{aligned}
& 2 k P=\Delta_{1}-\Delta_{2}, \\
& 2 k R=-2 \Delta_{0}+\Delta_{1}+\Delta_{2}, \\
& 2 k T=-\Delta_{2}+\Delta_{6}-t+k P \frac{u+t}{s}+k R \frac{t-u}{s} .
\end{aligned}
$$

Set of the identities useful for the simplifying the $D_{1}\left(t, u, Q^{2}\right)$ integral

$$
\begin{aligned}
& 2 k P=\Delta_{0}-\Delta_{2}-\Delta_{3}+\Delta_{5}-u, \\
& 2 k R=-\Delta_{0}+\Delta_{2}-\Delta_{3}+\Delta_{5}-u, \\
& 2 k T=\Delta_{0}-\Delta_{3}+k P \frac{u+t}{s}+k R \frac{t-u}{s} .
\end{aligned}
$$

Here we use $p_{1}^{2}=p_{2}^{2}=k_{1}^{2}=0$.

The scalar functions occurring in the expansion of vector box integrals read

$$
\begin{aligned}
D_{1 P}^{0125}\left(s, u, Q^{2}\right)= & \frac{P_{\mu}}{P^{2}} D_{1}^{\mu ; 0125}\left(p_{1}, p_{2}, k_{1}\right) \\
= & \frac{1}{2 s}\left[C_{0}\left(u, Q^{2}\right)-C_{0}(u)\right] \\
D_{1 R}^{0125}\left(s, u, Q^{2}\right)= & \frac{R_{\mu}}{R^{2}} D_{1}^{\mu ; 0125}\left(p_{1}, p_{2}, k_{1}\right) \\
= & \frac{1}{2 s}\left[-C_{0}\left(u, Q^{2}\right)-C_{0}(u)+2 C_{0}\left(s, Q^{2}\right)\right] \\
D_{1 T}^{0125}\left(s, u, Q^{2}\right)= & \frac{T_{\mu}}{T^{2}} D_{1}^{\mu ; 0125}\left(p_{1}, p_{2}, k_{1}\right) \\
= & \frac{s}{2 u t}\left[C_{0}(s)-C_{0}\left(u, Q^{2}\right)-u D_{0}\left(s, u, Q^{2}\right)\right. \\
& \left.-(u+t) D_{1 P}^{0125}\left(s, u, Q^{2}\right)-(u-t) D_{1 R}^{0125}\left(s, u, Q^{2}\right)\right] \\
= & \frac{1}{2 u t}\left[(t-u) C_{0}\left(s, Q^{2}\right)-\left(Q^{2}-u\right) C_{0}\left(u, Q^{2}\right)\right. \\
& \left.+s C_{0}(s)+u C_{0}(u)-s u D_{0}\left(s, u, Q^{2}\right)\right] . \\
& \left.+(t+u) D_{1 P}^{0125}\left(s, t, Q^{2}\right)+(t-u) D_{1 R}^{0125}\left(s, u, Q^{2}\right)\right] \\
D_{1 P}^{0126}\left(s, t, Q^{2}\right)= & \frac{P_{\mu}}{P^{2}} D_{1}^{\mu ; 0126}\left(p_{1}, p_{2}, k_{1}\right)=\frac{1}{2 s}\left[C_{0}(t)-C_{0}\left(t, Q^{2}\right)\right]=-D_{1 P}^{0125}\left(s, t, Q^{2}\right), \\
D_{1 R}^{0126}\left(s, t, Q^{2}\right)= & \frac{R_{\mu}}{R^{2}} D_{1}^{\mu ; 0126}\left(p_{1}, p_{2}, k_{1}\right)=\frac{1}{2 s}\left[-C_{0}(t)-C_{0}\left(t, Q^{2}\right)+2 C_{0}\left(s, Q^{2}\right)\right] \\
= & +D_{1 R}^{0125}\left(s, t, Q^{2}\right), \\
D_{1 T}^{0126}\left(s, t, Q^{2}\right)= & \frac{T_{\mu}}{T^{2}} D_{1}^{\mu}\left(s, t, Q^{2}\right) \\
= & \frac{s}{2 u t}\left[-C_{0}(s)+C_{0}\left(t, Q^{2}\right)+t D_{0}\left(s, t, Q^{2}\right)\right. \\
&
\end{aligned}
$$




$$
\begin{aligned}
= & -\frac{1}{2 u t}\left[(u-t) C_{0}\left(s, Q^{2}\right)-\left(Q^{2}-t\right) C_{0}\left(t, Q^{2}\right)\right. \\
& \left.+s C_{0}(s)+t C_{0}(t)-s t D_{0}\left(s, t, Q^{2}\right)\right]=-D_{1 T}^{0125}\left(s, t, Q^{2}\right) . \\
D_{1 P}^{0235}\left(t, u, Q^{2}\right)= & \frac{P_{\mu}}{P^{2}} D_{1}^{\mu ; 0235}\left(p_{1}, p_{2}, k_{1}\right) \\
= & \frac{1}{2 s}\left[C_{0}\left(t, Q^{2}\right)-C_{0}\left(u, Q^{2}\right)-C_{0}(u)+C_{0}(t)-u D_{0}\left(t, u, Q^{2}\right)\right], \\
D_{1 R}^{0235}\left(t, u, Q^{2}\right)= & \frac{R_{\mu}}{R^{2}} D_{1}^{\mu ; 0235}\left(p_{1}, p_{2}, k_{1}\right) \\
= & \frac{1}{2 s}\left[C_{0}\left(t, Q^{2}\right)+C_{0}\left(u, Q^{2}\right)-C_{0}(u)-C_{0}(t)+u D_{0}\left(t, u, Q^{2}\right)\right], \\
D_{1 T}^{0235}\left(t, u, Q^{2}\right)= & \frac{T_{\mu}}{T^{2}} D_{1}^{\mu ; 0235}\left(p_{1}, p_{2}, k_{1}\right) \\
= & \frac{s}{2 u t}\left[-C_{0}\left(t, Q^{2}\right)+C_{0}\left(u, Q^{2}\right)-(u+t) D_{1 P}^{0235}\left(t, u, Q^{2}\right)\right. \\
& \left.-(u-t) D_{1 R}^{0235}\left(t, u, Q^{2}\right)\right]=\frac{1}{2 u t}\left[\left(Q^{2}-u\right) C_{0}\left(u, Q^{2}\right)\right. \\
& \left.-\left(Q^{2}-t\right) C_{0}\left(t, Q^{2}\right)+u C_{0}(u)-t C_{0}(t)+u t D_{0}\left(t, u, Q^{2}\right)\right] .
\end{aligned}
$$

Relations:

$$
\left\{\begin{array}{l}
D_{1 P / 1 T}^{0125}\left(s, u, Q^{2}\right)=-D_{1 P / 1 T}^{0126}\left(s, u, Q^{2}\right) \\
D_{1 R}^{0125}\left(s, u, Q^{2}\right)=+D_{1 R}^{0126}\left(s, u, Q^{2}\right) \\
D_{1 P}^{0125 / 0126}\left(s, u, Q^{2}\right)= \pm D_{1 R}^{0125 / 0126}\left(s, u, Q^{2}\right) \mp \frac{C_{0}\left(s, Q^{2}\right)-C_{0}\left(u, Q^{2}\right)}{s} \\
D_{1 P}^{0235}\left(t, u, Q^{2}\right)=-D_{1 R}^{0235}\left(t, u, Q^{2}\right)+\frac{C_{0}\left(t, Q^{2}\right)-C_{0}(u)}{s}
\end{array}\right.
$$

\section{B.3 Tensor box integrals}

Here we list scalar functions occurring in expansion of tensor box integrals.

\section{Integral $D_{2}^{\mu \nu ; 0125}\left(p_{1}, p_{2}, k_{1}\right)$.}

$$
\begin{aligned}
D_{2 P P}^{0125}\left(s, u, Q^{2}\right)= & \frac{P_{\mu} P_{\nu}}{P^{4}} D_{2}^{\mu \nu ; 0125}\left(p_{1}, p_{2}, k_{1}\right)=\frac{1}{2 s}\left[C_{1 P}^{025}\left(s, u, Q^{2}\right)-C_{1 P}^{015}\left(s, u, Q^{2}\right)\right], \\
D_{2 P R}^{0125}\left(s, u, Q^{2}\right)= & \frac{P_{\mu} R_{\nu}}{P^{2} R^{2}} D_{2}^{\mu \nu ; 0125}\left(p_{1}, p_{2}, k_{1}\right)=\frac{1}{2 s}\left[C_{1 R}^{025}\left(s, u, Q^{2}\right)-C_{1 R}^{015}\left(s, u, Q^{2}\right)\right], \\
D_{2 P T}^{0125}\left(s, u, Q^{2}\right)= & \frac{P_{\mu} T_{\nu}}{P^{2} T^{2}} D_{2}^{\mu \nu ; 0125}\left(p_{1}, p_{2}, k_{1}\right)=\frac{1}{2 s}\left[C_{1 T}^{025}\left(u, Q^{2}\right)-C_{1 T}^{015}(u)\right], \\
D_{2 R R}^{0125}\left(s, u, Q^{2}\right)= & \frac{R_{\mu} R_{\nu}}{R^{4}} D_{2}^{\mu \nu ; 0125}\left(p_{1}, p_{2}, k_{1}\right)=\frac{1}{2 s}\left[-C_{1 R}^{025}\left(s, u, Q^{2}\right)-C_{1 R}^{015}\left(s, u, Q^{2}\right)\right. \\
& \left.+2 C_{1 R}^{078}\left(s, u, Q^{2}\right)-C_{0}\left(s, Q^{2}\right)\right]
\end{aligned}
$$




$$
\begin{aligned}
D_{2 R T}^{0125}\left(s, u, Q^{2}\right)= & \frac{R_{\mu} T_{\nu}}{R^{2} T^{2}} D_{2}^{\mu \nu ; 0125}\left(p_{1}, p_{2}, k_{1}\right)=\frac{1}{2 s}\left[-C_{1 T}^{025}\left(u, Q^{2}\right)-C_{1 T}^{015}(u)\right. \\
& \left.+2 C_{1 T}^{078}\left(s, Q^{2}\right)\right], \\
D_{2 T T}^{0125}\left(s, u, Q^{2}\right)= & \frac{T_{\mu} T_{\nu}}{T^{4}} D_{2}^{\mu \nu ; 0125}\left(p_{1}, p_{2}, k_{1}\right)=\frac{s}{2 u t}\left[\frac{u}{s} C_{1 T}^{015}(u)-\frac{s+t}{s} C_{1 T}^{025}\left(u, Q^{2}\right)\right. \\
& \left.-\frac{u-t}{s} C_{1 T}^{078}\left(s, Q^{2}\right)-u D_{1 T}^{0125}\left(s, u, Q^{2}\right)\right], \\
D_{2 g}^{0125}\left(s, u, Q^{2}\right)= & \frac{g_{\mu \nu}^{\perp}}{D-3} D_{2}^{\mu \nu ; 0125}\left(p_{1}, p_{2}, k_{1}\right)=\frac{1}{D-3}\left[C_{0}\left(s, Q^{2}\right)-s D_{2 P P}^{0125}\left(s, u, Q^{2}\right)\right. \\
& \left.+s D_{2 R R}^{0125}\left(s, u, Q^{2}\right)+\frac{u t}{s} D_{2 T T}^{0125}\left(s, u, Q^{2}\right)\right] .
\end{aligned}
$$

\section{Integral $D_{2}^{\mu \nu ; 0126}\left(p_{1}, p_{2}, k_{1}\right)$.}

$$
\begin{aligned}
& D_{2 P P}^{0126}\left(s, t, Q^{2}\right)=\frac{P_{\mu} P_{\nu}}{P^{4}} D_{2}^{\mu \nu ; 0126}\left(p_{1}, p_{2}, k_{1}\right)=\frac{1}{2 s}\left[C_{1 P}^{026}\left(s, t, Q^{2}\right)-C_{1 P}^{016}\left(s, t, Q^{2}\right)\right] \\
& =+D_{2 P P}^{0125}\left(s, t, Q^{2}\right), \\
& D_{2 P R}^{0126}\left(s, t, Q^{2}\right)=\frac{P_{\mu} R_{\nu}}{P^{2} R^{2}} D_{2}^{\mu \nu ; 0126}\left(p_{1}, p_{2}, k_{1}\right)=\frac{1}{2 s}\left[C_{1 R}^{026}\left(s, t, Q^{2}\right)-C_{1 R}^{016}\left(s, t, Q^{2}\right)\right] \\
& =-D_{2 P P}^{0125}\left(s, t, Q^{2}\right), \\
& D_{2 P T}^{0126}\left(s, t, Q^{2}\right)=\frac{P_{\mu} T_{\nu}}{P^{2} T^{2}} D_{2}^{\mu \nu ; 0126}\left(p_{1}, p_{2}, k_{1}\right)=\frac{1}{2 s}\left[C_{1 T}^{026}(t)-C_{1 T}^{016}\left(t, Q^{2}\right)\right] \\
& =+D_{2 P T}^{0125}\left(s, t, Q^{2}\right), \\
& D_{2 R R}^{0126}\left(s, t, Q^{2}\right)=\frac{R_{\mu} R_{\nu}}{R^{4}} D_{2}^{\mu \nu ; 0126}\left(p_{1}, p_{2}, k_{1}\right)=\frac{1}{2 s}\left[-C_{1 R}^{026}\left(s, t, Q^{2}\right)-C_{1 R}^{016}\left(s, t, Q^{2}\right)\right. \\
& \left.+2 C_{1 R}^{079}\left(s, t, Q^{2}\right)-C_{0}\left(s, Q^{2}\right)\right]=+D_{2 R R}^{0125}\left(s, t, Q^{2}\right), \\
& D_{2 R T}^{0126}\left(s, t, Q^{2}\right)=\frac{R_{\mu} T_{\nu}}{R^{2} T^{2}} D_{2}^{\mu \nu ; 0126}\left(p_{1}, p_{2}, k_{1}\right)=\frac{1}{2 s}\left[-C_{1 T}^{026}\left(t, Q^{2}\right)-C_{1 T}^{016}(t)\right. \\
& \left.+2 C_{1 T}^{079}\left(s, Q^{2}\right)\right]=-D_{2 R T}^{0125}\left(s, t, Q^{2}\right), \\
& D_{2 T T}^{0126}\left(s, t, Q^{2}\right)=\frac{T_{\mu} T_{\nu}}{T^{4}} D_{2}^{\mu \nu ; 0126}\left(p_{1}, p_{2}, k_{1}\right)=\frac{s}{2 u t}\left[-\frac{t}{s} C_{1 T}^{026}(t)+\frac{s+u}{s} C_{1 T}^{016}\left(t, Q^{2}\right)\right. \\
& \left.-\frac{u-t}{s} C_{1 T}^{079}\left(s, Q^{2}\right)+t D_{1 T}^{0126}\left(s, t, Q^{2}\right)\right]=+D_{2 T T}^{0125}\left(s, t, Q^{2}\right), \\
& D_{2 g}^{0126}\left(s, t, Q^{2}\right)=\frac{g_{\mu \nu}^{\perp}}{D-3} D_{2}^{\mu \nu ; 0126}\left(p_{1}, p_{2}, k_{1}\right) \\
& =\frac{1}{D-3}\left[C_{0}\left(s, Q^{2}\right)-s D_{2 P P}^{0126}\left(s, t, Q^{2}\right)+s D_{2 R R}^{0126}\left(s, t, Q^{2}\right)\right. \\
& \left.+\frac{u t}{s} D_{2 T T}^{0126}\left(s, t, Q^{2}\right)\right]=+D_{2 g}^{0125}\left(s, t, Q^{2}\right) \text {. }
\end{aligned}
$$




\section{Integral $D_{2}^{\mu \nu ; 0235}\left(p_{1}, p_{2}, k_{1}\right)$.}

$$
\begin{aligned}
& D_{2 P P}^{0235}\left(t, u, Q^{2}\right)=\frac{P_{\mu} P_{\nu}}{P^{4}} D_{2}^{\mu \nu ; 0235}\left(p_{1}, p_{2}, k_{1}\right)=\frac{1}{2 s}\left[\frac{C_{0}\left(t, Q^{2}\right)}{2}-C_{1 P}^{046}\left(s, t, Q^{2}\right)\right. \\
& \left.+C_{1 P}^{023}\left(s, t, Q^{2}\right)-C_{1 P}^{025}\left(s, u, Q^{2}\right)-C_{1 P}^{035}\left(s, u, Q^{2}\right)-u D_{1 P}^{0235}\left(t, u, Q^{2}\right)\right], \\
& D_{2 P R}^{0235}\left(t, u, Q^{2}\right)=\frac{P_{\mu} R_{\nu}}{P^{2} R^{2}} D_{2}^{\mu \nu ; 0235}\left(p_{1}, p_{2}, k_{1}\right)=\frac{1}{2 s}\left[-\frac{C_{0}\left(t, Q^{2}\right)}{2}-C_{1 R}^{046}\left(s, t, Q^{2}\right)\right. \\
& \left.+C_{1 R}^{023}\left(s, t, Q^{2}\right)-C_{1 R}^{025}\left(s, u, Q^{2}\right)-C_{1 R}^{035}\left(s, u, Q^{2}\right)-u D_{1 R}^{0235}\left(t, u, Q^{2}\right)\right], \\
& D_{2 P T}^{0235}\left(t, u, Q^{2}\right)=\frac{P_{\mu} T_{\nu}}{P^{2} T^{2}} D_{2}^{\mu \nu ; 0235}\left(p_{1}, p_{2}, k_{1}\right)=\frac{1}{2 s}\left[-C_{1 T}^{046}\left(t, Q^{2}\right)+C_{1 T}^{023}(t)\right. \\
& \left.-C_{1 T}^{025}\left(u, Q^{2}\right)-C_{1 T}^{035}(u)-u D_{1 T}^{0235}\left(t, u, Q^{2}\right)\right] \text {, } \\
& D_{2 R R}^{0235}\left(t, u, Q^{2}\right)=\frac{R_{\mu} R_{\nu}}{R^{4}} D_{2}^{\mu \nu ; 0235}\left(p_{1}, p_{2}, k_{1}\right)=\frac{1}{2 s}\left[-\frac{C_{0}\left(t, Q^{2}\right)}{2}-C_{1 R}^{046}\left(s, t, Q^{2}\right)\right. \\
& \left.-C_{1 R}^{023}\left(s, t, Q^{2}\right)+C_{1 R}^{025}\left(s, u, Q^{2}\right)-C_{1 R}^{035}\left(s, u, Q^{2}\right)+u D_{1 R}^{0235}\left(t, u, Q^{2}\right)\right], \\
& D_{2 R T}^{0235}\left(t, u, Q^{2}\right)=\frac{R_{\mu} T_{\nu}}{R^{2} T^{2}} D_{2}^{\mu \nu ; 0235}\left(p_{1}, p_{2}, k_{1}\right)=\frac{1}{2 s}\left[-C_{1 T}^{046}\left(t, Q^{2}\right)-C_{1 T}^{023}(t)+C_{1 T}^{025}\left(u, Q^{2}\right)\right. \\
& \left.-C_{1 T}^{035}(u)+u D_{1 T}^{0235}\left(t, u, Q^{2}\right)\right] \\
& D_{2 T T}^{0235}\left(t, u, Q^{2}\right)=\frac{T_{\mu} T_{\nu}}{T^{4}} D_{2}^{\mu \nu ; 0235}\left(p_{1}, p_{2}, k_{1}\right)=\frac{s}{2 u t}\left[\frac{s+u}{s} C_{1 T}^{046}\left(t, Q^{2}\right)+\frac{s+t}{s} C_{1 T}^{025}\left(u, Q^{2}\right)\right. \\
& \left.+\frac{u}{s} C_{1 T}^{035}(u)-\frac{t}{s} C_{1 T}^{023}(t)+\frac{u t}{s} D_{1 T}^{0235}\left(t, u, Q^{2}\right)\right], \\
& D_{2 g}^{0235}\left(t, u, Q^{2}\right)=\frac{g_{\mu \nu}^{\perp}}{D-3} D_{2}^{\mu \nu ; 0235}\left(p_{1}, p_{2}, k_{1}\right)=\frac{1}{D-3}\left[C_{0}^{046}\left(t, Q^{2}\right)-s D_{2 P P}^{0235}\left(t, u, Q^{2}\right)\right. \\
& \left.+s D_{2 R R}^{0235}\left(t, u, Q^{2}\right)+\frac{u t}{s} D_{2 T T}^{0235}\left(t, u, Q^{2}\right)\right] \text {. }
\end{aligned}
$$

Relations:

$$
\begin{cases}D_{i}^{0125}\left(s, u, Q^{2}\right)=+D_{i}^{0126}\left(s, u, Q^{2}\right), & i=2 g, 2 P P, 2 R R, 2 T T, 2 P T \\ D_{i}^{0125}\left(s, u, Q^{2}\right)=-D_{i}^{0126}\left(s, u, Q^{2}\right), & i=2 P R, 2 T R\end{cases}
$$

Functions occurring in the expansion of tensor rank-3 box integrals (2.52)-(2.54) read:

Integral $D_{3}^{\mu \nu \alpha}\left(s, u, Q^{2}\right)$.

$$
\begin{aligned}
& D_{3 P P P}^{0125}\left(s, u, Q^{2}\right)=\frac{P_{\mu} P_{\nu} P_{\alpha}}{P^{6}} D_{3}^{\mu \nu \alpha}\left(s, u, Q^{2}\right)=\frac{1}{2 s}\left[C_{2 P P}^{025}\left(s, u, Q^{2}\right)-C_{2 P P}^{015}\left(s, u, Q^{2}\right)\right], \\
& D_{3 P P R}^{0125}\left(s, u, Q^{2}\right)=\frac{P_{\mu} P_{\nu} R_{\alpha}}{P^{4} R^{2}} D_{3}^{\mu \nu \alpha}\left(s, u, Q^{2}\right)=\frac{1}{2 s}\left[C_{2 P R}^{025}\left(s, u, Q^{2}\right)-C_{2 P R}^{015}\left(s, u, Q^{2}\right)\right], \\
& D_{3 P P T}^{0125}\left(s, u, Q^{2}\right)=\frac{P_{\mu} P_{\nu} T_{\alpha}}{P^{4} T^{2}} D_{3}^{\mu \nu \alpha}\left(s, u, Q^{2}\right)=\frac{1}{2 s}\left[C_{2 P T}^{025}\left(s, u, Q^{2}\right)-C_{2 P T}^{015}\left(s, u, Q^{2}\right)\right],
\end{aligned}
$$




$$
\begin{aligned}
& D_{3 R R P}^{0125}\left(s, u, Q^{2}\right)=\frac{P_{\mu} R_{\nu} R_{\alpha}}{R^{4} P^{2}} D_{3}^{\mu \nu \alpha}\left(s, u, Q^{2}\right)=\frac{1}{2 s}\left[C_{2 R R}^{025}\left(s, u, Q^{2}\right)-C_{2 R R}^{015}\left(s, u, Q^{2}\right)\right], \\
& D_{3 T T P}^{0125}\left(s, u, Q^{2}\right)=\frac{P_{\mu} T_{\nu} T_{\alpha}}{T^{4} P^{2}} D_{3}^{\mu \nu \alpha}\left(s, u, Q^{2}\right)=\frac{1}{2 s}\left[C_{2 T T}^{025}\left(s, u, Q^{2}\right)-C_{2 T T}^{015}\left(s, u, Q^{2}\right)\right], \\
& D_{3 P R T}^{0125}\left(s, u, Q^{2}\right)=\frac{P_{\mu} R_{\nu} T_{\alpha}}{P^{2} R^{2} T^{2}} D_{3}^{\mu \nu \alpha}\left(s, u, Q^{2}\right)=\frac{1}{2 s}\left[C_{2 R T}^{025}\left(s, u, Q^{2}\right)-C_{2 R T}^{015}\left(s, u, Q^{2}\right)\right], \\
& D_{3 R R R}^{0125}\left(s, u, Q^{2}\right)=\frac{R_{\mu} R_{\nu} R_{\alpha}}{R^{6}} D_{3}^{\mu \nu \alpha}\left(s, u, Q^{2}\right) \\
& =\frac{1}{2 s}\left[-C_{2 R R}^{025}\left(s, u, Q^{2}\right)-C_{2 R R}^{015}\left(s, u, Q^{2}\right)+\frac{C_{0}^{078}\left(s, Q^{2}\right)}{2}\right. \\
& \left.-2 C_{1 R}^{078}\left(s, u, Q^{2}\right)+2 C_{2 R R}^{078}\left(s, u, Q^{2}\right)\right], \\
& D_{3 R R T}^{0125}\left(s, u, Q^{2}\right)=\frac{R_{\mu} R_{\nu} T_{\alpha}}{R^{4} T^{2}} D_{3}^{\mu \nu \alpha}\left(s, u, Q^{2}\right) \\
& =\frac{1}{2 s}\left[-C_{2 R T}^{025}\left(s, u, Q^{2}\right)-C_{2 R T}^{015}\left(s, u, Q^{2}\right)\right. \\
& \left.+2 C_{2 R T}^{078}\left(s, u, Q^{2}\right)-C_{1 T}^{078}\left(s, Q^{2}\right)\right], \\
& D_{3 T T R}^{0125}\left(s, u, Q^{2}\right)=\frac{R_{\mu} T_{\nu} T_{\alpha}}{T^{4} R^{2}} D_{3}^{\mu \nu \alpha}\left(s, u, Q^{2}\right) \\
& =\frac{1}{2 s}\left[-C_{2 T T}^{025}\left(s, u, Q^{2}\right)-C_{2 T T}^{015}\left(s, u, Q^{2}\right)+2 C_{2 T T}^{078}\left(s, u, Q^{2}\right)\right], \\
& D_{3 T T T}^{0125}\left(s, u, Q^{2}\right)=\frac{T_{\mu} T_{\nu} T_{\alpha}}{T^{6}} D_{3}^{\mu \nu \alpha}\left(s, u, Q^{2}\right) \\
& =\frac{s}{2 u t}\left[-u D_{2 T T}^{0125}\left(s, u, Q^{2}\right)+C_{2 T T}^{012}(s)+\frac{u}{s} C_{2 T T}^{015}\left(s, u, Q^{2}\right)\right. \\
& \left.-\frac{s+t}{s} C_{2 T T}^{025}\left(s, u, Q^{2}\right)-\frac{u-t}{s} C_{2 T T}^{078}\left(s, u, Q^{2}\right)\right], \\
& D_{3 g P}^{0125}\left(s, u, Q^{2}\right)=\frac{g_{\mu \nu}^{\perp} P_{\alpha}}{(D-3) P^{2}} D_{3}^{\mu \nu \alpha}\left(s, u, Q^{2}\right) \\
& =\frac{1}{D-3}\left[\frac{C_{0}\left(s, Q^{2}\right)}{2}+C_{1 P}^{078}\left(s, u, Q^{2}\right)+s D_{3 P}^{0125}\left(s, u, Q^{2}\right)\right], \\
& D_{3 g R}^{0125}\left(s, u, Q^{2}\right)=\frac{g_{\mu \nu}^{\perp} R_{\alpha}}{(D-3) R^{2}} D_{3}^{\mu \nu \alpha}\left(s, u, Q^{2}\right) \\
& =\frac{1}{D-3}\left[-\frac{C_{0}\left(s, Q^{2}\right)}{2}+C_{1 R}^{078}\left(s, u, Q^{2}\right)+s D_{3 R}^{0125}\left(s, u, Q^{2}\right)\right], \\
& D_{3 g T}^{0125}\left(s, u, Q^{2}\right)=\frac{g_{\mu \nu}^{\perp} T_{\alpha}}{(D-3) T^{2}} D_{3}^{\mu \nu \alpha}\left(s, u, Q^{2}\right) \\
& =\frac{1}{D-3}\left[C_{1 T}^{078}\left(s, Q^{2}\right)+s D_{3 T}^{0125}\left(s, u, Q^{2}\right)\right] .
\end{aligned}
$$


Here and in the following

$$
\begin{aligned}
D_{3 L}^{0 i j k}\left(s, u, Q^{2}\right) & =-D_{3 L P P}^{0 i j k}\left(s, u, Q^{2}\right)+D_{3 L R R}^{0 i j k}\left(s, u, Q^{2}\right)+\frac{u t}{s^{2}} D_{3 L T T}^{0 i j k}\left(s, u, Q^{2}\right), \\
L & =P, R, T .
\end{aligned}
$$

Integral $D_{3}^{\mu \nu \alpha}\left(s, t, Q^{2}\right)$.

$$
\begin{aligned}
D_{3 P P P}^{0126}\left(s, t, Q^{2}\right)= & \frac{P_{\mu} P_{\nu} P_{\alpha}}{P^{6}} D_{3}^{\mu \nu \alpha}\left(s, t, Q^{2}\right)=\frac{1}{2 s}\left[C_{2 P P}^{026}\left(s, t, Q^{2}\right)-C_{2 P P}^{016}\left(s, t, Q^{2}\right)\right], \\
D_{3 P P R}^{0126}\left(s, t, Q^{2}\right)= & \frac{P_{\mu} P_{\nu} R_{\alpha}}{P^{4} R^{2}} D_{3}^{\mu \nu \alpha}\left(s, t, Q^{2}\right)=\frac{1}{2 s}\left[C_{2 P R}^{026}\left(s, t, Q^{2}\right)-C_{2 P R}^{016}\left(s, t, Q^{2}\right)\right], \\
D_{3 P P T}^{0126}\left(s, t, Q^{2}\right)= & \frac{P_{\mu} P_{\nu} T_{\alpha}}{P^{4} T^{2}} D_{3}^{\mu \nu \alpha}\left(s, t, Q^{2}\right)=\frac{1}{2 s}\left[C_{2 P T}^{026}\left(s, t, Q^{2}\right)-C_{2 P T}^{016}\left(s, t, Q^{2}\right)\right], \\
D_{3 R R P}^{0126}\left(s, t, Q^{2}\right)= & \frac{P_{\mu} R_{\nu} R_{\alpha}}{R^{4} P^{2}} D_{3}^{\mu \nu \alpha}\left(s, t, Q^{2}\right)=\frac{1}{2 s}\left[C_{2 R R}^{026}\left(s, t, Q^{2}\right)-C_{2 R R}^{016}\left(s, t, Q^{2}\right)\right], \\
D_{3 T T P}^{0126}\left(s, t, Q^{2}\right)= & \frac{P_{\mu} T_{\nu} T_{\alpha}}{T^{4} P^{2}} D_{3}^{\mu \nu \alpha}\left(s, t, Q^{2}\right)=\frac{1}{2 s}\left[C_{2 T T}^{026}\left(s, t, Q^{2}\right)-C_{2 T T}^{016}\left(s, t, Q^{2}\right)\right], \\
D_{3 P R T}^{0126}\left(s, t, Q^{2}\right)= & \frac{P_{\mu} R_{\nu} T_{\alpha}}{P^{2} R^{2} T^{2}} D_{3}^{\mu \nu \alpha}\left(s, z, Q^{2}\right)=\frac{1}{2 s}\left[C_{2 R T}^{026}\left(s, t, Q^{2}\right)-C_{2 R T}^{016}\left(s, t, Q^{2}\right)\right], \\
D_{3 R R R}^{0126}\left(s, t, Q^{2}\right)= & \frac{R_{\mu} R_{\nu} R_{\alpha}}{R^{6}} D_{3}^{\mu \nu \alpha}\left(s, t, Q^{2}\right)=\frac{1}{2 s}\left[-C_{2 R R}^{026}\left(s, t, Q^{2}\right)-C_{2 R R}^{016}\left(s, t, Q^{2}\right)\right. \\
& \left.+\frac{C_{0}\left(s, Q^{2}\right)}{2}-2 C_{1 R}^{079}\left(s, t, Q^{2}\right)+2 C_{2 R R}^{079}\left(s, t, Q^{2}\right)\right],
\end{aligned}
$$$$
D_{3 R R T}^{0126}\left(s, t, Q^{2}\right)=\frac{R_{\mu} R_{\nu} T_{\alpha}}{R^{4} T^{2}} D_{3}^{\mu \nu \alpha}\left(s, t, Q^{2}\right)
$$$$
=\frac{1}{2 s}\left[-C_{2 R T}^{026}\left(s, t, Q^{2}\right)-C_{2 R T}^{016}\left(s, t, Q^{2}\right)\right.
$$$$
\left.+2 C_{2 R T}^{079}\left(s, t, Q^{2}\right)-C_{1 T}^{079}\left(t, Q^{2}\right)\right],
$$$$
D_{3 T T R}^{0126}\left(s, t, Q^{2}\right)=\frac{R_{\mu} T_{\nu} T_{\alpha}}{T^{4} R^{2}} D_{3}^{\mu \nu \alpha}\left(s, t, Q^{2}\right)
$$$$
=\frac{1}{2 s}\left[-C_{2 T T}^{026}\left(s, t, Q^{2}\right)-C_{2 T T}^{016}\left(s, t, Q^{2}\right)+2 C_{2 T T}^{079}\left(s, t, Q^{2}\right)\right],
$$$$
D_{3 T T T}^{0126}\left(s, t, Q^{2}\right)=\frac{T_{\mu} T_{\nu} T_{\alpha}}{T^{6}} D_{3}^{\mu \nu \alpha}\left(s, t, Q^{2}\right)
$$$$
=\frac{s}{2 u t}\left[t D_{2 T T}^{0126}\left(s, t, Q^{2}\right)-C_{2 T T}^{012}(s)-\frac{t}{s} C_{2 T T}^{026}\left(s, t, Q^{2}\right)\right.
$$$$
\left.+\frac{s+u}{s} C_{2 T T}^{016}\left(s, t, Q^{2}\right)+\frac{t-u}{s} C_{2 T T}^{079}\left(s, t, Q^{2}\right)\right],
$$

$$
\begin{aligned}
D_{3 g P}^{0126}\left(s, t, Q^{2}\right) & =\frac{g_{\mu \nu}^{\perp} P_{\alpha}}{(D-3) P^{2}} D_{3}^{\mu \nu \alpha}\left(s, t, Q^{2}\right) \\
& =\frac{1}{D-3}\left[\frac{C_{0}\left(s, Q^{2}\right)}{2}+C_{1 P}^{079}\left(s, t, Q^{2}\right)+s D_{3 P}^{0126}\left(s, t, Q^{2}\right)\right],
\end{aligned}
$$




$$
\begin{aligned}
D_{3 g R}^{0126}\left(s, t, Q^{2}\right) & =\frac{g_{\mu \nu}^{\perp} R_{\alpha}}{(D-3) R^{2}} D_{3}^{\mu \nu \alpha}\left(s, t, Q^{2}\right) \\
& =\frac{1}{D-3}\left[-\frac{C_{0}\left(s, Q^{2}\right)}{2}+C_{1 R}^{079}\left(s, t, Q^{2}\right)+s D_{3 R}^{0126}\left(s, t, Q^{2}\right)\right] \\
D_{3 g T}^{0126}\left(s, t, Q^{2}\right) & =\frac{g_{\mu \nu}^{\perp} T_{\alpha}}{(D-3) T^{2}} D_{3}^{\mu \nu \alpha}\left(s, t, Q^{2}\right) \\
& =\frac{1}{D-3}\left[C_{1 T}^{079}\left(t, Q^{2}\right)+s D_{3 T}^{0126}\left(s, t, Q^{2}\right)\right]
\end{aligned}
$$

Relations:

$$
\left\{\begin{array}{l}
D_{i}^{0125}\left(s, u, Q^{2}\right)=+D_{i}^{0126}\left(s, u, Q^{2}\right), \\
i=3 g R, 3 P P R, 3 T T R, 3 P R T, 3 R R R \\
D_{i}^{0125}\left(s, u, Q^{2}\right)=-D_{i}^{0126}\left(s, u, Q^{2}\right), \\
i=3 g P, 3 g T, 3 P P P, 3 T T T, 3 P P T, 3 T T P, 3 R R P, 3 R R T
\end{array}\right.
$$

Integral $D_{3}^{\mu \nu \alpha}\left(t, u, Q^{2}\right)$.

$$
\begin{aligned}
D_{3 P P P}^{0235}\left(t, u, Q^{2}\right)= & \frac{P_{\mu} P_{\nu} P_{\alpha}}{P^{6}} D_{3}^{\mu \nu \alpha}\left(t, u, Q^{2}\right) \\
= & \frac{1}{2 s}\left[C_{2 P P}^{023}\left(s, t, Q^{2}\right)-C_{2 P P}^{025}\left(s, u, Q^{2}\right)-C_{2 P P}^{035}\left(s, u, Q^{2}\right)\right. \\
& \left.+\frac{C_{0}\left(t, Q^{2}\right)}{4}-C_{1 P}^{046}\left(s, t, Q^{2}\right)+C_{2 P P}^{046}\left(s, t, Q^{2}\right)-u D_{2 P P}^{0235}\left(u, t, Q^{2}\right)\right], \\
D_{3 P P R}^{0235}\left(t, u, Q^{2}\right)= & \frac{P_{\mu} P_{\nu} R_{\alpha}}{P^{4} R^{2}} D_{3}^{\mu \nu \alpha}\left(t, u, Q^{2}\right) \\
= & \frac{1}{2 s}\left[C_{2 P R}^{023}\left(s, t, Q^{2}\right)-C_{2 P R}^{025}\left(s, u, Q^{2}\right)-C_{2 P R}^{035}\left(s, t, Q^{2}\right)\right. \\
& -\frac{C_{0}\left(t, Q^{2}\right)}{4}+\frac{C_{1 P}^{046}\left(s, t, Q^{2}\right)}{2}-\frac{C_{1 R}^{046}\left(s, t, Q^{2}\right)}{2}+C_{2 P R}^{046}\left(s, t, Q^{2}\right) \\
& \left.-u D_{2 P R}^{0235}\left(t, u, Q^{2}\right)\right], \\
D_{3 P P T}^{0235}\left(t, u, Q^{2}\right)= & \frac{P_{\mu} P_{\nu} T_{\alpha}}{P^{4} T^{2}} D_{3}^{\mu \nu \alpha}\left(t, u, Q^{2}\right) \\
= & \frac{1}{2 s}\left[C_{2 P T}^{023}\left(s, t, Q^{2}\right)-C_{2 P T}^{025}\left(s, u, Q^{2}\right)-C_{2 P T}^{035}\left(s, u, Q^{2}\right)\right. \\
& \left.-\frac{C_{1 T}^{046}\left(s, t, Q^{2}\right)}{2}+C_{2 P T}^{046}\left(s, t, Q^{2}\right)-u D_{2 P T}^{0235}\left(t, u, Q^{2}\right)\right], \\
& \left.+\frac{C_{0}\left(t, Q^{2}\right)}{4}+C_{1 R}^{046}\left(s, t, Q^{2}\right)+C_{2 R R}^{046}\left(s, t, Q^{2}\right)-u D_{2 R R}^{0235}\left(t, u, Q^{2}\right)\right], \\
D_{3 R R P}^{0235}\left(t, u, Q^{2}\right)= & \frac{P_{\mu} R_{\nu} R_{\alpha}}{R^{4} P^{2}} D_{3}^{\mu \nu \alpha}\left(t, u, Q^{2}\right) \\
= & \frac{1}{2 s}\left[C_{2 R R}^{023}\left(s, t, Q^{2}\right)-C_{2 R R}^{025}\left(s, u, Q^{2}\right)-C_{2 R R}^{035}\left(s, u, Q^{2}\right)\right. \\
&
\end{aligned}
$$




$$
\begin{aligned}
& D_{3 T T P}^{0235}\left(t, u, Q^{2}\right)=\frac{P_{\mu} T_{\nu} T_{\alpha}}{T^{4} P^{2}} D_{3}^{\mu \nu \alpha}\left(t, u, Q^{2}\right) \\
& =\frac{1}{2 s}\left[C_{2 T T}^{023}\left(s, t, Q^{2}\right)-C_{2 T T}^{025}\left(s, u, Q^{2}\right)-C_{2 T T}^{035}\left(s, u, Q^{2}\right)\right. \\
& \left.+C_{2 T T}^{046}\left(s, t, Q^{2}\right)-u D_{2 T T}^{0235}\left(t, u, Q^{2}\right)\right], \\
& D_{3 P R T}^{0235}\left(t, u, Q^{2}\right)=\frac{P_{\mu} R_{\nu} T_{\alpha}}{P^{2} R^{2} T^{2}} D_{3}^{\mu \nu \alpha}\left(t, u, Q^{2}\right) \\
& =\frac{1}{2 s}\left[C_{2 R T}^{023}\left(s, t, Q^{2}\right)-C_{2 R T}^{025}\left(s, u, Q^{2}\right)-C_{2 R T}^{035}\left(s, u, Q^{2}\right)\right. \\
& \left.+\frac{C_{1 T}^{046}\left(t, Q^{2}\right)}{2}+C_{2 R T}^{046}\left(s, t, Q^{2}\right)-u D_{2 R T}^{0235}\left(t, u, Q^{2}\right)\right], \\
& D_{3 R R R}^{0235}\left(t, u, Q^{2}\right)=\frac{R_{\mu} R_{\nu} R_{\alpha}}{R^{6}} D_{3}^{\mu \nu \alpha}\left(t, u, Q^{2}\right) \\
& =\frac{1}{2 s}\left[-C_{2 R R}^{023}\left(s, t, Q^{2}\right)+C_{2 R R}^{025}\left(s, u, Q^{2}\right)-C_{2 R R}^{035}\left(s, u, Q^{2}\right)\right. \\
& \left.+\frac{C_{0}^{046}\left(t, Q^{2}\right)}{4}+C_{1 R}^{046}\left(s, t, Q^{2}\right)+C_{2 R R}^{046}\left(s, t, Q^{2}\right)+u D_{2 R R}^{0235}\left(t, u, Q^{2}\right)\right], \\
& D_{3 R R T}^{0235}\left(t, u, Q^{2}\right)=\frac{R_{\mu} R_{\nu} T_{\alpha}}{R^{4} T^{2}} D_{3}^{\mu \nu \alpha}\left(t, u, Q^{2}\right) \\
& =\frac{1}{2 s}\left[-C_{2 R T}^{023}\left(s, t, Q^{2}\right)+C_{2 R T}^{025}\left(s, u, Q^{2}\right)-C_{2 R T}^{035}\left(s, u, Q^{2}\right)\right. \\
& \left.+\frac{C_{1 T}^{046}\left(t, Q^{2}\right)}{2}++C_{2 R T}^{046}\left(s, t, Q^{2}\right)+u D_{2 R T}^{0235}\left(u, t, Q^{2}\right)\right], \\
& D_{3 T T R}^{0235}\left(t, u, Q^{2}\right)=\frac{R_{\mu} T_{\nu} T_{\alpha}}{T^{4} R^{2}} D_{3}^{\mu \nu \alpha}\left(t, u, Q^{2}\right) \\
& =\frac{1}{2 s}\left[-C_{2 T T}^{023}\left(s, t, Q^{2}\right)+C_{2 T T}^{025}\left(s, u, Q^{2}\right)-C_{2 T T}^{035}\left(s, u, Q^{2}\right)\right. \\
& \left.+C_{2 T T}^{046}\left(s, t, Q^{2}\right)+u D_{2 T T}^{0235}\left(u, t, Q^{2}\right)\right], \\
& D_{3 T T T}^{0235}\left(t, u, Q^{2}\right)=\frac{T_{\mu} T_{\nu} T_{\alpha}}{T^{6}} D_{3}^{\mu \nu \alpha}\left(t, u, Q^{2}\right) \\
& =\frac{1}{2 u t}\left[-t C_{2 T T}^{023}\left(s, t, Q^{2}\right)+(s+t) C_{2 T T}^{025}\left(s, u, Q^{2}\right)+u C_{2 T T}^{035}\left(s, u, Q^{2}\right)\right. \\
& \left.-(s+u) C_{2 T T}^{046}\left(s, t, Q^{2}\right)+D_{2 T T}^{0235}\left(u, t, Q^{2}\right)\right], \\
& D_{3 g P}^{0235}\left(t, u, Q^{2}\right)=\frac{g_{\mu \nu}^{\perp} P_{\alpha}}{(D-3) P^{2}} D_{3}^{\mu \nu \alpha}\left(t, u, Q^{2}\right) \\
& =\frac{1}{D-3}\left[\frac{C_{0}\left(t, Q^{2}\right)}{2}-C_{1 P}^{046}\left(s, t, Q^{2}\right)+s D_{3 P}^{0235}\left(u, t, Q^{2}\right)\right],
\end{aligned}
$$




$$
\begin{aligned}
D_{3 g R}^{0235}\left(t, u, Q^{2}\right) & =\frac{g_{\mu \nu}^{\perp} R_{\alpha}}{(D-3) R^{2}} D_{3}^{\mu \nu \alpha}\left(s, t, Q^{2}\right) \\
& =\frac{1}{D-3}\left[-\frac{C_{0}\left(t, Q^{2}\right)}{2}+C_{1 R}^{046}\left(s, t, Q^{2}\right)+s D_{3 R}^{0235}\left(u, t, Q^{2}\right)\right], \\
D_{3 g T}^{0235}\left(t, u, Q^{2}\right) & =\frac{g_{\mu \nu}^{\perp} T_{\alpha}}{(D-3) T^{2}} D_{3}^{\mu \nu \alpha}\left(s, t, Q^{2}\right) \\
& =\frac{1}{D-3}\left[-C_{1 T}^{046}\left(t, Q^{2}\right)+s D_{3 T}^{0235}\left(u, t, Q^{2}\right)\right] .
\end{aligned}
$$

\section{Spherical integration in dimensional regularization}

The key tool to handle PSIs in dimensional regularization is a generalization of spherical integration to arbitrary dimension. In our consideration we follow handbook [86]. For any integer $n$-dimension we can introduce spherical coordinates. For $n=2$ dimension we have

$$
\vec{x}^{(2)}=r_{2} \vec{u}^{(2)}\left(\theta_{1}\right) \quad \text { with } \quad \vec{u}^{(2)}\left(\theta_{1}\right)=\left(\sin \theta_{1}, \cos \theta_{1}\right),
$$

with module of vector $0 \leq r_{2}<\infty$ and angle $0 \leq \theta_{1} \leq 2 \pi$. Here and in the following, we denote the $n$-dimensional length of a vector as $r_{n}$ and $\vec{u}^{(n)}\left(\theta_{1}, \ldots, \theta_{n-1}\right)$ is a unit vector depending on $n-1$ angular coordinates $\theta_{i}$.

Suppose that we know how to express the unit vector $\vec{u}^{(n-1)}\left(\theta_{1}, \ldots, \theta_{n-2}\right)$ in terms of $n-2$ angular variables. Then we can write $\vec{x}^{(n)}$ in cylindrical coordinates

$$
\vec{x}^{(n)}=\left(r_{n-1} \vec{u}^{(n-1)}\left(\theta_{1}, \ldots, \theta_{n-2}\right), x_{n}\right) .
$$

Changing variables to spherical ones by the relations

$$
r_{n-1}=r_{n} \sin \theta_{n-1}, \quad x_{n}=r_{n} \cos \theta_{n-1},
$$

where $0 \leq r_{n} \leq \infty$ and $0 \leq \theta_{n-1}<\pi$ gives

$$
\begin{aligned}
\vec{x}^{(n)} & =r_{n}\left(\sin \theta_{n-1} \vec{u}^{(n-1)}\left(\theta_{1}, \ldots, \theta_{n-2}\right), \cos \theta_{n-1}\right) \\
& =r_{n} \vec{u}^{(n)}\left(\theta_{1}, \ldots, \theta_{n-1}\right) .
\end{aligned}
$$

With this procedure we have constructed spherical coordinates for arbitrary integer dimension $n \geq 2$.

Now we are facing the issue of how to evaluate integrals in $n$ dimensions without specifying $n$. This will enable us to formally extend the resulting formula to non-integer $n$. The central idea to do so is to first examine on which set of angular variables the integrand depends. For our purposes, it suffices to consider scalar integrands where the integration variable $\vec{x}^{(n)}$ only appears in scalar products of the form $\vec{x}^{(n)} \cdot \vec{y}^{(n)}$ with a fixed $n$-dimensional vector of the specific form $\vec{y}^{(n)}=\left(\overrightarrow{0}_{n-2}, y_{2}, y_{1}\right)$ or as an absolute square $\vec{x}^{(n)} \cdot \vec{x}^{(n)}=r_{n}^{2}$. We can expand $\vec{x}^{(n)}$ with our recursion in terms of angular variables, 
obtaining

$$
\begin{aligned}
\vec{x}^{(n)} & =r_{n} \vec{u}^{(n)}\left(\theta_{1}, \ldots, \theta_{n-1}\right) \\
& =r_{n}\left(\sin \theta_{n-1} \vec{u}^{(n-1)}\left(\theta_{1}, \ldots, \theta_{n-2}\right), \cos \theta_{n-1}\right) \\
& =r_{n}\left(\sin \theta_{n-1} \sin \theta_{n-2} \vec{u}^{(n-2)}\left(\theta_{1}, \ldots, \theta_{n-3}\right), \sin \theta_{n-1} \cos \theta_{n-2}, \cos \theta_{n-1}\right) .
\end{aligned}
$$

Therefore,

$$
\vec{x}^{(n)} \cdot \vec{y}^{(n)}=r_{n}\left(y_{2} \sin \theta_{n-1} \cos \theta_{n-2}+y_{1} \cos \theta_{n-1}\right) .
$$

This implies that we can integrate over all angular variables except for $\theta_{n-2}$ and $\theta_{n-1}$ as we have integrands of the form $f\left(r_{n}, \theta_{n-1}, \theta_{n-2}\right)$. The next step is to express the $\int \mathrm{d}^{n} x$ integral in terms of our spherical coordinates with explicit $\theta_{n-2}$ and $\theta_{n-1}$ integrations.

The integral in spherical coordinates in $n$-dimensions has the form

$$
\int \mathrm{d}^{n} x=\int_{0}^{\infty} \mathrm{d} r_{n} r_{n}^{n-1} \int \mathrm{d} \Omega_{n-1} .
$$

Constructing $\int \mathrm{d} \Omega_{n-1}$ iteratively using the spherical coordinates defined above yields

$$
\int \mathrm{d} \Omega_{n-1}=\int_{0}^{2 \pi} \mathrm{d} \theta_{1} \prod_{k=2}^{n-1} \int_{0}^{\pi} \mathrm{d} \theta_{k} \sin ^{k-1} \theta_{k} .
$$

The second part is

$$
\int_{0}^{\pi} \mathrm{d} \theta \sin ^{k} \theta=B\left(\frac{1}{2}, \frac{k+1}{2}\right),
$$

and integrating out all angles yields

$$
\Omega_{n}=\int \mathrm{d} \Omega_{n}=2 \pi \prod_{k=1}^{n-1} B\left(\frac{1}{2}, \frac{k+1}{2}\right)=\frac{2 \pi^{\frac{n+1}{2}}}{\Gamma\left(\frac{n+1}{2}\right)} .
$$

Formula (C.10) allows for continuation to arbitrary complex $n$ (except for poles). This observation takes us a step closer to formally evaluate integrals in non-integer dimensions.

The idea for calculating the integrals of interest $\int \mathrm{d} \Omega_{n}$ with $n$ non-integer is to split it in two parts. One part is the integral over $k$ angles, where $k$ is a fixed integer. In our case this part contains the integrand $f\left(r_{n}, \theta_{n-1}, \theta_{n-2}\right)$ with $k=2$. The remaining part is an $n-k$ dimensional spherically symmetric integral, which can be also evaluated for non-integer $n-k$ using the identity (C.10). The splitting we need is

$$
\begin{aligned}
\int \mathrm{d} \Omega_{n-1} & =\int_{0}^{\pi} \mathrm{d} \theta_{n-1} \sin ^{n-2} \theta_{n-1} \int \mathrm{d} \Omega_{n-2} \\
& =\int_{0}^{\pi} \mathrm{d} \theta_{n-1} \sin ^{n-2} \theta_{n-1} \int_{0}^{\pi} \mathrm{d} \theta_{n-3} \sin ^{n-2} \theta_{n-2} \int \mathrm{d} \Omega_{n-3} .
\end{aligned}
$$

Thus we obtain for our integral

$$
\begin{aligned}
& \int \mathrm{d}^{n} x f\left(r_{n}, \theta_{n-1}, \theta_{n-2}\right)=\int_{0}^{\infty} \mathrm{d} r_{n} r_{n}^{n-1} \int \mathrm{d} \Omega_{n-1} f\left(r_{n}, \theta_{n-1}, \theta_{n-2}\right) \\
& =\Omega_{n-3} \int_{0}^{\infty} \mathrm{d} r_{n} r_{n}^{n-1} \int_{0}^{\pi} \mathrm{d} \theta_{n-1} \sin ^{n-2} \theta_{n-1} \int_{0}^{\pi} \mathrm{d} \theta_{n-2} \sin ^{n-3} \theta_{n-2} f\left(r_{n}, \theta_{n-1}, \theta_{n-2}\right) .
\end{aligned}
$$

Commonly the angles are relabeled $\theta_{n-1} \rightarrow \theta_{1}$ and $\theta_{n-2} \rightarrow \theta_{2}$ at this stage. 


\section{Partial fraction decomposition of linear propagators}

A common phrase to read about PSI is that it involves a tedious partial fraction decomposition. Algorithms of how to perform them are known. However, to the best of the authors knowledge, the partial fraction decomposition was never viewed in a wider context. Especially the question under which circumstances one can reduce the number of angular integrals to be calculated often lacks an explanation. In this section we want to discuss a simple geometric representation of the occurring propagators, which is straightforwardly connected with Minkowski momentum space. We will see that any partial fractioning has its root in just one basic formula (D.7). This formula can then be extended to not only a general picture of partial fraction decompositions, but also to a nice and useful formula for the splitting of double-massive propagators to single-massive ones used in section 3.2.6 to calculate the double massive integral in $D$ dimensions.

To set the starting stage, we consider Euclidean vectors $\mathbf{v}_{\mathbf{i}} \in \mathbb{R}^{D-1}$ and define there scaled linear propagator

$$
\Delta_{\mathbf{k}}\left(\mathbf{v}_{\mathbf{i}}\right)=\frac{1}{1-\mathbf{v}_{\mathbf{i}} \cdot \mathbf{k}}
$$

where $\mathbf{k}$ is the PSI integration momentum choosen for convenience as an arbitrary unit vector. How $\Delta_{\mathbf{k}}\left(\mathbf{v}_{\mathbf{i}}\right)$ is connected with physical propagators will be clarified in section D.5. For convenience in our consideration we introduce the shorten notation for the product of $N$ propagators:

$$
\Delta_{\mathbf{k}}\left(\mathbf{v}_{\mathbf{1}}, \mathbf{v}_{\mathbf{2}}, \cdots, \mathbf{v}_{\mathbf{N}}\right) \equiv \Delta_{\mathbf{k}}\left(\mathbf{v}_{\mathbf{1}}\right) \Delta_{\mathbf{k}}\left(\mathbf{v}_{\mathbf{2}}\right) \cdots \Delta_{\mathbf{k}}\left(\mathbf{v}_{\mathbf{N}}\right) .
$$

\section{D.1 Two-point splitting lemma}

We start by proving the so-called two-point splitting lemma allowing to factorize two variables in two-propagator product $\Delta_{\mathbf{k}}\left(\mathbf{v}_{\mathbf{1}}, \mathbf{v}_{\mathbf{2}}\right)$ [see definition in eq. (D.2)] by introducing two-propagator products of the vectors $v_{1}$ and $v_{2}$ with another (third) arbitrary Euclidean vector $v_{3}$. In particular, we are interested in deriving a decomposition of the form

$$
\Delta_{\mathbf{k}}\left(\mathbf{v}_{\mathbf{1}}, \mathbf{v}_{\mathbf{2}}\right)=\lambda \Delta_{\mathbf{k}}\left(\mathbf{v}_{\mathbf{1}}, \mathbf{v}_{\mathbf{3}}\right)+\mu \Delta_{\mathbf{k}}\left(\mathbf{v}_{\mathbf{2}}, \mathbf{v}_{\mathbf{3}}\right)
$$

where $\lambda$ and $\mu$ are two real parameters. Such identity will be useful for manipulations of products of propagators containing $\mathbf{v}_{\mathbf{1}}$ and $\mathbf{v}_{\mathbf{2}}$ in PSIs. Starting from r.h.s. of eq. (D.3), we arrive at

$$
\begin{aligned}
& \lambda \Delta_{\mathbf{k}}\left(\mathbf{v}_{\mathbf{1}}, \mathbf{v}_{\mathbf{3}}\right)+\mu \Delta_{\mathbf{k}}\left(\mathbf{v}_{\mathbf{2}}, \mathbf{v}_{\mathbf{3}}\right) \\
& =\left(\lambda \Delta_{\mathbf{k}}^{-1}\left(\mathbf{v}_{\mathbf{2}}\right)+\mu \Delta_{\mathbf{k}}^{-1}\left(\mathbf{v}_{\mathbf{1}}\right)\right) \Delta_{\mathbf{k}}\left(\mathbf{v}_{\mathbf{1}}, \mathbf{v}_{\mathbf{2}}, \mathbf{v}_{\mathbf{3}}\right) \\
& =\left[\left(\lambda \Delta_{\mathbf{k}}^{-1}\left(\mathbf{v}_{\mathbf{2}}\right)+\mu \Delta_{\mathbf{k}}^{-1}\left(\mathbf{v}_{\mathbf{1}}\right)\right) \Delta_{\mathbf{k}}\left(\mathbf{v}_{\mathbf{3}}\right)\right] \Delta_{\mathbf{k}}\left(\mathbf{v}_{\mathbf{1}}, \mathbf{v}_{\mathbf{2}}\right) .
\end{aligned}
$$

Comparing the latter with l.h.s.of eq. (D.3) we conclude that the condition

$$
\left(\lambda \Delta_{\mathbf{k}}^{-1}\left(\mathbf{v}_{\mathbf{2}}\right)+\mu \Delta_{\mathbf{k}}^{-1}\left(\mathbf{v}_{\mathbf{1}}\right)\right) \Delta_{\mathbf{k}}\left(\mathbf{v}_{\mathbf{3}}\right) \equiv 1,
$$




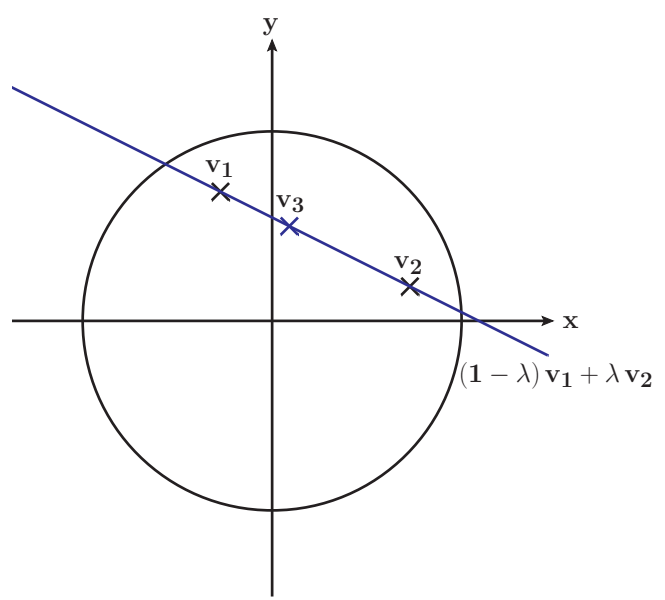

Figure 2. A graphical representation of the two-point splitting lemma. The two-dimensional $(x y)$ plane is spanned by two arbitrary linearly independent vectors $\mathbf{v}_{\mathbf{1}}$ and $\mathbf{v}_{\mathbf{2}}$. The head of the third vector $\mathbf{v}_{\mathbf{3}}$ (we show one typical example) lies in the straight line connecting the heads of $\mathbf{v}_{\mathbf{1}}$ and $\mathbf{v}_{\mathbf{2}}$. The circle represents all vectors with $\mathbf{v} \cdot \mathbf{v}=1$.

must hold. It gives a system of two equations on two arbitrary real parameters $\lambda$ and $\mu$ and vector $\mathbf{v}_{\mathbf{3}}$ :

$$
\left\{\begin{array}{c}
\lambda+\mu=1 \\
\mu \mathbf{v}_{\mathbf{1}}+\lambda \mathbf{v}_{\mathbf{2}}=\mathbf{v}_{\mathbf{3}},
\end{array}\right.
$$

From eq. (D.6) it follows that the parameter $\lambda$ can be chosen arbitrarily, while the second parameter $\mu$ and vector $\mathbf{v}_{\mathbf{3}}$ are then fixed as $\mu=1-\lambda$ and $\mathbf{v}_{\mathbf{3}}=(1-\lambda) \mathbf{v}_{\mathbf{1}}+\lambda \mathbf{v}_{\mathbf{2}}$.

Therefore, we are now in the position to formulate the Two-Point Splitting Lemma:

$$
\Delta_{\mathbf{k}}\left(\mathbf{v}_{\mathbf{1}}, \mathbf{v}_{\mathbf{2}}\right)=\lambda \Delta_{\mathbf{k}}\left(\mathbf{v}_{\mathbf{1}}, \mathbf{v}_{\mathbf{3}}\right)+(1-\lambda) \Delta_{\mathbf{k}}\left(\mathbf{v}_{\mathbf{2}}, \mathbf{v}_{\mathbf{3}}\right),
$$

where $\mathbf{v}_{\mathbf{3}}=(1-\lambda) \mathbf{v}_{\mathbf{1}}+\lambda \mathbf{v}_{\mathbf{2}}$.

Geometrical interpretation of the two-point splitting lemma is that the heads of the vector $\mathbf{v}_{\mathbf{3}}$ defined as $\mathbf{v}_{\mathbf{3}}=(1-\lambda) \mathbf{v}_{\mathbf{1}}+\lambda \mathbf{v}_{\mathbf{2}}$ with $\lambda$ being arbitrary real parameter represents the line passing through the heads of $\mathbf{v}_{\mathbf{1}}$ and $\mathbf{v}_{\mathbf{2}}$. In other words, it is a definition of a line by two linear independent vectors. All three vectors $\mathbf{v}_{\mathbf{1}}, \mathbf{v}_{\mathbf{2}}$, and $\mathbf{v}_{\mathbf{3}}$ obviously lie in the two-dimensional plane spanned by $\mathbf{v}_{\mathbf{1}}$ and $\mathbf{v}_{\mathbf{2}}$. This is depicted in figure 2 .

For $j, l \in \mathbb{N}$ the two-point splitting lemma can be generalized to

$$
\begin{aligned}
\Delta_{\mathbf{k}}^{j}\left(\mathbf{v}_{\mathbf{1}}\right) \Delta_{\mathbf{k}}^{l}\left(\mathbf{v}_{\mathbf{2}}\right)= & \sum_{n=0}^{j-1}\left(\begin{array}{c}
l-1+n \\
l-1
\end{array}\right) \lambda^{l}(1-\lambda)^{n} \Delta_{\mathbf{k}}^{j-n}\left(\mathbf{v}_{\mathbf{1}}\right) \Delta_{\mathbf{k}}^{l+n}\left(\mathbf{v}_{\mathbf{3}}\right) \\
& +\sum_{n=0}^{l-1}\left(\begin{array}{c}
j-1+n \\
j-1
\end{array}\right) \lambda^{n}(1-\lambda)^{j} \Delta_{\mathbf{k}}^{l-n}\left(\mathbf{v}_{\mathbf{2}}\right) \Delta_{\mathbf{k}}^{j+n}\left(\mathbf{v}_{\mathbf{3}}\right) .
\end{aligned}
$$

To prove eq. (D.8) we employ the binomial summation formula

$$
\sum_{n=0}^{N}\left(\begin{array}{c}
j-1+n \\
j-1
\end{array}\right)=\left(\begin{array}{c}
j+N+1 \\
j
\end{array}\right),
$$

and use mathematical induction. 


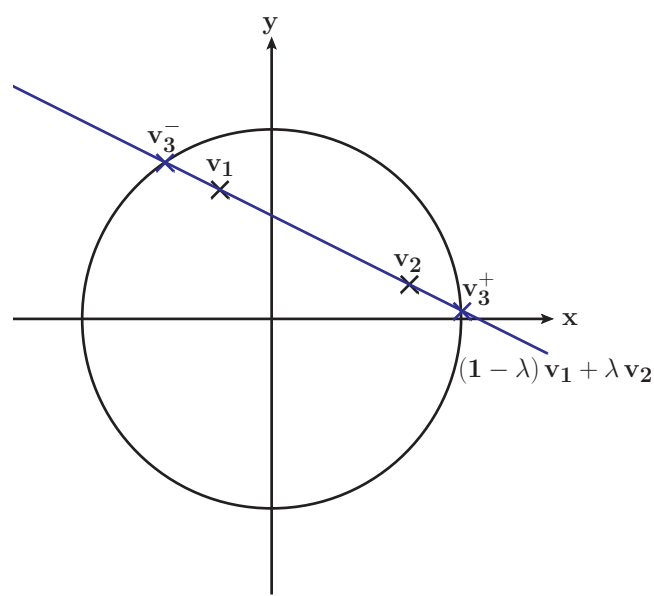

Figure 3. Graphical representation of double-to-single-massive splitting. $\mathbf{v}_{\mathbf{3}}^{ \pm}$is chosen as one of the intersections of the unit circle with the line through $\mathbf{v}_{\mathbf{1}}$ and $\mathbf{v}_{\mathbf{2}}$.

\section{D.2 Two-mass splitting and two-point partial fractioning}

The two-mass splitting now becomes a simple corollary of the two-point splitting lemma. We just have to choose $\mathbf{v}_{\mathbf{3}}$ such that $\mathbf{v}_{\mathbf{3}} \cdot \mathbf{v}_{\mathbf{3}}=1$. To achieve this we have to take $\mathbf{v}_{\mathbf{3}}$ as the intersection of the line $(1-\lambda) \mathbf{v}_{\mathbf{1}}+\lambda \mathbf{v}_{\mathbf{2}}$ with the unit circle. The condition

$$
\left|(1-\lambda) \mathbf{v}_{\mathbf{1}}+\lambda \mathbf{v}_{\mathbf{2}}\right|^{2} \stackrel{!}{=} 1
$$

leads to the quadratic equation (see figure 3 )

$$
\lambda^{2}\left(\mathbf{v}_{\mathbf{2}}-\mathbf{v}_{\mathbf{1}}\right)^{2}+2 \lambda \mathbf{v}_{\mathbf{1}} \cdot\left(\mathbf{v}_{\mathbf{2}}-\mathbf{v}_{\mathbf{1}}\right)+\mathbf{v}_{\mathbf{1}} \cdot \mathbf{v}_{\mathbf{1}}-1 \stackrel{!}{=} 0 .
$$

Defining the scaled Mandelstam variables

$$
v_{11}=1-\mathbf{v}_{\mathbf{1}} \cdot \mathbf{v}_{\mathbf{1}}, \quad v_{12}=1-\mathbf{v}_{\mathbf{1}} \cdot \mathbf{v}_{\mathbf{2}}, \quad v_{22}=1-\mathbf{v}_{\mathbf{2}} \cdot \mathbf{v}_{\mathbf{2}},
$$

we write down eq. (D.11) as

$$
\lambda^{2}\left(2 v_{12}-v_{11}-v_{22}\right)+2 \lambda\left(v_{11}-v_{12}\right)-v_{11}=0 .
$$

The solution of the latter equation is

$$
\lambda_{ \pm}=\frac{v_{12}-v_{11} \pm \sqrt{v_{12}^{2}-v_{11} v_{22}}}{2 v_{12}-v_{11}-v_{22}} .
$$

The massless vectors associated with $\mathbf{v}_{\mathbf{1}}$ and $\mathbf{v}_{\mathbf{2}}$ thus are

$$
\mathbf{v}_{\mathbf{3}}^{ \pm}=\frac{v_{12}-v_{22} \pm \sqrt{v_{12}^{2}-v_{11} v_{22}}}{2 v_{12}-v_{11}-v_{22}} \mathbf{v}_{\mathbf{1}}+\frac{v_{12}-v_{11} \mp \sqrt{v_{12}^{2}-v_{11} v_{22}}}{2 v_{12}-v_{11}-v_{22}} \mathbf{v}_{\mathbf{2}} .
$$

Using the $\lambda$ from eq. (D.14) and the associated vector from (D.15) we have the Double-toSingle-Massive Splitting (or simply Two-mass splitting)

$$
\Delta_{\mathbf{k}}\left(\mathbf{v}_{\mathbf{1}}, \mathbf{v}_{\mathbf{2}}\right)=\lambda_{ \pm} \Delta_{\mathbf{k}}\left(\mathbf{v}_{\mathbf{1}}, \mathbf{v}_{\mathbf{3}}^{ \pm}\right)+\left(1-\lambda_{ \pm}\right) \Delta_{\mathbf{k}}\left(\mathbf{v}_{\mathbf{2}}, \mathbf{v}_{\mathbf{3}}^{ \pm}\right) .
$$




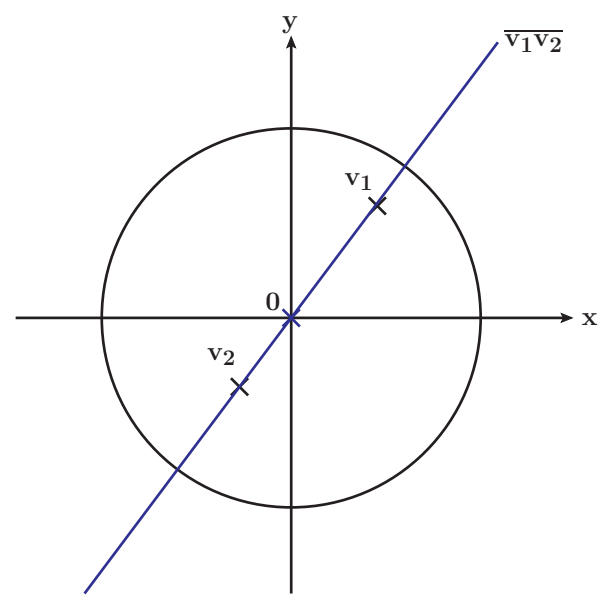

Figure 4. Graphical illustration of two-point partial fractioning. The origin lies on the line $\overline{\mathbf{V}_{\mathbf{1}} \mathbf{V}_{\mathbf{2}}}$, we can use $\Delta_{\mathbf{k}}(\mathbf{0})$ to split $\Delta_{\mathbf{k}}\left(\mathbf{v}_{\mathbf{1}}\right)$ and $\Delta_{\mathbf{k}}\left(\mathbf{v}_{\mathbf{2}}\right)$.

It splits the double-massive propagator on the left into two single-massive propagators on the right. This proves very useful for the calculation of the double-massive integral with integer coefficients.

The two mass-splitting becomes even more powerful in combination with the generalized form of the two point splitting lemma. For $j, l \in \mathbb{N}$ it holds

$$
\begin{aligned}
\Delta_{\mathbf{k}}^{j}\left(\mathbf{v}_{\mathbf{1}}\right) \Delta_{\mathbf{k}}^{l}\left(\mathbf{v}_{\mathbf{2}}\right)= & \sum_{n=0}^{j-1}\left(\begin{array}{c}
l-1+n \\
l-1
\end{array}\right) \lambda_{ \pm}^{l}\left(1-\lambda_{ \pm}\right)^{n} \Delta_{\mathbf{k}}^{j-n}\left(\mathbf{v}_{\mathbf{1}}\right) \Delta_{\mathbf{k}}^{l+n}\left(\mathbf{v}_{\mathbf{3}}^{ \pm}\right) \\
& +\sum_{n=0}^{l-1}\left(\begin{array}{c}
j-1+n \\
j-1
\end{array}\right) \lambda_{ \pm}^{n}\left(1-\lambda_{ \pm}\right)^{j} \Delta_{\mathbf{k}}^{l-n}\left(\mathbf{v}_{\mathbf{2}}\right) \Delta_{\mathbf{k}}^{j+n}\left(\mathbf{v}_{\mathbf{3}}^{ \pm}\right) .
\end{aligned}
$$

Another direct consequence of the two-point splitting lemma is the partial fractioning of the propagator of two different linear dependent vectors. If $\lambda_{1} \mathbf{v}_{\mathbf{1}}+\lambda_{2} \mathbf{v}_{\mathbf{2}}=\mathbf{0}$ with $\lambda_{1}+\lambda_{2} \neq 0$, then the line connecting $\mathbf{v}_{\mathbf{1}}$ and $\mathbf{v}_{\mathbf{2}}$ passes through the origin. Thus $\mathbf{0}$ is associated with them. As $\Delta_{\mathbf{k}}(\mathbf{0})=1$ the two-point splitting lemma can be used to perform a reduction of the number of propagators. Thus we have the two-point partial fractioning (see figure 4)

$$
\Delta_{\mathbf{k}}\left(\mathbf{v}_{\mathbf{1}}, \mathbf{v}_{\mathbf{2}}\right)=\frac{1}{\lambda_{1}+\lambda_{2}}\left[\lambda_{2} \Delta_{\mathbf{k}}\left(\mathbf{v}_{\mathbf{1}}\right)+\lambda_{1} \Delta_{\mathbf{k}}\left(\mathbf{v}_{\mathbf{2}}\right)\right],
$$

with two constraints $\lambda_{1} \mathbf{v}_{\mathbf{1}}+\lambda_{2} \mathbf{v}_{\mathbf{2}}=\mathbf{0}$ and $\lambda_{1}+\lambda_{2} \neq 0$. The most important special case of the two point partial fractioning is $\mathbf{v}_{\mathbf{2}}=-\mathbf{v}_{\mathbf{1}}$. Then one can write eq. (D.18) as

$$
\Delta_{\mathbf{k}}(\mathbf{v},-\mathbf{v})=\frac{1}{2}\left[\Delta_{\mathbf{k}}(\mathbf{v})+\Delta_{\mathbf{k}}(-\mathbf{v})\right] .
$$

\section{D.3 Three-point partial fractioning and general denominator reduction}

Eq. (D.18) gives a way to reduce the propagator of two linear dependent vectors to just one. Together with the two-point splitting lemma (8) we will geometrically construct 


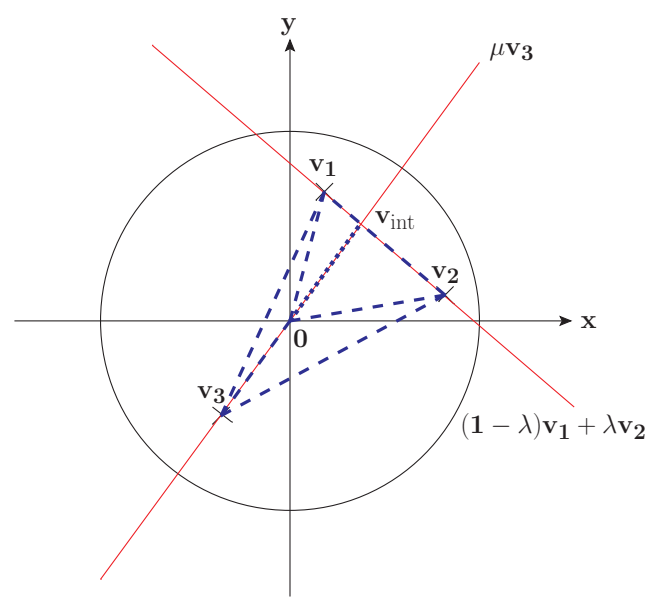

Figure 5. Graphical illustration of three-point partial fractioning.

the formula for the reduction of three propagators with linear dependent vectors to the sum of two propagators. The derived formula will be at the heart of the partial fraction decomposition for the NNLO real correction of the Drell-Yan angular distribution (which we plan to investigate in the near future in the context of small $Q_{T}$ resummation, compare ref. [42] and [43]).

Let us start with three linear dependent and pairwise linear independent vectors $\mathbf{v}_{\mathbf{1}}$, $\mathbf{v}_{\mathbf{2}}$ and $\mathbf{v}_{\mathbf{3}}$ satisfying

$$
\lambda_{1} \mathbf{v}_{\mathbf{1}}+\lambda_{2} \mathbf{v}_{\mathbf{2}}+\lambda_{3} \mathbf{v}_{\mathbf{3}}=\mathbf{0},
$$

with $\lambda_{1}+\lambda_{2}+\lambda_{3} \neq 0$. We want to derive an expression of the form

$$
\Delta_{\mathbf{k}}\left(\mathbf{v}_{\mathbf{1}}, \mathbf{v}_{\mathbf{2}}, \mathbf{v}_{\mathbf{3}}\right)=a_{1} \Delta_{\mathbf{k}}\left(\mathbf{v}_{\mathbf{1}}, \mathbf{v}_{\mathbf{2}}\right)+a_{2} \Delta_{\mathbf{k}}\left(\mathbf{v}_{\mathbf{1}}, \mathbf{v}_{\mathbf{3}}\right)+a_{3} \Delta_{\mathbf{k}}\left(\mathbf{v}_{\mathbf{2}}, \mathbf{v}_{\mathbf{3}}\right)
$$

with parameters $a_{1,2,3} \equiv a_{1,2,3}\left(\lambda_{1}, \lambda_{2}, \lambda_{3}\right)$ to be determined. In our graphical representation $\mathbf{v}_{\mathbf{1}}, \mathbf{v}_{\mathbf{2}}$ and $\mathbf{v}_{\mathbf{3}}$ form a triangle. We proceed in three steps depicted in figure 5.

First, we find the intersection of $\overline{\mathbf{v}_{\mathbf{1}} \mathbf{v}_{\mathbf{2}}}$ and $\overline{\mathbf{0 v}_{\mathbf{3}}}$ and name it $\mathbf{v}_{\text {int }} \cdot{ }^{4} \mathbf{v}_{\text {int }}$ is associated with $\mathbf{v}_{\mathbf{1}}$ by the two-point splitting lemma and therefore there exists a $\lambda$ such that

$$
\Delta_{\mathbf{k}}\left(\mathbf{v}_{\mathbf{1}}, \mathbf{v}_{\mathbf{2}}\right)=(1-\lambda) \Delta_{\mathbf{k}}\left(\mathbf{v}_{\mathbf{1}}, \mathbf{v}_{\text {int }}\right)+\lambda \Delta_{\mathbf{k}}\left(\mathbf{v}_{\mathbf{2}}, \mathbf{v}_{\text {int }}\right) .
$$

Therefore, upon multiplication of $\Delta_{\mathbf{k}}\left(\mathbf{v}_{\mathbf{1}}, \mathbf{v}_{\mathbf{2}}\right)$ with $\Delta_{\mathbf{k}}\left(\mathbf{v}_{\mathbf{3}}\right)$ it follows

$$
\Delta_{\mathbf{k}}\left(\mathbf{v}_{\mathbf{1}}, \mathbf{v}_{\mathbf{2}}, \mathbf{v}_{\mathbf{3}}\right)=(1-\lambda) \Delta_{\mathbf{k}}\left(\mathbf{v}_{\mathbf{1}}, \mathbf{v}_{\text {int }}, \mathbf{v}_{\mathbf{3}}\right)+\lambda \Delta_{\mathbf{k}}\left(\mathbf{v}_{\mathbf{2}}, \mathbf{v}_{\text {int }}, \mathbf{v}_{\mathbf{3}}\right) .
$$

Secondly, we observe that $\mathbf{v}_{\text {int }}$ is linear dependent on $\mathbf{v}_{\mathbf{3}}$, thus $\mathbf{v}_{\text {int }}=\mu \mathbf{v}_{\mathbf{3}}$ for some $\mu$. Hence, according to eq. (D.18) we can use the two-point partial fractioning

$$
\Delta_{\mathbf{k}}\left(\mathbf{v}_{\text {int }}, \mathbf{v}_{\mathbf{3}}\right)=\frac{1}{1-\mu} \Delta_{\mathbf{k}}\left(\mathbf{v}_{\mathbf{3}}\right)-\frac{\mu}{1-\mu} \Delta_{\mathbf{k}}\left(\mathbf{v}_{\text {int }}\right) .
$$

\footnotetext{
${ }^{4}$ In the case, where these lines are parallel such that no intersection exists, change the roles of $\mathbf{v}_{\mathbf{1}}, \mathbf{v}_{\mathbf{2}}$, and $\mathbf{v}_{\mathbf{3}}$ appropriately.
} 
Thus,

$$
\begin{aligned}
\Delta_{\mathbf{k}}\left(\mathbf{v}_{\mathbf{1}}, \mathbf{v}_{\mathbf{2}}, \mathbf{v}_{\mathbf{3}}\right)= & \frac{1-\lambda}{1-\mu} \Delta_{\mathbf{k}}\left(\mathbf{v}_{\mathbf{1}}, \mathbf{v}_{\mathbf{3}}\right)-\frac{(1-\lambda) \mu}{1-\mu} \Delta_{\mathbf{k}}\left(\mathbf{v}_{\mathbf{1}}, \mathbf{v}_{\text {int }}\right) \\
& +\frac{\lambda}{1-\mu} \Delta_{\mathbf{k}}\left(\mathbf{v}_{\mathbf{2}}, \mathbf{v}_{\mathbf{3}}\right)-\frac{\lambda \mu}{1-\mu} \Delta_{\mathbf{k}}\left(\mathbf{v}_{\mathbf{2}}, \mathbf{v}_{\text {int }}\right)
\end{aligned}
$$

with each of the summands corresponding to one of the four triangles with the corners $\left(\mathbf{0}, \mathbf{v}_{\mathbf{1}}, \mathbf{v}_{\mathbf{3}}\right),\left(\mathbf{0}, \mathbf{v}_{\mathbf{1}}, \mathbf{v}_{\text {int }}\right),\left(\mathbf{0}, \mathbf{v}_{\mathbf{2}}, \mathbf{v}_{\mathbf{3}}\right),\left(\mathbf{0}, \mathbf{v}_{\mathbf{2}}, \mathbf{v}_{\text {int }}\right)$.

In a third step we get rid of $\mathbf{v}_{\text {int }}$ by combining the triangles $\left(\mathbf{0}, \mathbf{v}_{\mathbf{1}}, \mathbf{v}_{\text {int }}\right)$ and $\left(\mathbf{0}, \mathbf{v}_{\mathbf{2}}, \mathbf{v}_{\text {int }}\right)$ with two-point splitting in reverse via eq. (D.7) such that

$$
\Delta_{\mathbf{k}}\left(\mathbf{v}_{\mathbf{1}}, \mathbf{v}_{\mathbf{2}}, \mathbf{v}_{\mathbf{3}}\right)=-\frac{\mu}{1-\mu} \Delta_{\mathbf{k}}\left(\mathbf{v}_{\mathbf{1}}, \mathbf{v}_{\mathbf{2}}\right)+\frac{1-\lambda}{1-\mu} \Delta_{\mathbf{k}}\left(\mathbf{v}_{\mathbf{1}}, \mathbf{v}_{\mathbf{3}}\right)+\frac{\lambda}{1-\mu} \Delta_{\mathbf{k}}\left(\mathbf{v}_{\mathbf{2}}, \mathbf{v}_{\mathbf{3}}\right)
$$

Solving the intersection equation

$$
\mu \mathbf{v}_{\mathbf{3}} \stackrel{!}{=} \lambda \mathbf{v}_{\mathbf{1}}+(1-\lambda) \mathbf{v}_{\mathbf{2}}
$$

with boundary condition (D.20) yields for $\lambda$ and $\mu$

$$
\lambda=\frac{\lambda_{1}}{\lambda_{1}+\lambda_{2}} \quad \text { and } \quad \mu=-\frac{\lambda_{3}}{\lambda_{1}+\lambda_{2}} .
$$

Plugging solution (D.28) into eq. (D.26) we have the three-point partial fractioning

$$
\Delta_{\mathbf{k}}\left(\mathbf{v}_{\mathbf{1}}, \mathbf{v}_{\mathbf{2}}, \mathbf{v}_{\mathbf{3}}\right)=\frac{1}{\lambda_{1}+\lambda_{2}+\lambda_{3}}\left[\lambda_{3} \Delta_{\mathbf{k}}\left(\mathbf{v}_{\mathbf{1}}, \mathbf{v}_{\mathbf{2}}\right)+\lambda_{2} \Delta_{\mathbf{k}}\left(\mathbf{v}_{\mathbf{1}}, \mathbf{v}_{\mathbf{3}}\right)+\lambda_{1} \Delta_{\mathbf{k}}\left(\mathbf{v}_{\mathbf{2}}, \mathbf{v}_{\mathbf{3}}\right)\right],
$$

with boundary conditions $\lambda_{1} \mathbf{v}_{\mathbf{1}}+\lambda_{2} \mathbf{v}_{\mathbf{2}}+\lambda_{3} \mathbf{v}_{\mathbf{3}}=\mathbf{0}$ and $\lambda_{1}+\lambda_{2}+\lambda_{3} \neq 0$.

After the illustration of the application of two-point splitting lemma to partialfractioning of products of two and three propagators, we are in the position to extend it to the general case of the product of $n$ propagators with $n$ being arbitrary. Therefore, we formulate the generalized reduction of products of linear propagators:

If $\sum_{i=1}^{n} \lambda_{i} \mathbf{v}_{\mathbf{i}}=\mathbf{0}$ with $\lambda \equiv \sum_{i=1}^{n} \lambda_{i} \neq 0, n \in \mathbb{N}, \lambda_{i} \in \mathbb{C}$, then it holds

$$
\prod_{i=1}^{n} \Delta_{\mathbf{k}}\left(\mathbf{v}_{\mathbf{i}}\right)=\frac{1}{\lambda} \sum_{j=1}^{n} \lambda_{j} \prod_{i=1}^{n \neq j} \Delta_{\mathbf{k}}\left(\mathbf{v}_{\mathbf{i}}\right)
$$

The proof of the identity (D.30) is based on the linearity of the propagator and is straightforward. In particular, using $\mathbf{0}=\sum_{j=1}^{n} \lambda_{j} \mathbf{v}_{\mathbf{j}}$ one gets

$$
\lambda=\sum_{j=1}^{n} \lambda_{j}=\sum_{j=1}^{n} \lambda_{j}\left(1-\mathbf{v}_{\mathbf{j}} \mathbf{k}\right)=\sum_{j=1}^{n} \lambda_{j} \Delta_{\mathbf{k}}^{-1}\left(\mathbf{v}_{\mathbf{j}}\right)
$$


Therefore,

$$
\begin{aligned}
\Delta_{\mathbf{k}}\left(\mathbf{v}_{\mathbf{1}}, \mathbf{v}_{\mathbf{2}}, \cdots, \mathbf{v}_{\mathbf{n}}\right)= & \prod_{i=1}^{n} \Delta_{\mathbf{k}}\left(\mathbf{v}_{\mathbf{i}}\right)=\frac{1}{\lambda} \sum_{j=1}^{n} \lambda_{j} \prod_{i=1}^{n} \Delta_{\mathbf{k}}\left(\mathbf{v}_{\mathbf{i}}\right) \\
= & \frac{1}{\lambda} \sum_{j=1}^{n} \lambda_{j} \prod_{i=1}^{n} \Delta_{\mathbf{k}}^{-1}\left(\mathbf{v}_{\mathbf{j}}\right) \Delta_{\mathbf{k}}\left(\mathbf{v}_{\mathbf{i}}\right)=\frac{1}{\lambda} \sum_{j=1}^{n} \lambda_{j} \prod_{i=1}^{n \neq j} \Delta_{\mathbf{k}}\left(\mathbf{v}_{\mathbf{i}}\right) \\
= & \frac{1}{\lambda}\left[\lambda_{1} \Delta_{\mathbf{k}}\left(\mathbf{v}_{\mathbf{2}}, \mathbf{v}_{\mathbf{3}}, \cdots, \mathbf{v}_{\mathbf{n}}\right)+\lambda_{2} \Delta_{\mathbf{k}}\left(\mathbf{v}_{\mathbf{1}}, \mathbf{v}_{\mathbf{3}}, \cdots, \mathbf{v}_{\mathbf{n}}\right)\right. \\
& \left.+\ldots+\lambda_{n} \Delta_{\mathbf{k}}\left(\mathbf{v}_{\mathbf{1}}, \mathbf{v}_{\mathbf{2}}, \cdots, \mathbf{v}_{\mathbf{n}-\mathbf{1}}\right)\right]
\end{aligned}
$$

Geometrically eq. (D.30) follows from the fact that $\mathbf{0}$ is in the $n$-1-dimensional hyperplane spanned by the vectors $\mathbf{v}_{\mathbf{i}}$ and, thus it can be associated with them repeatedly using the twopoint splitting lemma. Of course, the general reduction of linear propagators contains twoand three-point partial fractioning as mere special cases. However, we think that the presented inductive approach is more pedagogically suited to build up an geometric intuition.

\section{D.4 Propagators to negative integer powers}

In practical calculations one is also confronted with negative integer powers $j \in \mathbb{Z}_{\leq 0}$ of massive propagators. Those can always be made massless by simple scaling. It holds

$$
\begin{aligned}
\Delta_{\mathbf{k}}^{-1}\left(\mathbf{v}_{\mathbf{1}}\right) & =1-\mathbf{v}_{\mathbf{1}} \cdot \mathbf{k}=1-\left|\mathbf{v}_{\mathbf{1}}\right|+\left|\mathbf{v}_{\mathbf{1}}\right| \Delta_{\mathbf{k}}^{-1}\left(\frac{\mathbf{v}_{\mathbf{1}}}{\left|\mathbf{v}_{\mathbf{1}}\right|}\right) \\
& =1-\sqrt{1-v_{11}}+\sqrt{1-v_{11}} \Delta_{\mathbf{k}}^{-1}\left(\hat{\mathbf{v}}_{\mathbf{1}}\right)
\end{aligned}
$$

with $\hat{\mathbf{v}}_{\mathbf{1}}=\frac{\mathbf{v}_{\mathbf{1}}}{\left|\mathbf{v}_{\mathbf{1}}\right|}=\frac{\mathbf{v}_{\mathbf{1}}}{\sqrt{1-v_{11}}}$. Contracting with another vector $\mathbf{v}_{\mathbf{2}}$ gives the scalar product

$$
\hat{v}_{12}=1-\hat{\mathbf{v}}_{\mathbf{1}} \cdot \mathbf{v}_{\mathbf{2}}=1-\frac{\mathbf{v}_{\mathbf{1}} \cdot \mathbf{v}_{\mathbf{2}}}{\sqrt{1-v_{11}}}=1-\frac{1-v_{12}}{\sqrt{1-v_{11}}}
$$

Eq. (D.33) is straightforwardly generalized to $j \in \mathbb{Z}_{\leq 0}$ by application of the Binomial theorem

$$
\begin{aligned}
\Delta_{\mathbf{k}}^{j}\left(\mathbf{v}_{\mathbf{1}}\right) & =\left(1-\sqrt{1-v_{11}}+\sqrt{1-v_{11}} \Delta_{\mathbf{k}}^{-1}\left(\hat{\mathbf{v}}_{\mathbf{1}}\right)\right)^{-j} \\
& =\sum_{n=0}^{-j}\left(\begin{array}{c}
-j \\
n
\end{array}\right)\left(1-\sqrt{1-v_{11}}\right)^{-j-n}\left(1-v_{11}\right)^{n / 2} \Delta_{\mathbf{k}}^{-n}\left(\hat{\mathbf{v}}_{\mathbf{1}}\right) .
\end{aligned}
$$

This equation allows to generalize the two-mass splitting to all $j, l \in \mathbb{Z}$. Another practical application is that one can reduce two-mass angular integral to one-mass one in case of 
$j \in \mathbb{Z}_{\leq 0}$. In particular, for that case it holds

$$
\begin{aligned}
I_{j, l}^{(2)}\left(v_{12}, v_{11}, v_{22} ; \varepsilon\right) & =\int \mathrm{d} \Omega_{k_{1} k_{2}} \Delta_{\mathbf{k}}^{j}\left(\mathbf{v}_{\mathbf{1}}\right) \Delta_{\mathbf{k}}^{l}\left(\mathbf{v}_{\mathbf{2}}\right) \\
& =\sum_{n=0}^{-j}\left(\begin{array}{c}
-j \\
n
\end{array}\right)\left(1-\sqrt{1-v_{11}}\right)^{-j-n}\left(1-v_{11}\right)^{n / 2} \int \mathrm{d} \Omega_{k_{1} k_{2}} \Delta_{\mathbf{k}}^{-n}\left(\hat{\mathbf{v}}_{\mathbf{1}}\right) \Delta_{\mathbf{k}}^{l}\left(\mathbf{v}_{\mathbf{2}}\right) \\
& =\sum_{n=0}^{-j}\left(\begin{array}{c}
-j \\
n
\end{array}\right)\left(1-\sqrt{1-v_{11}}\right)^{-j-n}\left(1-v_{11}\right)^{n / 2} I_{l,-n}^{(1)}\left(1-\frac{1-v_{12}}{\sqrt{1-v_{11}}}, v_{22} ; \varepsilon\right) .
\end{aligned}
$$

Equivalently for $l \in \mathbb{Z}_{\leq 0}$

$$
\begin{aligned}
I_{j, l}^{(2)}\left(v_{12}, v_{11}, v_{22} ; \varepsilon\right)= & \sum_{n=0}^{-l}\left(\begin{array}{c}
-l \\
n
\end{array}\right)\left(1-\sqrt{1-v_{22}}\right)^{-l-n}\left(1-v_{22}\right)^{n / 2} \\
& \times I_{j,-n}^{(1)}\left(1-\frac{1-v_{12}}{\sqrt{1-v_{22}}}, v_{11} ; \varepsilon\right)
\end{aligned}
$$

\section{D.5 Connection to physical propagators}

Let us consider a situation with fixed momenta $p_{1}, p_{2}, \ldots, p_{n}$ and a PSI over the external momenta $k_{1}$ and $k_{2}$, where $\sum_{j=1}^{n} p_{j}=k_{1}+k_{2}$ and establish the connection of physical propagators with the scaled propagators occurring in the derived partial fractioning formulas. We use the CMF for the angular integration momenta $\left(\mathbf{k}_{\mathbf{1}}+\mathbf{k}_{\mathbf{2}}=0\right)$ and scale all momenta by their corresponding energy component:

$$
k_{1}=E_{k}(1, \mathbf{k}), \quad k_{2}=E_{k}(1,-\mathbf{k}), \quad p_{i}=E_{i}\left(1, \mathbf{v}_{\mathbf{i}}\right) \text {. }
$$

Also we suppose that $k_{1,2}$ momenta are on their mass-shell and for simplicity we assume that they correspond to the same mass: $k_{1,2}^{2}=m_{k}^{2}$. Considering generic physical propagator depending on external $p_{i}$ and angular loop $\left(k_{1}, k_{2}\right)$ momenta

$$
\Delta_{\text {phys }}\left(k_{1,2}, p_{i}, m\right)=\frac{1}{\left(k_{1,2}-p_{i}\right)^{2}-m^{2}},
$$

we can always express it in terms of scaled propagator $\Delta_{\mathbf{k}}\left( \pm \overline{\mathbf{v}}_{\mathbf{i}}\right)$ :

$$
\Delta_{\text {phys }}\left(k_{1,2}, p_{i}, m\right)=\frac{1}{p_{i}^{2}-m^{2}+m_{k}^{2}-2 E_{i} E_{k}} \Delta_{\mathbf{k}}\left( \pm \overline{\mathbf{v}}_{\mathbf{i}}\right),
$$

where we introduced the scaled vector

$$
\overline{\mathbf{v}}_{\mathbf{i}}=\frac{\mathbf{v}_{\mathbf{i}}}{\frac{p_{i}^{2}-m^{2}+m_{k}^{2}}{2 E_{i} E_{k}}-1} .
$$

Note the two vectors $v_{i}=\left(1, \mathbf{v}_{\mathbf{i}}\right)$ and $\bar{v}_{i}=\left(1, \overline{\mathbf{v}}_{\mathbf{i}}\right)$ are differed only by spatial part [see eq. (D.41)]. So, up to an angular independent factor we can express any physical propagator of the discussed form as a scaled propagator with a suitably chosen scaled vector. 


\section{D.6 Example: Drell-Yan double real corrections kinematics}

To demonstrate how we can bring our general considerations about partial fractioning to good use in a physical situation we consider the kinematics schematically shown in figure 6 . This is the kinematics of the double real corrections for the Drell-Yan process. Conservation of momentum implies that $p_{1}+p_{2}=q+k_{1}+k_{2}$. For the PSI $P_{\text {ext }}=p_{1}+p_{2}-q$. Hence, the angular PSI is defined in the CMF of $p_{1}, p_{2}$, and $-q$ (or equivalently in the CMF of $k_{1}$ and $k_{2}$ ):

$$
\mathbf{p}_{1}+\mathbf{p}_{2}-\mathbf{q}=0 .
$$

These three vectors are linear dependent and thus lie in a common plane, defining the $\left(x_{D-1}, x_{D}\right)$-plane. We can then depict the kinematics in a three dimensional slice of $D$ dimensional space-time (see figure 7). Since all involved particles are massless, we have propagators of the general form

$$
\frac{1}{\left(k_{1,2}-p\right)^{2}+i 0},
$$

where $p$ is one of the fixed momenta. In the rest frame of $p_{1}, p_{2}$, and $-q$ with the $x_{D}$-axis chosen in the direction of $\mathbf{p}_{\mathbf{1}}$ the vectors can be parametrized as

$$
\begin{aligned}
k_{1} & =\frac{\sqrt{s_{2}}}{2}\left(1, \mathbf{v}_{\mathbf{k}}\right)=\frac{\sqrt{s_{2}}}{2}\left(1, \ldots, \sin \theta_{1} \cos \theta_{2}, \cos \theta_{1}\right), \\
k_{2} & =\frac{\sqrt{s_{2}}}{2}\left(1, \mathbf{v}_{\mathbf{k}}\right)=\frac{\sqrt{s_{2}}}{2}\left(1, \ldots,-\sin \theta_{1} \cos \theta_{2},-\cos \theta_{1}\right), \\
p_{1} & =\frac{s_{2}-u}{2 \sqrt{s_{2}}}\left(1, \mathbf{v}_{\mathbf{1}}\right)=\frac{s_{2}-u}{2 \sqrt{s_{2}}}\left(1, \mathbf{0}_{D-3}, 0,1\right), \\
p_{2} & =\frac{s_{2}-t}{2 \sqrt{s_{2}}}\left(1, \mathbf{v}_{\mathbf{2}}\right)=\frac{s_{2}-t}{2 \sqrt{s_{2}}}\left(1, \mathbf{0}_{D-3}, \sin \vartheta, \cos \vartheta\right), \\
q & =-\frac{t+u}{2 \sqrt{s_{2}}}\left(1, \mathbf{v}_{\mathbf{q}}\right)=-\frac{t+u}{2 \sqrt{s_{2}}}\left(1, \mathbf{0}_{D-3}, \frac{t-s_{2}}{t+u} \sin \vartheta, \frac{u-s_{2}}{t+u}+\frac{t-s_{2}}{t+u} \cos \vartheta\right),
\end{aligned}
$$

with

$$
\cos \vartheta=1-\frac{2 s s_{2}}{\left(s_{2}-u\right)\left(s_{2}-t\right)}
$$

where

$$
s=\left(p_{1}+p_{2}\right)^{2}, \quad t=\left(p_{1}-q\right)^{2}, \quad u=\left(p_{2}-q\right)^{2}, \quad s_{2}=\left(k_{1}+k_{2}\right)^{2} .
$$

By conservation of four momentum it holds $Q^{2}=q^{2}=s+t+u-s_{2}$. It is also useful to write the relations between Mandelstam variables and components of transverse momentum in light-cone frame $q=\left(Q^{+}, Q^{-}, Q_{T}\right)$, where

$$
\begin{aligned}
Q^{ \pm} & =\frac{Q^{0} \pm Q^{3}}{\sqrt{2}}, \quad q^{2}=Q^{2}=2 Q^{+} Q^{-}-Q_{T}^{2}, \\
Q_{T}^{2} & =\frac{\lambda\left(s, s_{2}, Q^{2}\right)}{4 s_{2}}=\frac{u t-s_{2} Q^{2}}{s}, \\
\cos \vartheta & =\frac{Q_{T}^{2}-s_{2}}{Q_{T}^{2}+s_{2}}, \quad \sin \vartheta=\frac{2 Q_{T} \sqrt{s_{2}}}{Q_{T}^{2}+s_{2}}, \quad \sqrt{s_{2}}=Q_{T} \tan \frac{\vartheta}{2} .
\end{aligned}
$$




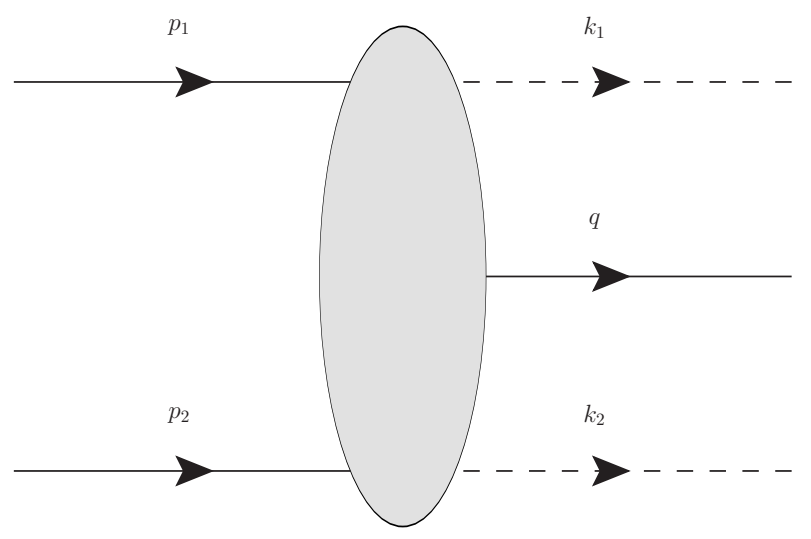

Figure 6. Kinematics for double real corrections of the Drell-Yan process. $p_{1}$ and $p_{2}$ are incoming momenta, $q$ is outgoing. The PSI is performed over the dashed momenta $k_{1}$ and $k_{2}$.

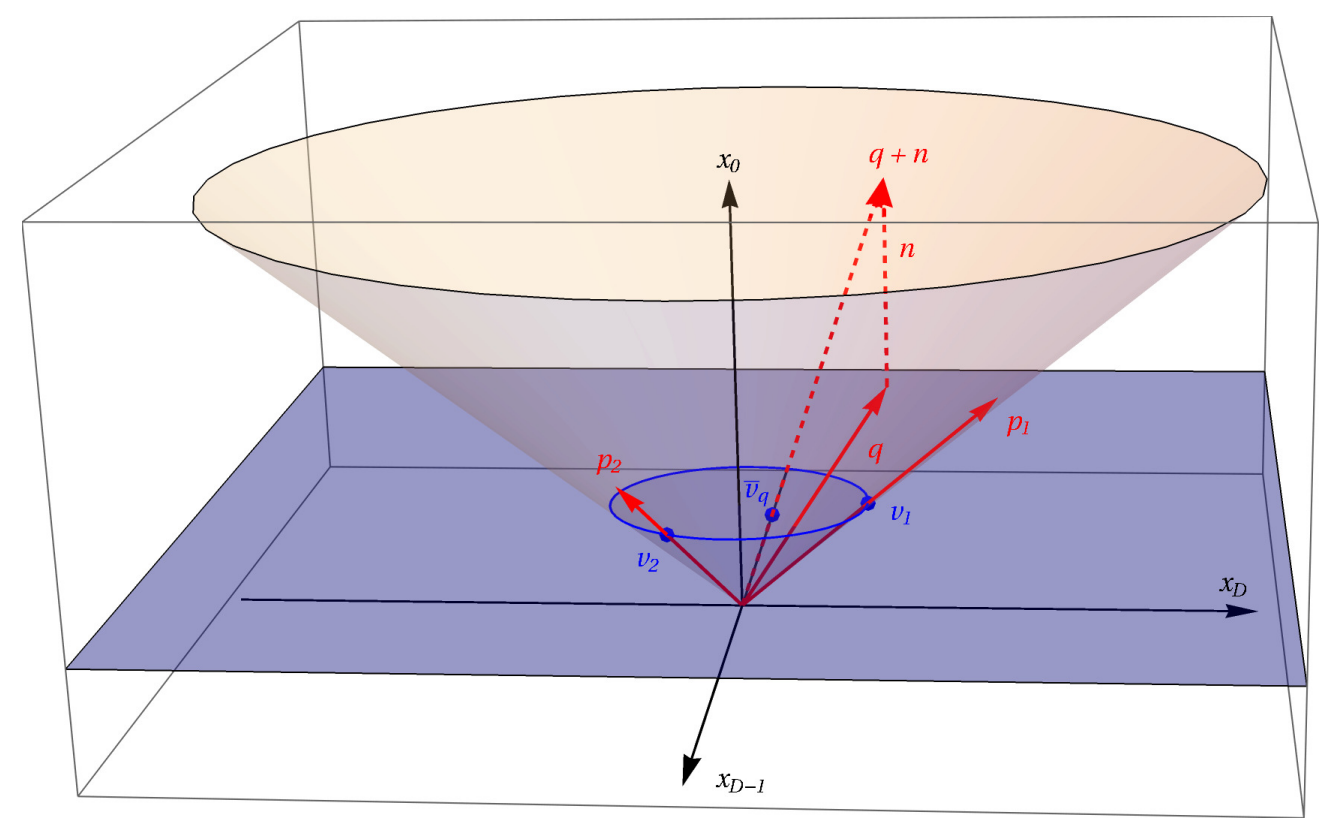

Figure 7. The picture shows the $0 \equiv x_{1} \equiv \cdots \equiv x_{D-2}$ slice of Minkowski space. The lightcone is shown in light yellow. The external momenta are depicted as red arrows, $p_{1}$ and $p_{2}$ are massless and hence on the light-cone, $q$ has mass $Q^{2}$ and is inside the light-cone. The scaled momenta $v$, marked by blue dots, are in the blue $x_{0} \equiv 1$ plane. The blue circle marks the intersection of the light-cone with the $x_{0} \equiv 1$ plane. The dashed red momenta illustrate the construction of $\bar{v}_{q}$. 


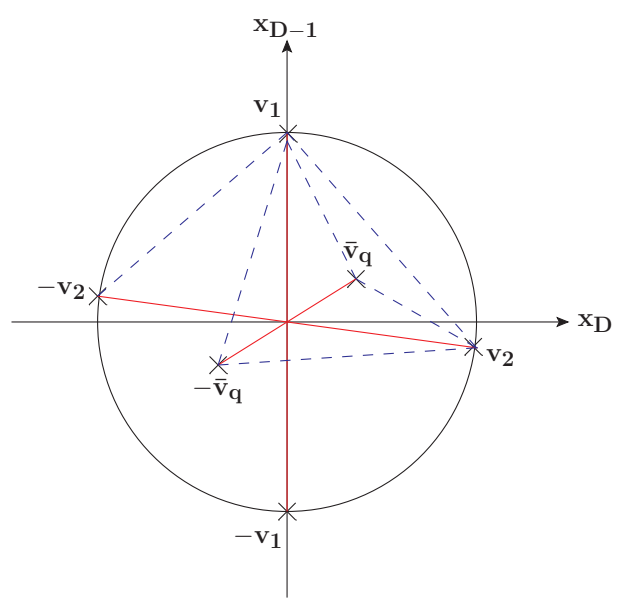

Figure 8. Drell-Yan kinematics in terms of scaled vectors. Red lines indicate pairs of linear dependent vectors. The dashed blue lines mark contributions to genuinely different PSIs.

Introducing scaled momenta

$$
\begin{array}{ll}
v_{1}=\frac{p_{1}}{E_{1}}=\left(1, \mathbf{v}_{\mathbf{1}}\right), & v_{2}=\frac{p_{2}}{E_{2}}=\left(1, \mathbf{v}_{\mathbf{2}}\right), \\
v_{q}=\frac{q}{E_{q}}=\left(1, \mathbf{v}_{\mathbf{q}}\right), & \bar{v}_{q}=\left(1, \overline{\mathbf{v}}_{\mathbf{q}}\right),
\end{array}
$$

where

$$
\overline{\mathbf{v}}_{\mathbf{q}}=\frac{\mathbf{v}_{\mathbf{q}}}{1+\frac{Q^{2}}{2 E_{k} E_{q}}}=\mathbf{v}_{\mathbf{q}} \frac{t+u}{t+u-2 Q^{2}}
$$

the propagators become

$$
\begin{aligned}
\frac{1}{t_{1,2}} & \equiv \frac{1}{\left(k_{1,2}-p_{1}\right)^{2}}=\frac{1}{-2 k_{1,2} \cdot p_{1}}=-\frac{2}{s_{2}-u} \Delta_{\mathbf{k}}\left( \pm \mathbf{v}_{\mathbf{1}}\right) \\
\frac{1}{u_{1,2}} & \equiv \frac{1}{\left(k_{1,2}-p_{2}\right)^{2}}=\frac{1}{-2 k_{1,2} \cdot p_{2}}=-\frac{2}{s_{2}-t} \Delta_{\mathbf{k}}\left( \pm \mathbf{v}_{\mathbf{2}}\right) \\
\frac{1}{w_{1,2}} & \equiv \frac{1}{\left(k_{1,2}+q\right)^{2}}=\frac{1}{2 k_{1,2} \cdot q+Q^{2}}=\frac{2}{2 Q^{2}-t-u} \Delta_{\mathbf{k}}\left( \pm \overline{\mathbf{v}}_{\mathbf{q}}\right) .
\end{aligned}
$$

The construction of the scaled momenta is depicted in figure 7. The general denominators appearing in our calculations will have the form

$$
\begin{aligned}
\frac{1}{t_{1}^{n_{1}} t_{2}^{n_{2}} u_{1}^{n_{3}} u_{2}^{n_{4}} w_{1}^{n_{5}} w_{2}^{n_{6}}}= & \left(-\frac{2}{s_{2}-u}\right)^{n_{1}+n_{2}}\left(-\frac{2}{s_{2}-t}\right)^{n_{3}+n_{4}}\left(\frac{2}{2 Q^{2}-t-u}\right)^{n_{5}+n_{6}} \\
& \times \Delta_{\mathbf{k}}^{n_{1}}\left(\mathbf{v}_{\mathbf{1}}\right) \Delta_{\mathbf{k}}^{n_{2}}\left(-\mathbf{v}_{\mathbf{1}}\right) \Delta_{\mathbf{k}}^{n_{3}}\left(\mathbf{v}_{\mathbf{2}}\right) \Delta_{\mathbf{k}}^{n_{4}}\left(-\mathbf{v}_{\mathbf{2}}\right) \Delta_{\mathbf{k}}^{n_{5}}\left(\overline{\mathbf{v}}_{\mathbf{q}}\right) \Delta_{\mathbf{k}}^{n_{6}}\left(-\overline{\mathbf{v}}_{\mathbf{q}}\right) .
\end{aligned}
$$

In terms of the pictures from our general considerations, the situation in the $x_{0} \equiv 1$ plane looks as figure 8 . 
We can decompose eq. (D.53) into a sum of terms were each one contains a pair of linearly independent vectors by:

1. performing two-point partial fractioning between the pairs of linearly dependent vectors;

2. doing three-point partial fractioning between the remaining three vectors.

The $\lambda$ used for three point partial fractioning can be read of using the condition

$$
\mathbf{0}=\left(s_{2}-u\right) \mathbf{v}_{\mathbf{1}}+\left(s_{2}-t\right) \mathbf{v}_{\mathbf{2}}+\left(2 Q^{2}-t-u\right) \overline{\mathbf{v}}_{\mathbf{q}}=2 \sqrt{s_{2}}\left(\mathbf{p}_{1}+\mathbf{p}_{2}-\mathbf{q}\right)
$$

which is the direct consequence of eq. (D.42).

Expressing the numerator originating from some Dirac trace as a polynomial of the two corresponding Mandelstam variables we can simplify our angular integrals. In particular, the numerator simply decreases the powers of propagators in denominator. All that we need is to complete the calculation of the following set of integrals

$$
\begin{aligned}
I_{j, l}^{(0)}\left(u_{12}^{ \pm} ; \varepsilon\right) & =\int \mathrm{d} \Omega_{k_{1}, k_{2}} \Delta_{\mathbf{k}}^{j}\left(\mathbf{v}_{\mathbf{1}}\right) \Delta_{\mathbf{k}}^{l}\left(\mp \mathbf{v}_{\mathbf{2}}\right)=\int \mathrm{d} \Omega_{k_{1}, k_{2}} \Delta_{\mathbf{k}}^{j}\left(-\mathbf{v}_{\mathbf{1}}\right) \Delta_{\mathbf{k}}^{l}\left( \pm \mathbf{v}_{\mathbf{2}}\right) \\
I_{j, l}^{(1)}\left(u_{i \bar{q}}^{ \pm}, v_{\bar{q} \bar{q}} ; \varepsilon\right) & =\int \mathrm{d} \Omega_{k_{1}, k_{2}} \Delta_{\mathbf{k}}^{j}\left(\overline{\mathbf{v}}_{\mathbf{q}}\right) \Delta_{\mathbf{k}}^{l}\left(\mp \mathbf{v}_{\mathbf{i}}\right)=\int \mathrm{d} \Omega_{k_{1}, k_{2}} \Delta_{\mathbf{k}}^{j}\left(-\overline{\mathbf{v}}_{\mathbf{q}}\right) \Delta_{\mathbf{k}}^{l}\left( \pm \mathbf{v}_{\mathbf{i}}\right)
\end{aligned}
$$

where $i=1,2$ and

$$
\begin{array}{ll}
u_{12}^{ \pm}=1 \pm\left(1-v_{12}\right), & u_{i \bar{q}}^{ \pm}=1 \pm\left(1-v_{i \bar{q}}\right), \\
v_{12}=1-\cos \vartheta=\frac{2 s s_{2}}{\left(s_{2}-u\right)\left(s_{2}-t\right)}, & v_{\bar{q} \bar{q}}=\frac{4 s Q^{2}}{\left(s+Q^{2}-s_{2}\right)^{2}} \\
v_{1 \bar{q}}=2\left[1+\frac{s u}{\left(s_{2}-u\right)\left(s+Q^{2}-s_{2}\right)}\right], & v_{2 \bar{q}}=2\left[1+\frac{s t}{\left(s_{2}-t\right)\left(s+Q^{2}-s_{2}\right)}\right],
\end{array}
$$

for integer valued $j$ and $l$. These are exactly the massless and single-massive two-denominator integrals presented in the main text. Note that the integrals depending on the arguments $v_{1 \bar{q}}$ and $v_{2 \bar{q}}$ are related by a crossing symmetry, i.e. by interchange $t \leftrightarrow u$. All in all, we were able to write down all potentially appearing integrals for the process without any concrete calulation.

\section{E Consistency of the hypergeometric representation of angular integrals}

In this appendix we present further details regarding hypergeometric representation of the general two denominator angular integrals in $D$ dimensions. First, we check consistency of the master formula for the two denominator integral in terms of the Lauricella function derived in eq. (3.81) with known special results, e.g. derived in ref. [48]. 


\section{E.1 Double massive integral in four dimensions}

To perform the check of eq. (3.81) we start with the double massive integral in four dimensions. In particular, for the special case $j=l=1$ and $\varepsilon=0$ we recover the well-known four-dimensional expression for $I_{1,1}$ using the chain of the reduction identities

$$
\begin{aligned}
F_{B}^{(3)}\left(a_{1}, a_{2}, a_{3}, b_{1}, b_{2}, 0, c, x_{1}, x_{2}, x_{3}\right) & =F_{3}\left(a_{1}, a_{2}, b_{1}, b_{2}, c, x_{1}, x_{2}\right), \\
F_{3}\left(a_{1}, a_{2}, b_{1}, b_{2}, a_{1}+a_{2}, x_{1}, x_{2}\right) & =\left(1-x_{2}\right)^{-b_{2}} F_{1}\left(a_{1}, b_{1}, b_{2}, a_{1}+a_{2}, x_{1}, \frac{x_{2}}{x_{2}-1}\right), \\
F_{1}\left(a, b_{1}, b_{2}, b_{1}+b_{2}, x_{1}, x_{2}\right) & =\left(1-x_{2}\right)^{-a}{ }_{2} F_{1}\left(a, b_{1}, b_{1}+b_{2}, \frac{x_{1}-x_{2}}{1-x_{2}}\right),
\end{aligned}
$$

the quadratic hypergeometric transformation

$$
{ }_{2} F_{1}\left(\frac{a}{2}, \frac{a+1}{2}, b+\frac{1}{2}, x\right)=(1+\sqrt{x})^{-a}{ }_{2} F_{1}\left(a, b, 2 b, 2-\frac{2}{1+\sqrt{x}}\right),
$$

and

$$
{ }_{2} F_{1}(1,1,2, x)=\sum_{n=0}^{\infty} \frac{(1)_{n}(1)_{n}}{(2)_{n}} \frac{x^{n}}{n !}=\sum_{n=0}^{\infty} \frac{x^{n}}{n+1}=-\frac{\log (1-x)}{x} .
$$

Starting with formula (3.81), where we interchange the symmetric parameters $a_{1}$ and $b_{1}$, we straightforwardly obtain

$$
\begin{aligned}
I_{1,1}\left(v_{12}, v_{11}, v_{22} ; 0\right) & \stackrel{(3.81)}{=} \frac{2 \pi}{v_{12}} F_{B}^{(3)}\left(1, \frac{1}{2}, \frac{1}{2}, \frac{1}{2}, 1,0, \frac{3}{2} ; 1-\frac{v_{11}}{v_{12}}, 1-\frac{v_{22}}{v_{12}}, 1-v_{12}\right) \\
& \stackrel{(E .1)}{=} \frac{2 \pi}{v_{12}} F_{3}\left(1, \frac{1}{2}, \frac{1}{2}, 1, \frac{3}{2}, 1-\frac{v_{11}}{v_{12}}, 1-\frac{v_{22}}{v_{12}}\right) \\
& \stackrel{(E .2)}{=} \frac{2 \pi}{v_{12}} \frac{v_{12}}{v_{22}} F_{1}\left(1, \frac{1}{2}, 1, \frac{3}{2}, 1-\frac{v_{11}}{v_{12}}, 1-\frac{v_{12}}{v_{22}}\right) \\
& \stackrel{(E .3)}{=} \frac{2 \pi}{v_{12}}{ }_{2} F_{1}\left(1, \frac{1}{2}, \frac{3}{2} ; 1-\frac{v_{11} v_{22}}{v_{12}^{2}}\right) \\
& \stackrel{(E .4)}{=} \frac{2 \pi}{v_{12}+\sqrt{v_{12}^{2}-v_{11} v_{22}}}{ }_{2} F_{1}\left(1,1,2,1-\frac{v_{12}}{v_{12}}+\sqrt{v_{12}^{2}-v_{11} v_{22}}\right. \\
& \left.\stackrel{(E .5)}{=} \frac{\pi}{\sqrt{v_{12}^{2}-v_{11} v_{22}}}\right)
\end{aligned}
$$

\section{E.2 Single massive integral}

In consideration of single massive integral we use two important indentities

$$
\begin{aligned}
& F_{1}\left(a, b, b, c, \frac{y+\sqrt{y+(1-y) x}}{y-1}, \frac{y-\sqrt{y+(1-y) x}}{y-1}\right) \\
& =\frac{(1-y)^{b}}{B(a, c-a)} \int_{0}^{1} \mathrm{~d} t t^{a-1}(1-t)^{c-a-1}\left(1-x t^{2}-y(1-t)^{2}\right)^{-b} \\
& =(1-y)^{b} \sum_{m, n=0}^{\infty} \frac{(a)_{2 m}(c-a)_{2 n}(b)_{m+n}}{(c)_{2 m+2 n}} \frac{x^{m}}{m !} \frac{y^{n}}{n !}
\end{aligned}
$$


and

$$
\sum_{n=0}^{\infty} \frac{(l)_{2 n} 4^{-n}}{\left(\frac{3}{2}-\varepsilon\right)_{m+n+p} n !}={ }_{2} F_{1}\left(\frac{l}{2}, \frac{l+1}{2}, \frac{3}{2}-\varepsilon, 1\right) \frac{(1-l-\varepsilon)_{m+p}}{(2-l-2 \varepsilon)_{2 m+2 p}} 4^{m+p} .
$$

The identity (E.7) is derived using integral representations for the Appell and gamma functions and the bivariate generating function

$$
\left(1-x t^{2}-y(1-t)^{2}\right)^{-b}=\sum_{m, n=0}^{\infty}(b)_{m+n} \frac{x^{m}}{m !} \frac{y^{n}}{n !} t^{2 m}(1-t)^{2 n},
$$

and then using duplication formula for the Pochhammer symbols

$$
(x)_{n}\left(x+\frac{1}{2}\right)_{n}=2^{-2 n}(2 x)_{2 n} .
$$

The second identity (E.8) is proved using the Pochhammer shift identities

$$
\begin{aligned}
(x)_{m+n} & =(x+m)_{n}(x)_{m}, \\
(x)_{m+n}(x-n)_{n} & =(x+m)_{n}(x-n)_{m+n},
\end{aligned}
$$

duplication identity (E.10), and the Pochhammer-Gauss summation formula

$$
\sum_{n=0}^{\infty} \frac{(a)_{n}(b)_{n}}{(c)_{n} n !}={ }_{2} F_{1}(a, b, c, 1)=\frac{(c-a)_{a}}{(c-a-b)_{a}} .
$$

Here, eq. (E.12) is a generalization of eq. (E.11) derived by multiplying of 1.h.s. and r.h.s. of eq. (E.11) by $(x-n)_{n}$ :

$$
(x)_{m+n}(x-n)_{n}=(x+m)_{n} \underbrace{(x)_{m}(x-n)_{n}}_{=(x-n)_{m+n}}=(x+m)_{n}(x-n)_{m+n} .
$$

Eq. (E.13) is the combination of two formulas - power expansion of Gauss function (3.31) and Gauss summation theorem (3.37).

In particular, eq. (E.7) is derived as:

$$
\begin{aligned}
& \sum_{n=0}^{\infty} \frac{(l)_{2 n} 4^{-n}}{\left(\frac{3}{2}-\varepsilon\right)_{m+n+p} n !} \stackrel{\text { (E.10), }}{=}{ }^{\text {E.11 })} \frac{1}{\left(\frac{3}{2}-\varepsilon\right)_{m+p}} \sum_{n=0}^{\infty} \frac{\left(\frac{l}{2}\right)_{n}\left(\frac{l+1}{2}\right)_{n}}{\left(\frac{3}{2}-\varepsilon+m+p\right)_{n} n !} \\
& \stackrel{(\mathrm{E} .13)}{=} \frac{1}{\left(\frac{3}{2}-\varepsilon\right)_{m+p}} \frac{\left(\frac{3}{2}-\frac{l}{2}+m+p-\varepsilon\right)_{\frac{l}{2}}}{(1-l+m+p-\varepsilon)_{\frac{l}{2}}}
\end{aligned}
$$

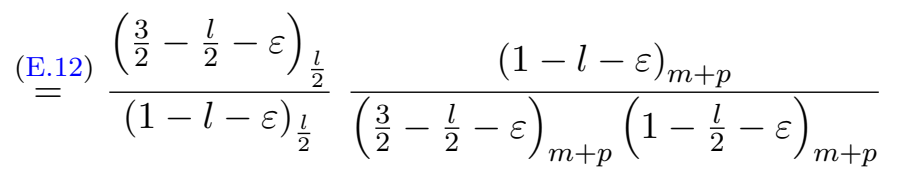

$$
\begin{aligned}
& \stackrel{\text { (E.10) }}{=}{ }_{2} F_{1}\left(\frac{l}{2}, \frac{l+1}{2}, \frac{3}{2}-\varepsilon, 1\right) \frac{(1-l-\varepsilon)_{m+p}}{(2-l-2 \varepsilon)_{2 m+2 p}} 4^{m+p} \text {. }
\end{aligned}
$$

Note, in the step from the second to the third line of eq. (E.15) we apply the Pochhammer shift identity (E.12) twice. 
Now we are in the position to derive the formula for the angular single massive integral

$$
\begin{aligned}
& I_{j, l}\left(v_{12}, v_{11}, 0, \varepsilon\right)=\frac{2 \pi}{1-2 \varepsilon} v_{12}^{1-j-l-\varepsilon} \\
& \quad \times F_{B}^{(3)}\left(\frac{j}{2}, \frac{l}{2}, \frac{3-j-l-2 \varepsilon}{2}, \frac{j+1}{2}, \frac{l+1}{2}, \frac{2-j-l-2 \varepsilon}{2}, \frac{3}{2}-\varepsilon, 1-\frac{v_{11}}{v_{12}}, 1,1-v_{12}\right) \\
& =\frac{2 \pi}{1-2 \varepsilon} v_{12}^{1-j-l-\varepsilon} \sum_{m, n, p=0}^{\infty} \frac{(j)_{2 m}(l)_{2 n}(2-j-l-2 \varepsilon)_{2 p}}{\left(\frac{3}{2}-\varepsilon\right)_{m+n+p} m ! n ! p !} 4^{-m-n-p}\left(1-\frac{v_{11}}{v_{12}}\right)^{m}\left(1-v_{12}\right)^{p} \\
& =\frac{2 \pi}{1-2 \varepsilon} v_{12}^{1-j-l-\varepsilon}{ }_{2} F_{1}\left(\frac{l}{2}, \frac{l+1}{2}, \frac{3}{2}-\varepsilon, 1\right) \\
& \quad \times \sum_{m, p=0}^{\infty} \frac{(j)_{2 m}(2-j-l-2 \varepsilon)_{2 p}(1-l-\varepsilon)_{m+p}}{(2-l-2 \varepsilon)_{2 m+2 p} m ! p !}\left(1-\frac{v_{11}}{v_{12}}\right)^{m}\left(1-v_{12}\right)^{p} \\
& \stackrel{(E .7)}{=} I_{l}^{(0)}(\varepsilon) v_{12}^{-j} F_{1}\left(j, 1-l-\varepsilon, 1-l-\varepsilon, 2-l-2 \varepsilon, 1-\frac{1+\sqrt{1-v_{11}}}{v_{12}}, 1-\frac{1-\sqrt{1-v_{11}}}{v_{12}}\right) \\
& =I_{j, l}^{(1)}\left(v_{12}, v_{11}, \varepsilon\right),
\end{aligned}
$$

which is in the exact agreement with the known analytic result for the single massive integral in $D$ dimensions [48].

\section{E.3 Massless integral}

We perform the check of the massless integral starting from the single massive result (E.16), where we put $v_{11}=0$. Using the identities

$$
\begin{aligned}
F_{1}\left(a, b_{1}, b_{2}, c, x, 1\right) & ={ }_{2} F_{1}\left(a, b_{2}, c, 1\right){ }_{2} F_{1}\left(a, b_{1}, c-b_{2}, x\right), \\
{ }_{2} F_{1}(a, b, c, x) & =(1-x)^{-a}{ }_{2} F_{1}\left(a, c-b, c, \frac{x}{x-1}\right),
\end{aligned}
$$

the Gauss theorem (3.37)

$$
\begin{aligned}
{ }_{2} F_{1}(j, 1-l-\varepsilon, 2-l-2 \varepsilon, 1) & =\frac{\Gamma(2-l-2 \varepsilon) \Gamma(1-j-\varepsilon)}{\Gamma(2-l-j-2 \varepsilon) \Gamma(1-\varepsilon)}=\frac{(2-j-l-2 \varepsilon)_{j}}{(1-j-\varepsilon)_{j}} \\
& =\frac{(2-j-l-2 \varepsilon)_{j+l}}{(1-j-\varepsilon)_{j}(2-l-2 \varepsilon)_{l}},
\end{aligned}
$$

and eq. (3.43) we obtain the formula

$$
\begin{aligned}
I_{j, l}\left(v_{12}, 0,0 ; \varepsilon\right) & =I_{l}^{(0)}(\varepsilon) v_{12}^{-j} F_{1}\left(j, 1-l-\varepsilon, 1-l-\varepsilon, 2-l-2 \varepsilon, 1-\frac{2}{v_{12}}, 1\right) \\
& =I_{l}^{(0)}(\varepsilon) v_{12}^{-j}{ }_{2} F_{1}(j, 1-l-\varepsilon, 2-l-2 \varepsilon, 1){ }_{2} F_{1}\left(j, 1-l-\varepsilon, 1-\varepsilon, 1-\frac{2}{v_{12}}\right) \\
& =\frac{I_{l}^{(0)}(\varepsilon)}{2^{j}}{ }_{2} F_{1}(j, 1-l-\varepsilon, 2-l-2 \varepsilon, 1)_{2} F_{1}\left(j, l, 1-\varepsilon, 1-\frac{v_{12}}{2}\right) \\
& =\frac{I^{(0)}(\varepsilon)}{2^{j+l}} \frac{(2-j-l-2 \varepsilon)_{j+l}}{(1-j-\varepsilon)_{j}(1-l-\varepsilon)_{l}}{ }_{2} F_{1}\left(j, l, 1-\varepsilon, 1-\frac{v_{12}}{2}\right) \\
& =I_{j, l}^{(0)}\left(v_{12} ; \varepsilon\right),
\end{aligned}
$$

which is again in complete agreement with the result of ref. [48]. 


\section{F Double Nielsen polylogarithms}

\section{F.1 Basic properties of double Nielsen polylogarithms}

Basic properties of the double Nielsen polylogarithm

$$
S_{n, p_{1}, p_{2}}(x, y) \equiv \frac{(-1)^{n+p_{1}+p_{2}-1}}{(n-1) ! p_{1} ! p_{2} !} \int_{0}^{1} \frac{\mathrm{d} t}{t} \log ^{n-1} t \log ^{p_{1}}(1-x t) \log ^{p_{2}}(1-y t),
$$

are:

(1) symmetry $S_{n, p_{1}, p_{2}}(x, y)=S_{n, p_{2}, p_{1}}(y, x)$,

(2) reduction to single Nielsen polylogarithm $S_{n, p_{1}, 0}(x, y)=S_{n, p_{1}}(x)$ and $S_{n, 0, p_{2}}(x, y)=$ $S_{n, p_{2}}(y)$.

With expansion

$$
\log ^{m}(1+x)=m ! \sum_{k=m}^{\infty} S_{k}^{(m)} \frac{x^{k}}{k !} \quad \text { for }|x|<1
$$

where

$$
S_{k}^{(m)}=\sum_{i=0}^{k-m} \frac{1}{i !}\left(\begin{array}{c}
k-1+i \\
k-m+i
\end{array}\right)\left(\begin{array}{c}
2 k-m \\
k-m-i
\end{array}\right) \sum_{j=0}^{i}(-1)^{i}\left(\begin{array}{l}
i \\
j
\end{array}\right)^{k-m+i},
$$

are Stirling numbers of the 1st kind and integral

$$
\int_{0}^{1} \mathrm{~d} t t^{m-1}(\log t)^{n-1}=-(-m)^{-n}(n-1) !
$$

one obtains the series

$$
S_{n, p_{1}, p_{2}}(x, y)=\sum_{k, l=0}^{\infty} \frac{(-1)^{k+l} S_{p_{1}+k}^{\left(p_{1}\right)} S_{p_{2}+l}^{\left(p_{2}\right)}}{\left(p_{1}+k\right) !\left(p_{2}+l\right) !\left(p_{1}+p_{2}+k+l\right)^{n}} x^{p_{1}+k} y^{p_{2}+l} \quad \text { for }|x| \leq 1,|y| \leq 1 .
$$

\section{F.2 Relation of Goncharov and Nielsen polylogarithms}

Goncharov polylogarithms are a powerful tool for working with a wide range of generalized logarithms [87]. They are recursively defined via the iterated integral

$$
G\left(a_{1}, \ldots, a_{n} ; z\right) \equiv \int_{0}^{1} \frac{\mathrm{d} t}{t-a_{1}} G\left(a_{2}, \ldots, a_{n} ; t\right)
$$

with $G(z)=1$. It holds with $\vec{a}_{n}=(\underbrace{a, \ldots, a}_{n \text {-times }})$ :

$$
\begin{aligned}
G\left(\overrightarrow{0}_{n} ; z\right) & =\frac{1}{n !} \log ^{n} z, & G\left(\vec{a}_{n} ; z\right) & =\frac{1}{n !} \log ^{n}\left(1-\frac{z}{a}\right), \\
G\left(\overrightarrow{0}_{n-1}, a ; z\right) & =-\operatorname{Li}_{n}\left(\frac{z}{a}\right), & G\left(\overrightarrow{0}_{n-k}, \vec{a}_{k} ; z\right) & =(-1)^{k} S_{n-k, k}\left(\frac{z}{a}\right) .
\end{aligned}
$$


Goncharov polylogarithms are related to other polylogarithms by the relations:

(1) with Nielsen polylogarithms by $S_{n, p}(z)=(-1)^{p} G(\overbrace{0, \ldots, 0}^{n}, \overbrace{1, \ldots, 1}^{p} ; z)$;

(2) with Harmonic polylogarithms by $H\left(a_{1}, \ldots a_{n} ; z\right)=(-1)^{p} G\left(a_{1}, \ldots, a_{n} ; z\right)$, where $a_{i} \in\{0,1\}$

(3) with Multiple polylogarithms by

$$
G\left(\overrightarrow{0}_{m_{1}-1}, a_{1}, \ldots, \overrightarrow{0}_{m_{k}-1}, a_{k} ; z\right)=(-1)^{k} \operatorname{Li}_{m_{k}, \ldots, m_{1}}\left(\frac{a_{k-1}}{a_{k}}, \ldots, \frac{a_{1}}{a_{2}}, \frac{z}{a_{1}}\right)
$$

where

$$
\operatorname{Li}_{m_{1}, \ldots, m_{k}}=\sum_{0<n_{1}<\cdots<n_{k}} \frac{z_{1}^{n_{1}} z_{2}^{n_{2}} \ldots z_{k}^{n_{k}}}{n_{1}^{m_{1}} n_{2}^{m_{2}} \ldots n_{k}^{m_{k}}}
$$

Goncharov polylogarithms satisfy the shuffle algebra

$$
G(\vec{a} ; z) G(\vec{b}, z)=\sum_{\vec{c} \in \vec{a} \sqcup \vec{b}} G(\vec{c} ; z),
$$

where $\vec{a} \uplus \vec{b}$ denotes the set of all shuffles of the vectors $\vec{a}$ and $\vec{b}$, i.e. all the ways of interlacing them while keeping the order within $\vec{a}$ and $\vec{b}$. Goncharov polylogarithm has the integration by parts property

$$
\begin{aligned}
G\left(z_{1}, z_{2}, \ldots, z_{n} ; 1\right)= & G\left(z_{1} ; 1\right) G\left(z_{2}, \ldots, z_{n} ; 1\right)-G\left(z_{2}, z_{1} ; 1\right) G\left(z_{3}, \ldots, z_{n} ; 1\right) \\
& +\cdots-(-1)^{n} G\left(z_{n}, \ldots, z_{2}, z_{1} ; 1\right)
\end{aligned}
$$

Another useful identity is the logarithmic integral

$$
G\left(\overrightarrow{0}_{m}, z_{1}, z_{2}, \ldots, z_{n} ; 1\right)=\frac{(-1)^{m}}{m !} \int_{0}^{1} \frac{\mathrm{d} t \log ^{m} t}{t-z_{1}} G\left(z_{2}, \ldots, z_{n} ; t\right) .
$$

\section{F.3 Double Nielsen polylogarithms in terms of multiple polylogarithms}

Using the logarithmic integral eq. (F.11) and the shuffle algebra we can represent the double Nielsen polylogarithm in terms of multiple polylogarithms. Starting from eq. (F.1) we find

$$
\begin{aligned}
S_{n, p_{1}, p_{2}}(x, y) & =\frac{(-1)^{n+p_{1}+p_{2}-1}}{(n-1) ! p_{1} ! p_{2} !} \int_{0}^{1} \frac{\mathrm{d} t}{t} \log ^{n-1} t \log ^{p_{1}}(1-x t) \log ^{p_{2}}(1-y t) \\
& =\frac{(-1)^{n+p_{1}+p_{2}-1}}{(n-1) !} \int_{0}^{1} \frac{\mathrm{d} t}{t} \log ^{n-1} t G\left(\left(\frac{\overrightarrow{1}}{x}\right)_{p_{1}} ; t\right) G\left(\left(\frac{\overrightarrow{1}}{y}\right)_{p_{2}} ; t\right) \\
& =\frac{(-1)^{n+p_{1}+p_{2}-1}}{(n-1) !} \int_{0}^{1} \frac{\mathrm{d} t}{t} \log ^{n-1} t \sum_{\vec{z} \in \frac{1}{x} \sqcup \frac{\overrightarrow{1}}{y}} G\left(z_{1}, \ldots, z_{p_{1}+p_{2}} ; t\right) \\
& =(-1)^{p_{1}+p_{2}} \sum_{\vec{z} \in \frac{\overrightarrow{1}}{x} \sqcup \frac{\overrightarrow{1}}{y}} G\left(\overrightarrow{0}_{n-1}, 0, z_{1}, \ldots, z_{p_{1}+p_{2}} ; 1\right) \\
& =\sum_{\vec{z} \in \vec{x}_{p_{1}} \sqcup \vec{y}_{p_{2}}} \operatorname{Li}_{1,1, \ldots, 1, n+1}\left(\frac{z_{p_{1}+p_{2}}}{z_{p_{1}+p_{2}-1}}, \ldots, \frac{z_{2}}{z_{1}}, z_{1}\right) .
\end{aligned}
$$


Therefore, double Nielsen polylogarithms can be calculated from multiple polylogarithms using the identity

$$
S_{n, p_{1}, p_{2}}(x, y)=\sum_{\vec{z} \in \vec{x}_{p_{1}} \amalg \vec{y}_{p_{2}}} \operatorname{Li}_{1,1, \ldots, 1, n+1}\left(\frac{z_{p_{1}+p_{2}}}{z_{p_{1}+p_{2}-1}}, \ldots, \frac{z_{2}}{z_{1}}, z_{1}\right) .
$$

For the reduction and calculation of multiple polylogarithms there exist publicly available tools $[88,89]$. Some explicit examples for the shuffle sums are

$$
\begin{aligned}
S_{n, 1,1}(x, y)= & \sum_{\vec{z} \in x \amalg y} \operatorname{Li}_{1, n+1}\left(\frac{z_{2}}{z_{1}}, z_{1}\right)=\operatorname{Li}_{1, n+1}\left(\frac{y}{x}, x\right)+\operatorname{Li}_{1, n+1}\left(\frac{x}{y}, y\right), \\
S_{n, 2,1}(x, y)= & \sum_{\vec{z} \in \vec{x}_{2} \sqcup y} \operatorname{Li}_{1,1, n+1}\left(\frac{z_{3}}{z_{2}}, \frac{z_{2}}{z_{1}}, z_{1}\right) \\
= & \operatorname{Li}_{1,1, n+1}\left(\frac{y}{x}, 1, x\right)+\operatorname{Li}_{1,1, n+1}\left(\frac{x}{y}, \frac{y}{x}, x\right)+\operatorname{Li}_{1,1, n+1}\left(1, \frac{x}{y}, y\right), \\
S_{n, 2,2}(x, y)= & \sum_{\vec{z} \in \vec{x}_{2} \sqcup \vec{y}_{2}} \operatorname{Li}_{1,1,1, n+1}\left(\frac{z_{4}}{z_{3}}, \frac{z_{3}}{z_{2}}, \frac{z_{2}}{z_{1}}, z_{1}\right) \\
= & \operatorname{Li}_{1,1,1, n+1}\left(1, \frac{y}{x}, 1, x\right)+\operatorname{Li}_{1,1,1, n+1}\left(\frac{y}{x}, \frac{x}{y}, \frac{y}{x}, x\right) \\
& +\operatorname{Li}_{1,1,1, n+1}\left(\frac{x}{y}, 1, \frac{y}{x}, 1, x\right)+\operatorname{Li}_{1,1,1, n+1}\left(\frac{y}{x}, 1, \frac{x}{y}, y\right) \\
& +\operatorname{Li}_{1,1,1, n+1}\left(\frac{x}{y}, \frac{y}{x}, \frac{x}{y}, y\right)+\operatorname{Li}_{1,1,1, n+1}\left(1, \frac{x}{y}, 1, y\right)
\end{aligned}
$$

\section{G Tables of angular integrals}

\section{G.1 Massless integral with one denominator}

$$
\begin{aligned}
I_{-4}^{(0)}(\varepsilon) & =4 I^{(0)}(\varepsilon) \frac{(3-\varepsilon)(4-\varepsilon)}{(3-2 \varepsilon)(5-2 \varepsilon)} \\
& =\frac{32 \pi}{5}+\frac{1192 \pi \varepsilon}{75}+\frac{38152 \pi \varepsilon^{2}}{1125}+\frac{1168912 \pi \varepsilon^{3}}{16875}+\frac{35313472 \pi \varepsilon^{4}}{253125}+\mathcal{O}\left(\varepsilon^{5}\right) \\
I_{-3}^{(0)}(\varepsilon) & =2 I^{(0)}(\varepsilon) \frac{3-\varepsilon}{3-2 \varepsilon} \\
& =4 \pi+\frac{28 \pi \varepsilon}{3}+\frac{176 \pi \varepsilon^{2}}{9}+\frac{1072 \pi \varepsilon^{3}}{27}+\frac{6464 \pi \varepsilon^{4}}{81}+\mathcal{O}\left(\varepsilon^{5}\right) \\
I_{-2}^{(0)}(\varepsilon) & =2 I^{(0)}(\varepsilon) \frac{2-\varepsilon}{3-2 \varepsilon} \\
& =\frac{8 \pi}{3}+\frac{52 \pi \varepsilon}{9}+\frac{320 \pi \varepsilon^{2}}{27}+\frac{1936 \pi \varepsilon^{3}}{81}+\frac{11648 \pi \varepsilon^{4}}{243}+\mathcal{O}\left(\varepsilon^{5}\right) \\
I_{0}^{(0)}(\varepsilon) & =I_{-1}^{(0)}(\varepsilon)=I^{(0)}(\varepsilon)=\frac{2 \pi}{1-2 \varepsilon}=2 \pi+4 \pi \varepsilon+8 \pi \varepsilon^{2}+16 \pi \varepsilon^{3}+32 \pi \varepsilon^{4}+\mathcal{O}\left(\varepsilon^{5}\right), \\
I_{1}^{(0)}(\varepsilon) & =-I^{(0)}(\varepsilon) \frac{1-2 \varepsilon}{2 \varepsilon}=-\frac{\pi}{\varepsilon}, \\
I_{2}^{(0)}(\varepsilon) & =-I^{(0)}(\varepsilon) \frac{1-2 \varepsilon}{2(1+\varepsilon)}=-\frac{\pi}{1+\varepsilon}=-\pi+\pi \varepsilon-\pi \varepsilon^{2}+\pi \varepsilon^{3}-\pi \varepsilon^{4}+\mathcal{O}\left(\varepsilon^{5}\right) .
\end{aligned}
$$




\section{G.2 Massive integral with one denominator}

$$
\begin{aligned}
I_{-4}^{(1)}\left(v_{11} ; \varepsilon\right)= & I^{(0)}(\varepsilon) \frac{2\left(6-3 v_{11}-2 \varepsilon\right)(4-\varepsilon)-3 v_{11}\left(4-v_{11}-2 \varepsilon\right)}{(3-2 \varepsilon)(5-2 \varepsilon)} \\
= & \frac{2}{5} \pi\left(16-12 v_{11}+v_{11}^{2}\right)+\frac{4}{75} \pi\left(298-246 v_{11}+23 v_{11}^{2}\right) \varepsilon+\mathcal{O}\left(\varepsilon^{2}\right), \\
I_{-3}^{(1)}\left(v_{11} ; \varepsilon\right)= & I^{(0)}(\varepsilon) \frac{6-3 v_{11}-2 \varepsilon}{3-2 \varepsilon} \\
= & 2 \pi\left(2-v_{11}\right)+\frac{4}{3} \pi\left(7-4 v_{11}\right) \varepsilon+\mathcal{O}\left(\varepsilon^{2}\right) \\
I_{-2}^{(1)}\left(v_{11} ; \varepsilon\right)= & I^{(0)}(\varepsilon) \frac{4-v_{11}-2 \varepsilon}{3-2 \varepsilon} \\
= & \frac{2}{3} \pi\left(4-v_{11}\right)+\frac{4}{9} \pi\left(13-4 v_{11}\right) \varepsilon+\mathcal{O}\left(\varepsilon^{2}\right), \\
I_{0}^{(1)}\left(v_{11} ; \varepsilon\right)= & I_{-1}^{(1)}\left(v_{11} ; \varepsilon\right)=I^{(0)}(\varepsilon)=\frac{2 \pi}{1-2 \varepsilon}=2 \pi+4 \pi \varepsilon+\mathcal{O}\left(\varepsilon^{2}\right) \\
I_{1}^{(1)}\left(v_{11} ; \varepsilon\right)= & \frac{\pi \log \left(\frac{1+\sqrt{1-v_{11}}}{1-\sqrt{1-v_{11}}}\right)}{\sqrt{1-v_{11}}}-\frac{\pi \varepsilon\left(\log ^{2}\left(\frac{1+\sqrt{1-v_{11}}}{1-\sqrt{1-v_{11}}}\right)+4 \operatorname{Li}_{2}\left(\frac{2 \sqrt{1-v_{11}}}{\sqrt{1-v_{11}}-1}\right)\right)}{2 \sqrt{1-v_{11}}} \\
& +\mathcal{O}\left(\varepsilon^{2}\right) \\
& \frac{2 \varepsilon}{v_{11}} I_{1}^{(1)}\left(v_{11} ; \varepsilon\right)+\frac{1-2 \varepsilon}{v_{11}} I^{(0)}=\frac{2 \pi}{v_{11}}\left[\begin{array}{c}
\varepsilon \log \left(\frac{1+\sqrt{1-v_{11}}}{1-\sqrt{1-v_{11}}}\right) \\
\sqrt{1-v_{11}}
\end{array}\right] \\
& +\mathcal{O}\left(\varepsilon^{2}\right) .
\end{aligned}
$$

\section{G.3 Massless integral with two denominators}

$$
\begin{aligned}
I_{-4,0}^{(0)}\left(v_{12} ; \varepsilon\right)= & I_{-4}^{(0)}(\varepsilon) \\
I_{-4,-1}^{(0)}\left(v_{12} ; \varepsilon\right)= & \frac{1}{4(3-\varepsilon)}\left[(7-2 \varepsilon) I_{-4}^{(0)}(\varepsilon)+(13-2 \varepsilon) I_{-3,-1}^{(0)}\left(v_{12} ; \varepsilon\right)-5 v_{12} I_{-3}^{(0)}(\varepsilon)\right] \\
= & \frac{32 \pi}{15}\left(5-2 v_{12}\right)+\frac{8 \pi}{225}\left(785-338 v_{12}\right) \varepsilon+\mathcal{O}\left(\varepsilon^{2}\right) \\
I_{-4,-2}^{(0)}\left(v_{12} ; \varepsilon\right)= & \frac{1}{7-2 \varepsilon}\left[(5-\varepsilon) I_{-4,-1}^{(0)}\left(v_{12} ; \varepsilon\right)+(7-\varepsilon) I_{-3,-2}^{(0)}\left(v_{12} ; \varepsilon\right)-3 v_{12} I_{-3,-1}^{(0)}\left(v_{12} ; \varepsilon\right)\right] \\
= & \frac{64 \pi}{105}\left(30-20 v_{12}+3 v_{12}^{2}\right)+\frac{16 \pi}{11025}\left(34470-24380 v_{12}+3909 v_{12}^{2}\right) \varepsilon \\
& +\mathcal{O}\left(\varepsilon^{2}\right) \\
I_{-4,-3}^{(0)}\left(v_{12} ; \varepsilon\right)= & \frac{1}{4(4-\varepsilon)}\left[(13-2 \varepsilon) I_{-4,-2}^{(0)}\left(v_{12} ; \varepsilon\right)+(15-2 \varepsilon) I_{-3,-3}^{(0)}\left(v_{12} ; \varepsilon\right)\right. \\
& \left.-7 v_{12} I_{-3,-2}^{(0)}\left(v_{12} ; \varepsilon\right)\right]=\frac{16 \pi}{35}\left(70-60 v_{12}+15 v_{12}^{2}-v_{12}^{3}\right) \\
& +\frac{16 \pi}{3675}\left(20895-18810 v_{12}+4965 v_{12}^{2}-352 v_{12}^{3}\right) \varepsilon+\mathcal{O}\left(\varepsilon^{2}\right)
\end{aligned}
$$




$$
\begin{aligned}
& I_{-4,-4}^{(0)}\left(v_{12} ; \varepsilon\right)=\frac{1}{9-\varepsilon}\left[(8-\varepsilon)\left(I_{-4,-3}^{(0)}\left(v_{12} ; \varepsilon\right)+I_{-3,-4}^{(0)}\left(v_{12} ; \varepsilon\right)\right)-4 v_{12} I_{-3,-3}^{(0)}\left(v_{12} ; \varepsilon\right)\right] \\
& =\frac{16 \pi}{315}\left(1120-1120 v_{12}+360 v_{12}^{2}-40 v_{12}^{3}+v_{12}^{4}\right) \\
& +\frac{32 \pi}{99225}\left(518630-540680 v_{12}+181890 v_{12}^{2}-21260 v_{12}^{3}+563 v_{12}^{4}\right) \varepsilon \\
& +\mathcal{O}\left(\varepsilon^{2}\right), \\
& I_{-4,1}^{(0)}\left(v_{12} ; \varepsilon\right)=\left(1-v_{12}\right) I_{-3}^{(0)}(\varepsilon) \\
& +\frac{v_{12}}{2(2-\varepsilon)}\left[3 I_{-2}^{(0)}(\varepsilon)+(7-2 \varepsilon) I_{-3,1}^{(0)}\left(v_{12} ; \varepsilon\right)-3 v_{12} I_{-2,1}^{(0)}\left(v_{12} ; \varepsilon\right)\right] \\
& =-\frac{\pi}{\varepsilon} v_{12}^{4}+\frac{\pi}{6}\left(24+16 v_{12}+12 v_{12}^{2}+12 v_{12}^{3}-25 v_{12}^{4}\right) \\
& +\frac{\pi}{36}\left(336+256 v_{12}+228 v_{12}^{2}+300 v_{12}^{3}-415 v_{12}^{4}\right) \varepsilon+\mathcal{O}\left(\varepsilon^{2}\right), \\
& I_{-4,2}^{(0)}\left(v_{12} ; \varepsilon\right)=\left(1-v_{12}\right) I_{-3,1}^{(0)}\left(v_{12} ; \varepsilon\right) \\
& +\frac{v_{12}}{3-2 \varepsilon}\left[3 I_{-2,1}^{(0)}\left(v_{12} ; \varepsilon\right)+(7-2 \varepsilon) I_{-3,2}^{(0)}\left(v_{12} ; \varepsilon\right)-3 v_{12} I_{-2,2}^{(0)}\left(v_{12} ; \varepsilon\right)\right] \\
& =-\frac{2 \pi}{\varepsilon}\left(8-5 v_{12}\right) v_{12}^{3}+\frac{4 \pi}{3}\left(2+4 v_{12}+9 v_{12}^{2}-44 v_{12}^{3}+20 v_{12}^{4}\right) \\
& +\frac{2 \pi}{9}\left(26+64 v_{12}+198 v_{12}^{2}-680 v_{12}^{3}+305 v_{12}^{4}\right) \varepsilon+\mathcal{O}\left(\varepsilon^{2}\right), \\
& I_{-3,0}^{(0)}\left(v_{12} ; \varepsilon\right)=I_{-3}^{(0)}(\varepsilon), \\
& I_{-3,-1}^{(0)}\left(v_{12} ; \varepsilon\right)=\frac{1}{(5-2 \varepsilon)}\left[(3-\varepsilon) I_{-3}^{(0)}(\varepsilon)+(5-\varepsilon) I_{-2,-1}^{(0)}\left(v_{12} ; \varepsilon\right)-2 v_{12} I_{-2}^{(0)}(\varepsilon)\right] \\
& =\frac{4 \pi}{5}\left(8-3 v_{12}\right)+\frac{4 \pi}{75}\left(298-123 v_{12}\right) \varepsilon+\mathcal{O}\left(\varepsilon^{2}\right), \\
& I_{-3,-2}^{(0)}\left(v_{12} ; \varepsilon\right)=\frac{1}{4(3-\varepsilon)}\left[(9-2 \varepsilon) I_{-3,-1}^{(0)}\left(v_{12} ; \varepsilon\right)+(11-2 \varepsilon) I_{-2,-2}^{(0)}\left(v_{12} ; \varepsilon\right)\right. \\
& \left.-5 v_{12} I_{-2,-1}^{(0)}\left(v_{12} ; \varepsilon\right)\right] \\
& =\frac{4 \pi}{15}\left(40-24 v_{12}+3 v_{12}^{2}\right)+\frac{8 \pi}{225}\left(785-507 v_{12}+69 v_{12}^{2}\right) \varepsilon+\mathcal{O}\left(\varepsilon^{2}\right) \text {, } \\
& I_{-3,-3}^{(0)}\left(v_{12} ; \varepsilon\right)=\frac{1}{7-2 \varepsilon}\left[(6-\varepsilon)\left(I_{-3,-2}^{(0)}\left(v_{12} ; \varepsilon\right)+I_{-2,-3}^{(0)}\left(v_{12} ; \varepsilon\right)\right)-3 v_{12} I_{-2,-2}^{(0)}\left(v_{12} ; \varepsilon\right)\right] \\
& =\frac{4 \pi}{35}\left(160-120 v_{12}+24 v_{12}^{2}-v_{12}^{3}\right) \\
& +\frac{8 \pi}{3675}\left(22980-18285 v_{12}+3909 v_{12}^{2}-176 v_{12}^{3}\right) \varepsilon+\mathcal{O}\left(\varepsilon^{2}\right), \\
& I_{-3,1}^{(0)}\left(v_{12} ; \varepsilon\right)=\left(1-v_{12}\right) I_{-2}^{(0)}(\varepsilon) \\
& +\frac{v_{12}}{(3-2 \varepsilon)}\left[2 I_{-1}^{(0)}(\varepsilon)+(5-2 \varepsilon) I_{-2,1}^{(0)}\left(v_{12} ; \varepsilon\right)-2 v_{12} I_{-1,1}^{(0)}\left(v_{12} ; \varepsilon\right)\right] \\
& =-\frac{\pi}{\varepsilon} v_{12}^{3}+\frac{\pi}{3}\left(8+6 v_{12}+6 v_{12}^{2}-11 v_{12}^{3}\right) \\
& +\frac{\pi}{9}\left(52+48 v_{12}+66 v_{12}^{2}-85 v_{12}^{3}\right) \varepsilon+\mathcal{O}\left(\varepsilon^{2}\right) \text {, }
\end{aligned}
$$




$$
\begin{aligned}
& I_{-3,2}^{(0)}\left(v_{12} ; \varepsilon\right)=\left(1-v_{12}\right) I_{-2,1}^{(0)}\left(v_{12} ; \varepsilon\right) \\
& +\frac{v_{12}}{(1-\varepsilon)}\left[I_{-1,1}^{(0)}\left(v_{12} ; \varepsilon\right)+(2-\varepsilon) I_{-2,2}^{(0)}\left(v_{12} ; \varepsilon\right)-v_{12} I_{-1,2}^{(0)}\left(v_{12} ; \varepsilon\right)\right] \\
& =-\frac{3 \pi}{\varepsilon}\left(3-2 v_{12}\right) v_{12}^{2}+\pi\left(2+6 v_{12}-27 v_{12}^{2}+12 v_{12}^{3}\right) \\
& +\pi\left(4+18 v_{12}-63 v_{12}^{2}+30 v_{12}^{3}\right) \varepsilon+\mathcal{O}\left(\varepsilon^{2}\right), \\
& I_{-2,0}^{(0)}\left(v_{12} ; \varepsilon\right)=I_{-2}^{(0)}(\varepsilon), \\
& I_{-2,-1}^{(0)}\left(v_{12} ; \varepsilon\right)=\frac{1}{4(2-\varepsilon)}\left[(7-2 \varepsilon) I_{-2}^{(0)}(\varepsilon)+(5-2 \varepsilon) I_{-1,-1}^{(0)}\left(v_{12} ; \varepsilon\right)-3 v_{12} I^{(0)}(\varepsilon)\right] \\
& =\frac{4 \pi}{3}\left(3-v_{12}\right)+\frac{4 \pi}{9}\left(21-8 v_{12}\right) \varepsilon+\mathcal{O}\left(\varepsilon^{2}\right), \\
& I_{-2,-2}^{(0)}\left(v_{12} ; \varepsilon\right)=\frac{1}{5-2 \varepsilon}\left[(4-\varepsilon)\left(I_{-2,-1}^{(0)}\left(v_{12} ; \varepsilon\right)+I_{-1,-2}^{(0)}\left(v_{12} ; \varepsilon\right)\right)-2 v_{12} I_{-1,-1}^{(0)}\left(v_{12} ; \varepsilon\right)\right] \\
& =\frac{4 \pi}{15}\left(24-12 v_{12}+v_{12}^{2}\right)+\frac{8 \pi}{225}\left(447-246 v_{12}+23 v_{12}^{2}\right) \varepsilon+\mathcal{O}\left(\varepsilon^{2}\right), \\
& I_{-2,1}^{(0)}\left(v_{12} ; \varepsilon\right)=\left(1-v_{12}\right) I^{(0)}(\varepsilon) \\
& +\frac{v_{12}}{2(1-\varepsilon)}\left[I^{(0)}(\varepsilon)+(3-2 \varepsilon) I_{-1,1}^{(0)}\left(v_{12} ; \varepsilon\right)-v_{12} I_{1}^{(0)}(\varepsilon)\right] \\
& =-\frac{\pi}{\varepsilon} v_{12}^{2}+\pi\left(2+2 v_{12}-3 v_{12}^{2}\right)+\pi\left(4+6 v_{12}-7 v_{12}^{2}\right) \varepsilon+\mathcal{O}\left(\varepsilon^{2}\right), \\
& I_{-2,2}^{(0)}\left(v_{12} ; \varepsilon\right)=\left(1-v_{12}\right) I_{-1,1}^{(0)}\left(v_{12} ; \varepsilon\right) \\
& +\frac{v_{12}}{(1-2 \varepsilon)}\left[I_{1}^{(0)}(\varepsilon)+2(1-\varepsilon) I_{-1,2}^{(0)}\left(v_{12} ; \varepsilon\right)-v_{12} I_{2}^{(0)}(\varepsilon)\right] \\
& =-\frac{\pi}{\varepsilon} v_{12}\left(4-3 v_{12}\right)+\pi\left(2-8 v_{12}+3 v_{12}^{2}\right)+\pi\left(4-16 v_{12}+9 v_{12}^{2}\right) \varepsilon \\
& +\mathcal{O}\left(\varepsilon^{2}\right), \\
& I_{-1,0}^{(0)}\left(v_{12} ; \varepsilon\right)=I^{(0)}(\varepsilon), \\
& I_{-1,-1}^{(0)}\left(v_{12} ; \varepsilon\right)=\frac{4-v_{12}-2 \varepsilon}{3-2 \varepsilon} I^{(0)} \\
& =\frac{2 \pi}{3}\left(4-v_{12}\right)+\frac{4 \pi}{9}\left(13-4 v_{12}\right) \varepsilon+\mathcal{O}\left(\varepsilon^{2}\right), \\
& I_{-1,1}^{(0)}\left(v_{12} ; \varepsilon\right)=\left(1-v_{12}\right) I^{(0)}(\varepsilon)+v_{12} I_{1}^{(0)}(\varepsilon) \\
& =-\frac{\pi}{\varepsilon} v_{12}+2 \pi\left(1-v_{12}\right)+4 \pi\left(1-v_{12}\right) \varepsilon+\mathcal{O}\left(\varepsilon^{2}\right), \\
& I_{-1,2}^{(0)}\left(v_{12} ; \varepsilon\right)=\left(1-v_{12}\right) I_{1}^{(0)}(\varepsilon)+v_{12} I_{2}^{(0)}(\varepsilon) \\
& =-\frac{\pi}{\varepsilon}\left(1-v_{12}\right)-\pi v_{12}+\pi v_{12} \varepsilon+\mathcal{O}\left(\varepsilon^{2}\right), \\
& I_{1,1}^{(0)}\left(v_{12} ; \varepsilon\right)=\frac{2 \pi}{v_{12}}\left[-\frac{1}{\varepsilon}+\log \left(\frac{v_{12}}{2}\right)-\varepsilon\left(\frac{1}{2} \log ^{2}\left(\frac{v_{12}}{2}\right)+\operatorname{Li}_{2}\left(1-\frac{v_{12}}{2}\right)\right)\right] \\
& +\mathcal{O}\left(\varepsilon^{2}\right) \text {, }
\end{aligned}
$$




$$
\begin{aligned}
I_{1,2}^{(0)}\left(v_{12} ; \varepsilon\right)= & \frac{1}{v_{12}}\left[(1+2 \varepsilon) I_{1,1}^{(0)}\left(v_{12} ; \varepsilon\right)-2 \varepsilon I_{1}^{(0)}(\varepsilon)+I_{2}^{(0)}(\varepsilon)\right] \\
= & \frac{\pi}{v_{12}^{2}}\left[-\frac{2}{\varepsilon}-4+v_{12}+2 \log \left(\frac{v_{12}}{2}\right)\right. \\
& \left.\left.+\varepsilon\left(v_{12}+4 \log \left(\frac{v_{12}}{2}\right)-\log ^{2}\left(\frac{v_{12}}{2}\right)-2 \operatorname{Li}_{2}\left(1-\frac{v_{12}}{2}\right)\right)\right]+\mathcal{O}\left(\varepsilon^{2}\right), \quad \text { G. } 36\right) \\
I_{2,2}^{(0)}\left(v_{12} ; \varepsilon\right)= & \frac{1}{v_{12}^{2}}\left[(1+2 \varepsilon)\left(1-v_{12}\right) I_{1,1}^{(0)}\left(v_{12} ; \varepsilon\right)-(1+2 \varepsilon) I_{2}^{(0)}(\varepsilon)+(3+2 \varepsilon) v_{12} I_{1,2}^{(0)}\left(v_{12} ; \varepsilon\right)\right] \\
= & -\frac{2 \pi}{v_{12}^{3}}\left[\frac{4-v_{12}}{\varepsilon}+10-4 v_{12}-\left(4-v_{12}\right) \log \left(\frac{v_{12}}{2}\right)\right. \\
& +\varepsilon\left(4-3 v_{12}-2\left(5-v_{12}\right) \log \left(\frac{v_{12}}{2}\right)+\left(2-\frac{v_{12}}{2}\right) \log ^{2}\left(\frac{v_{12}}{2}\right)\right. \\
& \left.\left.+\left(4-v_{12}\right) \operatorname{Li}_{2}\left(1-\frac{v_{12}}{2}\right)\right)\right]+\mathcal{O}\left(\varepsilon^{2}\right) .
\end{aligned}
$$

\section{G.4 Single massive integral with two denominators}

$$
\begin{aligned}
& I_{-4,-1}^{(1)}\left(v_{12}, v_{11} ; \varepsilon\right)= \frac{1}{4(3-\varepsilon)}\left[(7-2 \varepsilon) I_{-4}^{(1)}\left(v_{11} ; \varepsilon\right)+(13-2 \varepsilon) I_{-3,-1}^{(1)}\left(v_{12}, v_{11} ; \varepsilon\right)\right. \\
&\left.-3 v_{11} I_{-2,-1}^{(1)}\left(v_{12}, v_{11} ; \varepsilon\right)-5 v_{12} I_{-3}^{(1)}\left(v_{11} ; \varepsilon\right)\right] \\
&= \frac{2 \pi}{15}\left(80-32 v_{12}-48 v_{11}+3 v_{11}^{2}+12 v_{12} v_{11}\right) \\
&+\frac{4 \pi}{225}\left(1570-676 v_{12}-1014 v_{11}+69 v_{11}^{2}+276 v_{12} v_{11}\right) \varepsilon \\
&+\mathcal{O}\left(\varepsilon^{2}\right) \\
& I_{-4,-2}^{(1)}\left(v_{12}, v_{11} ; \varepsilon\right)= \frac{1}{2(7-2 \varepsilon)}\left[2(5-\varepsilon) I_{-4,-1}^{(1)}\left(v_{12}, v_{11} ; \varepsilon\right)+2(7-\varepsilon) I_{-3,-2}^{(1)}\left(v_{12}, v_{11} ; \varepsilon\right)\right. \\
&\left.-3 v_{11} I_{-2,-2}^{(1)}\left(v_{12}, v_{11} ; \varepsilon\right)-6 v_{12} I_{-3,-1}^{(1)}\left(v_{12}, v_{11} ; \varepsilon\right)\right] \\
&= \frac{8 \pi}{105}\left(240-160 v_{12}-120 v_{11}+6 v_{11}^{2}+48 v_{12} v_{11}+24 v_{12}^{2}-3 v_{12}^{2} v_{11}\right) \\
&+\frac{4 \pi}{11025}\left(137880-97520 v_{12}-73140 v_{11}+3909 v_{11}^{2}+31272 v_{12} v_{11}\right. \\
&\left.+15636 v_{12}^{2}-2112 v_{12}^{2} v_{11}\right) \varepsilon+\mathcal{O}\left(\varepsilon^{2}\right), \\
& \frac{1}{4(4-\varepsilon)}\left[(13-2 \varepsilon) I_{-4,-2}^{(1)}\left(v_{12}, v_{11} ; \varepsilon\right)+(15-2 \varepsilon) I_{-3,-3}^{(1)}\left(v_{12}, v_{11} ; \varepsilon\right)\right. \\
&\left.-3 v_{11} I_{-2,-3}^{(1)}\left(v_{12}, v_{11} ; \varepsilon\right)-7 v_{12} I_{-3,-2}^{(1)}\left(v_{12}, v_{11} ; \varepsilon\right)\right] \\
&= \frac{4 \pi}{35}\left(280-240 v_{12}-120 v_{11}+5 v_{11}^{2}+60 v_{11} v_{12}+60 v_{12}^{2}\right. \\
&\left.-4 v_{12}^{3}-6 v_{11} v_{12}^{2}\right)+\frac{4 \pi}{3675}\left(83580-75240 v_{12}-37620 v_{11}+1655 v_{11}^{2}\right. \\
&\left.+19860 v_{11} v_{12}+19860 v_{12}^{2}-1408 v_{12}^{3}-2112 v_{11} v_{12}^{2}\right) \varepsilon+\mathcal{O}\left(\varepsilon^{2}\right) \\
& I_{-4,-3}^{(1)}\left(v_{12}, v_{11} ; \varepsilon\right)
\end{aligned}
$$




$$
\begin{aligned}
& I_{-4,-4}^{(1)}\left(v_{12}, v_{11} ; \varepsilon\right)=\frac{1}{2(9-2 \varepsilon)}\left[2(8-\varepsilon) I_{-4,-3}^{(1)}\left(v_{12}, v_{11} ; \varepsilon\right)+2(8-\varepsilon) I_{-3,-4}^{(1)}\left(v_{12}, v_{11} ; \varepsilon\right)\right. \\
& \left.-3 v_{11} I_{-2,-4}^{(1)}\left(v_{12}, v_{11} ; \varepsilon\right)-8 v_{12} I_{-3,-3}^{(1)}\left(v_{12}, v_{11} ; \varepsilon\right)\right] \\
& =\frac{16 \pi}{315}\left(1120-1120 v_{12}-420 v_{11}+15 v_{11}^{2}+240 v_{11} v_{12}+360 v_{12}^{2}\right. \\
& \left.-30 v_{11} v_{12}^{2}-40 v_{12}^{3}+v_{12}^{4}\right)+\frac{8 \pi}{99225}\left(2074520-2162720 v_{12}\right. \\
& -811020 v_{11}+30315 v_{11}^{2}+727560 v_{12}^{2}+485040 v_{11} v_{12}-63780 v_{11} v_{12}^{2} \\
& \left.-85040 v_{12}^{3}+2252 v_{12}^{4}\right) \varepsilon+\mathcal{O}\left(\varepsilon^{2}\right), \\
& I_{-4,1}^{(1)}\left(v_{12}, v_{11} ; \varepsilon\right)=\left(1-v_{12}\right) I_{-3}^{(1)}\left(v_{11} ; \varepsilon\right)+\frac{1}{2(2-\varepsilon)}\left[3\left(v_{12}-v_{11}\right) I_{-2}^{(1)}\left(v_{11} ; \varepsilon\right)\right. \\
& \left.-3 v_{12}^{2} I_{-2,1}^{(1)}\left(v_{11}, v_{12} ; \varepsilon\right)+(7-2 \varepsilon) I_{-3,1}^{(1)}\left(v_{12}, v_{11} ; \varepsilon\right)\right] \\
& =-\frac{\pi}{\varepsilon} v_{12}^{4}+\frac{\pi}{6}\left(24+16 v_{12}-24 v_{11}+3 v_{11}^{2}-12 v_{11} v_{12}+12 v_{12}^{2}\right. \\
& \left.-6 v_{11} v_{12}^{2}+12 v_{12}^{3}-25 v_{12}^{4}\right)+\frac{\pi}{36}\left(336+256 v_{12}-384 v_{11}+57 v_{11}^{2}\right. \\
& \left.-228 v_{11} v_{12}+228 v_{12}^{2}-150 v_{11} v_{12}^{2}+300 v_{12}^{3}-415 v_{12}^{4}\right) \varepsilon \\
& +\mathcal{O}\left(\varepsilon^{2}\right) \\
& I_{-4,2}^{(1)}\left(v_{12}, v_{11} ; \varepsilon\right)=\left(1-v_{12}\right) I_{-3,1}^{(1)}\left(v_{12}, v_{11} ; \varepsilon\right)+\frac{1}{(3-2 \varepsilon)}\left[3\left(v_{12}-v_{11}\right) I_{-2,1}^{(1)}\left(v_{11}, v_{12} ; \varepsilon\right)\right. \\
& \left.-3 v_{12}^{2} I_{-2,2}^{(1)}\left(v_{11}, v_{12} ; \varepsilon\right)+2(3-\varepsilon) I_{-3,2}^{(1)}\left(v_{12}, v_{11} ; \varepsilon\right)\right] \\
& =\frac{2 \pi}{\varepsilon} v_{12}^{2}\left(3 v_{11}-8 v_{12}+5 v_{12}^{2}\right)+\frac{\pi}{3}\left(8+16 v_{12}-12 v_{11}+3 v_{11}^{2}-24 v_{11} v_{12}\right. \\
& \left.+36 v_{12}^{2}+66 v_{11} v_{12}^{2}-176 v_{12}^{3}+80 v_{12}^{4}\right)+\frac{\pi}{9}\left(52+128 v_{12}-96 v_{11}\right. \\
& \left.+33 v_{11}^{2}-264 v_{11} v_{12}+396 v_{12}^{2}+510 v_{11} v_{12}^{2}-1360 v_{12}^{3}+610 v_{12}^{4}\right) \varepsilon \\
& +\mathcal{O}\left(\varepsilon^{2}\right) \\
& I_{-3,-1}^{(1)}\left(v_{12}, v_{11} ; \varepsilon\right)=\frac{1}{5-2 \varepsilon}\left[(3-\varepsilon) I_{-3}^{(1)}\left(v_{11} ; \varepsilon\right)+(5-\varepsilon) I_{-2,-1}^{(1)}\left(v_{11}, v_{12} ; \varepsilon\right)\right. \\
& \left.-v_{11} I_{-1,-1}^{(1)}\left(v_{11}, v_{12} ; \varepsilon\right)-2 v_{12} I_{-2}^{(1)}\left(v_{11} ; \varepsilon\right)\right] \\
& =\frac{2 \pi}{5}\left(16-6 v_{12}-6 v_{11}+v_{12} v_{11}\right) \\
& +\frac{4 \pi}{75}\left(298-123 v_{12}-123 v_{11}+23 v_{11} v_{12}\right) \varepsilon \\
& +\mathcal{O}\left(\varepsilon^{2}\right) \\
& I_{-3,-2}^{(1)}\left(v_{12}, v_{11} ; \varepsilon\right)=\frac{1}{4(3-\varepsilon)}\left[(9-2 \varepsilon) I_{-3,-1}^{(1)}\left(v_{11}, v_{12} ; \varepsilon\right)+(11-2 \varepsilon) I_{-2,-2}^{(1)}\left(v_{11}, v_{12} ; \varepsilon\right)\right. \\
& \left.-2 v_{11} I_{-1,-2}^{(1)}\left(v_{11}, v_{12} ; \varepsilon\right)-5 v_{12} I_{-2,-1}^{(1)}\left(v_{11}, v_{12} ; \varepsilon\right)\right]
\end{aligned}
$$




$$
\begin{aligned}
& =\frac{4 \pi}{15}\left(40-24 v_{12}-12 v_{11}+3 v_{12}^{2}+3 v_{11} v_{12}\right) \\
& +\frac{4 \pi}{225}\left(1570-1014 v_{12}-507 v_{11}+138 v_{12} v_{11}+138 v_{12}^{2}\right) \varepsilon \\
& +\mathcal{O}\left(\varepsilon^{2}\right) \\
& I_{-3,-3}^{(1)}\left(v_{12}, v_{11} ; \varepsilon\right)=\frac{1}{7-2 \varepsilon}\left[(6-\varepsilon)\left(I_{-3,-2}^{(1)}\left(v_{12}, v_{11} ; \varepsilon\right)+I_{-2,-3}^{(1)}\left(v_{12}, v_{11} ; \varepsilon\right)\right)\right. \\
& \left.-v_{11} I_{-1,-3}^{(1)}\left(v_{12}, v_{11} ; \varepsilon\right)-3 v_{12} I_{-2,-2}^{(1)}\left(v_{12}, v_{11} ; \varepsilon\right)\right] \\
& =\frac{4 \pi}{35}\left(160-120 v_{12}-40 v_{11}+12 v_{11} v_{12}+24 v_{12}^{2}-v_{12}^{3}\right) \\
& +\frac{4 \pi}{3675}\left(45960-36570 v_{12}-12190 v_{11}+3909 v_{11} v_{12}\right. \\
& \left.+7818 v_{12}^{2}-352 v_{12}^{3}\right) \varepsilon+\mathcal{O}\left(\varepsilon^{2}\right), \\
& I_{-3,-4}^{(1)}\left(v_{12}, v_{11} ; \varepsilon\right)=\frac{1}{4(4-\varepsilon)}\left[(15-2 \varepsilon) I_{-3,-3}^{(1)}\left(v_{12}, v_{11} ; \varepsilon\right)+(13-2 \varepsilon) I_{-2,-4}^{(1)}\left(v_{12}, v_{11} ; \varepsilon\right)\right. \\
& \left.-2 v_{11} I_{-1,-4}^{(1)}\left(v_{12}, v_{11} ; \varepsilon\right)-7 v_{12} I_{-2,-3}^{(1)}\left(v_{12}, v_{11} ; \varepsilon\right)\right] \\
& =\frac{16 \pi}{35}\left(70-60 v_{12}-15 v_{11}+5 v_{11} v_{12}+15 v_{12}^{2}-v_{12}^{3}\right) \\
& +\frac{8 \pi}{3675}\left(41790-37620 v_{12}-9405 v_{11}+3310 v_{11} v_{12}+9930 v_{12}^{2}\right. \\
& \left.-704 v_{12}^{3}\right) \varepsilon+\mathcal{O}\left(\varepsilon^{2}\right) \\
& I_{-3,1}^{(1)}\left(v_{12}, v_{11} ; \varepsilon\right)=\left(1-v_{12}\right) I_{-2}^{(1)}\left(v_{11} ; \varepsilon\right)+\frac{1}{3-2 \varepsilon}\left[2\left(v_{12}-v_{11}\right) I^{(0)}(\varepsilon)\right. \\
& \left.-2 v_{12}^{2} I_{-1,-1}^{(1)}\left(v_{11}, v_{12} ; \varepsilon\right)+(5-2 \varepsilon) v_{12} I_{-2,1}^{(1)}\left(v_{11}, v_{12} ; \varepsilon\right)\right] \\
& =-\frac{\pi}{\varepsilon} v_{12}^{3}+\frac{\pi}{3}\left(8+6 v_{12}-6 v_{11}-3 v_{11} v_{12}+6 v_{12}^{2}-11 v_{12}^{3}\right) \\
& +\frac{\pi}{9}\left(52+48 v_{12}-48 v_{11}-33 v_{11} v_{12}+66 v_{12}^{2}-85 v_{12}^{3}\right) \varepsilon \\
& +\mathcal{O}\left(\varepsilon^{2}\right) \text {, } \\
& I_{-3,2}^{(1)}\left(v_{12}, v_{11} ; \varepsilon\right)=\left(1-v_{12}\right) I_{-2,1}^{(1)}\left(v_{11}, v_{12} ; \varepsilon\right)+\frac{1}{1-\varepsilon}\left[\left(v_{12}-v_{11}\right) I_{-1,1}^{(1)}\left(v_{11}, v_{12} ; \varepsilon\right)\right. \\
& \left.-v_{12}^{2} I_{-1,2}^{(1)}\left(v_{11}, v_{12} ; \varepsilon\right)+(2-\varepsilon) v_{12} I_{-2,2}^{(1)}\left(v_{11}, v_{12} ; \varepsilon\right)\right] \\
& =\frac{3 \pi}{\varepsilon} v_{12}\left(v_{11}-3 v_{12}+2 v_{12}^{2}\right) \\
& +\pi\left(2+6 v_{12}-3 v_{11}+9 v_{11} v_{12}-27 v_{12}^{2}+12 v_{12}^{3}\right) \\
& +\pi\left(4+18 v_{12}-9 v_{11}+21 v_{11} v_{12}-63 v_{12}^{2}+30 v_{12}^{3}\right) \varepsilon+\mathcal{O}\left(\varepsilon^{2}\right),
\end{aligned}
$$




$$
\begin{aligned}
& I_{-2,-1}^{(1)}\left(v_{12}, v_{11} ; \varepsilon\right)=\frac{1}{4(2-\varepsilon)}\left[(5-2 \varepsilon) I_{-2}^{(1)}\left(v_{11} ; \varepsilon\right)+(7-2 \varepsilon) I_{-1,-1}^{(1)}\left(v_{12}, v_{11} ; \varepsilon\right)\right. \\
& \left.-\left(v_{11}+3 v_{12}\right) I^{(0)}(\varepsilon)\right] \\
& =\frac{2 \pi}{3}\left(6-2 v_{12}-v_{11}\right)+\frac{4 \pi}{9} \pi\left(21-8 v_{12}-4 v_{11}\right) \varepsilon+\mathcal{O}\left(\varepsilon^{2}\right), \\
& I_{-2,-2}^{(1)}\left(v_{12}, v_{11} ; \varepsilon\right)=\frac{1}{2(5-2 \varepsilon)}\left[2(4-\varepsilon)\left(I_{-2,-1}^{(1)}\left(v_{12}, v_{11} ; \varepsilon\right)+I_{-1,-2}^{(1)}\left(v_{12}, v_{11} ; \varepsilon\right)\right)\right. \\
& \left.-v_{11} I_{-2}^{(0)}(\varepsilon)-4 v_{12} I_{-1,-1}^{(1)}\left(v_{12}, v_{11} ; \varepsilon\right)\right] \\
& =\frac{4 \pi}{15}\left(24-12 v_{12}-3 v_{11}+v_{12}^{2}\right) \\
& +\frac{4 \pi}{225}\left(894-492 v_{12}-123 v_{11}+46 v_{12}^{2}\right) \varepsilon+\mathcal{O}\left(\varepsilon^{2}\right), \\
& I_{-2,-3}^{(1)}\left(v_{12}, v_{11} ; \varepsilon\right)=\frac{1}{4(3-\varepsilon)}\left[(11-2 \varepsilon) I_{-2,-2}^{(1)}\left(v_{12}, v_{11} ; \varepsilon\right)+(9-2 \varepsilon) I_{-1,-3}^{(1)}\left(v_{12}, v_{11} ; \varepsilon\right)\right) \\
& \left.-v_{11} I_{-3}^{(0)}(\varepsilon)-5 v_{12} I_{-1,-2}^{(1)}\left(v_{12}, v_{11} ; \varepsilon\right)\right] \\
& =\frac{4 \pi}{15}\left(40-24 v_{12}-4 v_{11}+3 v_{12}^{2}\right) \\
& +\frac{4 \pi}{225}\left(1570-1014 v_{12}-169 v_{11}+138 v_{12}^{2}\right) \varepsilon+\mathcal{O}\left(\varepsilon^{2}\right), \\
& I_{-2,-4}^{(1)}\left(v_{12}, v_{11} ; \varepsilon\right)=\frac{1}{2(7-2 \varepsilon)}\left[2(7-\varepsilon) I_{-2,-3}^{(1)}\left(v_{12}, v_{11} ; \varepsilon\right)+2(5-\varepsilon) I_{-1,-4}^{(1)}\left(v_{12}, v_{11} ; \varepsilon\right)\right) \\
& \left.-v_{11} I_{-4}^{(0)}(\varepsilon)-6 v_{12} I_{-1,-3}^{(1)}\left(v_{12}, v_{11} ; \varepsilon\right)\right] \\
& =\frac{32 \pi}{105}\left(60-40 v_{12}-5 v_{11}+6 v_{12}^{2}\right) \\
& +\frac{8 \pi}{11025}\left(68940-48760 v_{12}-6095 v_{11}+7818 v_{12}^{2}\right) \varepsilon+\mathcal{O}\left(\varepsilon^{2}\right), \\
& I_{-2,1}^{(1)}\left(v_{12}, v_{11} ; \varepsilon\right)=\left(1-v_{12}\right) I^{(0)}(\varepsilon)+\frac{1}{2(1-\varepsilon)}\left[\left(v_{12}-v_{11}\right) I^{(0)}(\varepsilon)\right. \\
& \left.-v_{12}^{2} I_{1}^{(0)}(\varepsilon)+(3-2 \varepsilon) v_{12} I_{-1,1}^{(1)}\left(v_{11}, v_{12} ; \varepsilon\right)\right] \\
& =-\frac{\pi}{\varepsilon} v_{12}^{2}+\pi\left(2-v_{11}+2 v_{12}-3 v_{12}^{2}\right) \\
& +\pi\left(4-3 v_{11}+6 v_{12}-7 v_{12}^{2}\right) \varepsilon+\mathcal{O}\left(\varepsilon^{2}\right), \\
& I_{-2,2}^{(1)}\left(v_{12}, v_{11} ; \varepsilon\right)=\left(1-v_{12}\right) I_{-1,1}^{(1)}\left(v_{11}, v_{12} ; \varepsilon\right)+\frac{1}{2(1-\varepsilon)}\left[\left(v_{12}-v_{11}\right) I_{1}^{(0)}(\varepsilon)\right. \\
& \left.-v_{12}^{2} I_{2}^{(0)}(\varepsilon)+(3-2 \varepsilon) v_{12} I_{-1,2}^{(1)}\left(v_{11}, v_{12} ; \varepsilon\right)\right] \\
& =\frac{\pi\left(v_{11}-4 v_{12}+3 v_{12}^{2}\right)}{\varepsilon}+\pi\left(2+2 v_{11}-8 v_{12}+3 v_{12}^{2}\right) \\
& +\pi\left(4+4 v_{11}-16 v_{12}+9 v_{12}^{2}\right) \varepsilon+\mathcal{O}\left(\varepsilon^{2}\right) .
\end{aligned}
$$




$$
\begin{aligned}
& I_{-1,-1}^{(1)}\left(v_{12}, v_{11} ; \varepsilon\right)=\frac{4-v_{12}-2 \varepsilon}{3-2 \varepsilon} I^{(0)}(\varepsilon) \\
& =\frac{2}{3} \pi\left(4-v_{12}\right)+\frac{4}{9} \pi\left(13-4 v_{12}\right) \varepsilon+\mathcal{O}\left(\varepsilon^{2}\right), \\
& I_{-1,-2}^{(1)}\left(v_{12}, v_{11} ; \varepsilon\right)=\frac{1}{4(2-\varepsilon)}\left[(7-2 \varepsilon) I_{-1,-1}^{(1)}\left(v_{12}, v_{11} ; \varepsilon\right)+(5-2 \varepsilon) I_{-2}^{(0)}(\varepsilon)-3 v_{12} I^{(0)}(\varepsilon)\right] \\
& =\frac{4 \pi}{3}\left(3-v_{12}\right)+\frac{4 \pi}{9}\left(21-8 v_{12}\right) \varepsilon+\mathcal{O}\left(\varepsilon^{2}\right), \\
& I_{-1,-3}^{(1)}\left(v_{12}, v_{11} ; \varepsilon\right)=\frac{1}{5-2 \varepsilon}\left[(5-\varepsilon) I_{-1,-2}^{(1)}\left(v_{12}, v_{11} ; \varepsilon\right)+(3-\varepsilon) I_{-3}^{(0)}(\varepsilon)-2 v_{12} I_{-2}^{(0)}(\varepsilon)\right] \\
& =\frac{4 \pi}{5}\left(8-3 v_{12}\right)+\frac{4 \pi}{75}\left(298-123 v_{12}\right) \varepsilon+\mathcal{O}\left(\varepsilon^{2}\right), \\
& I_{-1,-4}^{(1)}\left(v_{12}, v_{11} ; \varepsilon\right)=\frac{1}{4(3-\varepsilon)}\left[(13-2 \varepsilon) I_{-1,-3}^{(1)}\left(v_{12}, v_{11} ; \varepsilon\right)+(7-2 \varepsilon) I_{-4}^{(0)}(\varepsilon)-5 v_{12} I_{-3}^{(0)}(\varepsilon)\right] \\
& =\frac{32 \pi}{15}\left(5-2 v_{12}\right)+\frac{8 \pi}{225}\left(785-338 v_{12}\right) \varepsilon+\mathcal{O}\left(\varepsilon^{2}\right), \\
& I_{-1,1}^{(1)}\left(v_{12}, v_{11} ; \varepsilon\right)=\left(1-v_{12}\right) I^{(0)}(\varepsilon)+v_{12} I_{1}^{(0)}(\varepsilon) \\
& =-\frac{\pi}{\varepsilon} v_{12}+2 \pi\left(1-v_{12}\right)(1+2 \varepsilon)+\mathcal{O}\left(\varepsilon^{2}\right), \\
& I_{-1,2}^{(1)}\left(v_{12}, v_{11} ; \varepsilon\right)=\left(1-v_{12}\right) I_{1}^{(0)}(\varepsilon)+v_{12} I_{2}^{(0)}(\varepsilon) \\
& =-\frac{\pi\left(1-v_{12}\right)}{\varepsilon}-\pi v_{12}(1-\varepsilon)+\mathcal{O}\left(\varepsilon^{2}\right), \\
& I_{1,1}^{(1)}\left(v_{12}, v_{11} ; \varepsilon\right)=\frac{\pi}{v_{12}}\left[-\frac{1}{\varepsilon}-\log \frac{v_{11}}{v_{12}^{2}}-\frac{\varepsilon}{2} L\left(v_{11}, v_{12}\right)+\mathcal{O}\left(\varepsilon^{2}\right)\right], \\
& I_{1,2}^{(1)}\left(v_{12}, v_{11} ; \varepsilon\right)=\frac{\pi}{v_{12}^{3}}\left[\frac{v_{11}-v_{12}}{\varepsilon}+2 v_{11}-4 v_{12}+v_{12}^{2}+\left(v_{11}-v_{12}\right) \log \frac{v_{11}}{v_{12}^{2}}\right. \\
& +\frac{\varepsilon}{2}\left(2 v_{12}^{2}+4\left(v_{11}-v_{12}\right) \log \frac{v_{11}}{v_{12}^{2}}-4 v_{12} \sqrt{1-v_{11}} \log \left(\frac{1+\sqrt{1-v_{11}}}{1-\sqrt{1-v_{11}}}\right)\right. \\
& \left.\left.+\left(v_{11}-v_{12}\right) L\left(v_{11}, v_{12}\right)\right)\right]+\mathcal{O}\left(\varepsilon^{2}\right), \\
& I_{2,1}^{(1)}\left(v_{12}, v_{11} ; \varepsilon\right)=\frac{\pi}{v_{12}^{2} v_{11}}\left[-\frac{v_{11}}{\varepsilon}+2 v_{12}-2 v_{11}-v_{11} \log \left(\frac{v_{11}}{v_{12}^{2}}\right)\right. \\
& -\varepsilon\left(2 v_{11} \log \frac{v_{11}}{v_{12}^{2}}-2 v_{12} \sqrt{1-v_{11}} \log \left(\frac{1+\sqrt{1-v_{11}}}{1-\sqrt{1-v_{11}}}\right)\right. \\
& \left.\left.+\frac{v_{11}}{2} L\left(v_{11}, v_{12}\right)\right)\right]+\mathcal{O}\left(\varepsilon^{2}\right) \text {, } \\
& I_{2,2}^{(1)}\left(v_{12}, v_{11} ; \varepsilon\right)=\frac{\pi}{v_{12}^{4} v_{11}}\left[\frac{1}{\varepsilon}\left(3 v_{11}^{2}-4 v_{11} v_{12}+v_{11} v_{12}^{2}\right)+8 v_{11}^{2}+5 v_{11} v_{12}^{2}-16 v_{11} v_{12}+2 v_{12}^{2}\right. \\
& +\left(3 v_{11}^{2}-4 v_{11} v_{12}+v_{11} v_{12}^{2}\right) \log \frac{v_{11}}{v_{12}^{2}}+\varepsilon\left(4 v_{11}^{2}-8 v_{11} v_{12}+5 v_{11} v_{12}^{2}\right. \\
& +\left(8 v_{11}^{2}-10 v_{11} v_{12}+2 v_{11} v_{12}^{2}\right) \log \frac{v_{11}}{v_{12}^{2}} \\
& -\left(6 v_{11} v_{12}-2 v_{12}^{2}\right) \sqrt{1-v_{11}} \log \left(\frac{1+\sqrt{1-v_{11}}}{1-\sqrt{1-v_{11}}}\right) \\
& \left.\left.+\frac{1}{2}\left(3 v_{11}^{2}-4 v_{11} v_{12}+v_{11} v_{12}^{2}\right) L\left(v_{11}, v_{12}\right)\right)\right]+\mathcal{O}\left(\varepsilon^{2}\right),
\end{aligned}
$$




$$
\begin{aligned}
& I_{1,3}^{(1)}\left(v_{12}, v_{11} ; \varepsilon\right)=\frac{\pi}{2 v_{12}^{5}}\left[\frac{1}{\varepsilon}\left(-3 v_{11}^{2}+6 v_{11} v_{12}-v_{11} v_{12}^{2}-2 v_{12}^{2}\right)-8 v_{11}^{2}+22 v_{11} v_{12}-5 v_{11} v_{12}^{2}\right. \\
& -12 v_{12}^{2}+2 v_{12}^{3}+\frac{v_{12}^{4}}{2}-\left(3 v_{11}^{2}-6 v_{11} v_{12}+v_{11} v_{12}^{2}+2 v_{12}^{2}\right) \log \frac{v_{11}}{v_{12}^{2}} \\
& +\varepsilon\left(-4 v_{11}^{2}+12 v_{11} v_{12}-5 v_{11} v_{12}^{2}-8 v_{12}^{2}+4 v_{12}^{3}+\frac{3 v_{12}^{4}}{4}\right. \\
& -\left(8 v_{11}^{2}+6 v_{12}^{2}-16 v_{11} v_{12}+2 v_{11} v_{12}^{2}\right) \log \frac{v_{11}}{v_{12}^{2}} \\
& +6 v_{12}\left(v_{11}-v_{12}\right) \sqrt{1-v_{11}} \log \left(\frac{1+\sqrt{1-v_{11}}}{1-\sqrt{1-v_{11}}}\right) \\
& \left.\left.-\frac{1}{2}\left(3 v_{11}^{2}-6 v_{11} v_{12}+v_{11} v_{12}^{2}+2 v_{12}^{2}\right) L\left(v_{11}, v_{12}\right)\right)\right]+\mathcal{O}\left(\varepsilon^{2}\right), \\
& I_{3,1}^{(1)}\left(v_{12}, v_{11} ; \varepsilon\right)=\frac{\pi}{v_{12}^{3} v_{11}^{2}}\left[-\frac{v_{11}^{2}}{\varepsilon}-3 v_{11}^{2}+2 v_{11} v_{12}+2 v_{12}^{2}-v_{11} v_{12}^{2}-v_{11}^{2} \log \frac{v_{11}}{v_{12}^{2}}\right. \\
& +\frac{\varepsilon}{2}\left(4 v_{11} v_{12}+4 v_{12}^{2}-4 v_{11}^{2}-4 v_{11} v_{12}^{2}-6 v_{11}^{2} \log \frac{v_{11}}{v_{12}^{2}}+4 v_{12}\left(v_{11}+v_{12}\right)\right. \\
& \left.\left.\times \sqrt{1-v_{11}} \log \left(\frac{1+\sqrt{1-v_{11}}}{1-\sqrt{1-v_{11}}}\right)-v_{11}^{2} L\left(v_{11}, v_{12}\right)\right)\right]+\mathcal{O}\left(\varepsilon^{2}\right), \\
& I_{2,3}^{(1)}\left(v_{12}, v_{11} ; \varepsilon\right)=\frac{\pi}{8 v_{12}^{6} v_{11}}\left[\frac{12}{\varepsilon} v_{11}\left(-5 v_{11}^{2}+12 v_{11} v_{12}-6 v_{12}^{2}-3 v_{11} v_{12}^{2}+2 v_{12}^{3}\right)\right. \\
& -184 v_{11}^{3}+552 v_{11}^{2} v_{12}-384 v_{11} v_{12}^{2}-156 v_{11}^{2} v_{12}^{2}+16 v_{12}^{3}+160 v_{11} v_{12}^{3} \\
& -6 v_{11} v_{12}^{4}-12 v_{11}\left(5 v_{11}^{2}-12 v_{11} v_{12}+6 v_{12}^{2}+3 v_{11} v_{12}^{2}-2 v_{12}^{3}\right) \log \frac{v_{11}}{v_{12}^{2}} \\
& +\varepsilon\left(-144 v_{11}^{3}+448 v_{11}^{2} v_{12}-320 v_{11} v_{12}^{2}-172 v_{11}^{2} v_{12}^{2}+200 v_{11} v_{12}^{3}-13 v_{11} v_{12}^{4}\right. \\
& +8 v_{11}\left(-23 v_{11}^{2}+54 v_{11} v_{12}-27 v_{12}^{2}-12 v_{11} v_{12}^{2}+8 v_{12}^{3}\right) \log \frac{v_{11}}{v_{12}^{2}} \\
& +8 v_{12}\left(15 v_{11}^{2}-21 v_{11} v_{12}+2 v_{12}^{2}+4 v_{11} v_{12}^{2}\right) \sqrt{1-v_{11}} \log \left(\frac{1+\sqrt{1-v_{11}}}{1-\sqrt{1-v_{11}}}\right) \\
& \left.\left.-6 v_{11}\left(5 v_{11}^{2}-12 v_{11} v_{12}+6 v_{12}^{2}+3 v_{11} v_{12}^{2}-2 v_{12}^{3}\right) L\left(v_{11}, v_{12}\right)\right)\right] \\
& +\mathcal{O}\left(\varepsilon^{2}\right), \\
& I_{3,2}^{(1)}\left(v_{12}, v_{11} ; \varepsilon\right)=\frac{\pi}{v_{12}^{5} v_{11}^{2}}\left[\frac{3}{\varepsilon} v_{11}^{2}\left(2 v_{11}-3 v_{12}+v_{12}^{2}\right)+19 v_{11}^{3}+6 v_{11} v_{12}^{2}-39 v_{11}^{2} v_{12}\right. \\
& +2 v_{12}^{3}-3 v_{11} v_{12}^{3}+14 v_{11}^{2} v_{12}^{2}+3 v_{11}^{2}\left(2 v_{11}-3 v_{12}+v_{12}^{2}\right) \log \frac{v_{11}}{v_{12}^{2}} \\
& +\frac{\varepsilon}{2}\left(32 v_{11}^{3}+4 v_{12}^{3}+8 v_{11} v_{12}^{2}-68 v_{11}^{2} v_{12}-8 v_{11} v_{12}^{3}+34 v_{11}^{2} v_{12}^{2}\right. \\
& +2 v_{11}^{2}\left(19 v_{11}-27 v_{12}+8 v_{12}^{2}\right) \log \frac{v_{11}}{v_{12}^{2}} \\
& -4 v_{12}\left(6 v_{11}^{2}-3 v_{11} v_{12}-v_{12}^{2}+v_{11} v_{12}^{2}\right) \sqrt{1-v_{11}} \log \left(\frac{1+\sqrt{1-v_{11}}}{1-\sqrt{1-v_{11}}}\right) \\
& \left.\left.+3 v_{11}^{2}\left(2 v_{11}-3 v_{12}+v_{12}^{2}\right) L\left(v_{11}, v_{12}\right)\right)\right]+\mathcal{O}\left(\varepsilon^{2}\right),
\end{aligned}
$$




$$
\begin{aligned}
I_{3,3}^{(1)}\left(v_{12}, v_{11} ; \varepsilon\right) & =\frac{\pi}{4 v_{12}^{7} v_{11}^{2}}\left[-\frac{6}{\varepsilon} v_{11}^{2}\left(15 v_{11}^{2}-40 v_{11} v_{12}+24 v_{12}^{2}+12 v_{11} v_{12}^{2}-12 v_{12}^{3}+v_{12}^{4}\right)\right. \\
- & 306 v_{11}^{4}+976 v_{11}^{3} v_{12}-768 v_{11}^{2} v_{12}^{2}-312 v_{11}^{3} v_{12}^{2}+48 v_{11} v_{12}^{3}+420 v_{11}^{2} v_{12}^{3} \\
+ & 8 v_{12}^{4}-24 v_{11} v_{12}^{4}-43 v_{11}^{2} v_{12}^{4}-6 v_{11}^{2}\left(15 v_{11}^{2}-40 v_{11} v_{12}+12 v_{11} v_{12}^{2}\right. \\
+ & \left.24 v_{12}^{2}-12 v_{12}^{3}+v_{12}^{4}\right) \log \frac{v_{11}}{v_{12}^{2}}+\frac{\varepsilon}{2}\left(-616 v_{11}^{4}+2024 v_{11}^{3} v_{12}-1616 v_{11}^{2} v_{12}^{2}\right. \\
& -784 v_{11}^{3} v_{12}^{2}+48 v_{11} v_{12}^{3}+1120 v_{11}^{2} v_{12}^{3}+16 v_{12}^{4}-48 v_{11} v_{12}^{4}-145 v_{11}^{2} v_{12}^{4} \\
+ & 2 v_{11}^{2}\left(-306 v_{11}^{2}+796 v_{11} v_{12}-468 v_{12}^{2}-222 v_{11} v_{12}^{2}+216 v_{12}^{3}-16 v_{12}^{4}\right) \log \frac{v_{11}}{v_{12}^{2}} \\
+ & 8 v_{12}\left(21 v_{11}^{2} v_{12}^{2}+12 v_{11} v_{12}^{2}+2 v_{12}^{3}+45 v_{11}^{3}-75 v_{11}^{2} v_{12}-5 v_{11} v_{12}^{3}\right) \\
& \times \sqrt{1-v_{11}} \log \left(\frac{1+\sqrt{1-v_{11}}}{1-\sqrt{1-v_{11}}}\right)-6 v_{11}^{2}\left(15 v_{11}^{2}-40 v_{11} v_{12}+24 v_{12}^{2}\right. \\
+ & \left.\left.\left.12 v_{11} v_{12}^{2}-12 v_{12}^{3}+v_{12}^{4}\right) L\left(v_{11}, v_{12}\right)\right)\right]+\mathcal{O}\left(\varepsilon^{2}\right),
\end{aligned}
$$

where

$$
L\left(v_{11}, v_{12}\right)=\log ^{2} \frac{v_{11}}{v_{12}^{2}}+4 \operatorname{Li}_{2}\left(1-\frac{v_{12}}{1-\sqrt{1-v_{11}}}\right)+4 \operatorname{Li}_{2}\left(1-\frac{v_{12}}{1+\sqrt{1-v_{11}}}\right) .
$$

\section{G.5 Double massive integral with two denominators}

In this section we briefly review how we proceed with double massive integrals with two denominators. As we showed in section 3.4.4, the $\varepsilon$ expansion of the double massive master integral with two denominators $I_{1,1}^{(2)}\left(v_{12}, v_{11}, v_{22} ; \varepsilon\right)$ is generated by analytically using eq. (3.192). Eq. (3.194) explicitly illustrates the $\varepsilon$ expansion of this integral up to second order. All other double massive integrals are obtained using recursive relations (3.150), (3.152), (3.154), and (3.157) and $\varepsilon$-expansion of the master integrals $I^{(0)}$, $I_{1,0}^{(1)}\left(v_{11} ; \varepsilon\right)$, and $I_{1,1}^{(2)}\left(v_{12}, v_{11}, v_{22} ; \varepsilon\right)$.

\section{G.6 Mirkes type III integrals}

Mirkes type III integrals are defined as

$$
I_{D}^{j, l}(A, B, C)=\int \mathrm{d} \Omega_{k_{1} k_{2}} \frac{\left(-\cos \theta_{1}\right)^{l}}{\left(A+B \cos \theta_{1}+C \sin \theta_{1} \cos \theta_{2}\right)^{j}}=A^{-j} I_{D}^{j, l}\left(b, \rho^{2}\right),
$$

where $b=B / A, c=C / A$, and $\rho^{2}=b^{2}+c^{2}$.

By application of the recursion relations (3.223)-(3.226) we find the integrals with specific values of indices $j=1,2$ and $l=0, \ldots, 4$ :

$$
\begin{aligned}
I_{D}^{1,0}\left(b, \rho^{2}\right)= & I_{1}^{(1)}, \\
I_{D}^{1,1}\left(b, \rho^{2}\right)= & \frac{b}{\rho^{2}}\left[-I^{(0)}+I_{1}^{(1)}\right], \\
I_{D}^{1,2}\left(b, \rho^{2}\right)= & \frac{1}{2 \rho^{4}(1-\varepsilon)}\left[I^{(0)}\left(\rho^{2}-b^{2}(3-2 \varepsilon)\right)\right. \\
& \left.-I_{1}^{(1)}\left(\rho^{2}\left(1-\rho^{2}\right)-b^{2}\left(3-\rho^{2}-2 \varepsilon\right)\right)\right],
\end{aligned}
$$




$$
\begin{aligned}
& I_{D}^{1,3}\left(b, \rho^{2}\right)=\frac{b}{2 \rho^{6}(1-\varepsilon)}\left[\frac { I ^ { ( 0 ) } } { 3 - 2 \varepsilon } \left(\rho^{2}(1-\varepsilon)\left(9-6 \rho^{2}+4 b^{2}-2 \varepsilon\right)\right.\right. \\
& \left.\left.-b^{2}(3-2 \varepsilon)(5-2 \varepsilon)\right)-I_{1}^{(1)}\left(3 \rho^{2}\left(1-\rho^{2}\right)-b^{2}\left(5-3 \rho^{2}-2 \varepsilon\right)\right)\right], \\
& I_{D}^{1,4}\left(b, \rho^{2}\right)=\frac{1}{4 \rho^{8}(1-\varepsilon)(2-\varepsilon)}\left[\frac { I ^ { ( 0 ) } } { 3 - 2 \varepsilon } \left(-b^{4}(3-2 \varepsilon)(5-2 \varepsilon)(7-2 \varepsilon)\right.\right. \\
& -3 \rho^{4}(3-2 \varepsilon)+3 \rho^{6}(5-4 \varepsilon)+b^{4} \rho^{2}(5-2 \varepsilon)(11-10 \varepsilon) \\
& \left.-6 b^{2} \rho^{4}\left(13-16 \varepsilon+4 \varepsilon^{2}\right)+6 b^{2} \rho^{2}(3-2 \varepsilon)(5-2 \varepsilon)\right) \\
& +I_{1}^{(1)}\left(3 \rho^{4}\left(1-\rho^{2}+b^{2}\right)^{2}-6 b^{2} \rho^{2}\left(1-\rho^{2}+b^{2}\right)(5-2 \varepsilon)\right. \\
& \left.\left.+b^{4}(5-2 \varepsilon)(7-2 \varepsilon)\right)\right] \text {, } \\
& I_{D}^{2,0}\left(b, \rho^{2}\right)=\frac{1}{1-\rho^{2}}\left[(1-2 \varepsilon) I^{(0)}+2 \varepsilon I_{1}^{(1)}\right], \\
& I_{D}^{2,1}\left(b, \rho^{2}\right)=\frac{b}{\rho^{2}\left(1-\rho^{2}\right)}\left[(1-2 \varepsilon) I^{(0)}-\left(1-\rho^{2}-2 \varepsilon\right) I_{1}^{(1)}\right], \\
& I_{D}^{2,2}\left(b, \rho^{2}\right)=I^{(0)}\left[-\frac{1}{\rho^{2}}+\frac{b^{2}\left(3-2 \rho^{2}-2 \varepsilon\right)}{\rho^{4}\left(1-\rho^{2}\right)}\right] \\
& +I_{1}^{(1)}\left[\frac{\rho^{2}-3 b^{2}}{\rho^{4}}+\frac{2 b^{2} \varepsilon}{\rho^{4}\left(1-\rho^{2}\right)}\right] \text {, } \\
& I_{D}^{2,3}\left(b, \rho^{2}\right)=\frac{b}{2 \rho^{6}\left(1-\rho^{2}\right)(1-\varepsilon)}\left[I ^ { ( 0 ) } \left(-3 \rho^{2}\left(1-\rho^{2}\right)(3-2 \varepsilon)\right.\right. \\
& \left.+b^{2}(3-2 \varepsilon)(5-2 \varepsilon)-b^{2} \rho^{2}(13-10 \varepsilon)\right) \\
& +I_{1}^{(1)}\left(3 \rho^{2}\left(1-\rho^{2}\right)\left(3-\rho^{2}-2 \varepsilon\right)\right. \\
& \left.\left.-b^{2}\left((3-2 \varepsilon)(5-2 \varepsilon)-3 \rho^{2}\left(6-\rho^{2}-4 \varepsilon\right)\right)\right)\right] \text {, } \\
& I_{D}^{2,4}\left(b, \rho^{2}\right)=\frac{I^{(0)}}{2 \rho^{2}}\left[\frac{3}{\rho^{2}(1-\varepsilon)}-\frac{6}{3-2 \varepsilon}+\frac{6 b^{2}}{\rho^{2}}\left(\frac{4}{3-2 \varepsilon}-\frac{5-2 \varepsilon}{\rho^{2}(1-\varepsilon)}\right)\right. \\
& \left.+\frac{b^{4}}{\rho^{2}\left(1-\rho^{2}\right)}\left(\frac{16}{3-2 \varepsilon}+\frac{(5-2 \varepsilon)(7-2 \varepsilon)}{\rho^{4}(1-\varepsilon)}-\frac{(5-2 \varepsilon)(23-18 \varepsilon)}{\rho^{2}(1-\varepsilon)(3-2 \varepsilon)}\right)\right] \\
& +\frac{I_{1}^{(1)}}{2 \rho^{8}}\left[4 b^{2}\left(3 \beta^{2}-5 b^{2}\right)-\frac{3\left(1-\rho^{2}\right)\left(b^{2}-\rho^{2}\right)\left(5 b^{2}-\rho^{2}\right)}{1-\varepsilon}+\frac{4 b^{4} \varepsilon}{1-\rho^{2}}\right] \text {. }
\end{aligned}
$$


Open Access. This article is distributed under the terms of the Creative Commons Attribution License (CC-BY 4.0), which permits any use, distribution and reproduction in any medium, provided the original author(s) and source are credited.

\section{References}

[1] G. 't Hooft and M.J.G. Veltman, Regularization and Renormalization of Gauge Fields, Nucl. Phys. B 44 (1972) 189 [InSPIRE].

[2] G. 't Hooft and M.J.G. Veltman, Scalar One Loop Integrals, Nucl. Phys. B 153 (1979) 365 [INSPIRE].

[3] G. Passarino and M.J.G. Veltman, One Loop Corrections for $e^{+} e^{-}$Annihilation Into $\mu^{+} \mu^{-}$ in the Weinberg Model, Nucl. Phys. B 160 (1979) 151 [INSPIRE].

[4] R.K. Ellis, D.A. Ross and A.E. Terrano, The Perturbative Calculation of Jet Structure in $e^{+} e^{-}$Annihilation, Nucl. Phys. B 178 (1981) 421 [inSPIRE].

[5] W.L. van Neerven and J.A.M. Vermaseren, Large loop integrals, Phys. Lett. B 137 (1984) 241 [INSPIRE].

[6] R.G. Stuart, Algebraic Reduction of One Loop Feynman Diagrams to Scalar Integrals, Comput. Phys. Commun. 48 (1988) 367 [InSPIRE].

[7] G.J. van Oldenborgh and J.A.M. Vermaseren, New Algorithms for One Loop Integrals, Z. Phys. C 46 (1990) 425 [INSPIRE].

[8] J.G. Korner and P. Sieben, Use of helicity methods in evaluating loop integrals: $A$ QCD example, Nucl. Phys. B 363 (1991) 65 [INSPIRE].

[9] J.G. Korner, J.H. Kühn, M. Krammer and H. Schneider, Zweig Forbidden Radiative Orthoquarkonium Decays in Perturbative QCD, Nucl. Phys. B 229 (1983) 115 [InSPIRE].

[10] E.E. Boos and A.I. Davydychev, A Method of evaluating massive Feynman integrals, Theor. Math. Phys. 89 (1991) 1052 [INSPIRE].

[11] A.I. Davydychev, A Simple formula for reducing Feynman diagrams to scalar integrals, Phys. Lett. B 263 (1991) 107 [INSPIRE].

[12] Z. Bern, L.J. Dixon and D.A. Kosower, Dimensionally regulated pentagon integrals, Nucl. Phys. B 412 (1994) 751 [hep-ph/9306240] [INSPIRE].

[13] Z. Bern, L.J. Dixon, D.C. Dunbar and D.A. Kosower, One loop $n$ point gauge theory amplitudes, unitarity and collinear limits, Nucl. Phys. B 425 (1994) 217 [hep-ph/9403226] [INSPIRE].

[14] Z. Bern, L.J. Dixon, D.C. Dunbar and D.A. Kosower, Fusing gauge theory tree amplitudes into loop amplitudes, Nucl. Phys. B 435 (1995) 59 [hep-ph/9409265] [InSPIRE].

[15] J.M. Campbell, E.W.N. Glover and D.J. Miller, One loop tensor integrals in dimensional regularization, Nucl. Phys. B 498 (1997) 397 [hep-ph/9612413] [INSPIRE].

[16] R. Pittau, A Simple method for multileg loop calculations, Comput. Phys. Commun. 104 (1997) 23 [hep-ph/9607309] [INSPIRE].

[17] G. Devaraj and R.G. Stuart, Reduction of one loop tensor form-factors to scalar integrals: $A$ General scheme, Nucl. Phys. B 519 (1998) 483 [hep-ph/9704308] [INSPIRE]. 
[18] O.V. Tarasov, Connection between Feynman integrals having different values of the space-time dimension, Phys. Rev. D 54 (1996) 6479 [hep-th/9606018] [INSPIRE].

[19] J. Fleischer, F. Jegerlehner and O.V. Tarasov, Algebraic reduction of one loop Feynman graph amplitudes, Nucl. Phys. B 566 (2000) 423 [hep-ph/9907327] [INSPIRE].

[20] G. Duplancic and B. Nizic, Dimensionally regulated one loop box scalar integrals with massless internal lines, Eur. Phys. J. C 20 (2001) 357 [hep-ph/0006249] [InSPIRE].

[21] S. Laporta, High precision calculation of multiloop Feynman integrals by difference equations, Int. J. Mod. Phys. A 15 (2000) 5087 [hep-ph/0102033] [InSPIRE].

[22] F. Cachazo, P. Svrček and E. Witten, Twistor space structure of one-loop amplitudes in gauge theory, JHEP 10 (2004) 074 [hep-th/0406177] [INSPIRE].

[23] R. Britto, F. Cachazo and B. Feng, Generalized unitarity and one-loop amplitudes in $N=4$ super-Yang-Mills, Nucl. Phys. B 725 (2005) 275 [hep-th/0412103] [INSPIRE].

[24] V.A. Smirnov, Feynman integral calculus, Springer, Berlin, Germany (2006) [DOI].

[25] V.A. Smirnov, Analytic tools for Feynman integrals, Springer Tracts Mod. Phys. 250 (2012) 1 [INSPIRE].

[26] A. Denner and S. Dittmaier, Reduction schemes for one-loop tensor integrals, Nucl. Phys. B 734 (2006) 62 [hep-ph/0509141] [INSPIRE].

[27] G. Ossola, C.G. Papadopoulos and R. Pittau, Reducing full one-loop amplitudes to scalar integrals at the integrand level, Nucl. Phys. B 763 (2007) 147 [hep-ph/0609007] [INSPIRE].

[28] R.K. Ellis, W.T. Giele and Z. Kunszt, A Numerical Unitarity Formalism for Evaluating One-Loop Amplitudes, JHEP 03 (2008) 003 [arXiv:0708.2398] [INSPIRE].

[29] A. Faessler et al., Magnetic moments of heavy baryons in the relativistic three-quark model, Phys. Rev. D 73 (2006) 094013 [hep-ph/0602193] [INSPIRE].

[30] T. Gutsche, M.A. Ivanov, J.G. Körner, V.E. Lyubovitskij and Z. Tyulemissov, Ab initio three-loop calculation of the $W$-exchange contribution to nonleptonic decays of double charm baryons, Phys. Rev. D 99 (2019) 056013 [arXiv:1812.09212] [InSPIRE].

[31] D. Forde, Direct extraction of one-loop integral coefficients, Phys. Rev. D 75 (2007) 125019 [arXiv:0704.1835] [INSPIRE].

[32] W.T. Giele, Z. Kunszt and K. Melnikov, Full one-loop amplitudes from tree amplitudes, JHEP 04 (2008) 049 [arXiv:0801.2237] [INSPIRE].

[33] A.S. Zhevlakov, M. Gorchtein, A.N. Hiller Blin, T. Gutsche and V.E. Lyubovitskij, Bounds on rare decays of $\eta$ and $\eta^{\prime}$ mesons from the neutron EDM, Phys. Rev. D 99 (2019) 031703 [arXiv: 1812.00171] [INSPIRE].

[34] M.Y. Kalmykov, Gauss hypergeometric function: Reduction, $\epsilon$-expansion for integer/half-integer parameters and Feynman diagrams, JHEP 04 (2006) 056 [hep-th/0602028] [INSPIRE].

[35] R.K. Ellis and G. Zanderighi, Scalar one-loop integrals for QCD, JHEP 02 (2008) 002 [arXiv:0712.1851] [INSPIRE].

[36] A. Denner and S. Dittmaier, Scalar one-loop 4-point integrals, Nucl. Phys. B 844 (2011) 199 [arXiv: 1005.2076] [INSPIRE]. 
[37] R.K. Ellis, Z. Kunszt, K. Melnikov and G. Zanderighi, One-loop calculations in quantum field theory: from Feynman diagrams to unitarity cuts, Phys. Rept. 518 (2012) 141 [arXiv: 1105.4319] [INSPIRE].

[38] P. Mastrolia, T. Peraro and A. Primo, Adaptive Integrand Decomposition in parallel and orthogonal space, JHEP 08 (2016) 164 [arXiv:1605.03157] [INSPIRE].

[39] T. Peraro and L. Tancredi, Tensor decomposition for bosonic and fermionic scattering amplitudes, Phys. Rev. D 103 (2021) 054042 [arXiv: 2012.00820] [InSPIRE].

[40] J. Davies, G. Mishima, M. Steinhauser and D. Wellmann, $g g \rightarrow Z Z$ : analytic two-loop results for the low- and high-energy regions, JHEP 04 (2020) 024 [arXiv:2002.05558] [INSPIRE].

[41] M. Kalmykov, V. Bytev, B.A. Kniehl, S.-O. Moch, B.F.L. Ward and S.A. Yost, Hypergeometric Functions and Feynman Diagrams, in Antidifferentiation and the Calculation of Feynman Amplitudes, (2020) [arXiv:2012.14492] [INSPIRE].

[42] D. Boer and W. Vogelsang, Drell-Yan lepton angular distribution at small transverse momentum, Phys. Rev. D 74 (2006) 014004 [hep-ph/0604177] [INSPIRE].

[43] E.L. Berger, J.-W. Qiu and R.A. Rodriguez-Pedraza, Transverse momentum dependence of the angular distribution of the Drell-Yan process, Phys. Rev. D 76 (2007) 074006 [arXiv:0708.0578] [INSPIRE].

[44] W.L. van Neerven, Dimensional Regularization of Mass and Infrared Singularities in Two Loop On-shell Vertex Functions, Nucl. Phys. B 268 (1986) 453 [INSPIRE].

[45] A. Devoto, D.W. Duke, J.D. Kimel and G.A. Sowell, Analytic Calculation of the Fourth Order Quantum Chromodynamic Contribution to the Nonsinglet Quark Longitudinal Structure Function, Phys. Rev. D 30 (1984) 541 [INSPIRE].

[46] J. Smith, D. Thomas and W.L. van Neerven, QCD Corrections to the Reaction $p \bar{p} \rightarrow W \gamma X$, Z. Phys. C 44 (1989) 267 [INSPIRE].

[47] J. Smith, On Angular Integrals in n-Dimensions, Preprint YITP-SB-09-13 (2009).

[48] G. Somogyi, Angular integrals in d dimensions, J. Math. Phys. 52 (2011) 083501 [arXiv: 1101.3557] [INSPIRE].

[49] E. Mirkes, Angular decay distribution of leptons from $W$ bosons at NLO in hadronic collisions, Nucl. Phys. B 387 (1992) 3 [InSPIRE].

[50] C. Anastasiou and K. Melnikov, Higgs boson production at hadron colliders in NNLO QCD, Nucl. Phys. B 646 (2002) 220 [hep-ph/0207004] [INSPIRE].

[51] A. Mitov, A New method for calculating differential distributions directly in Mellin space, Phys. Lett. B 643 (2006) 366 [hep-ph/0511340] [INSPIRE].

[52] D. Bonocore, E. Laenen and R. Rietkerk, Unitarity methods for Mellin moments of Drell-Yan cross sections, JHEP 05 (2016) 079 [arXiv: 1603.05252] [INSPIRE].

[53] M. Argeri and P. Mastrolia, Feynman Diagrams and Differential Equations, Int. J. Mod. Phys. A 22 (2007) 4375 [arXiv:0707.4037] [INSPIRE].

[54] S. Moch and C. Schneider, Feynman integrals and difference equations, PoS ACAT (2007) 083 [arXiv:0709.1769] [INSPIRE].

[55] A.N. Schellekens, Perturbative QCD and lepton Pair Production, Ph.D. Thesis, RX-953, Nijmegen (1981). 
[56] T. Matsuura, S.C. van der Marck and W.L. van Neerven, The Calculation of the Second Order Soft and Virtual Contributions to the Drell-Yan Cross-Section, Nucl. Phys. B 319 (1989) 570 [INSPIRE].

[57] T. Matsuura, R. Hamberg and W.L. van Neerven, The Contribution of the Gluon-gluon Subprocess to the Drell-Yan K Factor, Nucl. Phys. B 345 (1990) 331 [InSPIRE].

[58] R. Hamberg, W.L. van Neerven and T. Matsuura, A complete calculation of the order $\alpha_{s}^{2}$ correction to the Drell-Yan K factor, Nucl. Phys. B 359 (1991) 343 [Erratum ibid. 644 (2002) 403] [INSPIRE].

[59] N. Bahjat-Abbas, J. Sinninghe Damsté, L. Vernazza and C.D. White, On next-to-leading power threshold corrections in Drell-Yan production at $N^{3} L O$, JHEP 10 (2018) 144 [arXiv: 1807.09246] [INSPIRE].

[60] D.W. Duke and J.F. Owens, Quantum Chromodynamics Corrections to Deep Inelastic Compton Scattering, Phys. Rev. D 26 (1982) 1600 [Erratum ibid. 28 (1983) 1227] [INSPIRE].

[61] D. Anderle, D. de Florian and Y. Rotstein Habarnau, Towards semi-inclusive deep inelastic scattering at next-to-next-to-leading order, Phys. Rev. D 95 (2017) 034027 [arXiv: 1612.01293] [INSPIRE].

[62] B. Wang, J.O. Gonzalez-Hernandez, T.C. Rogers and N. Sato, Large Transverse Momentum in Semi-Inclusive Deeply Inelastic Scattering Beyond Lowest Order, Phys. Rev. D 99 (2019) 094029 [arXiv: 1903.01529] [INSPIRE].

[63] L.E. Gordon and W. Vogelsang, Polarized and unpolarized prompt photon production beyond the leading order, Phys. Rev. D 48 (1993) 3136 [INSPIRE].

[64] R.K. Ellis, M.A. Furman, H.E. Haber and I. Hinchliffe, Large Corrections to High $p_{T}$ Hadron-Hadron Scattering in QCD, Nucl. Phys. B 173 (1980) 397 [InSPIRE].

[65] W. Beenakker, H. Kuijf, W.L. van Neerven and J. Smith, QCD Corrections to Heavy Quark Production in pp Collisions, Phys. Rev. D 40 (1989) 54 [InSPIRE].

[66] I. Bojak, NLO QCD corrections to the polarized photoproduction and hadroproduction of heavy quarks, hep-ph/0005120 [INSPIRE].

[67] G. Isidori, S. Nabeebaccus and R. Zwicky, QED corrections in $\bar{B} \rightarrow \bar{K} \ell^{+} \ell^{-}$at the double-differential level, JHEP 12 (2020) 104 [arXiv: 2009.00929] [INSPIRE].

[68] P. Bolzoni, G. Somogyi and Z. Trócsányi, A subtraction scheme for computing QCD jet cross sections at NNLO: integrating the iterated singly-unresolved subtraction terms, JHEP 01 (2011) 059 [arXiv: 1011.1909] [INSPIRE].

[69] S. Lionetti, Subtraction of Infrared Singularities at Higher Orders in QCD, Ph.D. Thesis, ETH Zürich (2018).

[70] C. Specchia, Perturbative Corrections to Inclusive and Differential Cross Sections for Higgs Production at the LHC, Ph.D. Thesis, ETH Zürich (2018).

[71] M. Höschele, Phasenraum-Masterintegrale zur Berechnung der Higgsproduktion in Gluonfusion, Ph.D. Thesis, KIT Karlsruhe (2018).

[72] C. Anastasiou, C. Duhr, F. Dulat and B. Mistlberger, Soft triple-real radiation for Higgs production at N3LO, JHEP 07 (2013) 003 [arXiv:1302.4379] [INSPIRE].

[73] D. Baranowski, NNLO zero-jettiness beam and soft functions to higher orders in the dimensional-regularization parameter $\epsilon$, Eur. Phys. J. C 80 (2020) 523 [arXiv:2004.03285] [INSPIRE]. 
[74] F.M. Ringer, Threshold Resummation and Higher Order Effects in QCD, Ph.D. Thesis, Tübingen University (2015).

[75] F. Ringer and W. Vogelsang, Single-Spin Asymmetries in $W$ Boson Production at Next-to-Leading Order, Phys. Rev. D 91 (2015) 094033 [arXiv: 1503.07052] [InSPIRE].

[76] M. Schlegel, Partonic description of the transverse target single-spin asymmetry in inclusive deep-inelastic scattering, Phys. Rev. D 87 (2013) 034006 [arXiv:1211.3579] [InSPIRE].

[77] W. Kotlarski, Sgluons in the same-sign lepton searches, JHEP 02 (2017) 027 [arXiv: 1608.00915] [INSPIRE].

[78] B. Lillard, T.M.P. Tait and P. Tanedo, Kaluza-Klein gluons at $100 \mathrm{TeV}$ : NLO corrections, Phys. Rev. D 94 (2016) 054012 [arXiv:1602.08622] [INSPIRE].

[79] P. Hinderer, Higher Order Studies in Perturbative QCD, Ph.D. Thesis, Tübingen University (2017).

[80] F. Hekhorn, Next-to-Leading Order QCD Corrections to Heavy-Flavour Production in Neutral Current DIS, Ph.D. Thesis, Tübingen University (2019).

[81] J. Blümlein, A. De Freitas, C. Raab and K. Schönwald, The $O\left(\alpha^{2}\right)$ initial state QED corrections to $e^{+} e^{-} \rightarrow \gamma^{*} / Z_{0}^{*}$, Nucl. Phys. B 956 (2020) 115055 [arXiv:2003.14289] [INSPIRE].

[82] T. Huber and D. Maître, HypExp: A Mathematica package for expanding hypergeometric functions around integer-valued parameters, Comput. Phys. Commun. 175 (2006) 122 [hep-ph/0507094] [INSPIRE].

[83] K.S. Kolbig, J.A. Mignoco and E. Remiddi, On Nielsen's Generalized Polylogarithms And Their Numerical Calculation, CERN-DD-CO-69-5 (1969).

[84] K.S. Kolbig, Nielsen's generalized polylogarithms, SIAM J. Math. Anal. 17 (1986) 1232 [INSPIRE].

[85] A. Erdelyi, Higher Transcendental Functions, McGraw-Hill Book Company, Inc. (1953).

[86] G.F. Sterman, An Introduction to quantum field theory, Cambridge University Press (1993) [DOI].

[87] A.B. Goncharov, Multiple polylogarithms and mixed Tate motives, math/0103059 [INSPIRE].

[88] H. Frellesvig, D. Tommasini and C. Wever, On the reduction of generalized polylogarithms to $L i_{n}$ and $L i_{2,2}$ and on the evaluation thereof, JHEP 03 (2016) 189 [arXiv:1601.02649] [INSPIRE].

[89] C. Duhr and F. Dulat, PolyLogTools - polylogs for the masses, JHEP 08 (2019) 135 [arXiv: 1904.07279] [INSPIRE].

[90] C. Duhr, F. Dulat and B. Mistlberger, Drell-Yan Cross Section to Third Order in the Strong Coupling Constant, Phys. Rev. Lett. 125 (2020) 172001 [arXiv:2001.07717] [INSPIRE].

[91] C. Duhr, F. Dulat and B. Mistlberger, Charged current Drell-Yan production at $N^{3} L O$, JHEP 11 (2020) 143 [arXiv:2007.13313] [INSPIRE]. 